Aus der Klinik für Gastroenterologie und Endokrinologie (Prof. Dr. med. V. Ellenrieder) im Zentrum Innere Medizin der Medizinischen Fakultät der Universität Göttingen

\title{
Indikationen, Ergebnisse und klinischer Nutzen von 203 Dünndarmkapselendoskopien am Universitätsklinikum Göttingen
}

\author{
INAUGURAL - DISSERTATION \\ zur Erlangung des Doktorgrades \\ der Medizinischen Fakultät \\ der Georg-August-Universität zu Göttingen
}

vorgelegt von

Juliane Flemming

aus Berlin 
Dekan: Prof. Dr. rer. nat. H. K. Kroemer

I. Berichterstatter: Prof. Dr. med. T. Sauerbruch

II. Berichterstatterin: Prof. Dr. med. A. Müller-Dornieden

Tag der mündlichen Prüfung: 11. Februar 2015 
Ich möchte mich bei allen Personen bedanken, die mich bei der Erstellung dieser Arbeit unterstützt und motiviert haben sowie mir mit Rat und Tat zur Seite standen.

Mein größter Dank gilt meiner Betreuerin Silke Cameron. Sie hat mich während der gesamten Promotionsphase begleitet. Ohne sie wäre das Gelingen dieser Arbeit nicht möglich gewesen. Ihre Anregungen, Ideen und Kommentare haben maßgeblich zu dem Gelingen dieser Arbeit beigetragen. Vielen herzlichen Dank.

Weiterhin danke ich Herrn Prof. Dr. T. Sauerbruch dafür, dass er meine Doktorarbeit übernommen und in kurzer Zeit Korrektur gelesen hat. 


\section{Inhaltsverzeichnis}

\section{Abkürzungsverzeichnis}

1 Einleitung ........................................................................................................................ 1

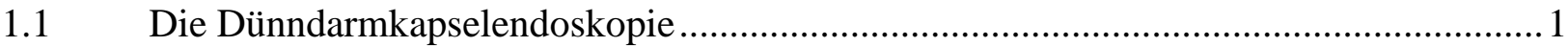

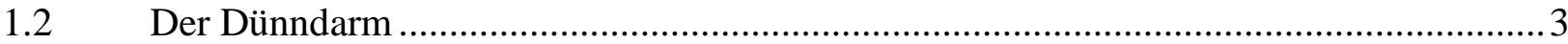

1.2.1 Durchblutungsstörungen und vaskuläre Dünndarmerkrankungen ..........................5

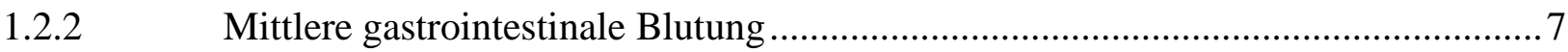

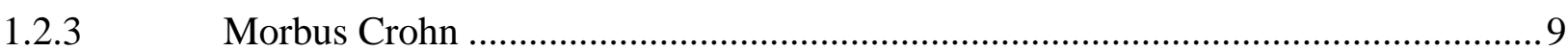

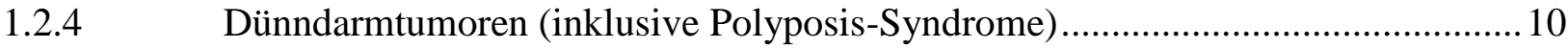

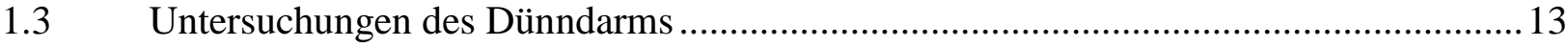

1.3.1 Anamnese, klinische Untersuchung und Differentialdiagnostik ......................... 13

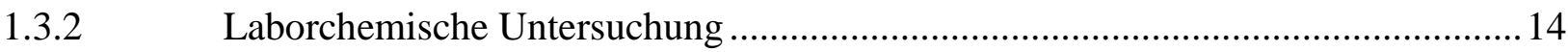

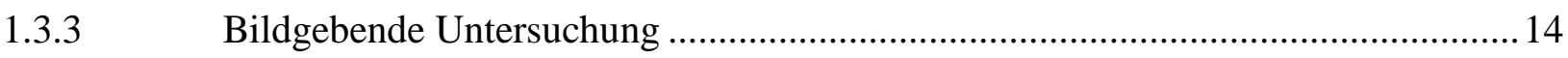

1.3.3.1 Ösophagogastroduodenoskopie …............................................................ 14

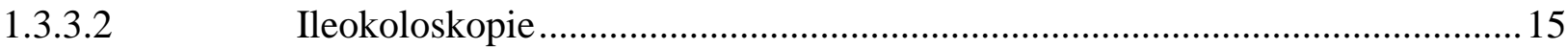

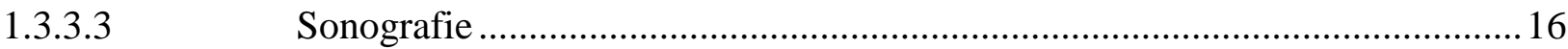

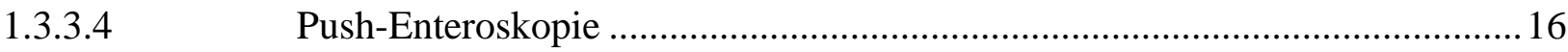

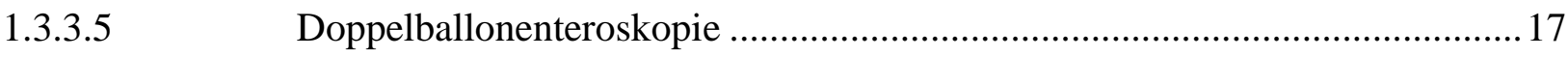

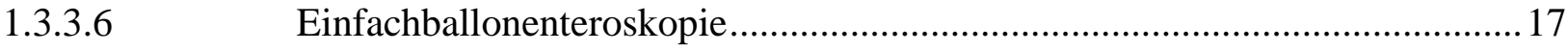

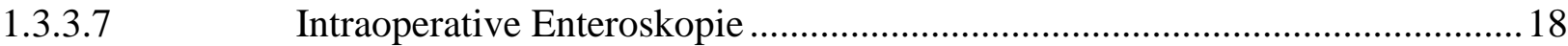

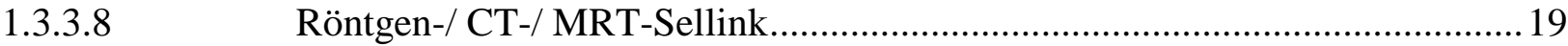

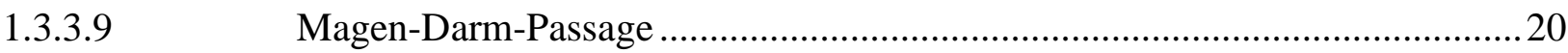

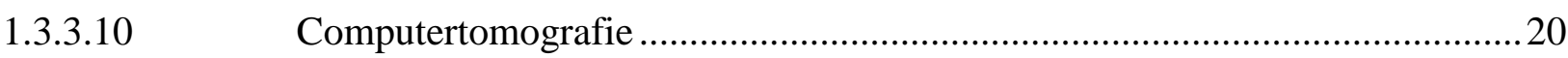

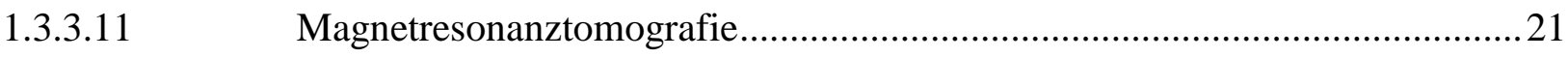

1.3.3.12 Positronen-Emissions-Tomografie/ PET-CT ..............................................22

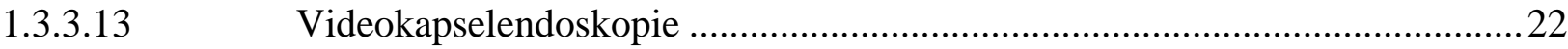

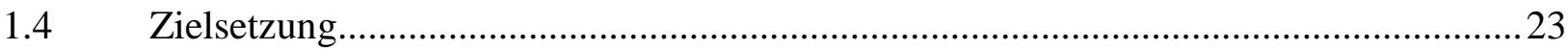


2 Material und Methodik...............................................................................224

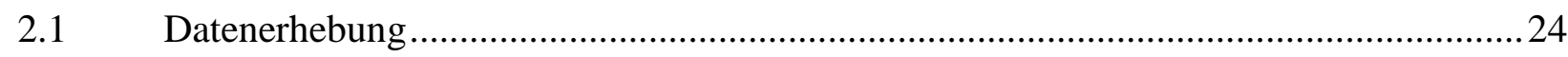

$2.2 \quad$ Einführung in die Videokapselendoskopie ........................................................... 24

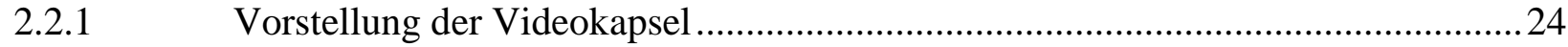

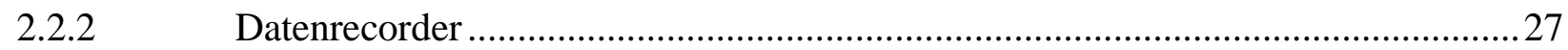

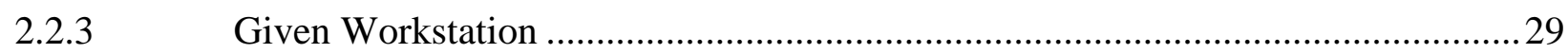

2.3 Indikationen, Kontraindikationen, Komplikationen .............................................. 31

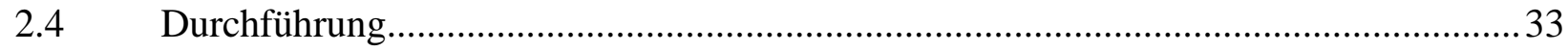

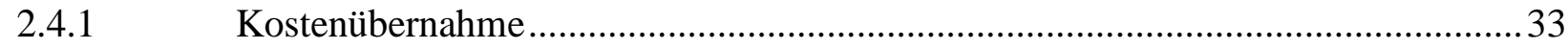

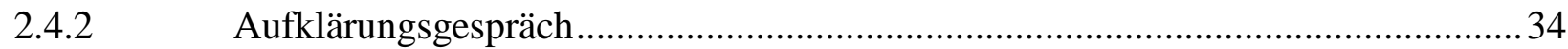

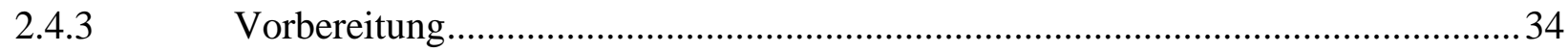

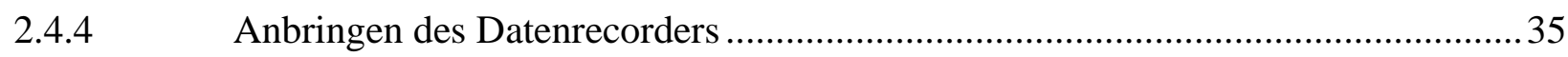

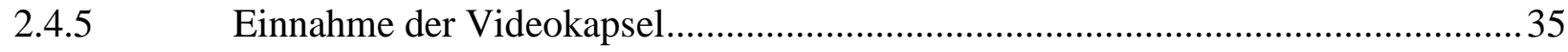

2.4.6 Auswertung der Untersuchungsergebnisse …................................................... 35

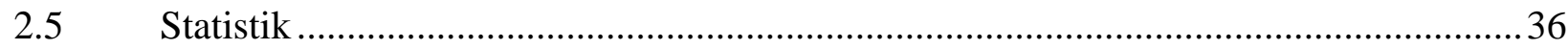

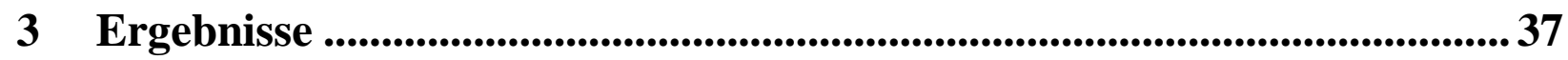

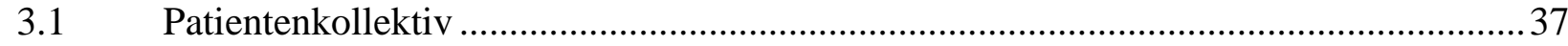

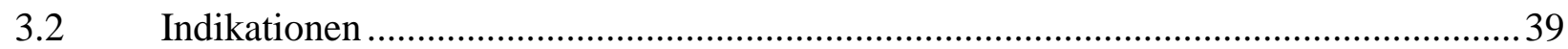

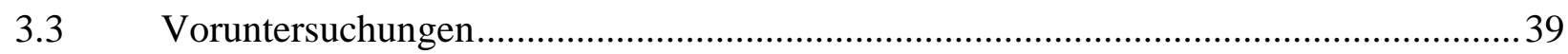

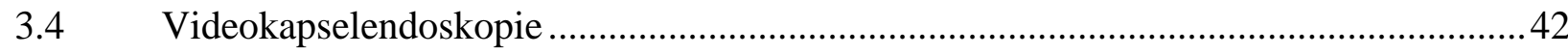

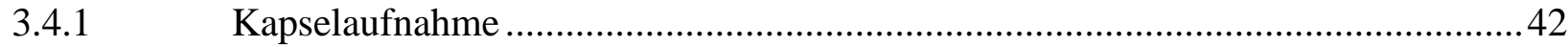

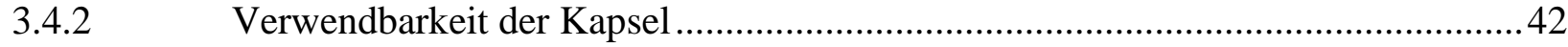

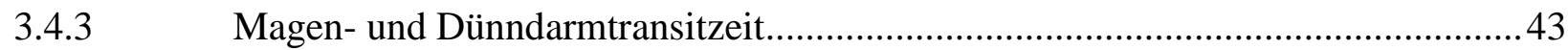

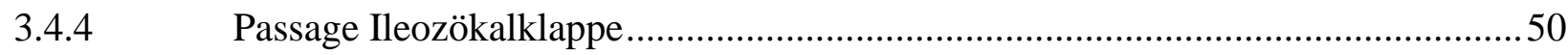

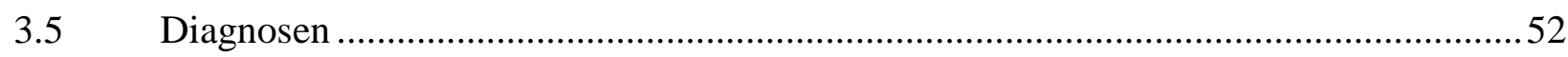

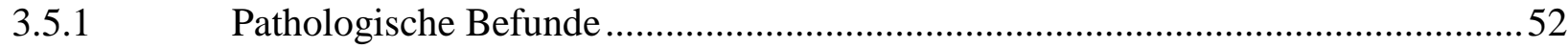

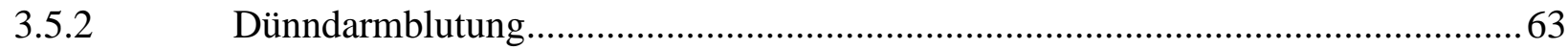

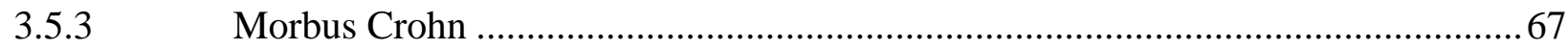

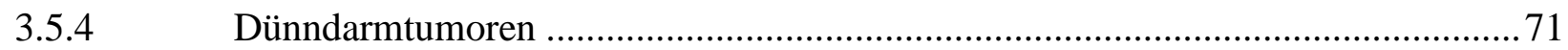

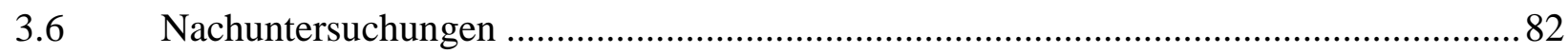




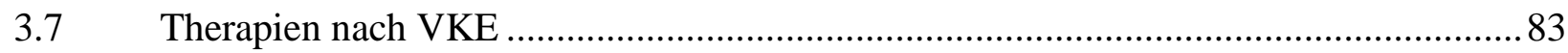

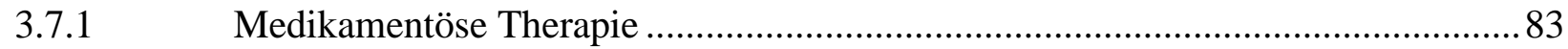

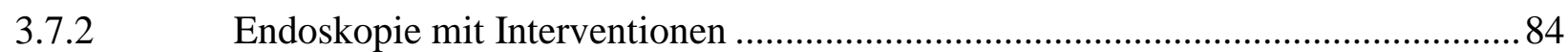

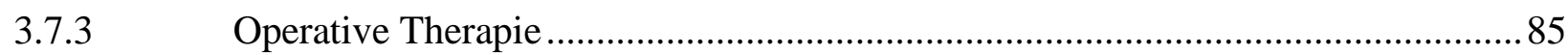

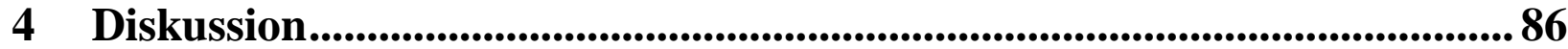

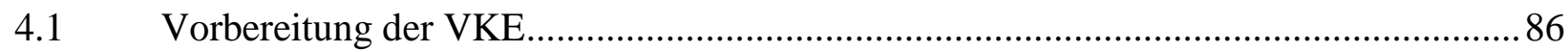

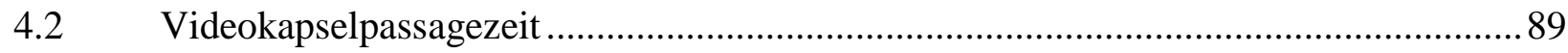

4.2.1 Videokapselpassagezeit bezüglich Alter und BMI .......................................... 89

4.2.2 Videokapselpassagezeit bei Diabetes mellitus-Patienten..................................... 89

4.2.3 Videokapselpassagezeit und Komorbidität ....................................................90

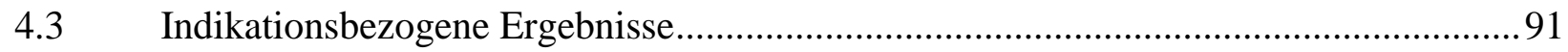

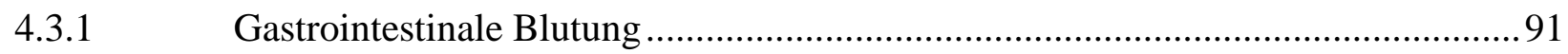

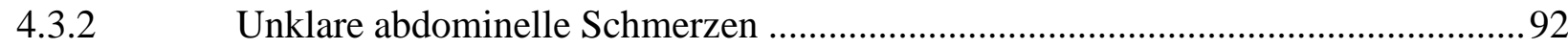

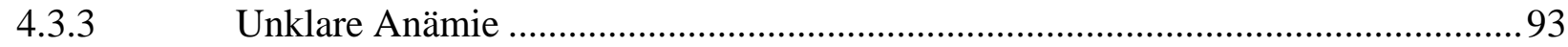

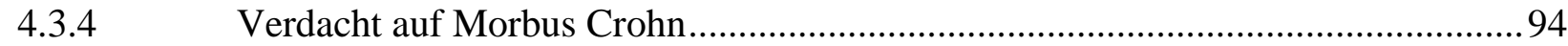

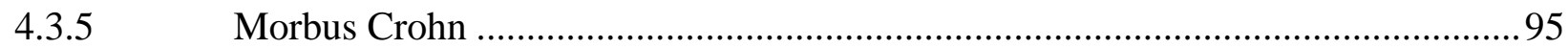

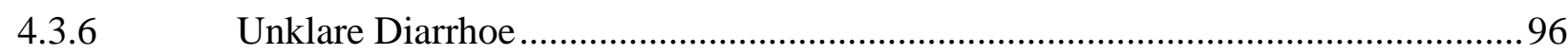

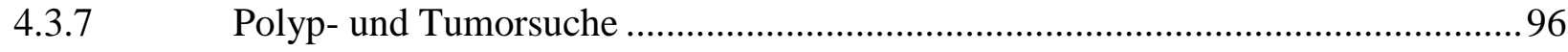

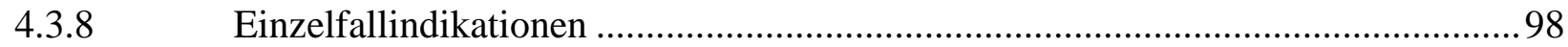

4.4 Komplikationen der Dünndarmkapselendoskopie..................................................99

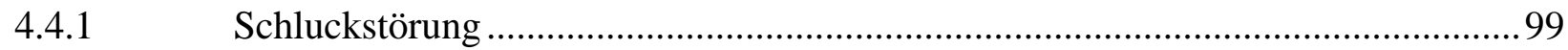

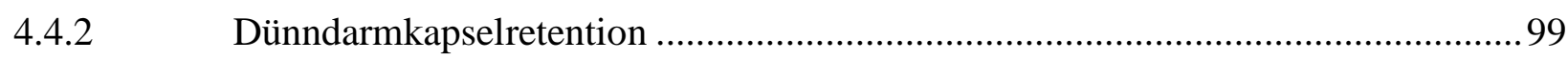

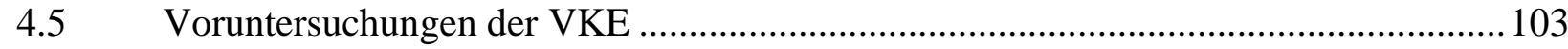

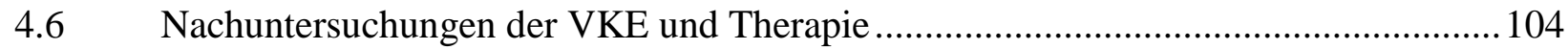

4.7 Tabellarischer Vergleich der verschiedenen Studien ............................................ 105

4.8 Vergleich der Kapselendoskopie mit anderen Untersuchungsmethoden .................... 108

4.8.1 Vergleich mit der Push-Enteroskopie .............................................................. 108

4.8.2 Vergleich mit der Doppelballonenteroskopie ................................................ 109

4.8.3 Vergleich mit Enteroklysma-Untersuchungen .................................................. 110

4.8.4 Vergleich mit der Angiografie/ CT-Abdomen .................................................. 111

4.8.5 Vergleich mit der Magen-Darm-Passage ........................................................... 111

4.8.6 Vergleich mit der intraoperativen Enteroskopie ............................................. 112

4.9 Ausblick: Zukunft der Dünndarmvideokapselendoskopie ..................................... 113 


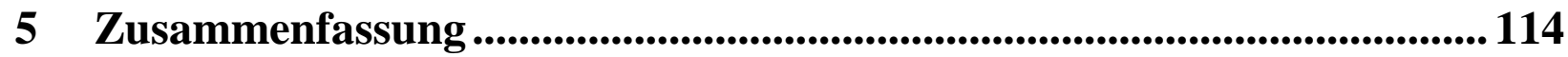

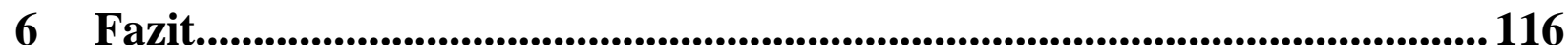

7 Abbildungsverzeichnis ................................................................................................... 118

8 Tabellenverzeichnis ................................................................................. 120

9 Literaturverzeichnis ........................................................................... 121 


\section{Abkürzungsverzeichnis}

\begin{tabular}{|c|c|}
\hline Abkürzung & Bedeutung \\
\hline Abb. & Abbildung \\
\hline APC & Argon-Plasma-Koagulation \\
\hline APUD & amine precursor uptake and decarboxylation \\
\hline bds. & beidseits \\
\hline BMI & Body-Mass-Index \\
\hline CA $19-9$ & Carbohydrate-Antigen 19-9 \\
\hline CART15 & caspase recruitment domain family, member 15 \\
\hline CCD & charge-coupled device \\
\hline $\mathrm{CE}$ & certified for Europe \\
\hline CEA & karzinoembryonales Antigen \\
\hline CMOS & complementary metal oxide semiconductor \\
\hline $\mathrm{CT}$ & Computertomografie \\
\hline DD & Differentialdiagnose \\
\hline DGVS & Deutsche Gesellschaft für Verdauungs- und Stoffwechselkrankheiten \\
\hline D.m. & Diabetes mellitus \\
\hline DTIC & Dacarbazin \\
\hline EK & Erythrozytenkonzentrate \\
\hline EKG & Elektrokardiogramm \\
\hline ERCP & endoskopisch retrograde Cholangiopankreatikografie \\
\hline FAP & familiäre adenomatöse Polyposis \\
\hline FDA & Food and Drug Administration \\
\hline FDG-PET & Fluorodeoxyglukose-Positronen-Emissions-Tomografie \\
\hline FICE & flexible intelligent colour enhancement \\
\hline GB & Gigabyte \\
\hline GI & gastrointestinal \\
\hline GIST & gastrointestinaler Stromatumor \\
\hline Gy & Gray \\
\hline
\end{tabular}




\begin{tabular}{|c|c|}
\hline h & Stunde \\
\hline $\mathrm{Hb}$ & Hämoglobin \\
\hline $\mathrm{HNO}$ & Hals-Nasen-Ohren \\
\hline $\mathrm{HPF}$ & high power field \\
\hline ICCE & International Conference of Capsule Endoscopy \\
\hline $\operatorname{Ig}$ & Immunglobulin \\
\hline J. & Jahre \\
\hline k.A. & keine Angaben \\
\hline KI & Konfidenzintervall \\
\hline LED & light-emitting diode \\
\hline LK & Lymphknoten \\
\hline M. & Morbus \\
\hline MCP & Metoclopramid \\
\hline MDT & Magen-Darm-Trakt \\
\hline $\mathrm{MHz}$ & Megahertz \\
\hline Min. & Minuten \\
\hline MRT & Magnetresonanztomografie \\
\hline M2A & mouth to anus \\
\hline $\mathrm{n}$ & number \\
\hline ND & Nebendiagnose \\
\hline NHL & Non-Hodgkin-Lymphom \\
\hline NOD & nucleotide-binding oligomerization domain \\
\hline NPV & negativer prädiktiver Wert (negative predictive value) \\
\hline NSAR & nicht steroidale Antirheumatika \\
\hline ÖGD & Ösophagogastroduodenoskopie \\
\hline $\mathrm{OP}$ & Operation \\
\hline p-ANCA & perinukleäre Autoantikörper gegen Granulozytenplasma \\
\hline PPV & positiver prädikiver Wert (positive predictive value) \\
\hline PTT & partielle Thromboplastinzeit \\
\hline py & pack years \\
\hline
\end{tabular}


R0-Resektion Entfernung des Tumors im Gesunden

RF Raumforderung

SB small bowel

SD standard deviation

u.a. unter anderem

UMG Universitätsmedizin Göttingen

V.a. Verdacht auf

vgl. vergleiche

VKE Videokapselendoskopie

vs. versus

$\overline{\mathrm{x}} \quad$ arithmetischer Mittelwert

Z.n. Zustand nach

z.T. zum Teil 


\section{Einleitung}

\subsection{Die Dünndarmkapselendoskopie}

Lange Zeit galt der Dünndarm zwischen Treitz'schem Band und terminalem Ileum als terra incognita der Gastroenterologie. Mit der Kapselendoskopie steht eine neue diagnostische Methode zur Verfügung. Das Prinzip wurde noch bis vor 15 Jahren als science fictionFantasie angesehen (Heuss 2004). Mittlerweile ist die Kapseluntersuchung des Dünndarms besonders in der Diagnostik der obskuren mittleren gastrointestinalen Blutung als neuester Stand der Technik etabliert (Barth et al. 2007).

Bereits 1981 wurden Ideen entwickelt, wie zukünftig der bis dahin nicht einsehbare Dünndarm visualisiert werden könnte. Der Ingenieur Dr. Gavriel Iddan vom israelischen Rüstungsunternehmen RAFAEL Advanced Defense Systems Ltd. wurde auf einer Tagung in Boston von dem befreundeten Gastroenterologen Professor Eitan Scapa an die Umsetzung dieser Idee herangeführt (Iddan und Swain 2004). Die Verwendung einer CCD-Chip-Kamera (charge-coupled-device) ermöglichte den Ingenieuren, die bis dahin benutzten flexiblen Endoskope kleiner und besser steuerbar zu machen (Iddan und Swain 2004). Der CCD-Sensor ist ein elektronisches Bauteil zum Transport elektrischer Ladungen, mit dem sich Bilder digital speichern lassen (Litwiller 2001).

Dr. Iddan entwickelte die Idee, den Kopf des Endoskops, auf dem sich der CCD-Chip befand, abzuschneiden, um einen kleinen Transmitter mit diesem zu verbinden und ihn dann frei ohne eine physikalische Verbindung bewegen zu lassen. Diese Idee war jedoch zunächst nicht umsetzbar, da die Chip-Kamera nach dem damaligen Stand der Technik lediglich zehn Minuten mit Miniaturbatterien funktionierte (Iddan und Swain 2004). Zudem wiesen CCDExperten darauf hin, dass es im Dünndarm zu wenige pathologische Veränderungen gebe, für die es sich lohne, eine non-invasive Untersuchungsmethode zu entwerfen. 1993 konnte ein Prototyp für biologische Experimente eingesetzt werden, welcher schon damals aus drei Komponenten bestand: der Kapsel mit Radiowellentransmitter, dem Recorder und der Workstation. Im Jahr 1994 wurde die Patentierung beantragt, die Suche nach Investoren für die weitere Entwicklung dieses Patentes gestaltete sich anfangs allerdings schwierig (Iddan und Swain 2004). 
In Kooperation mit Gavriel Meron, Manager einer Firma, die CCD-Kameras für medizinische Geräte einsetzte, gelang es Iddan, diesen Entwurf zu vervollständigen. Ab 1996 konnten die ersten tierexperimentellen Versuche an Schweinen durchgeführt werden (Iddan und Swain 2004).

1997 wurde das Patent für die verbesserte In-Vivo-Videokapsel erteilt, welche 2D-Bilder ermöglichte. Ein Jahr später gründete Meron die Firma Given Imaging®, welche den Vertrieb von medizinischen Technologien, insbesondere der Videokapselendoskope übernahm (Meron 2002). Bis heute ist diese Firma der Marktführer im Bereich Dünndarmvideokapseln. Seit 2005 führten drei weitere Firmen Videokapselsysteme ein:

1. OMOM Capsule ${ }^{\circledR}$ der Firma Jinshan Science and Technology (2005), Chongqing - China

2. MiRo-Cam ${ }^{\circledR}$ von Intromedic (2007), Seoul - Korea

3. EndoCapsule ${ }^{\circledR}$ der Firma Olympus (2008), Tokio - Japan (Steinbrück et al. 2010). Zur gleichen Zeit begann eine britische Forschungsgruppe, sich der Visualisierung des Dünndarms zu widmen. 1997 lernten sich der aus London stammende Gastroenterologe Professor Paul Swain und Meron auf einem gastroenterologischen Kongress kennen. Bereits ein Jahr später schloss sich Swain dem Team von Given Imaging® an. Beide Forscherteams profitierten von der Zusammenarbeit, sodass immer häufiger tierische und klinische Experimente durchgeführt werden konnten. Mit dem komplikationslosen Selbstversuch Swains, der 1999 die Kapsel schluckte, begann somit das Zeitalter der Videokapselendoskopie (Iddan und Swain 2004).

Die erste Publikation über die gemeinsame Erfindung und Entwicklung wurde im Januar 2000 im Fachmagazin Nature veröffentlicht (Iddan et al. 2000). Im August 2001 erhielt die Videokapsel namens M2A (mouth to anus) die amerikanische FDA-Zulassung sowie die europäische CE-Kennzeichnung (Meron 2002). Bereits Ende 2004 waren weltweit mehr als 120.000 Kapselendoskopien durchgeführt worden (Heuss 2004), im Jahr 2009 mehr als eine Million (Güldütuna und Keuchel 2009, S. 16).

2007 wurde die Videokapsel M2A, die später in PillCam SB (small bowel) umbenannt wurde, durch die innovative PillCam SB 2 ersetzt (Mishkin 2010).

In dieser Arbeit werden die ersten 203 Videokapselendoskopien analysiert, die im Zeitraum vom 25.01.2006 bis zum 16.11.2010 am Universitätsklinikum Göttingen konsekutiv durchgeführt wurden. 


\subsection{Der Dünndarm}

Der ca. 3 - 5 Meter lange Dünndarm (Intestinum tenue) dient hauptsächlich der Resorption von Nährstoffen mit vorausgehender Digestion und besitzt den größten Anteil des Wasserreservoirs des menschlichen Körpers (Kirsch 2011).

Der Dünndarm wird in drei Abschnitte untergliedert. Der erste Abschnitt, das Duodenum, welches unmittelbar hinter dem Pylorus beginnt, ist 25-30 cm lang, c-förmig und liegt (mit Ausnahme des Anfangs- und Endteils) vorwiegend retroperitoneal. Das Duodenum besteht aus vier Teilen: die Pars superior mit Bulbus duodeni - intraperitoneal gelegen - geht an der Flexura duodeni superior in die Pars descendens über, in welche die Papilla duodeni major (Vateri) mündet. Die Pars horizontalis schließt sich ab der Flexura duodeni inferior an und geht in die Pars ascendens - intraperitoneal gelegen - über (Kirsch 2011).

Der zweite Abschnitt des Dünndarms, das Jejunum, beginnt an der Flexura duodenojejunalis, welche das Ende des oberen Gastrointestinaltraktes darstellt, in Höhe des Treitz'schen Bandes (Kirsch 2011). Das Jejunum umfasst etwa $40 \%$ des oberen Dünndarmkonvolutes. Der Übergang zum letzten Abschnitt des Dünndarms, dem Ileum, ist fließend. Beide Darmabschnitte (Jejunum und Ileum) liegen intraperitoneal und sind am Mesenterium fixiert. Das Ileum macht etwa $60 \%$ des Dünndarmkonvolutes aus und endet an der Ileozökalklappe (Bauhin'sche Klappe) mit der Einmündung in das Kolon (Kirsch 2011). Der letzte Teil des Ileums, auch als terminales Ileum bezeichnet, kann ileokoloskopisch durch die Bauhin'sche Klappe erreicht und untersucht werden (Henniges et al. 2011, S. 18 f).

Die aus den Villi intestinales (Darmzotten) kommenden Lymphkapillaren vereinigen sich zu Lymphgefäßen über regionäre Lymphknoten in die Nodi mesenterici superiores (Lymphabfluss des Jejunums und Ileums) bzw. Nodi lymphoidei coeliaci (Lymphabfluss des Duodenums). Die Lymphe wird schließlich über den Truncus intestinalis in die Cisterna chyli weitergeleitet und gelangt über den Ductus thoracicus in den venösen Kreislauf. Die unterschiedlich großen Lymphknoten des Dünndarms stellen mit ca. 100-150 Lymphknoten die größte Lymphknotengruppe des menschlichen Körpers dar (Schünke et al. 2005, S. 300).

Die makroskopische Auffälligkeit des Dünndarms besteht aus den Plicae circulares (Kerckring-Falten), die aboral kontinuierlich an Höhe verlieren und in breiteren Abständen verlaufen, bis sie im terminalen Ileum nur noch vereinzelt zu finden sind. Die Lymphfollikel sind im letzten Teil des Dünndarms aggregiert und erscheinen als Peyer-Plaques (Folliculi lymphatici aggregati) (Lüllmann-Rauch 2009, S. 386). 
Die Plicae circulares entstehen durch Auffaltungen der inneren Darmwandschichten: Tunica mucosa und der Tela submucosa. Die äußeren Wandschichten bestehen aus der Tunica muscularis und der Serosa (Lüllmann-Rauch 2009, S. 380).

Wichtig für die Resorption von Nährstoffen ist die Oberflächenvergrößerung des Dünndarms (ca. $160 \mathrm{~m}^{2}$ ), welche durch die Mikrovilli bewirkt wird, die sich auf den Villi intestinales (Dünndarmzotten) befinden (Fritsch und Kühnel 2009).

Das Dünndarmepithel besteht aus einschichtigem Zylinderepithel, die Zotten und Krypten weisen unterschiedliche Zelltypen auf. An den Zotten sind Enterozyten (mit Mikrovilli an der apikalen Membran) und schleimbildende Becherzellen vorherrschend.

Charakteristisch für die Krypten sind:

- phagozytierende Paneth-Zellen mit antimikrobiellen Peptiden

- intestinale Stammzellen (Enterozyten), die eine begrenzte Lebensdauer von nur fünf bis sieben Tagen haben, an deren Ende sie apikal ins Darmlumen sezerniert werden

- enteroendokrine Zellen, die u.a. Gastrin, Sekretin, Cholezystokinin und Serotonin sezernieren (Lüllmann-Rauch 2009, S. 385).

Die Hauptaufgabe des Dünndarms ist die Resorption von Nahrungsbestandteilen, die durch die Verdauungsenzyme des Pankreas und die in der Leber produzierte Gallenflüssigkeit in chemische Bestandteile zerlegt werden. Die Resorption der Gallenflüssigkeit und des Vitamin B12 erfolgt im terminalen Ileum (Kirsch 2011).

Die Videokapselendoskopie (VKE) ermöglicht eine detaillierte, vergrößerte Darstellung der Dünndarmschleimhaut einschließlich Mukosa und deren Villi (Maßstab 1:8) (Keuchel und Hagenmüller 2005, S. 2). Wesentliche Charakteristika des normalen Dünndarms in der VKE sind aus der Tabelle 1 ersichtlich. 
Tabelle 1: Typische nicht pathologische VKE-Dünndarmkriterien (Keuchel et al. 2005, S. 36 f)

- gelborangefarbene Mukosa

- Villi intestinales

- Plicae circulares

- Papilla Vateri im Duodenum descendens

- kleine Gefäße, gelegentlich größere Venen

- Kontraktionen: propulsive und retropulsive Peristaltik

- Galle, Luftbläschen, Detritus im Sekret

- Lymphfollikel im terminalen Ileum

Im folgenden Kapitel werden die Erkrankungen des Dünndarms erläutert, die bei den Göttinger Patienten zur Videokapselendoskopie geführt haben.

\subsubsection{Durchblutungsstörungen und vaskuläre Dünndarmerkrankungen}

Im Unterschied zu akuten und chronischen Gefäßerkrankungen des Herzens, Gehirns oder der Extremitäten treten Durchblutungsstörungen des Darmmesenteriums relativ selten auf. Ursächlich hierfür ist die Kollateralisierung der drei Mesenterialarterien (Arteria mesenterica superior, Arteria mesenterica inferior, Truncus coeliacus) miteinander. Die Arteria mesenterica superior und die Arteria mesenterica inferior sind durch die Riolan-Arkade miteinander verbunden. Die Pankreas-Arkade verbindet den Truncus coeliacus mit der Arteria mesenterica superior (Hölscher und Hölscher 2006; Schünke et al. 2005, S. 266 f).

Akute mesenteriale Durchblutungsstörungen des Darmes können lebensbedrohlich sein, da der Dünndarm eine kurze Ischämietoleranzgrenze von 120-180 Minuten hat (Frieling 2009).

Der Mesenterialinfarkt beruht u.a. auf einer Arteriosklerose der Mesenterialarterien mit kombinierter arterieller Thrombose, einer arteriellen Embolie oder einer Venenthrombose. Diese akuten Durchblutungsstörungen gehen mit starken Schmerzen Angina abdominalis und häufig blutiger Diarrhoe einher (Herold 2012, S. 791).

Die chronischen Durchblutungsstörungen des Dünndarms können lange unerkannt bleiben, sie führen zu rezidivierenden, vor allem postprandialen Bauchschmerzen, kombiniert mit Gewichtsverlust. Die häufigste Ursache ist die Arteriosklerose im Zusammenhang mit Hyper- 
tonie, Diabetes mellitus, Adipositas, Hyperlipidämie sowie Zigaretten-Abusus. Endoskopisch kann man eine livide Schleimhaut oder Schleimhautläsionen sehen (Herold 2012, S. 791).

Weitere vaskuläre Dünndarmerkrankungen basieren auf arteriovenösen Gefäßveränderungen, zu denen beispielsweise die Angiektasien sowie die Venektasien zählen. Als Angiektasien werden Angiodysplasien und Teleangiektasien bezeichnet, die durch vaskuläre Malformation bzw. Dilatation der kapillären Blutgefäße in der Mukosa entstehen (Keuchel et al. 2005, S. 50). Diese Veränderungen können im gesamten Gastrointestinaltrakt auftreten (Meier und Manns 2000). Die genetische Variante der Angiektasien wird als hereditäre hämorrhagische Teleangiektasie, Morbus Osler-Rendu-Weber, bezeichnet. Zu den Symptomen zählen die vier Curaçao-Kriterien: Epistaxis, Teleangiektasien, viszerale Gefäßläsionen und die positive Familienanamnese (Keuchel et al. 2005, S. 50). Meist sind die Gefäßektasien kleiner als $5 \mathrm{~mm}$, diese Tatsache erschwert die Diagnostik und Detektion der Lokalisation akuter Blutungen (Braden 2003).

Man bezeichnet den venösen Anteil der vaskulären Dünndarmerkrankungen als Venektasien. Dabei handelt es sich um dauerhaft diffuse Erweiterungen oder Aussackungen einer Vene. Kleine Venektasien bluten in der Regel nicht, Varizen oder größere Venektasien können jedoch eine Blutungsgefahr darstellen (Keuchel et al. 2005, S. 54).

$\mathrm{Zu}$ den Dünndarmerkrankungen zählen weiterhin die intestinalen Lymphangiektasien. Kapselendoskopisch sind diese als weiße, teilweise geschwollene Dünndarm-Villi mit vereinzelten winzigen weißen Punkten zu erkennen, bei zystischen Lymphangiektasien bis hin zu korallenartigem Aussehen (Asakura et al. 1981). Die Weißfärbung beruht auf unzureichendem Abtransport von Chylomikronen in den dilatierten Lymphkapillaren (Keuchel und Hagenmüller 2005, S. 59). Die Lymphangiektasien können diffus, fokal oder fleckförmig auftreten. Funktionelle Lymphangiektasien besitzen im Gegensatz zu sekundären Lymphangiektasien bei intestinalen und extraintestinalen Grunderkrankungen mit Lymphabflussstörung (Morbus Crohn, intraabdominelle Tumoren wie beispielsweise Lymphome, Infektionen, z.B. Morbus Whipple mit dem Erreger Tropheryma whipplei, die Strahlenenteritis sowie die Rechtsherzinsuffizienz oder eine Ischämie) keinen Krankheitswert (Keuchel und Hagenmüller 2005, S. 59). Eine andere Erkrankung, bei der es zur Ausprägung einer Lymphangiektasie kommt, ist die primäre intestinale Lymphangiektasie. Diese sehr seltene angeborene Lymphgefäßmissbildung betrifft vorwiegend Kinder und Jugendliche. Durch Platzen von Lymphbahnen gelangen hierbei Lipide in die Faeces. Klinisch äußert sich 
diese Erkrankung in Diarrhoen und exsudativer Enteropathie mit Malassimilation und Eiweißverlust sowie Proteinmangelödemen (Keuchel und Hagenmüller 2005, S. 61).

\subsubsection{Mittlere gastrointestinale Blutung}

Die gastrointestinalen (GI-) Blutungen werden nach ihrer Lokalisation eingeteilt. Die obere gastrointestinale Blutung hat ihren Ursprung proximal des duodenojejunalen Übergangs. Die mittlere gastrointestinale Blutung entsteht zwischen dem Treitz'schen Band und der Ileozökalklappe und die untere gastrointestinale Blutung ist von dieser Klappe bis zum Analkanal definiert (Gölder und Messmann 2011).

Zwischen 3-5\% der mittleren gastrointestinalen Blutungen sind als obskur oder nicht diagnostizierbar klassifiziert (Lewis 2000). Die Amerikanische Gesellschaft für Gastroenterologie (AGA) bezeichnet eine obskure Blutung als eine Blutung unklarer Lokalisation, die weder in einer Gastroskopie noch in einer Koloskopie detektiert werden kann. Die okkulte chronische Blutung wird durch eine Eisenmangelanämie offensichtlich oder kann im Screening mit einem positiven Hämoccult-Test festgestellt werden. Eine akute Blutung äußert sich hingegen overt mit rezidivierenden sichtbaren Blutverlusten oral/ anal (Hämatemesis, Melaena, Hämatochezie) und führt oft zur Transfusionspflichtigkeit und/ oder Kreislaufdysregulation bis hin zum Volumenmangelschock (Scharinger et al. 2007).

Als Normwert des Universitätsklinikums Göttingen wurde der Hämoglobingehalt bei Frauen zwischen 11,5-15 g/dl und bei Männern zwischen 13,5 und 17,5 g/dl vorausgesetzt.

Als Ursachen von Blutungsquellen im Dünndarm stehen die Angiodysplasien mit ca. $40 \%$ an erster Stelle, gefolgt von medikamentös-induzierten Schleimhautläsionen wie Ulzera oder Erosionen mit ca. 15\%, z.B. durch nichtsteroidale Antirheumatika (NSAR). Seltener sind in ca. 5\% Grund einer gastrointestinalen Blutung: die primären Neoplasien und Metastasen sowie zu 2\% die M. Crohn-Ulzerationen des Dünndarms, die Polyposis-Syndrome, die Meckel-Divertikel oder die portal hypertensive Enteropathie (Braden 2003; Scharinger et al. 2007).

Erosionen sind Läsionen, die ausschließlich auf die Mukosa beschränkt sind und nicht die Muscularis mucosae überschreiten. Ulzera hingegen überschreiten die Muscularis mucosae. Diese Läsionen reichen bis in die Submukosa (Lüllmann-Rauch 2009, S. 378). Bei Nachweis von Ulzera wird zur Diagnosesicherung eine endoskopische Biopsie empfohlen. 
Es gibt weitere Faktoren, die eine gastrointestinale Blutung hervorrufen können: Herzkreislauf- und/ oder Lebererkrankungen, Nierenversagen mit Dialysepflichtigkeit sowie die Einnahme von Antikoagulanzien (Henniges et al. 2011, S. 165; Keuchel et al. 2005, S. 51). Bei einer dualen Hemmung der Thrombozytenaggregation mit ASS und Clopidogrel ist das Risiko für eine gastrointestinale Blutung um den Faktor vier erhöht (Hallas et al. 2006).

Auch das Alter des Patienten ist entscheidend für die Häufung von Rezidivblutungen sowie das Letalitätsrisiko (Gölder und Messmann 2011). Die häufigsten Ursachen obskurer gastrointestinaler Blutungen abhängig vom Alter sind in Tabelle 2 aufgelistet.

Tabelle 2: Altersabhängige Ursachen mittlerer gastrointestinaler Blutungen

(Scharinger et al. 2007)

\section{Patienten unter 40 Jahre}

1. Dünndarmtumoren

2. Morbus Crohn

3. Meckel-Divertikel

4. Polyposis-Syndrome

5. Angiodysplasien
Patienten über 40 Jahre

1. Angiodysplasien

2. Dünndarmtumoren

3. medikamentös induzierte Läsionen der Dünndarmschleimhaut

4. Dieulafoy-Läsionen

Die Kapselendoskopie ermöglicht nicht nur die Diagnosestellung der Blutung, sie kann auch das Blutungspotential abschätzen sowie die Lokalisation mit Hilfe der speziellen Software für eine Intervention bestimmen (Cave 2004).

Bei Vorliegen einer Gefäßmalformation bedarf es einer endoskopischen Intervention mit einer mono- oder bipolaren Elektrokoagulation, einer Argon-Plasma-Koagulation oder einer Lasertherapie (Braden 2003). Bei einer Läsion, die dem distalen Duodenum oder proximalen Jejunum zuzuordnen ist, kann die orale Anwendung eines pädiatrischen Koloskops oder PushEnteroskops hilfreich sein. Bei einer weiter distal gelegenen Läsion wird in der Literatur die Doppelballonenteroskopie empfohlen (May et al. 2010). Wenn diese Methoden erfolglos bleiben oder die Läsion zu groß ist, wird eine Operation - meist eine Dünndarmsegmentresektion - erforderlich (Keroack et al. 2004). Symptomatisch kann der Blutverlust mit kristalloiden oder kollidalen Infusionen, durch Eisensubstitution, Erythrozytentransfusionen und/ oder dem Pausieren von Thrombozytenaggregationshemmern bzw. oralen Antikoagu- 
lanzien therapiert werden (Keuchel et al. 2005, S. 51; Lewis 1999). Als Ultima Ratio kann die medikamentöse, jedoch nicht zugelassene Therapieoption der Antiangiogenesehemmer Bevacizumab (Avastin ${ }^{\circledR}$ ) oder Thalidomid in Rücksprache mit den Krankenkassen verabreicht werden (Lupu et al. 2013; Sami et al. 2014).

\subsubsection{Morbus Crohn}

Morbus Crohn ist eine chronische Erkrankung des Gastrointestinaltraktes, die zwei Häufigkeitsgipfel aufweist. Einerseits sind vorwiegend junge Erwachsene von 20 bis 30 Jahren betroffen, andererseits Menschen zwischen 60 und 70 Jahren (Böcker et al. 2008, S. 303).

In Deutschland liegt die Inzidenz bei 5,2 pro 100.000 Einwohner (Timmer und Goebell 1999). Die Entzündung ist charakterisiert durch eine diskontinuierliche und segmentale, chronisch granulomatöse Manifestation, die den gesamten Magen-Darm-Trakt, von der Mundhöhle bis zum After, mit mukosalem oder transmuralem Befall betreffen kann (Herold 2012, S. 469 f). Die häufigste Lokalisation des M. Crohn befindet sich mit 70\% im Dünndarm, wobei der Schleimhautbereich bei $40 \%$ der M. Crohn-Patienten im unteren Dünndarmabschnitt - dem terminalen Ileum - verändert ist. In 25\% der Fälle ist der M. Crohn im Kolon lokalisiert, zu 5\% sind der Magen und/ oder der Ösophagus betroffen (Voderholzer et al. 2006).

Typische Symptome sind kolikartige Schmerzen, vorwiegend im rechten Unterbauch, kombiniert mit unblutiger chronischer Diarrhoe (Herold 2012, S. 469 f). Die Erkrankung kann mit einer leichten Temperaturerhöhung und Gewichtsverlust einhergehen. Bei schwerer Beeinträchtigung kann es zu einer Malabsorption, einer Fistelbildung, Abszessen oder einer Darmstenose kommen, die zu dem klinischen Bild eines akuten Abdomens durch einen (Sub-) Ileus oder der Komplikation einer Perforation führen kann (Herold 2012, S. 469 f).

$\mathrm{Zu}$ der Diagnostik des Morbus Crohn tragen mehrere Untersuchungen bei: die Anamnese, das klinische Erscheinungsbild sowie eine Kombination aus biochemischen, sonografischen, endoskopischen, histologischen und/ oder radiologischen Befunden (Herold 2012, S. 469 f). Im Labor können folgende Veränderungen vorliegen: Erhöhung der Entzündungsparameter wie BSG und CRP, Leukozytose oder eine Anämie bedingt durch einen Blutverlust mit Eisenmangel oder einen Vitamin B12-Mangel (Herold 2012, S. 469 f).

Sonografisch werden bei Morbus Crohn-Patienten wandverdickte Darmabschnitte sowie eine vermehrte Durchblutung der Mesenterialgefäße und der Darmwand beschrieben. Zusätzlich 
können Fisteln, vergrößerte Lymphknoten oder freie Flüssigkeit detektiert werden. Bei Stenosen werden prästenotische Dilatationen auffällig (Delorme und Debus 2005).

Endoskopisch sind im Frühstadium fleckige Rötungen und aphthöse Schleimhautläsionen sichtbar. Im Laufe eines aktiven Schubs sind längs verlaufende fissurale Ulzerationen und ein Pflastersteinrelief typisch. Das Spätstadium manifestiert sich in Stenosen, Fisteln oder Abszessen (Melle et al. 2011). Anhand der Klinik und mit Hilfe von Stufenbiospien aus dem oberen Gastrointestinaltrakt, dem terminalen Ileum sowie aus dem Kolon kann die Diagnose M. Crohn gestellt werden (Melle et al. 2011). Histologisch findet man zu 40\% Epitheloidzellgranulome sowie skip lesions, ein diskontinuerliches lymphozytäres Infiltrat, ein Schleimhautödem und einen nicht erhaltenden Epithelzellverband (Riede und Blum 2009).

In der radiologischen Schnittbilddiagnostik der Computertomografie oder Magnetresonanztomografie mit Kontrastmittel nach Sellink gelten verdickte Dünndarmschlingen, ein ausgeprägtes Pflastersteinrelief, Abszesse, Fisteln und/ oder segmentale Stenosen als charakteristisch (Grützner und Schmid-Tannwald 2011, S. 437 ff).

Insbesondere bei Verdacht auf M. Crohn sollte vor der Kapselendoskopie ein StenoseAusschluss mit einer Kontrastmitteldarstellung in Form eines Röntgen- oder Magnetresonanz (MR)-Sellinks erfolgen (Henniges und Rosien 2011). Obligat bleibt die Patientenaufklärung über eine mögliche Komplikation - die Retention der Kapsel - vor der elektiven Untersuchung.

Bei der Diagnosestellung ist es entscheidend, andere entzündliche, toxische, vaskuläre, maligne oder infektiöse Ätiologie einer Enteritis und/ oder Colitis auszuschließen (Baumgart 2009).

\subsubsection{Dünndarmtumoren (inklusive Polyposis-Syndrome)}

Die Häufigkeit von Tumoren zwischen dem Pylorus und der Ileozökalklappe ist sehr viel geringer als im übrigen Gastrointestinaltrakt: Nur 1-5\% der malignen Darmtumoren sind im Dünndarm lokalisiert (Südhoff und Schmiegel 2007). Zehnmal häufiger als Malignome sind gutartige Dünndarmtumoren wie Adenome, Leiomyome, Lipome, inflammatorische Polypen sowie ektopisches Magen- und Pankreas-Gewebe, selten Endometriosen (Keuchel et al. 2006).

Folgende Grunderkrankungen weisen eine erhöhte Inzidenz von malignen Dünndarmtumoren auf: die familiäre adenomatöse Polyposis (FAP) inklusive des Gardner-Syndroms - einer 
phänotypischen Variante der FAP-, das Peutz-Jeghers-Syndrom, das hereditäre nichtpolypöse Kolonkarzinom (HNPCC), die Neurofibromatose, die nichttropische Sprue sowie der M. Crohn mit einem Krankheitsverlauf von 20 Jahren (Herbay et al. 2004).

80\% der FAP-Patienten entwickeln Adenome im Dünndarm, das Lebenszeitrisiko für ein Dünndarmkarzinom wird auf 5-10\% geschätzt (Südhoff und Schmiegel 2007). Bei der Diagnose dieser genetischen Erkrankung ist eine endoskopische Seitblickoptik der Papillenregion sehr entscheidend, da nach Iaquintos Studie die Wahrscheinlichkeit von Tumoren im Jejunum und Ileum erhöht ist, wenn an der Papille im Duodenum auch Adenome vorhanden waren (Iaquinto et al. 2008). Deshalb ist die Kapselendoskopie bei FAP indiziert.

Zu den malignen Entitäten des Dünndarms zählen die Adenokarzinome (47\%), die gastroenteropankreatischen neuroendokrinen Tumoren (GEP-NET) (28\%), die Sarkome (12\%) und die Lymphome (12\%) (Lewis et al. 2006).

Das Ursprungsgewebe des Karzinoms sind Epithelien, die neuroendokrinen Karzinome (Karzinoide) gehen aus bestimmten hormonbildenden enterochromaffinen Zellen (APUD) hervor, das Sarkom aus Muskel- und Bindegewebe und das Lymphom entstammt dem Lymphgewebe (Böcker et al. 2008, S. 198).

Die seltenen, aber häufig im Dünndarm auftretenden mesenchymalen Tumoren sind die gastrointestinalen Stromatumoren (GIST). Sie entstehen aus den Cajal-Zellen der Intestinalwand oder ihren Vorläuferzellen. Ihre Inzidenzrate beträgt eine Neuerkrankung pro 100.000 Einwohner pro Jahr (Herold 2012, S. 467). Vorwiegend ist der GIST im Magen vertreten, jedoch ist die Hochrisikoform mit einem erhöhtem Rezidivrisiko und/ oder Metastasen vermehrt im Dünndarm vorhanden. Die Malignität hängt von der Tumorgröße, der intestinalen Lokalisation sowie der Mitoserate ab (Lewis et al. 2006).

Sekundäre maligne Dünndarmtumoren sind Metastasen vorwiegend von extraintestinalen Tumoren wie beim malignem Melanom, Magen- oder Pankreas-Karzinom, seltener bei Bronchial-, Mamma- und Ovarialkarzinomen (Washington und McDonagh 1995) sowie Nierenzellkarzinomen (Südhoff und Schmiegel 2007).

Dünndarmtumoren werden erst in einem fortgeschrittenen Stadium symptomatisch, wenn der Tumor eine gewisse Größe erreicht hat (Hauser 2006). Patienten leiden an abdominellen Schmerzen und Übelkeit, verursacht durch eine Obstruktion im Rahmen eines (Sub-) Ileus (Herold 2012, S. 467). Darüber hinaus können intestinale Blutungen auftreten, die zu einer Eisenmangelanämie führen (Albert et al. 2008). Die Blutungen treten häufiger bei den beni- 
gnen Tumoren auf, wohingegen sich die malignen Tumoren meist mit Obstruktionen manifestieren (Minardi et al. 1998). Weiterhin geben die Patienten vor der Diagnosesicherung tumorbegleitende Symptome wie Gewichtsverlust und Abgeschlagenheit an. Wenn Dünndarmmalignome lymphogen oder hämatogen metastasieren, können zusätzliche Beschwerden auftreten (Müller 2012/13, S. 190 f). 


\subsection{Untersuchungen des Dünndarms}

\subsubsection{Anamnese, klinische Untersuchung und Differentialdiagnostik}

Wichtige anamnestische Fragen, die jedem Patienten in der Gastroenterologie gestellt werden müssen, betreffen die Themen: Veränderung der körperlichen Leistungsfähigkeit, Fieber, Gewichtsverhalten, Nachtschweiß, das Erfragen von abdominellen Schmerzen sowie die Stuhlfarbe, Stuhlhäufigkeit und Stuhlbeschaffenheit (Riemann und Rosenbaum 2010).

Die klinische Untersuchung umfasst neben der Beurteilung des Allgemeinzustandes die Farbe und Beschaffenheit von Haut und Schleimhäuten, die Palpation und Auskultation des Abdomens, wie auch die digitale Untersuchung der Rektumampulle auf Blut, Stuhlkonsistenz, Sphinktertonus und auf eine mögliche Raumforderung (Riemann und Rosenbaum 2010).

Bei einer okkulten Blutung, die zu einer unklaren Eisenmangelanämie führen kann, sollte neben einer gastroenterologischen Abklärung in erster Linie an eine Abdomen-Sonografie, eine Gastroskopie und Koloskopie gedacht werden. Bei negativen Resultaten kann zusätzlich eine HNO-ärztliche, gynäkologische oder urologische Untersuchung durchgeführt werden bzw. der Ausschluss einer hämatologischen Systemerkrankung erfolgen (Riemann und Rosenbaum 2010).

Bei Patienten mit einer Eisenmangelanämie, bei denen keine gastrointestinalen Symptome vorliegen, kann eine Eisenresorptionsstörung, z.B. im Rahmen einer Zöliakie, die Ursache sein (Thiel et al. 2011). Patienten, die das Symptom Diarrhoe und/ oder übel riechenden Meteorismus aufweisen, sollten auf eine Laktoseintoleranz untersucht werden (Rosien et al. 2011, S. 23, 25).

Allgemein gehen Dünndarmerkrankungen meist mit chronischer wässriger Diarrhoe sowie voluminösen Fettstühlen bei Malabsorption einher (Thiel et al. 2011). Dickdarmerkrankungen wie die Colitis ulcerosa äußern sich beispielsweise mit unregelmäßigen Stühlen, chronischen Diarrhoen, analem Schleim- und Blutabgang (Rosien et al. 2011, S. 214). 


\subsubsection{Laborchemische Untersuchung}

Zur Abklärung einer okkulten Blutung rät die Literatur neben der Bestimmung eines Blutbildes und des Ferritinspiegels (Diagnostik der Eisenmangelanämie) zu einem Hämoccult-Test (Zuckerman et al. 2000).

Um eine chronisch entzündliche Darmerkrankung im aktiven Stadium, insbesondere den M. Crohn, zu diagnostizieren, sind das Blutbild und erhöhte Entzündungsparameter wie der CRP-Wert hilfreich. Auch ein Calprotectin-Nachweis im Stuhl sollte bestimmt werden, da dieser Test sensitiver als der CRP-Wert sein kann (Melle et al. 2011).

Für den Nachweis einer glutensensitiven Enteropathie besteht neben der tiefen Dünndarmbiopsie ein hochsensitiver Test auf Autoantikörper gegen IgA- bzw. IgG- Transglutaminase (Herold 2012, S. 463).

Die laborchemische Diagnostik bei Verdacht auf Malignität schließt einen Hämoccult-Test, das Blutbild, die Leberenzyme sowie den Tumormarker CEA mit ein (Müller 2012/13, S. 190 f). Wenn ein gastrointestinaler Stromazelltumor vermutet wird, lässt sich zu 90\% mit der Immunhistochemie die Expression von c-KIT nachweisen. Ein wichtiger Screeningparameter zum Ausschluss eines neuroendokrinen Tumors ist das Chromogranin A im Serum, das in $75-95 \%$ bei NET-Tumoren erhöht ist (Südhoff und Schmiegel 2007).

\subsubsection{Bildgebende Untersuchung}

\subsubsection{1 Ösophagogastroduodenoskopie}

Goldstandard für die Diagnostik von oberen gastrointestinalen Blutungen mit den Hauptsymptomen Hämatemesis oder Meläna ist die Ösophagogastroduodenoskopie (ÖGD), die gleichzeitig Gewebeentnahmen, Maßnahmen zur Blutstillung und/ oder Stenosebehandlung ermöglicht (Kohler et al. 2010, S. 191). Mit einem Gastroskop, welches an der Spitze mit einer Videokamera versehen ist, lassen sich der Ösophagus, der Magen und das Duodenum bis zum Treitz'schen Band beurteilen. Da es sich bei einer ÖGD um eine invasive Methode zur Diagnostik und Therapie handelt, ist eine Aufklärung über den genauen Ablauf der Untersuchung, die Indikation und Risiken sowie die Sedierungsart unerlässlich (Kohler et al. 2010, S. 187). Der Patient muss eine schriftliche Einverständniserklärung vorschriftsmäßig 
24 Stunden vor Untersuchungsbeginn ausfüllen und unterschreiben, Ausnahmen sind NotfallEndoskopien oder vitale Indikationen (Kohler et al. 2010, S. 193).

Vor der Untersuchung werden die Thrombozyten sowie der Quick-Wert bestimmt (Kohler et al. 2010, S. 193).

Bei einer oberen gastrointestinalen Blutung kann die Blutungsquelle in über $95 \%$ endoskopisch lokalisiert werden, in 15-30\% sind mehrere Blutungsquellen vorhanden (Gölder und Messmann 2011). Wenn eine Notfallendoskopie bei schwerer Gastrointestinalblutung erforderlich ist, muss der Patient zuerst kreislaufstabilisiert und ggf. zum Schutz vor einer Aspiration intubiert werden, ein Anheben der Gerinnungsparameter kann zusätzlich notwendig sein (Biecker et al. 2008; Sauerbruch 2008).

\subsubsection{Ileokoloskopie}

Die Ileokoloskopie ist eine Untersuchung des unteren Verdauungstraktes. Sie lässt eine Beurteilung des Kolorektums und des distalen Anteils des Dünndarms - des terminalen Ileums - sowie Biopsien und Interventionen zu (Henniges et al. 2011, S. 54). Da dieses Verfahren auch eine invasive Methode ist, muss der Patient vorher sein schriftliches Einverständnis abgeben. Eine Aufklärung über die Indikation, die Risiken, den Ablauf und die Art der Sedierung ist unumgänglich. Für eine gute Sicht im Kolon ist eine gründliche Darmreinigung durch eine orthograde Spülung erforderlich. Das Vorbereitungsregime an der UMG beginnt am Vortag der Untersuchung mit dem Trinken von jeweils zwei bis drei Litern Polyethylenglykol- (PEG-) Lösung: Bei der Gabe von Moviprep® werden zwei Beutel dieser Sorte in zwei Liter lauwarmem Wasser aufgelöst und getrunken, zusätzlich werden weitere drei bis vier Liter Wasser getrunken. Ambulante Patienten erhalten ggf. zusätzlich am Morgen des Untersuchungstages noch einen Liter PEG-Lösung zum Trinken. Bei Patienten, die nicht selbst ausreichend trinken können, kann die Reinigungslösung über eine Magensonde verabreicht werden. Ferner erhalten die Patienten am Vortag und ggf. am Tag der Untersuchung jeweils ein salinisches Klistier à $100 \mathrm{ml}$ (Yal® in der UMG) zur rektalen Anwendung. Absolute Kontraindikationen sind ein klinischer Verdacht auf Perforation, HerzKreislauf-Schock oder instabile Kreislaufverhältnisse. Als relative Kontraindikationen gelten ein hochakuter Schub einer chronisch-entzündlichen-Darmerkrankung, eine hochakute Colitis mit Läsionen und vulnerabler Wandung sowie pathologische Gerinnungsparameter (Henniges et al. 2011, S. 54). 


\subsubsection{Sonografie}

Die Sonografie ist eine nicht-invasive, wenig belastende Untersuchung, die in der Gastroenterologie eine zentrale Rolle für die Beurteilung von Stadium und Aktivität manifester Erkrankungen des Bauchraumes und der angrenzenden Nachbarregionen einnimmt. Die Bildgebung des Abdomens mit Schallwellen umfasst die Bauchorgane, den Darm, die Pleura, die großen abdominellen Gefäße und die ableitenden Harnwege. Als Echtzeitverfahren besitzt sie eine hohe räumliche Auflösung (Pachmann et al. 2011).

\subsubsection{Push-Enteroskopie}

Die Push-Enteroskopie steht für eine partielle endoskopische Untersuchung des Dünndarms. Das Enteroskop ist mit $2 \mathrm{~m}$ länger als das Gastroskop $(1 \mathrm{~m})$ oder das Koloskop (1,30 bis 1,60 m). Diese Untersuchung wurde in den 1990er Jahren eingeführt. Mit der Push-Enteroskopie ist der Dünndarm von oral bis zum Treitz'schen Band und von anal bis zum terminalen Ileum einsehbar. Das gesamte Ileum lässt sich aus diesem Grund nicht beurteilen (Kohler et al. 2010, S. 240 f).

Der Patient erhält in der Regel für den Zeitraum der Untersuchung eine intravenöse Sedierung oder eine allgemeine Anästhesie (Braden 2003). Die Detektionsrate von Blutungsquellen liegt bei 21-38\% (Henniges et al. 2011, S. 55). Die Diagnostik und eine ggf. interventionelle Therapie erfolgen beim Geräterückzug (Kohler et al. 2010, S. 240 f). Um die Sicht auf die Schleimhaut zu verbessern, können bei der Push-Enteroskopie eine Luftinsufflation sowie ein Vor- und Zurückschieben des Endoskops vorgenommen werden. Jedoch können Läsionen hinter Schleimhautvorwölbungen übersehen werden (Saurin et al. 2003). 


\subsubsection{Doppelballonenteroskopie}

Die Doppelballonenteroskopie ist eine Push-and-Pull-Enteroskopie (PPE). Mit der seit ca. 2003 in Deutschland eingeführten Methode kann bei erfahrenen Untersuchern der komplette Dünndarm von oral und/ oder anal endoskopisch eingesehen werden. Gleichzeitig können auch therapeutische Maßnahmen durchgeführt werden (Henniges et al. 2011, S. 55). Die PPE ist eine Ergänzung zur VKE. Die diagnostische Ausbeute liegt weltweit bei 70-80\% (Henniges et al. 2011, S. 55).

Das System der PPE setzt sich zusammen aus einem $200 \mathrm{~cm}$ langen hochauflösenden VideoEndoskop/ Enteroskop mit einem Außendurchmesser von 8,5 mm und einem $140 \mathrm{~cm}$ langen Übertubus mit einem Außendurchmesser von 12 mm (Hartmann 2010). An den Enden des Enteroskops sowie des Übertubus befinden sich mit Luft aufblasbare Latexballone, die ein Auffädeln des Dünndarms und somit die Diagnostik ermöglichen (Kohler et al. 2010, S. 243).

Die PPE wird unter stationären Krankenhausbedingungen durchgeführt, da sie in der Regel therapeutisch eingesetzt wird. Das Vorbereitungsprocedere gleicht der einer Koloskopie.

Bei mittleren, schweren und persistierenden GI-Blutungen wird die Doppelballonenteroskopie zur Blutstillung verwendet (Sugano und Marcon 2007). Die Blutstillung erfolgt durch Injektionen mit Adrenalin, seltener Fibrin oder Argon-Plasma-Koagulation bei Angiodysplasien. Ebenfalls ist die Applikation eines Hämastase-Clips möglich. Auch Polypektomien, Biopsien und Chromoendoskopie, Tusche- oder Clipmarkierung für eine elektive Operation können bei diesem Untersuchungsverfahren vorgenommen werden. Zudem sind Dilatationen von Stenosen wie auch Mukosaresektionen möglich (Kohler et al. 2010, S. 243).

Jedoch kann es durch den Übertubus zu Verletzungen in der Schleimhaut des Magens und des Duodenums bis hin zur Perforation kommen (Henniges et al. 2011, S. 57). In der Literatur wurde auch das Auftreten einer Pankreatitis durch die Verletzung an der Papille beschrieben (May 2007).

\subsubsection{Einfachballonenteroskopie}

Ein weiteres Verfahren zur endoskopischen Dünndarmdiagnostik ist die seit 2001 bestehende Single-Ballon-Enteroskopie. Diese Methode wird in der endoskopischen Abteilung der UMG verwendet. Das Enteroskop von Olympus besteht aus einem $200 \mathrm{~cm}$ langen Arbeitskanal mit einer flexiblen Spitze am distalen Ende sowie einem $132 \mathrm{~cm}$ langen Übertubus mit einem aufblasbaren Ballon (Hartmann et al. 2007). 
Das Enteroskop wird durch den Übertubus bis zu einer nicht überwindbaren Biegung, meist die Flexura duodenojejunalis am Treitz'schen Band, vorgeschoben und dann durch Abwinklung der Spitze („Einhaken") fixiert. Nach Aufblasen des Ballons wird das Gerät jeweils bis zur nächsten Darmabknickung vorge-schoben, wo sich die Spitze wieder einhakt. Der Teil, der sich von oral aus nicht einsehen lässt, ist in der Regel retrograd über das Kolon und das terminale Ileum erreichbar. Auf diese Weise lässt sich mit dem Enteroskop theoretisch der gesamte Dünndarm untersuchen (Hartmann und Riemann 2009).

\subsubsection{Intraoperative Enteroskopie}

Die intraoperative Enteroskopie wurde Mitte der 1980er Jahre als Untersuchungstechnik des Dünndarms etabliert. Bis zur Einführung der Kapselendoskopie im Jahr 2001 war diese Enteroskopie die einzige Methode den gesamten Dünndarm zu erreichen (Henniges et al. 2011, S. 55).

Durch eine mediane Laparotomie kann der mobilisierte Dünndarm mit Hilfe einer Enterotomie durch ein in der Mitte des Dünndarms eingesetztes Endoskop, in der Regel ein dünnkalibriges Koloskop, betrachtet werden (Hartmann und Riemann 2009). Zunächst wird der Dünndarm so aufgefädelt, dass beim Rückzug vom Treitz'schen Band oder von der Bauhin'schen Klappe die Schleimhaut betrachtet werden kann. Die endoskopische Variante lässt neben dem chirurgischen Eingriff alle Möglichkeiten der Diagnostik und Intervention zu (Hartmann und Riemann 2009). Sie erfordert eine gute Kooperation zwischen einem Chirurgen und einem Gastroenterologen. Der Chirurg assistiert dem Endoskopiker, indem er den Dünndarm auf das Endoskop auffädelt.

Heutzutage sollte eine Operation lediglich als Ultima Ratio vorgenommen werden, wenn alle Methoden endoskopisch wie auch radiologisch bei der Blutstillung versagen (Hartmann und Riemann 2009).

Nur bei intermittierenden Blutungen unklarer Lokalisation ist es angebracht, eine explorative Laparotomie durchzuführen. Besonders die Angiodysplasien können mit der intraoperativen Endoskopie gut lokalisiert werden (Gölder und Messmann 2011). Jedoch kann die explorative Laparotomie mit einer geringen Letalitätsrate von 0,01-0,03\% verbunden sein (Henning 2000). 


\subsubsection{Röntgen-/ CT-/ MRT-Sellink}

Seit der Einführung der Röntgen-Doppelkontrast-Untersuchung des Dünndarms im Jahre 1971, auch Enteroklysma nach Sellink genannt, war dies lange Zeit die einzige Möglichkeit, Dünndarmerkrankungen wie Fisteln, Stenosen oder Tumoren darzustellen (Grützner und Schmid-Tannwald 2011, S. 439 f). Schleimhautveränderungen wie eine flache Mukosa oder vaskuläre Läsionen sind mit den Enteroklysma-Verfahren nicht darstellbar (Scharinger et al. 2007). Blutungsquellen lassen sich nur selten detektieren (Sensivität 15\%) (Henniges et al. 2011, S. 55). Bei Patienten mit Verdacht auf eine Ileus- oder Hohlorganperforation ist bariumhaltiges Kontrastmittel kontraindiziert, stattdessen muss ein wasserlösliches Kontrastmittel (z.B. Gastrografin) verwendet werden (Grützner und Schmid-Tannwald 2011, S. 437). Nachteilig sind die hohen Kosten dieser Untersuchung. Entsprechende Patientenaufklärungen sind sowohl bei der Röntgendurchleuchtung als auch bei dem CT-/ MRT-Enteroklysma erforderlich.

Bei dem konventionellen Enteroklysma wird unter Röntgensicht eine spezielle Sonde mit der Spitze in Höhe des Treitz'schen Bandes platziert. Der Doppelkontrast entsteht durch die nacheinander folgende Verabreichung von verdünnter Bariumsulfatlösung, das sich an die Darmwände anlegt, zur Darstellung der Darmwände, und von Methylzellulose für die Ansicht des Darmlumens (Grützner und Schmid-Tannwald 2011, S. 437).

Aktuell wird in der Dünndarmdiagnostik das MRT-Enteroklysma zum Erkennen von Dünndarmtumoren, eines M. Crohn oder extraluminaler Prozesse eingesetzt. Dafür ist keine Strahlenexposition erforderlich. Das CT-Enteroklysma - eingesetzt in der Akutdiagnostik weist hingegen eine hohe Strahlenbelastung auf (Grützner und Schmid-Tannwald 2011, S. $439 \mathrm{f})$.

Für diese Untersuchungen ist eine 12-stündige Nahrungskarenz des Patienten erforderlich. Eine Darmreinigung mit einer hyperosmolaren Elektrolytlösung (ca. ein bis zwei Liter) wird zudem empfohlen (Grützner und Schmid-Tannwald 2011, S. 439).

Die Anwendung eines CT-Enteroklysma mit Kontrastmittelapplikation schreibt eine Bestimmung von TSH- und Kreatinin-Werten vor (Alkadhi 2011, S. 223, 229 f).

Etwa eine Stunde vor der Untersuchung trinkt der Patient eine intraluminale Kontrastmittellösung (z.B. Mannitol, Methylzellulose), eine Applikation über eine nasojejunale Sonde wie beim konventionellen Enteroklysma ist auch hier möglich. Während der Untersuchung erhält der Patient zur Verminderung der Eigenperistaltik des Darmes ein Spasmolytikum (Butylscopolaminbromid). Zusätzlich wird bei Fehlen von Kontraindikationen zur besseren 
Darmbeurteilung intravenös ein Kontrastmittel injiziert (CT: nichtionisch jodhaltig, MRT: Gadolinium) (Grützner und Schmid-Tannwald 2011, S. 440).

\subsubsection{Magen-Darm-Passage}

Die Magen-Darm-Passage ist ein Verfahren, welches heute nur noch selten zur Anwendung kommt. Das Kontrastmittel, in der Regel Gastrografin, wird oral verabreicht (Wismer 2011). Häufige Indikationen stellen die Suche nach einer hochgradigen Stenose oder einem Ileus im Magen-Darm-Trakt dar (Egger et al. 2012). Flache mukosale und subepitheliale Läsionen wie bei Angiodysplasien können nicht erkannt werden (Scharinger et al. 2007). Als positive Nebenwirkung ist bekannt, dass dieses Medikament stuhlabführend wirkt, so dass es bei Patienten mit Koprostase eine therapeutische Wirkung hat (Wismer 2011). Die Strahlenbelastung ist hierbei gering (ca. $7 \mathrm{mSv}$ ), da keine kontinuierliche Röntgendurchleuchtung des Abdomens wie beim CT-Abdomen (ca. $10 \mathrm{mSv}$ ) erfolgt (Egger et al. 2012). Stattdessen werden Röntgenaufnahmen lediglich in bestimmten Zeitabständen nach Kontrastmitteleinnahme erstellt. Da Gastrografin ein jodhaltiges Kontrastmittel ist, muss eine Hyperthyreose und/ oder eine Jodallergie vor der Applikation ausgeschlossen werden (Grützner und SchmidTannwald 2011, S. 439 f).

\subsubsection{Computertomografie}

Die Computertomografie (CT) ist ein Schnittbildverfahren, das 1971 zum ersten Mal angewandt wurde (Hünerbein 2011). Der größte Vorteil gegenüber den bis dahin bekannten Verfahren war der hohe Weichteilkontrast (Flohr 2011).

In den letzten Jahrzehnten wurde die Methode deutlich verbessert, so dass die CT heute ein etabliertes Verfahren darstellt. Dank der modernen Technik dauert heute eine Untersuchung des Abdomens nur noch wenige Minuten. Für eine optimale Darstellung des Magen-DarmTraktes erfolgt eine orale Kontrastierung mit einem jodhaltigen Kontrastmittel und einer intravenösen Kontrastmittelapplikation (Leschka und Glaser-Gallion 2011). Die Aufklärung des Patienten ist sowohl für die Röntgenstrahlenbelastung als auch für die Verabreichung von Kontrastmittel erforderlich und sollte wie üblich 24 Stunden vor Untersuchungsbeginn durchgeführt werden. Für diese Untersuchung müssen die TSH- und Kreatinin-Werte des Patienten vorliegen, welche im Normbereich liegen sollten (Alkadhi 2011, S. 223, 229 f). 


\subsubsection{Magnetresonanztomografie}

Die Magnetresonanztomografie (MRT) ist ein nicht-invasives bildgebendes Verfahren, welches 1973 zum ersten Mal eingesetzt wurde (Radeleff et al. 2011). Vom MRT geht keine ionisierende Strahlenexposition aus. Durch die Magnetfelder und Radiofrequenzwellen können die Weichteile des Körpers, insbesondere der Verdauungstrakt, angeregt und somit auf Schnittbildern sichtbar gemacht werden (Radeleff et al. 2011). Durch die unterschiedliche Gewichtung von Wasser und Gewebe ist für die Beurteilung des Dünndarms keine Kontrastmittelapplikation über eine duodenale Sonde notwendig, eine Ausnahme stellt das MRT-Enteroklysma dar (Grützner und Schmid-Tannwald 2011, S. 440). Eine bessere Darstellung von den Weichteilgeweben kann durch Anwendung von jodfreiem Kontrastmittel erreicht werden.

Diese Methode ermöglicht eine präzise Lokalisation bei folgenden Komplikationen von chronisch-entzündlichen Darmerkrankungen namentlich des M. Crohn: Abszesse, Fisteln, Stenosen oder Konglomerattumoren. Begrenzt nachweisbar sind diskrete aphthöse oder entzündliche Veränderungen in der Dünndarmschleimhaut (Hartmann 2005).

Jedoch überwiegen die Vorteile, so dass der Einsatz eines Magnetresonanztomogramms zur Beurteilung eines M. Crohn im Dünndarm als die Schnittbild-Methode der Wahl betrachtet werden kann (Albert 2010).

Kontraindikationen sind medizinische Implantate wie Herzschrittmacher oder andere metallische Implantate sowie Klaustrophobie (Radeleff et al. 2011).

Der Patient muss keine Nahrungskarenz einhalten, ein schriftliches Einverständnis in diese Untersuchung ist aber notwendig. Nachteilig sind die hohen Untersuchungskosten von ca. $460 €$ pro Abdomen-MRT in der UMG. 


\subsubsection{Positronen-Emissions-Tomografie/ PET-CT}

Die Positronen-Emissions-Tomografie (PET) ist ein bildgebendes Verfahren der Nuklearmedizin, welches ein Aktivitätsverteilungsmuster in Schnittebenen rekonstruiert (Bartenstein et al. 2011). Dafür wird häufig das radioaktiv wirksame Isotop ${ }^{18} \mathrm{~F}$ des Fluors in Form von Fluordesoxyglukose (18-FDG) verwendet, welches eine Halbwertzeit von über 100 Minuten aufweist (Bartenstein et al. 2011). Die Verteilung von 18-FDG im Körper, ermöglicht ein Auffinden von Tumoren und Metastasen durch den erhöhten pathologischen Stoffwechsel. Für eine exakte anatomische Zuordnung dieser Aktivität wird die PET mit der CT verbunden (Bartenstein et al. 2011).

\subsubsection{Videokapselendoskopie}

Wenn die endoskopische Standarddiagnostik mit Gastroskopie, Koloskopie und ggf. SellinkBildgebung keine Blutungsquelle feststellen konnte, ermöglicht die VKE eine Lokalisation in den Dünndarmabschnitten. Dies trifft insbesondere dann zu, wenn die Blutung fortbesteht oder okkult bleibt (Kohler et al. 2010, S. 244 f). Wegen der einfachen Durchführbarkeit und der nicht-invasiven Methode wird die Kapselendoskopie bei einer gleichen Diagnoserate (Sensitivität 95\%, Spezifität $75 \%$ ) im Vergleich zur intraoperativen Enteroskopie primär bei obskuren Blutungen angewendet (Hartmann et al. 2005). Bei der Erkrankung des Morbus Crohn lassen sich Schleimhautveränderungen des Dünndarms mit der VKE sehr gut erkennen. Sie kann die entzündlichen und aphthösen Prozesse gut detektieren (Güldutuna und Keuchel 2009, S. 17). 


\subsection{Zielsetzung}

In dieser Dissertation sollen folgende Fragen beantwortet werden:

1.) Welche Voruntersuchungen wurden vor der VKE beim Patientenkollektiv durchgeführt?

2.) Wie ist die Zusammensetzung des Patientenkollektivs in Bezug auf Alter, Geschlecht, BMI, stationäre oder ambulante Untersuchung?

3.) Welche Beschwerdesymptomatik/ Indikation veranlasste die Videokapselendoskopie?

4.) Mit welchen Methoden wurden die Videokapseln zugeführt?

5.) Wie viel Prozent der durchgeführten VKE waren auswertbar?

6.) Welche Magen- und Dünndarmtransitzeiten wiesen die Patienten auf?

7.) Ist die Kapselverweildauer abhängig vom Patientenalter, BMI, Erkrankungen des endokrinen Systems (Diabetes mellitus), der Bauchorgane und/ oder Magen-DarmTrakt-Operationen?

8.) Wie hoch ist die diagnostische Ausbeute in der Detektion pathologischer Befunde über die endoskopischen Standardverfahren hinaus? Bezugnehmend auf die:

1. Blutungs- und Anämie-Abklärung

2. Diagnosestellung Morbus Crohn

3. Detektion von Tumoren im mittleren Gastrointestinaltrakt.

9.) Wie häufig konnte die Ileozökalklappe passiert werden? Wie hoch ist die Komplikationsrate von Retention und/ oder Stenosen?

10.) Welche Nachuntersuchungen/ Therapien resultierten aus den VKE-Ergebnissen? 


\section{Material und Methodik}

\subsection{Datenerhebung}

Dieser retrospektiven Studie liegen Daten von 203 Patienten zugrunde, die im Zeitraum vom 25.01.2006 bis zum 16.11.2010 eine Videokapselendoskopie in der gastroenterologischen Abteilung des Universitätsklinikums Göttingen erhielten. Für die Untersuchungen wurden Videokapseln der Firma Given Imaging® verwendet: die PillCam SB® - früher M2A-Kapsel® (mouth to anus) - und die PillCam SB 2@.

\subsection{Einführung in die Videokapselendoskopie}

Für die Durchführung der Videokapselendoskopie sind drei Bestandteile des PillCam-Systems erforderlich: Die Kapsel selbst, ein Datenrecorder für die Speicherung der Bilder sowie eine Workstation (bestehend aus einem Computer mit einer speziellen Software) für die Auswertung der Bilder (Given Imaging 2008, S.2).

\subsubsection{Vorstellung der Videokapsel}

Abb. 1: PillCam SB 2® im Vergleich zu einer $1 €$-Münze

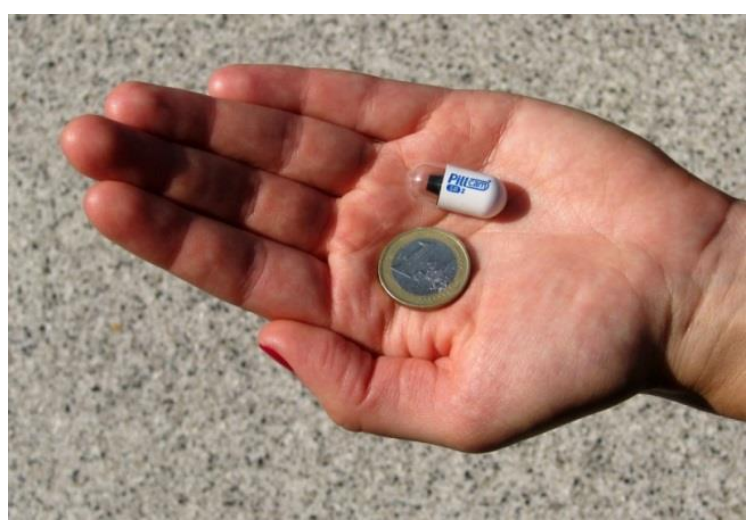

Die Videokapsel PillCam SB 2® (Abb. 1) besitzt die Form einer größeren Vitaminkapsel, sie ist $11 \times 26$ mm groß und wiegt 2,89 ( $\pm 0,15)$ g (Given Imaging 2008, S. 138). 
Das glatte Gehäuse aus Kunststoff, welches mit einem „biokompatiblen Überzug“ versehen ist, erleichtert die Ingestion und verhindert das Anhaften während der Passage durch den Verdauungstrakt oder eine Schädigung durch die im Magen-Darm-Trakt vorhandenen Verdauungssekrete (Güldütuna und Keuchel 2009, S. 12).

Die Kapsel selbst ist ein Einmalprodukt und wird anal via naturalis ausgeschieden (Güldütuna und Keuchel 2009, S. 12).

Die wichtigsten Eigenschaften dieser Kapsel, sind aus der Tabelle 3 zu entnehmen.

Tabelle 3: Eigenschaften der PillCam SB 2® (Given Imaging 2008, S. 138; Güldütuna und Keuchel 2009, S. 13)

\begin{tabular}{ll}
\hline Größe: & $11 \times 26 \mathrm{~mm}$ \\
Blickwinkel: & $156^{\circ}$ \\
effektive Sicht: & Entfernung: $3 \mathrm{~cm}$ \\
mindestens erkennbares Objekt: & unter $0,1 \mathrm{~mm}$ \\
Bildrate: & 2 oder 4 pro Sekunde \\
Batteriedauer: & 9 Stunden \\
Bilderfrequenz: & $50.000-65.000$ \\
Übertragung: & Radiofrequenz \\
chemische Sicherheit: & auflösungsresistent von $\mathrm{pH}=2-8$ \\
Betriebstemperatur: & $20-45^{\circ} \mathrm{C}$ \\
\hline
\end{tabular}

Abb. 2: Given Imaging® PillCam SB 2® (Zeichnung nach Güldütuna und Keuchel 2009, S. 13; mit freundlicher Genehmigung UNI-MED Verlag)

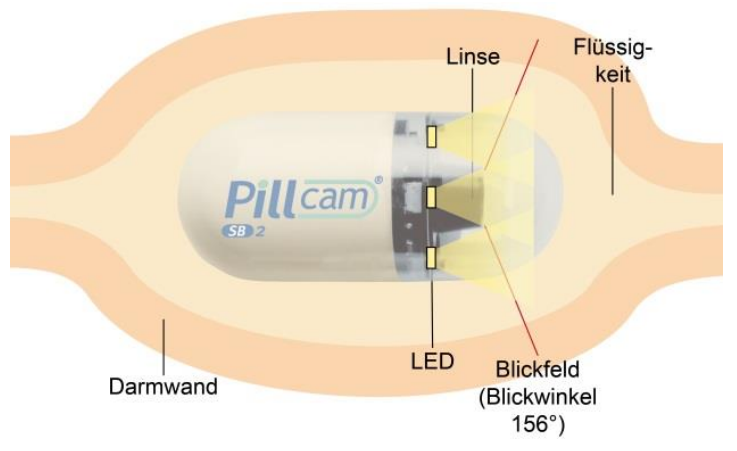

Lage der Videokapsel im Dünndarm

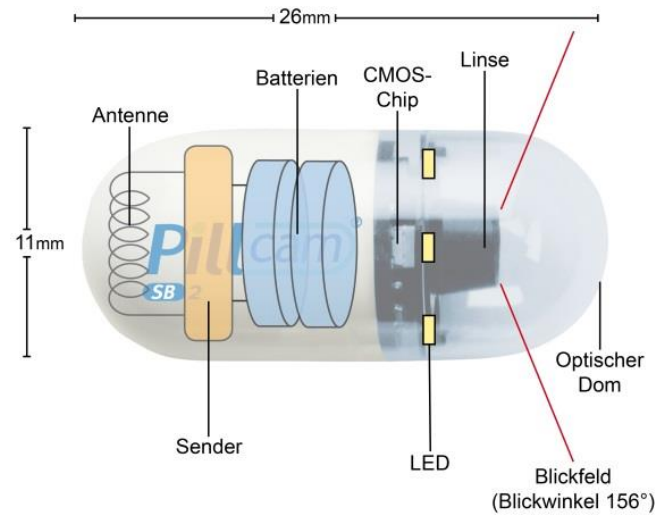

schematischer Aufbau der Videokapsel 
Aus der Abbildung 2 ist ersichtlich, dass eine Lichtquelle von vier weißen Leuchtdioden (LED) für die Illumination des dunklen Verdauungstraktes sorgt (Given Imaging 2008, S. 138). Durch ein transparentes optisches Fenster fällt das Licht durch drei Linsen und wird von einer CMOSChip-Kamera (complementary metal oxide semiconductor) in Bilder digitalisiert (Keuchel und Hagenmüller 2005, S. 2). Der Chip kann nur schwarz-weiße Bildinformationen aufnehmen. Über eine integrierte Software werden die ermittelten Grauwerte mit Hilfe der Bayer-Maske zu einem Farbbild (RGB = rot, grün, blau) hochgerechnet (Stich et al. 2009). Der CMOS-Chip lässt die Bilder in einer Pixelgröße von 256x256 entstehen. Die Auflösung der Kamera von 0,1 mm sorgt für eine Vergrößerung von 1:8 (Keuchel und Hagenmüller 2005, S. 2). Jede Sekunde werden zwei Bilder erzeugt, wobei der Verdauungstrakt durch die Lichtblitze der LEDs erhellt wird. Ein in den Chip integrierter Schaltkreis sendet Signale über eine Radiofrequenz mit $433 \mathrm{MHz}$ an einen sich in der Kapsel befindenden Radiosender (Keuchel und Hagenmüller 2005, S. 2). Der dafür benötigte Strom wird von zwei Silberoxid-Batterien mit einer Laufzeit von neun Stunden erzeugt. In dieser Zeit können bis zu 65.000 Bilder aufgezeichnet werden (Güldütuna und Keuchel 2009, S. 12). Der mit einer Antenne versehende Radiosender schickt die Signale an die Sensoren, welche direkt am Körper oder am Gürtel (SensorBelt) angebracht sind, und die Signale zu dem ebenfalls am Gürtel befindlichen Datenrecorder (DataRecorder) weiterleiten, wo diese schließlich gespeichert werden (Güldütuna und Keuchel 2009, S. 12). Durch die standardisierte Position der Elektroden kann die Software jedes Signal, welches von der Videokapsel gesendet wird, einer genauen Lokalisation zuordnen und diese später auf der Workstation grafisch darstellen (Güldütuna und Keuchel 2009, S. 12).

Im Zeitraum Januar 2006 - Beginn der regelmäßigen Anwendung an der UMG - bis Dezember 2007 wurde die PillCam SB® für die Videokapselendoskopien eingesetzt. Seit 2007 wird die verbesserte PillCam SB ${ }^{\circledR}$ verwendet, welche eine stärkere Lichtintensität (SB®: 1 Linse, SB 2®: 3 Linsen) sowie eine bessere bildliche Auflösung ermöglicht (Metzger et al. 2009). Darüber hinaus wurde der Blickwinkel von $140^{\circ}$ auf $156^{\circ}$ erweitert, wodurch die Sicht auf die Dünndarmschleimhaut optimiert wurde (Metzger et al. 2009). Auch konnte die Batterieleistung von acht auf neun Stunden erhöht werden (Steinbrück et al. 2010). Die diagnostische Ausbeute ist im Vergleich zu der älteren Kapsel PillCam M2A/SB® kaum verändert $(p>0,05)$. Jedoch zeigen die Bilder der neueren Kapsel PillCam SB $2 \circledR$ eine bessere Gesamterscheinung durch: verbesserte Bildschärfe und Auflösung sowie bessere Lichtverhältnisse (Metzger et al. 2009). 


\subsubsection{Datenrecorder}

Die von der Videokapsel produzierten Bilddaten werden auf dem DataRecorder 3 (Abb. 3 links) empfangen und gespeichert. Diesen Recorder tragen die Patienten mittels eines verstellbaren Tragegürtels (RecorderBelt) direkt am Körper oder in einer Umhängetasche (Abb. 3 rechts).

Abb. 3: Given Imaging® Datenrecorder Typ 3 (links: DataRecorder in der Ladestation)
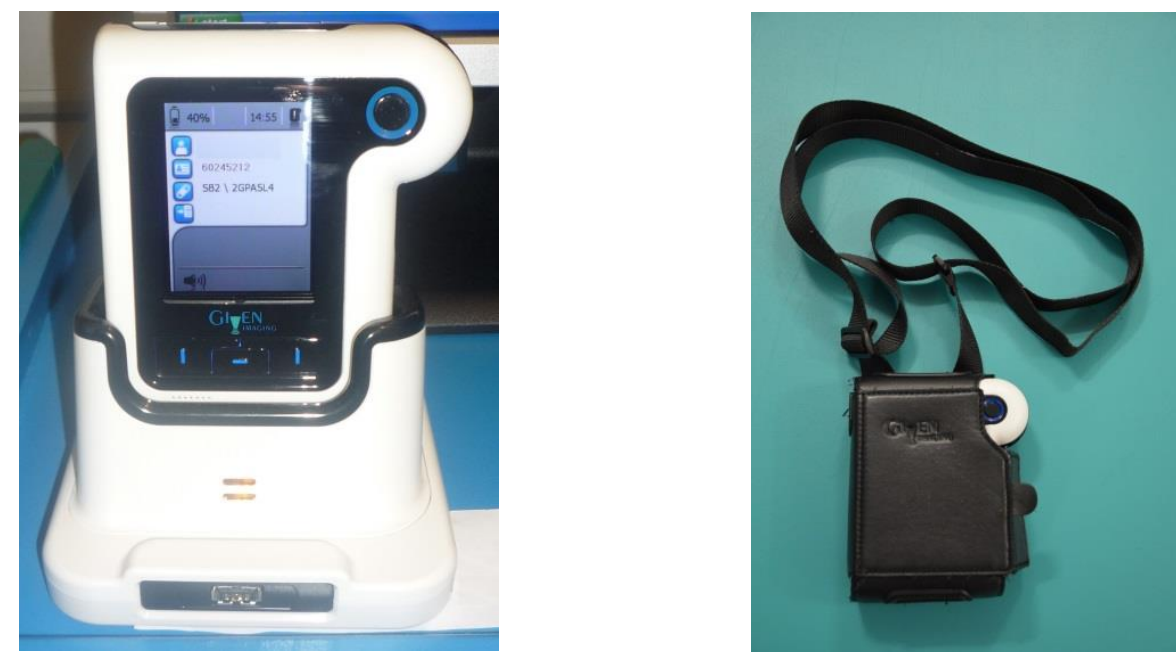

Der akkubetriebene Datarecorder sollte vollständig vor der Untersuchung in einer Basisstation aufgeladen werden (Given Imaging, S. 27). Er ist anwendungsbereit, wenn erstens die Elektroden (SensorArray) angeschlossen, zweitens die Patientendaten in der Workstation eingegeben und an den Recorder übermittelt wurden und drittens die Lithium-Ionen-Batterie aufgeladen ist (Given Imaging 2008, S. 4f, 45). Die Aufzeichnung im Magen-Darm-Trakt startet, sobald die Kapsel von dem Magneten in der Aufbewahrungsbox (Blister) getrennt wurde und ein Signal über das SensorArray an den Recorder sendet (Given Imaging 2008, S. 45). Die Datenübermittlung ist anhand eines Blinkens an der Kapsel-Leuchtanzeige am Recorder sichtbar. Ein Standby-Modus sorgt dafür, dass die Batterielaufzeit erst startet, wenn die Videokapsel Signale an den Recorder sendet und der Recorder sich außerhalb der Basisstation befindet. Nach 90 Minuten Standby-Zeit schaltet sich der Recorder automatisch ab (Given Imaging 2008, S. 5). Das SensorArray für die Dünndarmvideokapselendoskopie besteht aus acht aufklebbaren Elektroden - in der Größe für Kinder und Erwachsene variabel - und gleicht dem Aussehen eines Langzeit-EKG-Gerätes (Abb. 4). Jeder Sensor ist über ein flexibles Kabel mit dem DataRecorder verbunden. Die Kontaktierung des Sensors mit der Haut des Patienten erfolgt durch eine 
elastische Leiterplatte und eine Einweg-Haftmanschette (Given Imaging 2008, S. 9). Dafür müssen sich die Patienten vorher rasieren, da die Elektroden sonst nicht haften (Given Imaging 2008, S. 36). Alle Komponenten der PillCam-Plattform bestehen aus latexfreien Materialien (Given Imaging 2008, S. 9).

Seit 2010 wird in der UMG der SensorBelt eingesetzt, der das SensorArray ersetzt. Er wird wie ein Gürtel getragen, ist deshalb sehr komfortabel und erspart ein Anlegen der Elektroden.

\section{Abb. 4: Given Imaging ${ }^{\circledR}$ SensorArray}

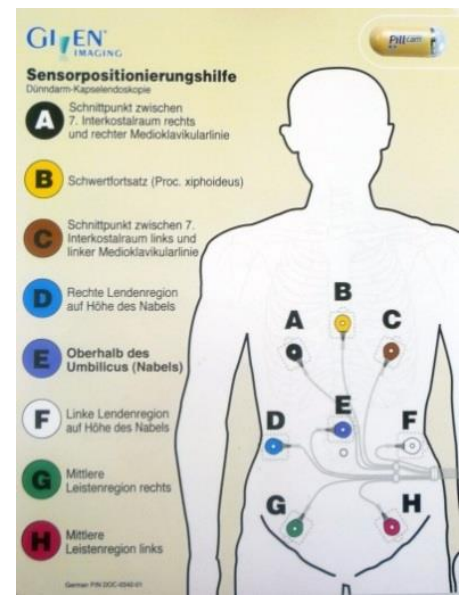

Positionierungspunkte der Sensoren (Given Imaging 2008, S. 38)

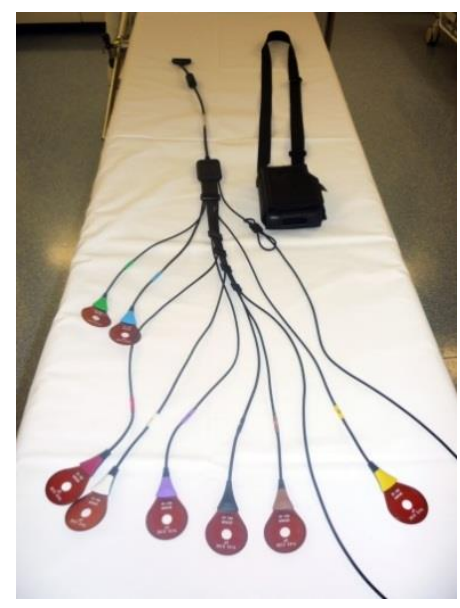

Elektroden mit Datenträgertasche 


\subsubsection{Given Workstation}

Nach erfolgter Speicherung der Dünndarmbilder auf dem DataRecorder werden die Bilder auf die Given Workstation (Abb. 5) übertragen, um die Rohdaten mit Hilfe der RAPID® Software Suite $6 \mathrm{zu}$ einem Video zu verarbeiten (RAPID = reporting and processing of images and data: Weitergabe und Verarbeitung von Bildern und Daten) (Given Imaging 2008, S. 2, 11).

\section{Abb. 5: Given Imaging® Workstation der UMG}

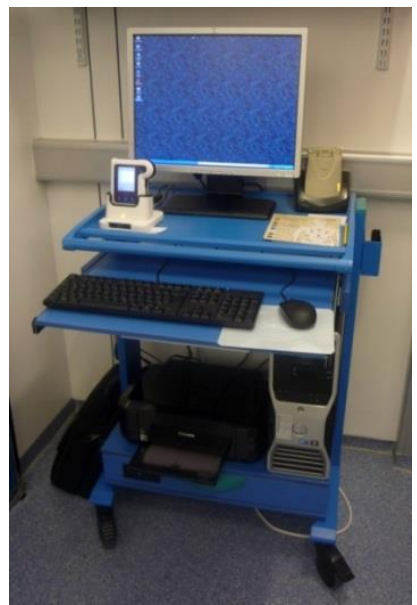

Diese Software (Abb. 6) ermöglicht einen Schnelldurchlauf der Bilder, die als auffällig gewertet und/ oder mit Blut versehen waren. Einzelbilder können im Vorwärts- und Rückwärtsmodus vom Programm abgespielt werden. Die Geschwindigkeit des Ablaufes kann konstant oder variabel angepasst werden. Zusätzlich besteht die Möglichkeit, mehrere Bilder gleichzeitig (1420 Stück) zu betrachten. Auffällige Befunde werden zur Erleichterung des Wiederfindens für die Befunderhebung markiert, mit einem Kommentar versehen und erscheinen als Thumbnail. Darüber hinaus erscheint das erste Bild der drei Abschnitte Magen, Duodenum und Zökum ebenfalls als Thumbnail (Fischer et al. 2004). Ein Balken oberhalb des Thumbnails, der diese Abschnitte in unterschiedlichen Farben darstellt (Magenpassage: blau, Dünndarmpassage: orange, Kolonpassage: grün), erleichtert die Übersicht. Mit Hilfe der vom Untersucher gesetzten Unterteilungen errechnet das Programm die Magen- und Dünndarmpassagezeit. Eine in das Programm integrierte Lokalisations-Software kann die jeweilige Kapselposition durch die auf die Bauchhaut geklebten Sensoren oder den SensorBelt ermitteln (Fischer et al. 2004). Die Lokalisation, der in der VKE gefundenen pathologischen Befunde, ist für den Untersucher sehr 
bedeutsam, da eine Bestimmung des jeweiligen Abschnittes des Gastrointestinaltraktes möglich ist. Zudem kann er bei nachfolgenden Interventionen einen Zugangsweg - von oral oder anal empfehlen (Fischer et al. 2004).

\section{Abb. 6: Pillcam-Platform}

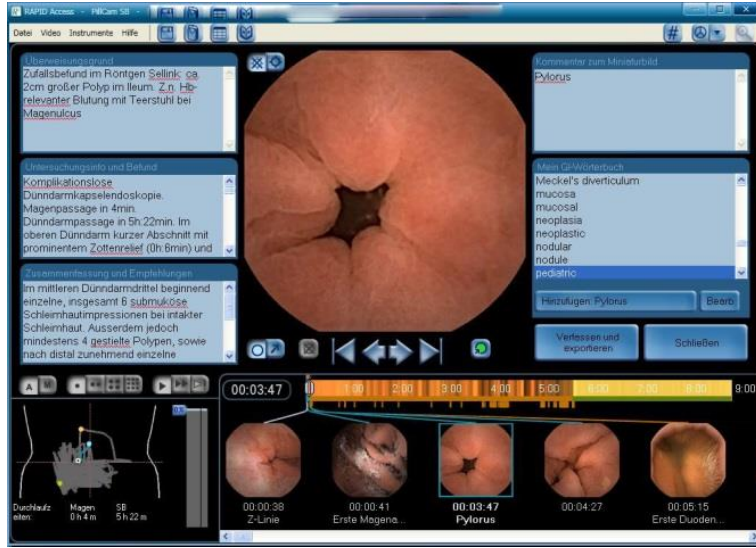

Darstellung der Passagezeiten anhand der Farbskala

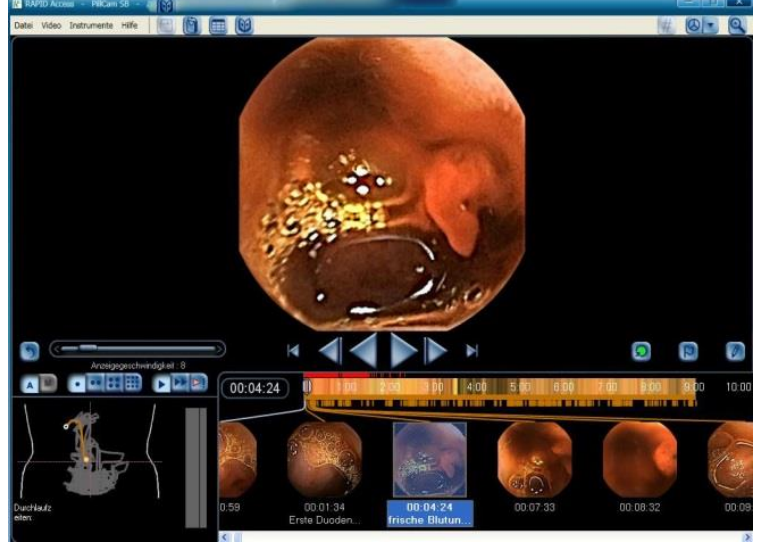

Darstellung des Blutungsdetektors (rote Linie oberhalb der Passageskala)

Für die schnelle Erkennung von Blutungen ist in der Software ein Blutungsdetektor (suspected blood indicator) vorhanden, der erst durch die Markierung des Dünndarms aktiviert wird. Dieser Detektor zeigt verdächtige rote Pixel, welche bei Farbveränderungen wie Blut oder großen Angiektasien auftreten (Güldütuna und Keuchel 2009, S. 43). Laut einer Studie von D'Halluin ersetzt diese Software jedoch nicht die Erfahrung und Genauigkeit eines Untersuchers, da falsch positive oder falsch negative Ergebnisse entstehen können (D'Halluin et al. 2005).

Bei Befunden, die schwer von normaler Schleimhaut zu unterscheiden sind, lässt ein BlaulichtModus (blue mode) eine digitale Filterung des Lichtspektrums $\mathrm{zu}$, um Pathologien besser $\mathrm{zu}$ erkennen. Mit Hilfe eines FICE-Programms (flexible intelligent colour enhancement) können Farben für die Befundung selektiert werden (Steinbrück et al. 2010).

Des Weiteren enthält die Software einen elektronischen Atlas mit pathologischen Bildern. Das erlaubt dem auswertenden Arzt, eigene Bilder mit den Bildern aus dem Atlas zu vergleichen. Die Möglichkeit, unklare Befunde mit Kollegen über Internetforen zu diskutieren, wird durch die digitale Speicherung der Untersuchung vereinfacht (Güldütuna und Keuchel 2009, S. 43).

Die Datensicherung sollte während der Befundung in regelmäßigen Abständen erfolgen. Die Daten lassen sich nach der Auswertung auf externen Festplatten, einzelnen DVDs oder auf Praxis- oder Klinikservern speichern (Güldütuna und Keuchel 2009, S. 44). 


\subsection{Indikationen, Kontraindikationen, Komplikationen}

Die Anzahl der möglichen Indikationen für die Videokapselendoskopie wächst stetig. Eine Aufzählung der Einsatzgebiete befindet sich in Tabelle 4.

Tabelle 4: Aktuelle Indikationen der Dünndarm-Videokapselendoskopie (Güldütuna und Keuchel 2009, S. 20)

1. obskure gastrointestinale Blutung oder unklare Eisenmangelanämie

2. Erkrankung oder Verdacht auf Morbus Crohn

3. Erkrankung oder Verdacht auf Sprue

4. Polyposis-Syndrome

5. Dünndarmtumoren/ Polypen

6. Therapiemonitoring nach Medikamentengabe oder nach Dünndarm- bzw. Stammzelltransplantationen

7. Abklärung von pathologischer Bildgebung

8. unklare Durchfallerkrankung

Stenosen im Gastrointestinaltrakt gelten als absolute Kontraindikation bei der VKE. Zudem ist zu beachten, dass vor Ausscheidung der Videokapsel keine MRT-Untersuchung erfolgen darf (Güldütuna und Keuchel 2009, S. 22 f).

Eine Stenose kann die Kapselretention im Dünndarm als Komplikation nach sich ziehen. Die Kapselretention ist seit der Internationalen Konferenz der Kapselendoskopie (ICCE 2005) definiert als Retention im Gastrointestinaltrakt, die länger als zwei Wochen besteht. Zudem gilt als Retention, wenn die Kapsel durch ein gezieltes Medikament, eine endoskopische und/ oder eine chirurgische Intervention geborgen wird (Cave et al. 2005). Um Stenosen ausschließen zu können, wurde bei den symptomatischen Patienten im Vorfeld eine Doppelkontrast-SellinkUntersuchung durchgeführt.

Mit Einführung der Pilotkapsel Patency-Kapsel von Given Imaging® kann die Durchgängigkeit der Videokapsel im Gastrointestinaltrakt überprüft werden. Bei einer Stenose löst sich die Kapsel selbstständig nach 30-100 Stunden im wässrigen Milieu auf. Diese Kapsel ist in der Form und Größe mit der PillCam SB 2 identisch. Die Patency-Kapsel besteht aus einer Radiofrequenz- 
spule, die 2x7 mm groß ist (Güldütuna und Keuchel 2009, S. 28). Diese von einer LaktoseBarium-Schicht umgebene Spule kann von einem externen Scanner erfasst werden. Die Eindringtiefe beträgt $20 \mathrm{~cm}$ (Güldütuna und Keuchel 2009, S. 28). Dank einer permeablen Membran kann Flüssigkeit nach ca. 30 Stunden in das Innere der Kapsel eintreten und die Laktose-Schicht auflösen, so dass am Ende nur noch die Spule und die Membranhülle übrig bleiben. Diese sollen selbst enge Stenosen passieren können (Güldütuna und Keuchel 2009, S. 28).

Wenn in der VKE keine Passage der Ileozökalklappe gesehen werden konnte, wurden die Patienten aufgefordert, sich innerhalb einer Woche nach der Untersuchung erneut in der Klinik vorzustellen, sollte die Kapsel nicht ausgeschieden werden. In diesem Fall erfolgte zunächst eine Röntgenübersichtsaufnahme des Abdomens zum Nachweis oder Ausschluss einer Stenose. Falls die Kapselendoskopie eine Obstruktion durch einen M. Crohn oder einen stenosierenden Dünndarmtumor nachweisen konnte, ist die Retention nicht nur als Komplikation, sondern auch als diagnostische Unterstützung anzusehen. Falls keine endoskopische Bergung möglich ist oder durch Einleitung abführender Maßnahmen kein spontaner Abgang erzielt werden kann, wird eine Operation durchgeführt.

$\mathrm{Zu}$ den relativen Kontraindikationen zählen Schwangerschaft, Schluckstörungen, Gastroparese und/ oder elektromedizinische Implantate sowie Voroperationen (Barkin und O'Loughlin 2004; Güldütuna und Keuchel 2009, S. 22).

Schluckstörungen gehören aufgrund der erhöhten Aspirationsgefahr $\mathrm{zu}$ den relativen Kontraindikationen. Deshalb ist eine sorgfältige Anamnese vor der Untersuchung zum Ausschluss einer Schluckstörung obligat. Im Falle einer Schluckstörung ist die Verabreichung der Videokapsel über eine endoskopische Applikation angeraten (Güldütuna und Keuchel 2009, S. 22).

Bei Patienten mit Verdacht auf Gastroparese oder Motilitätsstörungen des Magens (z.B. aufgrund von Diabetes mellitus, Morphinmedikation, Bettlägerigkeit oder anatomischer Veränderungen) wird die Anwendung des Real-Time Viewers empfohlen (Güldütuna und Keuchel 2009, S. 22). Bei Nichterreichen des Dünndarms ein bis zwei Stunden nach der VKEEinnahme kann die Einnahme eines Prokinetikums (Metoclopramid, Erythromycin) zur beschleunigten Passage führen. Eine Alternativmöglichkeit wäre die endoskopische Applikation der VKE in den Dünndarm (Güldütuna und Keuchel 2009, S. 22).

Neuere Studien konnten die relative Kontraindikation der elektromedizinischen Implantate (Schrittmacher oder Defibrillator) entkräften. Laut Bandorski und Elias (Bandorski et al. 2008; 
Elias und Toubia 2009) existiert keine Interaktion dieser medizinischen Geräte mit der Videokapsel. Betroffenen Patienten wird jedoch empfohlen, sich stationär einer videokapselendoskopischen Untersuchung $\mathrm{zu}$ unterziehen sowie Rücksprache mit dem jeweiligen Kardiologen zu halten. Zudem sollte der Schrittmacher oder Defibrillator nach der Kapselendoskopie überprüft werden (Güldütuna und Keuchel 2009, S. 22). Die Kapsel selbst kann durch elektromagnetische Felder wie die Telemetrie gestört werden (Bandorski et al. 2013).

\subsection{Durchführung}

Mit Zustimmung des Zentrums für Innere Medizin und der Kinderklinik konnten an der UMG die archivierten Patientendaten der Dünndarmkapselendoskopien von 203 Patienten im Zeitraum von 2006-2010 gesichtet werden. Patienten und Hausärzte wurden nicht kontaktiert.

\subsubsection{Kostenübernahme}

Alle privaten Krankenversicherungen übernahmen die Kosten für die VKE des Dünndarms bei korrekter Indikation unklare gastrointestinale Blutung bei vorher unauffälliger Gastroskopie und Koloskopie. Bei den gesetzlichen Krankenkassen konnte die Kostenübernahme nach entsprechender Antragstellung als Einzelfallentscheidung erwirkt werden. Voraussetzung war auch hier, dass Gastroskopie und Koloskopie zu keinem Ergebnis geführt hatten. Gelegentlich wurde die Genehmigung erst nach eingehender Prüfung durch den medizinischen Dienst der Krankenkassen bewilligt. Die VKE ist als individuelle Gesundheitsleistung (IGeL) jederzeit auch für gesetzlich Versicherte möglich. Die Materialkosten für eine Videokapsel betragen ca. $500 €$. 


\subsubsection{Aufklärungsgespräch}

Vor einer elektiven VKE-Untersuchung fand am Vortag ein ausführliches Aufklärungsgespräch statt. Durchgeführt wurde es von einem Arzt für Innere Medizin und/ oder Gastroenterologie.

Bei der Untersuchungsaufklärung wurden neben den bisher erhobenen Vorbefunden auch die Vorbereitung, die technische Durchführung und die Kostenübernahme besprochen bzw. beantragt. Die Patienten erhielten einen Aufklärungsbogen des proCompliance-Verlages. Diese Patientenaufklärung besteht aus Informationen über die Art der Spiegelung, die Vorbereitung, Kontraindikationen, Alternativmethoden, Risiken und Komplikationen. Im Anschluss füllen die Patienten mit Hilfe der Ärzte einen Fragebogen aus. Der Patient willigt schriftlich in diese Untersuchung ein. Vor Durchführung der VKE kontrolliert der Untersucher die Erklärung auf Genauigkeit und Unterschrift.

\subsubsection{Vorbereitung}

Nach Bestätigung der Kostenübernahme begann die Vorbereitung der VKE am Vortag. Die Patienten nahmen ein normales Frühstück ein, zum Mittagessen war Brühe ohne feste Nahrungsbestandteile gestattet. Ab der Mittagszeit begannen die Patienten zur Reinigung des Verdauungstraktes - abhängig von der Anordnung des untersuchenden Arzt - ein bis zwei Liter einer Lösung aus Wasser und ein bis zwei Beuteln Moviprep ${ }^{\circledR}$ oder Klean Prep ${ }^{\circledR}$ [aus Polyethylenglycol (Macrogol 3350)] zu trinken. Tee und Wasser (ca. zwei-drei Liter) waren am gesamten Tag vor der Untersuchung und bis zu zwei Stunden vor Untersuchungsbeginn gestattet. Die Patienten sollten 24 Stunden vor VKE-Einnahme keine feste Kost oder Kernobst zu sich nehmen. Eisentabletten waren bis mindestens drei Tage vor der Untersuchung gestattet, danach hätten diese zu Sichteinschränkungen während des Kapseldurchlaufes führen können. Das Gleiche galt für Antazida sowie für wasserunlösliche Kontrastmittel wie Bariumsulfat (May et al. 2010). 


\subsubsection{Anbringen des Datenrecorders}

Am nächsten Vormittag erschienen die Patienten in der Endoskopie. Die Anlage des Sensorengürtels mit der Datenrecordertasche wurde von den Ärzten in der gastroenterologischen Abteilung übernommen.

\subsubsection{Einnahme der Videokapsel}

Unter ärztlicher Aufsicht nahmen die Patienten die Videokapsel mit einem Schluck Wasser ein, nachdem sie gegebenenfalls einen Entschäumer (Simethicon) und/ oder als Prokinetikum MCPTropfen erhalten hatten. Bei Patienten mit Schluckbeschwerden wurde die Kapsel postpylorisch in den Bulbus duodeni über eine Schlinge oder durch einen speziell für die Videokapsel angefertigten Applikator eingebracht, welcher über das Endoskop gesteuert wurde.

Zwei Stunden nach dem Schlucken oder Applizieren der Kapsel konnten die Patienten klare Flüssigkeiten trinken, da davon ausgegangen wurde, dass die Kapsel bereits den Magen passiert hatte. Leichte Kost wie Nudeln, Brot oder Joghurt sowie die Einnahme von regulären Medikamenten war vier Stunden nach der Kapseleinnahme genehmigt. Die Kapsel hatte zu diesem Zeitpunkt in der Regel einen Vorsprung und wurde von den Nahrungsresten nicht beeinflusst.

\subsubsection{Auswertung der Untersuchungsergebnisse}

Die Dauer der Auswertung der Befunde der Patienten betrug im Durchschnitt 1,5 bis 3 Stunden. Sie erfolgte in der Regel noch am gleichen Tag nach Abgabe des Datenrecorders. Ein Arzt der gastroenterologischen Abteilung war hauptverantwortlich für die Auswertung. Die Vertretung übernahmen die jeweiligen Endoskopie-Assistenzärzte. 


\subsection{Statistik}

Grundlage dieser Arbeit war die deskriptive statistische Auswertung der Videokapselendoskopie-Untersuchungsbefunde, die von erfahrenen Ärzten der gastroenterologischen Abteilung am Universitätsklinikum Göttingen verfasst wurden.

Die statistische Erfassung der Daten erfolgte mit Hilfe des Tabellenkalkulationsprogramms Microsoft Excel 2007®.

Die Auswertung erfolgte mittels des Datenanalyse-Programms Statistica®. Durch die elementare Statistik konnten Mittelwertberechnungen, Medianwerte $(\overline{\mathrm{x}})$, die minimalen sowie maximalen Werte, das untere wie auch obere Quartil und die Standardabweichung $( \pm)$ berechnet werden. Die dichotomen Daten (0/1) wurden mit Hilfe von Häufigkeitstabellen bearbeitet. Diese Daten wurden mit Überlebensanalysen (Survival-Analysen), Box-and-Whisker Plots (Boxplots; Median, Interqartilsabstand, unteres/ oberes Quartil) sowie mit Scatter-Plot Diagrammen dargestellt.

Zur Untersuchung des Signifikanzniveaus wurde der p-Wert betrachtet. Dieser ist als signifikant definiert, wenn der Wert kleiner als 0,05 ist. Für diese Berechnung wurde der t-Test (Student's-tTest) oder bei mehr als zwei Kennwerten die Varianzanalyse (ANOVA: analysis of variance) herangezogen.

Für die Literaturrecherche wurde mit dem medizinischen Suchprogramm PubMed® gearbeitet, außerdem wurden gastroenterologische Standardwerke herangezogen. 


\section{Ergebnisse}

\subsection{Patientenkollektiv}

In dieser Arbeit wurden die Dünndarm-Videokapsel-Untersuchungen von 203 Patienten des Zentrums für Innere Medizin des Universitätsklinikums Göttingen vom 25.1.2006 bis zum 16.11.2010 analysiert.

Bei vier Patienten wurde die Kapselendoskopie zweimal durchgeführt. Diese Untersuchungen werden in der Statistik einzeln berechnet. In zwei Fällen machten auffällige Befunde eine weitere Kontrolluntersuchung nötig, ein weiteres Mal musste die Untersuchung aufgrund von störender Verstuhlung wiederholt werden. Beim vierten Patienten war hingegen ein Kapseldefekt verantwortlich.

Das Patientengut bestand aus 118 männlichen (58\%) und 85 weiblichen Patienten (42\%). Der Altersdurchschnitt des Patientenkollektivs lag bei $58( \pm 19,5)$ Jahren.

Das Durchschnittsalter der männlichen Patienten lag bei 59 ( \pm 19$)$ Jahren. Die Altersspanne der männlichen Patienten lag zwischen 8 und 89 Jahren, der Interquartilsabstand zwischen 48 und 72 Jahren, der Median, betrug 64 Jahre. Das Durchschnittsalter des weiblichen Patientenkollektivs betrug $56( \pm 21)$ Jahre. Die jüngste Patientin war 11 Jahre und die älteste Patientin 90 Jahre alt. Bei den weiblichen Patienten war der Interquartilsabstand größer, er lag zwischen 41 und 73 Jahren. Der Median lag bei 60 Jahren. Die Altersstatistik ist aus der Abb. 7 ist ersichtlich.

\section{Abb. 7: Boxplot für Patientenalter, gruppiert nach Geschlecht}

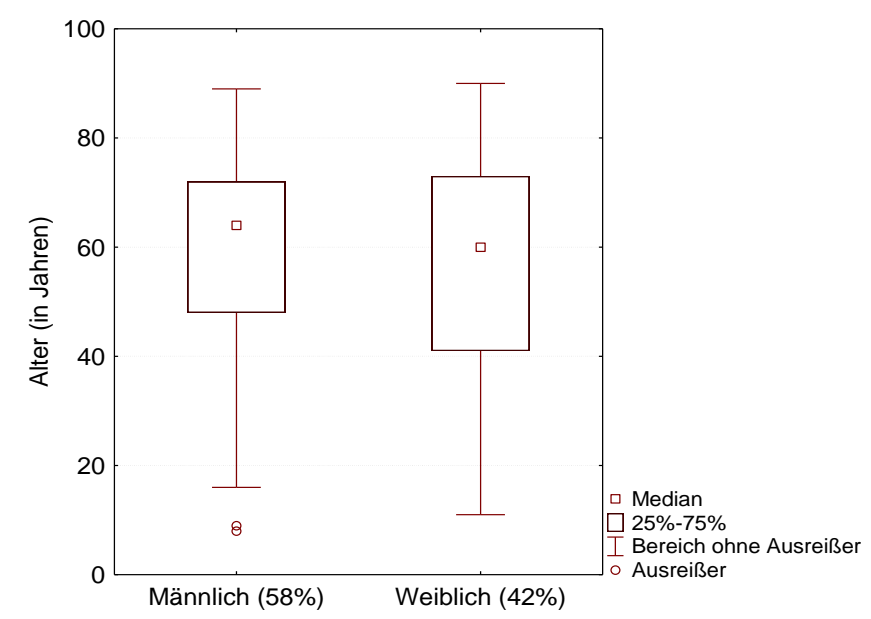


Tabelle 5: Charakteristik der Patientenpopulation $(n=203)$

\begin{tabular}{lll}
$\begin{array}{l}\text { Geschlecht (Frauen/ Männer) } \\
\text { Durchschnittsalter (Jahre) }\end{array}$ & 58 & $118(58 \%)$ \\
VKE-Durchführung (Pat.) & $\begin{array}{l}154(76 \%) \text { stationär } \\
\text { Altersspanne: } 8 \text { - } 90\end{array}$ & $48(23,5 \%)$ ambulant \\
Krankenhaus- & $\begin{array}{l}\text { X }=10,6 \text { Tage } \\
\text { aufenthaltsdauer }\end{array}$ & 3 Pat. $(1,5 \%)$ verstarben \\
BMI (I = unter-, II = normal- & I: $15(7 \%)$ Pat. 1 Tag, Max.: 47 Tage \\
III = übergewichtig, & III: $85(42 \%)$ Pat. & II: $75(37 \%)$ Pat. \\
IV = Angaben fehlten) & & IV: $28(14 \%)$ Pat. \\
\hline
\end{tabular}

Bei 154 Patienten (75,9\%) erfolgte die VKE-Untersuchung im Rahmen des stationären Aufenthaltes. Bei 48 Patienten (23,6\%) wurde die Kapselendoskopie ambulant durchgeführt. Für einen Patienten $(0,5 \%)$ liegen diesbezüglich keine Angaben vor.

Die durchschnittliche Krankenhausaufenthaltsdauer lag bei den stationären Patienten bei 10,6 Tagen inklusive diagnostischer Voruntersuchungen, Durchführung der VKE und Therapiebeginn und/ oder Nachuntersuchungen.

Drei Patienten verstarben während des stationären Aufenthaltes an ihren Grundleiden, bei allen lag die Indikation unklare gastrointestinale Blutung vor.

Der Body-Mass-Index (BMI) des Patientenkollektivs wurde in drei Kategorien unterteilt:

1. Patienten mit einem BMI $<18,5 \mathrm{~kg} / \mathrm{m}^{2}$ galten als untergewichtig,

2. Patienten mit einem BMI zwischen $18,5-25 \mathrm{~kg} / \mathrm{m}^{2}$ galten als normalgewichtig,

3. Patienten mit einem $\mathrm{BMI}>25 \mathrm{~kg} / \mathrm{m}^{2}$ galten als übergewichtig.

Jeweils 15 Patienten (7,4\%; 7 Frauen und 8 Männer) waren laut BMI untergewichtig. Normalgewichtig waren 75 Patienten (37\%; 31 Frauen und 44 Männer). Als übergewichtig galten 85 Patienten (41,9\%; 34 Frauen und 51 Männer). Der Median der gesamten BMI-Werte lag bei 24,8 .

Bei 28 Patienten (13,8\%; 13 Frauen und 15 Männer) konnte kein BMI-Wert bestimmt werden, da sowohl Körpergröße als auch das Gewicht der Patienten in den Patientenakten fehlten. 


\subsection{Indikationen}

Folgende Indikationen wurden bei dem Göttinger Patientenkollektiv für die Kapselendoskopie gestellt (Tabelle 6).

Die meisten Patienten hatten mehrere Indikationen in der Untersuchungsanforderung. Bei der Auswertung wurde nur die Hauptindikation jedes Patienten berücksichtigt.

Tabelle 6: Indikationen der Patienten $(n=203)$

\begin{tabular}{llll}
\hline & insgesamt $(\%)$ & $\begin{array}{l}\text { Frauen }(\%): \\
\mathrm{n}=85\end{array}$ & $\begin{array}{l}\text { Männer (\%): } \\
\mathrm{n}=118\end{array}$ \\
\hline unklare gastrointestinale Blutung & $92(45,3)$ & $32(38)$ & $60(51)$ \\
unklare Anämie & $23(11,3)$ & $11(13)$ & $12(10,2)$ \\
unklare abdominelle Schmerzen & $49(24,1)$ & $24(28,1)$ & $25(21,1)$ \\
Verdacht auf Morbus Crohn & $8(4)$ & $3(3,5)$ & $5(4,2)$ \\
Komplikation bei Morbus Crohn & $5(2,5)$ & $2(2,4)$ & $3(2,5)$ \\
unklare Diarrhoe & $13(6,4)$ & $6(7)$ & $7(6)$ \\
Polyp- und Tumorsuche & $11(5,4)$ & $6(7)$ & $5(4,2)$ \\
unklares rezidivierendes & $1(0,5)$ & $1(1)$ & 0 \\
Erbrechen & $1(0,5)$ & & $1(0,8)$ \\
Eiweißverlustsyndrom & & & \\
\hline
\end{tabular}

\subsection{Voruntersuchungen}

Alle 203 Patienten erhielten vor der VKE Laboruntersuchungen und Abdomen-Sonografien sowie mindestens eine Gastroskopie und Koloskopie, meist kombiniert mit Biopsien.

Darüber hinaus erhielten 116 Patienten $(57,1 \%)$ ergänzende Untersuchungen. Nicht immer lagen bei dem Göttinger Patientenkollektiv alle Befunde der durchgeführten Untersuchungen zum Zeitpunkt der Kapselendoskopie vor.

Bei den Frauen und Männern, die unter einer unklaren Anämie litten, wurden neben dem Blutbild mit Differenzierung auch Ferritin, Folsäure und Vitamin B12 im Serum bestimmt. 
Bei fünf Patienten (2,5\%) erfolgte zusätzlich die Bestimmung von Tumormarkern wie CEA, CA 19-9 und Chromogranin A. Eine Zöliakie/ Sprue sollte bei 16 Patienten (8\%) durch Nachweis von Antikörper-Suchtesten wie Gliadin- und Transglutaminase-Autoantikörper nachgewiesen oder ausgeschlossen werden.

Bei drei Patienten (1,5\%) wurde eine Beckenkammpunktion wegen des Verdachts auf eine hämatologische Systemerkrankung durchgeführt. Zwei Patienten (1\%) erhielten eine HNOärztliche Vorstellung, um eine Blutungsursache im Oropharynx auszuschließen. Zur Erkennung einer vaginalen Blutung wurden fünf Frauen (6\%) gynäkologisch untersucht. Bei den Stuhluntersuchungen war die Suche nach okkultem Blut dominierend (Hämoccult-Test), seltener wurde nach darmpathogenen Keimen gesucht.

Zur Abklärung von unspezifischen Symptomen wie Gewichtsverlust, unklaren Diarrhoen, Erbrechen und Inappetenz, bei Verdacht auf Sprue (Zottenatrophie) oder einer Infektion mit dem Erreger Tropheryma whipplei wurden tiefe Dünndarmbiopsien durchgeführt. Zusätzlich wurden bei Patienten mit Diarrhoe unklarer Genese, nach Ausschluss einer infektiösen Genese durch mikroskopische Stuhluntersuchungen, Koloskopien mit Stufenbiopsien vorgenommen. Es konnte somit das Vorhandensein einer mikroskopischen Colitis (lymphozytäre oder kollagene Colitis) oder einer chronisch entzündlichen Darmerkrankung wie Colitis ulcerosa oder Morbus Crohn abgeklärt werden. Zum Ausschluss einer exokrinen Pankreasinsuffizienz bei Malabsorptionsstörungen wurden die Pankreaselastase und das Stuhlgewicht bestimmt. Bei Verdacht auf chronisch entzündliche Darmerkrankungen wurde das fäkale Calprotectin zum Ausschluss bzw. Nachweis verwendet.

Bei 16 Patienten (8\%) wurde eine Hepatitis-Serologie veranlasst. Im Rahmen einer Laktoseintoleranz-Diagnostik erfolgte bei zwölf Patienten $(6 \%)$ ein Wasserstoff $\left(\mathbf{H}_{2}\right)$-LaktuloseAtemtest und bei drei Patienten $(1,5 \%)$ ein Glukose-H H $_{2}$-Atemtest, um eine bakterielle Fehlbesiedlung des Dünndarms zu erkennen.

Häufig wurden im Vorfeld zur Kapselendoskopie bildgebende Untersuchungen veranlasst. Bei 28 Patienten (13,8\%) mit Blutungen oder ungeklärten Magen-Darm-Beschwerden fand neben dem Ultraschall des Abdomens eine CT-Untersuchung des Abdomens mit Kontrastmittelapplikation statt, um pathologische Organmanifestationen oder Blutungen zu detektieren. Ausgehend vom Gesamtpatientenkollektiv waren fünf Patienten (2\%) an einem M. Crohn erkrankt, davon erhielten drei Patienten (60\%) eine Enteroklysma-Untersuchung, abhängig von den Vorbefunden der Gastroskopie und Koloskopie. Von den acht Patienten (4\%) mit einem Verdacht auf eine M. Crohn-Erkrankung wurde bei einem Patienten (12,5\%) eine 
Enteroklysma-Untersuchung durchgeführt. 21 Patienten (9\%) erhielten diese letztgenannte Untersuchung ohne einen Bezug auf eine entzündliche Darmerkrankung. Die Art der Untersuchung war an das Patientenalter gebunden: Wegen der hohen Strahlenbelastung erhielten Patienten im fertilen Alter eine MRT-Untersuchung nach Sellink, bei älteren Patienten fanden Röntgen-gestützte Untersuchungen statt.

Lediglich bei drei Patienten (1,5\%) wurde eine Magen-Darm-Passage zur Abklärung von Stenosen durchgeführt. Bei weiteren drei Patienten (1,5\%) erfolgte eine 18-FDG-PETUntersuchung zum Nachweis bzw. Ausschluss eines erhöhten Stoffwechsels bei Tumorzellen.

Bei elf Patienten (5\%) mit abdominellen Schmerzen wurde zusätzlich zu den endoskopischen Untersuchungen vor der VKE eine ERCP zum Ausschluss einer biliären Genese durchgeführt. In vier Fällen erhielten die Patienten (2\%) eine Laparoskopie bzw. eine Laparotomie mit Inspektion des Dünndarms.

Bei den 203 untersuchten Patienten liegt die durchschnittliche Rate an Voruntersuchungen bei 4,3 Untersuchungen pro Patient. 


\subsection{Videokapselendoskopie}

\subsubsection{Kapselaufnahme}

189 Patienten (93\%) konnten die Videokapseln mit Hilfe einer kleinen Menge Wasser schlucken. Bei 14 Patienten (7\%) wurden die Kapseln während einer ÖGD appliziert. Diese Methode wurde fünfmal bei Kindern und Jugendlichen im Alter zwischen acht und 16 Jahren $(\overline{\mathrm{X}}=11$ Jahre $)$ angewandt, die entweder an einem Polyposis-Syndrom litten oder bei denen der Verdacht auf Morbus Crohn oder intestinalen Eiweißverlust bestand. Die anderen neun Kapselapplikationen erfolgten bei erwachsenen Patienten, die Altersspanne reichte von 36 bis 86 Jahren ( $\overline{\mathrm{x}}=68$ Jahre). Zwei dieser neun Patienten hatten eine Magenresektion nach Billroth II in ihrer Anamnese, die anderen konnten die Kapsel aufgrund physischer oder psychischer Schluckstörungen nicht schlucken.

\subsubsection{Verwendbarkeit der Kapsel}

Die Videokapseln konnten bei 145 Patienten (71,3\%) komplett bis zum Aufzeichnungsende ausgewertet werden, jedoch befand sich manchmal die Kapsel zu diesem Zeitpunkt manchmal noch im Dünndarm. Bei diesen Patienten war aufgrund einer guten Dünndarmsauberkeit eine optimale Auswertung möglich. Bei 51 Patienten (25,2\%) konnten die VKE-Ergebnisse nur teilweise befundet werden. Der Dünndarm dieser Patienten war entweder zu stark mit Nahrungsresten verschmutzt bzw. alte oder frische Blutauflagerungen behinderten die Schleimhautbeurteilung, obwohl sich alle Patienten vor der VKE einer Darmlavage unterzogen hatten. Bei sieben Patienten $(3,5 \%)$ war die VKE erfolglos. Einmal wies die Videokapsel einen technischen Defekt oder einen Übertragungsfehler auf. Zwei weitere Kapselendoskopien konnten wegen starker Stuhlverschmutzung nicht befundet werden, da die Sicht der Kamera sehr einschränkt war. Die restlichen vier Videokapseln verblieben während der Aufnahmezeit im Magen. 
Die Verwertbarkeit der Videokapselendoskopien des Patientenkollektivs ist in der Abb. 8 grafisch dargestellt. Die Auswertung der Kapselendoskopien wurde unterteilt in: 1 = verwertbar, 2 = inkomplette Auswertung, 3 = nicht verwertbar.

\section{Abb. 8: Verwertbarkeit der Videokapsel}

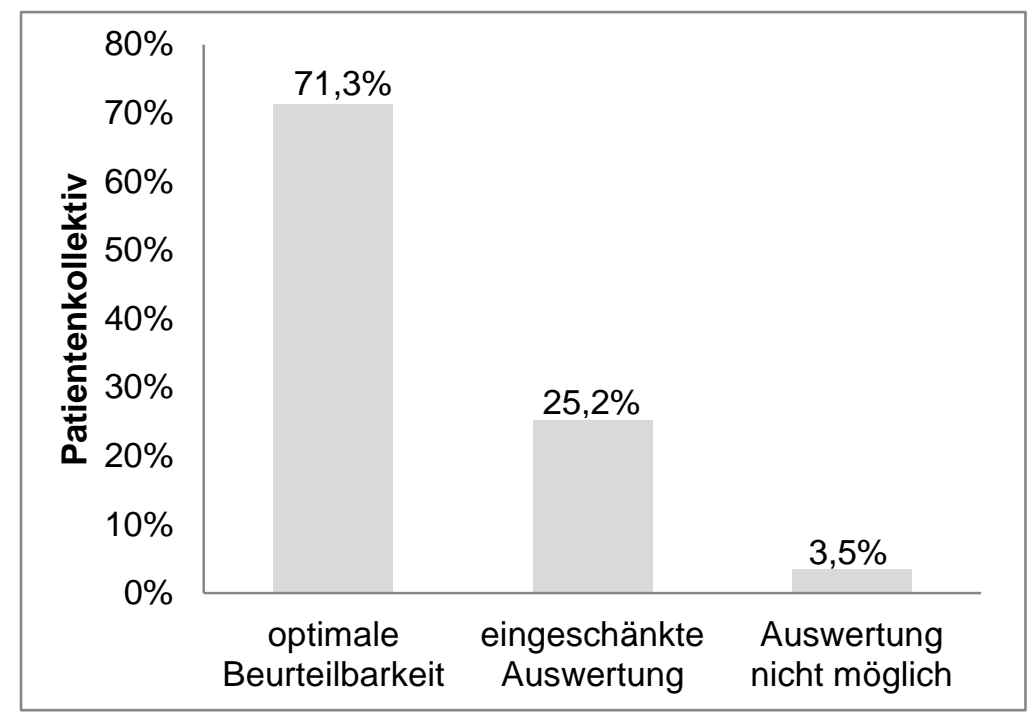

\subsubsection{Magen- und Dünndarmtransitzeit}

Die Magen- und Dünndarmtransitzeit konnte durch den Zeitzähler der Given Imaging®Software berechnet werden, indem die auswertenden Ärzte anhand der Bilder den Eintritt in den Magen sowie das erste Dünndarm- und das erste Dickdarmbild bestimmten.

Die exakte Passagezeit des Magens konnte bei 173 Patienten (85,2\%) bestimmt werden. Bei 26 Patienten (12,8\%) fehlte die Zeitangabe der Magenpassagezeit in den KapselendoskopieBefunden, mehr als die Hälfte $(53,8 \%)$ dieser Patienten $(n=14)$ erhielt eine Kapselapplikation in den Dünndarm.

Bei weiteren vier Patienten (2\%) verblieb die Videokapsel über den gesamten Zeitraum von acht Stunden im Magen. Diese Patienten wurden von der Auswertung ausgeschlossen.

Der Median der Magen-Transitzeit liegt bei 21 Minuten ( $\pm 69,9$ Minuten), wobei das Minimum bei einer Minute und das Maximum bei 462 Minuten liegt. 


\section{Abb. 9: Magenpassagezeit}

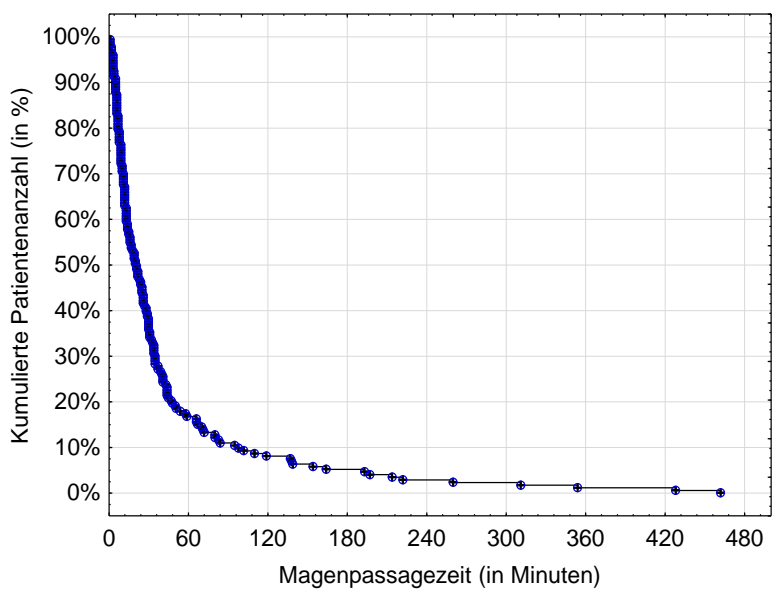

Kaplan-Meier-Kurve der Patientenanzahl (\%) unter Berücksichtigung der Magenpassagezeit (Min.)

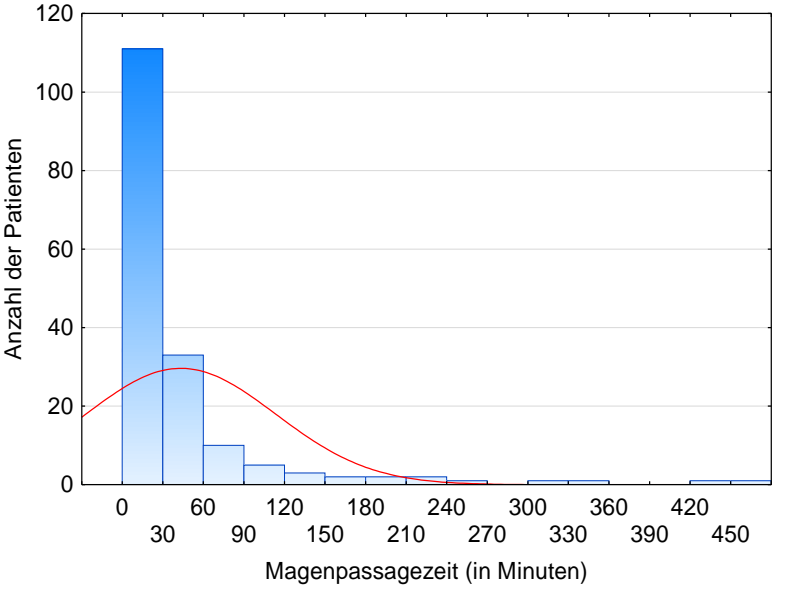

Histogramm der Patientenanzahl in Bezug auf die Magenpassagezeit (Min.)

Aus der Kaplan-Meier-Kurve der Abb. 9 ist ersichtlich, dass 50\% der Videokapseln der 173 Patienten schon nach 19 Minuten den Magen durchlaufen hatten. Bei Erreichen der 120Minuten-Marke befanden sich nur noch 14 Kapseln (8\%) im Magen. Nach 462 Minuten hatten alle 173 Videokapseln den Magen passiert.

Das Histogramm der Abb. 9 zeigt eine linksgipflige Verteilung. Dabei durchquerten 111 Videokapseln $(64,2 \%)$ den Magen bereits innerhalb von 30 Minuten. Weitere 33 Videokapseln (19\%) schafften eine Passagezeit von 60 Minuten.

Eine Signifikanz zwischen längeren Magenpassagezeiten bei höherem Alter $(p=0,9)$ oder höheren BMI-Werten konnte nicht festgestellt werden ( $p=0,9)$, siehe Abb. 10. 
Abb. 10: Scatterplot der Magenpassagezeit
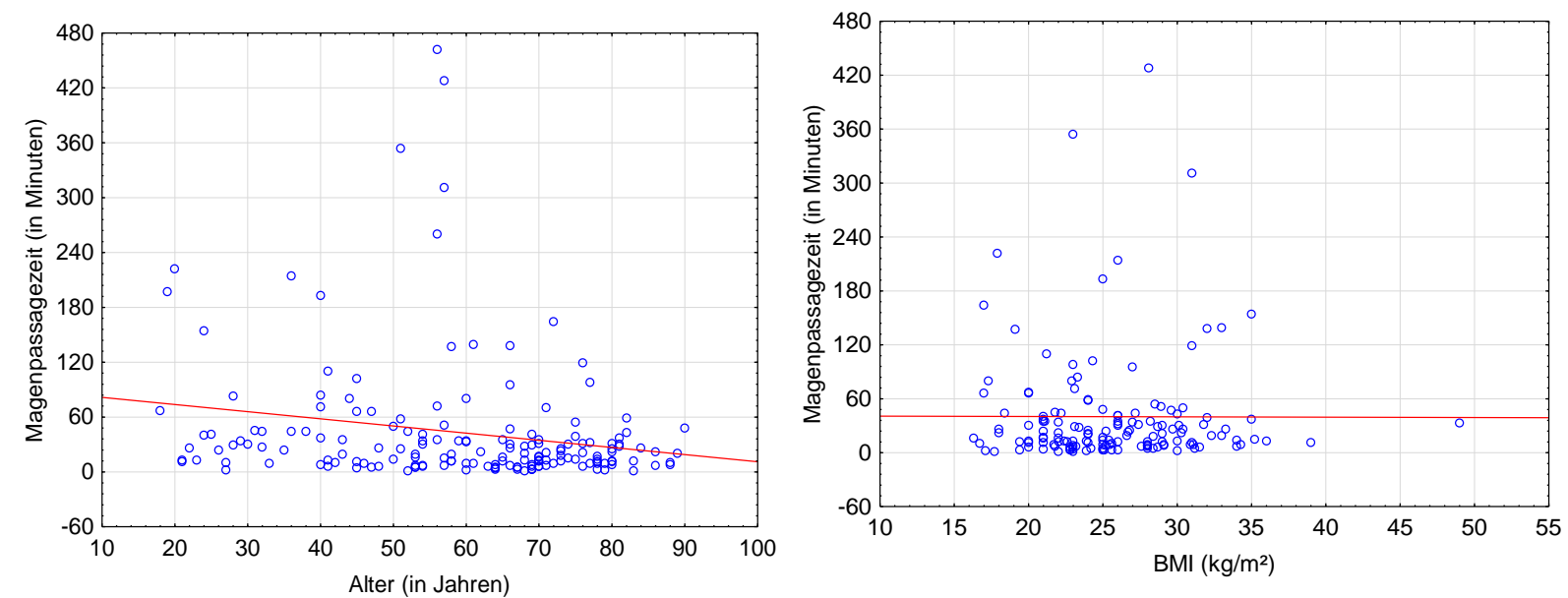

Darstellung der Magenpassagezeit (Min.)

Darstellung der Magenpassagezeit (Min.) unter Berücksichtigung des Patientenalters unter Berücksichtigung der BMI-Werte der Patienten

\section{Abb. 11: Boxplot der Magenpassagezeit - I}

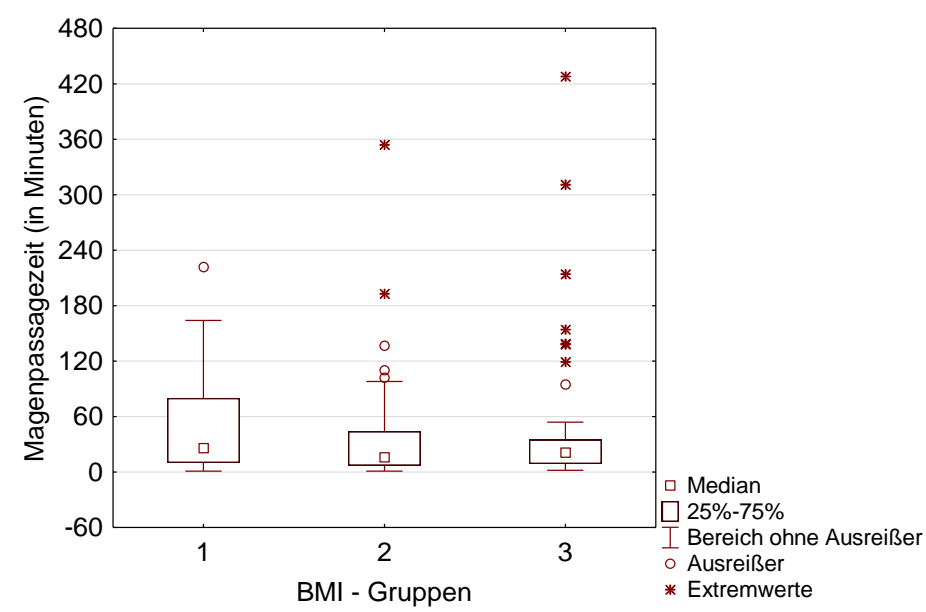

Darstellung der Magenpassagezeit (Min.) unter Berücksichtigung der BMI-Gruppen der Patienten

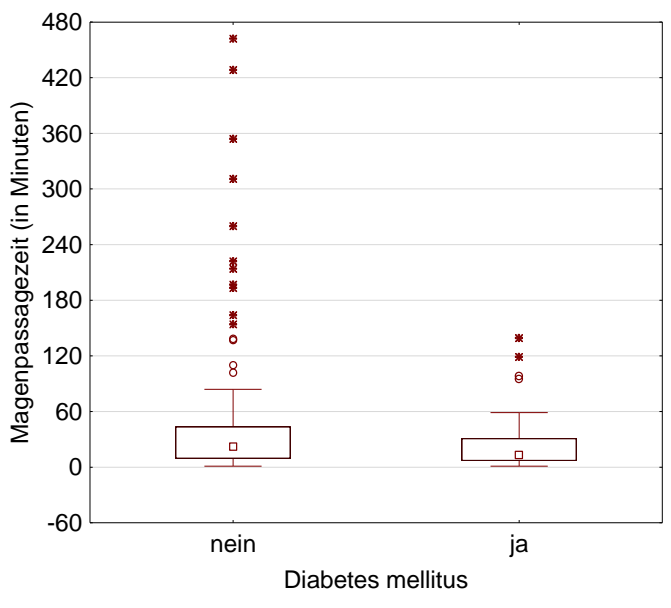

Darstellung der Magenpassagezeit (Min.) unter Berücksichtigung der an Diabetes mellitus erkrankten Patienten (29/173) des Kollektivs 


\section{Abb. 12: Boxplot der Magenpassagezeit - II}

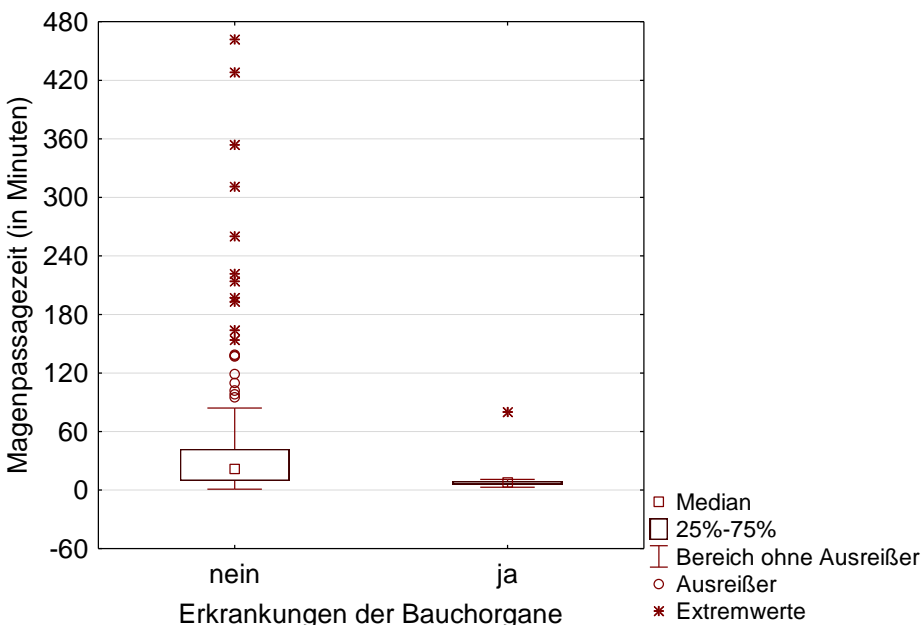

Darstellung der Magenpassagezeit (Min.) unter

Berücksichtigung der Bauchorganerkrankungen

(9/173) des Patientenkollektivs

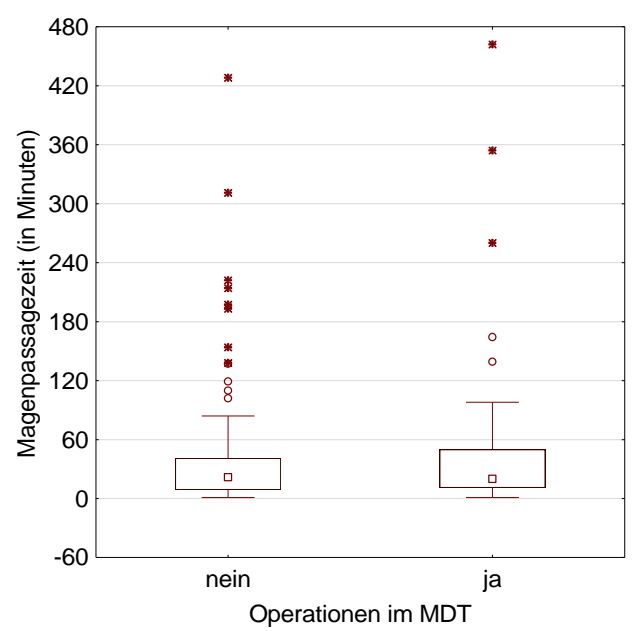

Darstellung der Magenpassagezeit (Min.) unter Berücksichtigung der Magen-Darm-Trakt-Operationen (39/173) der Patienten

Eine Korrelation zwischen einer bestimmten Magenpassagezeit und den unterschiedlichen BMI-Gruppen sowie den Diabetes mellitus - Patienten, den Patienten mit Erkrankungen der Bauchorgane oder Operationen im Magen-Darm-Trakt kann aus Abb. 11 und Abb. 12 nicht entnommen werden. Lediglich lässt die BMI-Gruppe 3 in Abb. 11 mehr maximale Abweichungen in der Magenpassagezeit erkennen, als in der BMI-Gruppe 1 und 2 vorhanden sind. Die Vermutung, dass Patienten mit Diabetes mellitus oder mit Erkrankungen des Magen-DarmTraktes eine längere Magenpassagezeit aufweisen, kann in Abb. 11 und Abb. 12 nicht bestätigt werden. Abb. 12 zeigt die Patienten, die anamnestisch im Magen-Darm-Trakt operiert worden sind im Vergleich zu der nicht operierten Patientengruppe. Die operierten Patienten weisen einen größeren Interquartilsabstand auf (operierte Patienten: 11-50 Minuten, nicht operierte Patienten: 9-41 Minuten). Die Maximalwerte der Magenpassage liegen ebenfalls bei den operierten Patienten höher: 462 Minuten (nicht operierte Patienten: 428 Minuten) bei einer Gesamtbatterielaufzeit von 480 bis 540 Minuten.

Die Dünndarmtransitzeit konnte bei 170 Probanden $(83,7 \%)$ ermittelt werden. Die Gesamtaufzeichnungszeit des Dünndarms lag zwischen 18 und 522 Minuten. Der Median dieser Patienten lag bei 245 Minuten ( $\pm 96,6$ Minuten). In diese Berechnung wurden 33 Patienten 
(16,3\%) nicht einbezogen. 22 Patienten von ihnen (10,8\% des Gesamtkollektivs) hatten eine längere Transitzeit als acht oder neun Stunden (abhängig von der Batteriekapazität der Videokapsel-Pillcam SB oder SB2). Bei vier Patienten (2\%) verließ die Kapsel während der Aufzeichnung den Magen nicht. Angaben zur Dünndarmpassagezeit fehlten bei sieben Patienten $(3,5 \%)$.

Abb. 13: Dünndarmpassagezeit

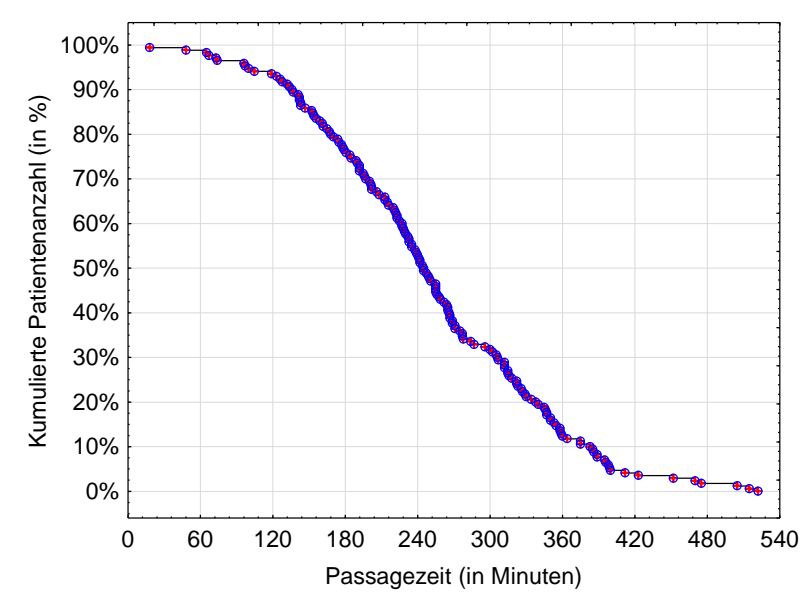

Kaplan-Meier-Kurve der Patientenanzahl (\%) unter Berücksichtigung der Dünndarmpassagezeit (Min.)

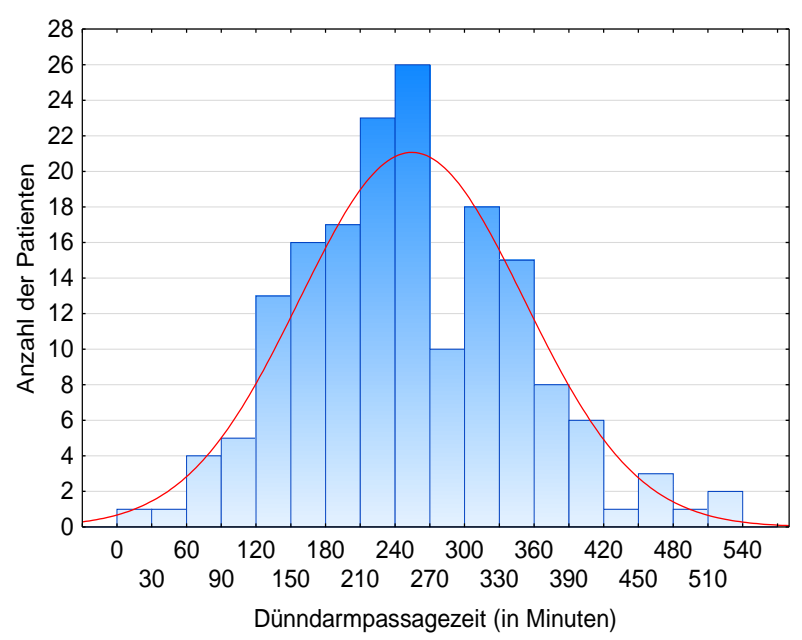

Histogramm der Patientenanzahl in Bezug auf die Dünndarmpassagezeit (Min.)

In der Abb. 13 sind alle 170 komplett auswertbaren Fälle dargestellt. Aus der Kaplan-MeierKurve der Abb. 13 lässt sich ein exponentieller Zerfall entnehmen. Bei 85 Patienten (50\%) durchquerten die Videokapseln den Dünndarm innerhalb von 242 Minuten. Nach 300 Minuten gelangten 53 Kapseln (31\%) und nach 420 Minuten 7 Videokapseln (4\%) in den Dickdarm. Alle 170 dokumentierten Videokapseln erreichten die Ileozökalklappe nach maximal 522 Minuten. Das Histogramm der Abb. 13 weist eine symmetrische Verteilung auf. Der Hauptteil der Patienten hatte eine Dünndarmpassagezeit von 180 bis 330 Minuten. Die restlichen Patienten hatten eine kürzere oder längere Passagezeit.

Aus Abb. 14 ist ersichtlich, dass die unterschiedlichen Dünndarmpassagezeiten nicht mit einem bestimmten Alter oder BMI-Wert korrelieren. 
Abb. 14: Scatterplot der Dünndarmpassagezeit

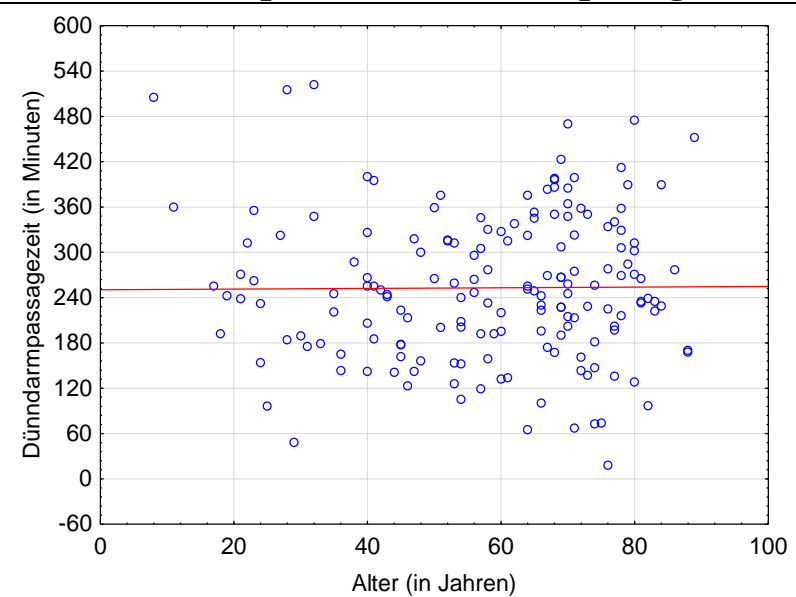

Darstellung der Dünndarmpassagezeit (Min.) unter Berücksichtigung des Patientenalters

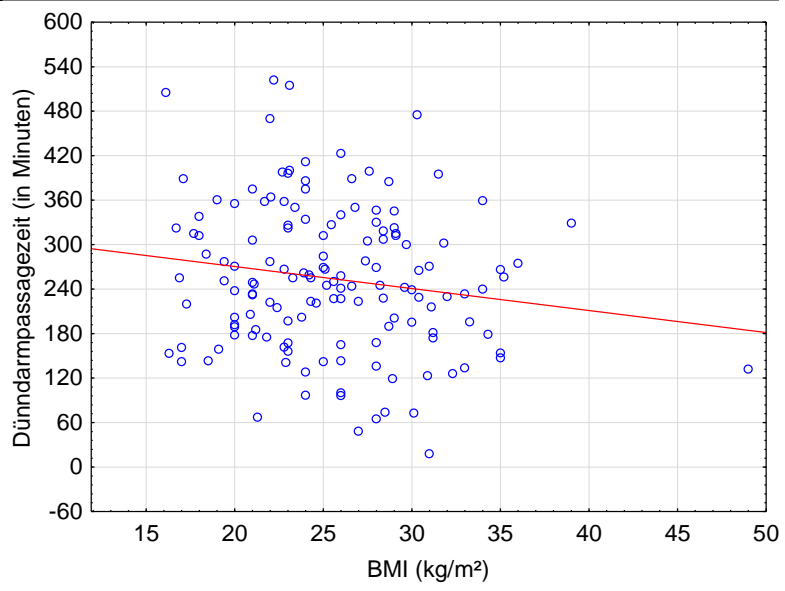

Darstellung der Dünndarmpassagezeit (Min.) unter Berücksichtigung der BMI-

Werte der Patienten

Abb. 15 und Abb. 16 zeigen keine verlängerte Dünndarmpassagezeit bei erhöhtem BMI, Diabetes mellitus oder Bauchorganerkrankung sowie nach Operationen des Magen-DarmTraktes. 
Abb. 15: Boxplot der Dünndarmpassagezeit
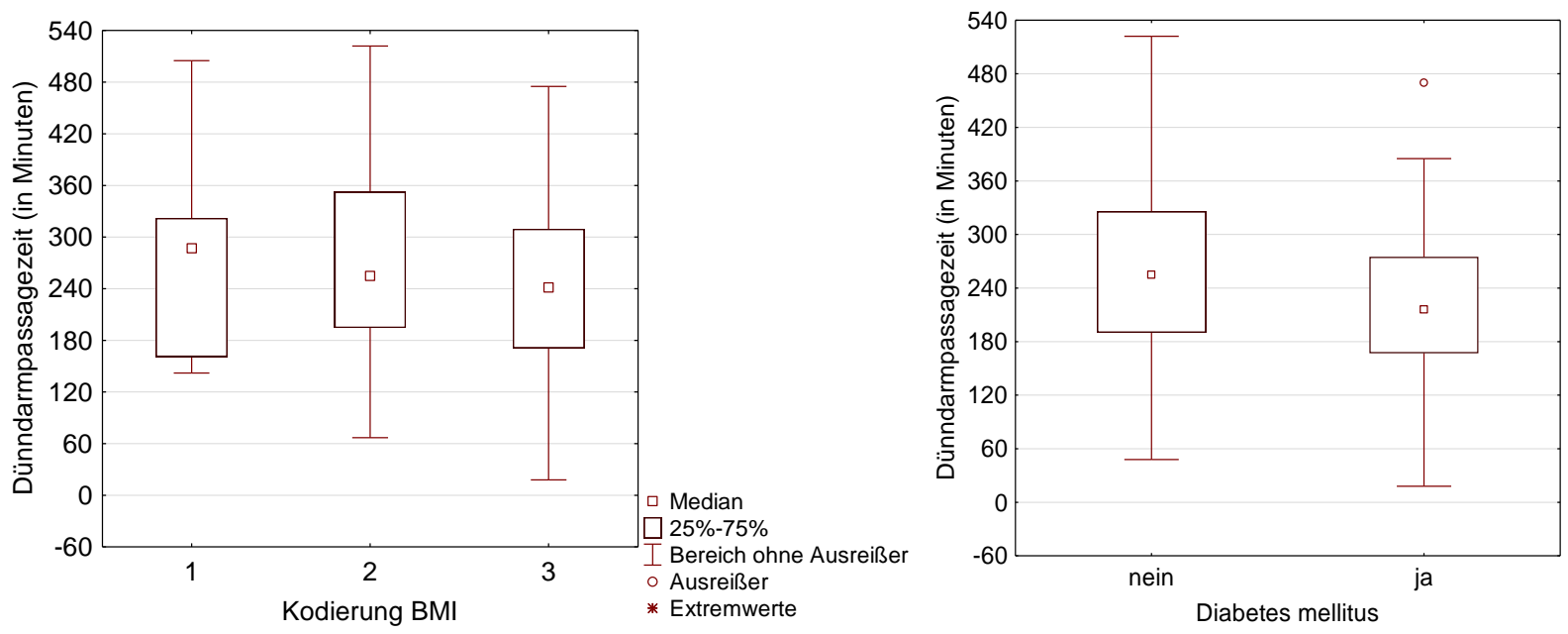

Darstellung der Dünndarmpassagezeit (Min.)

Darstellung der Dünndarmpassagezeit unter Berücksichtigung der BMI-Gruppen (Min.) unter Berücksichtigung der an Diabetes mellitus erkrankten Patienten (27/170) des Patientenkollektivs

\section{Abb. 16: Boxplot der Dünndarmpassagezeit}

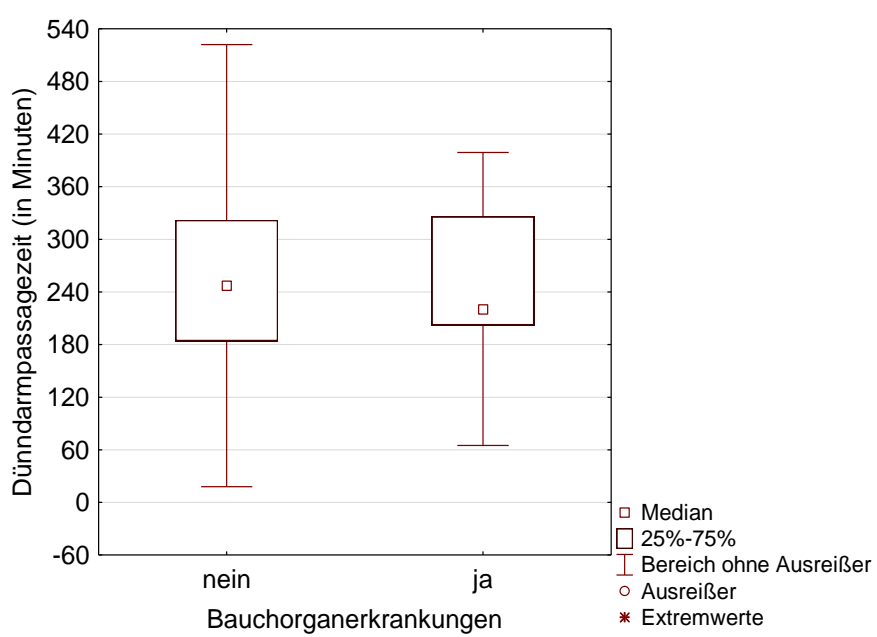

Darstellung der Dünndarmpassagezeit

(Min.) unter Berücksichtigung der

Bauchorganerkrankungen (11/170) des Patientenkollektiv

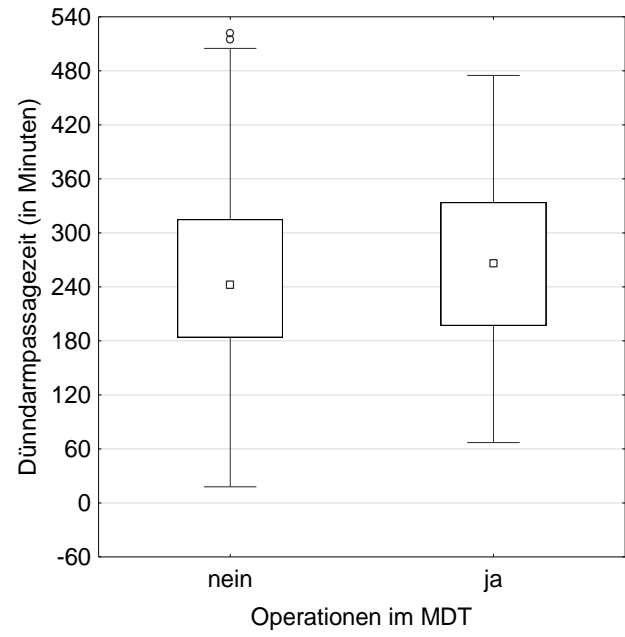

Darstellung der Dünndarmpassagezeit (Min.) unter Berücksichtigung der MDT-Operationen (37/170) der Patienten 


\subsubsection{Passage Ileozökalklappe}

Die Videokapsel ermöglicht die Darstellung der Passage durch die Ileozökalklappe. Bei 170 Patienten des Patientenkollektivs $(83,7 \%)$ ereignete sich die Passage innerhalb der Batterielaufzeit von acht oder neun Stunden. Bei 29 Patienten (14,3\%) passierten die Kapseln erst nach Ablauf der Batteriebetriebszeit spontan die Verbindungsklappe zwischen Dünn- und Dickdarm. Bei Patienten, bei denen die Kapseln mehrere Tage im Verdauungstrakt verblieben, wurde eine Röntgen-Abdomen-Untersuchung veranlasst. Damit sollte festgestellt werden, ob die Kapsel die Ileozökalklappe durchlaufen hat. Bei zwei dieser Patienten wurde die Videokapsel im kleinen Becken/ Sigmabereich gesehen. Es erfolgte ein spontaner analer Kapselabgang. Bei den anderen Patienten konnte die Kapsel radiologisch nicht nachgewiesen werden. Bei vier anderen Patienten (2\%) zeigte diese Untersuchung, dass sich die Videokapsel als metallischer Fremdkörper im rechten Unterbauch des Verdauungstraktes positioniert hatte. 50 Tage nach der VKE konnte bei dem ersten dieser vier Patienten - einem Morbus CrohnPatienten mit abdomineller Abwehrspannung und abdomineller Resistenz - die Videokapsel mit Hilfe einer Ileokoloskopie aus dem terminalen Ileum geborgen werden. Dabei wurde eine hochgradige Stenosierung der Ileozökalklappe festgestellt.

Bei dem zweiten Patienten (Indikation FAP; beschwerdefrei) wurde die Kapsel, die in der Röntgen-Abdomen-Abbildung noch oral der Ileozökalklappe lag, durch die Darmlavage, die zur Ileokoloskopie-Vorbereitung stattfand, ins Kolon mobilisiert. Endoskopisch konnte dann die Kapsel neun Tage nach der Kapselapplikation aus dem Rektum entfernt werden.

Der dritte Patient (Symptom unklare abdominelle Schmerzen) konnte die Kapsel - nach erfolglosem endoskopischen Bergungsversuch - zehn Tage nach der VKE durch medikamentöse Abführmaßnahmen ausscheiden. Durch die VKE wurde bei diesem Patienten die Diagnose M. Crohn gestellt.

15 Tage nach der VKE misslang bei dem vierten Patienten mit der Verdachtsdiagnose M. Crohn ein bidirektionaler endoskopischer Bergungsversuch. Unter der Klinik eines akuten Abdomens erfolgte bei diesem Patienten eine notfallmäßige Laparotomie mit Dünndarmteilresektion und Kapselbergung. Ursache des akuten Abdomens mit einer eitrigen Peritonitis war eine Striktur $150 \mathrm{~cm}$ vor der Bauhin'schen Klappe. Die Histologie des Dünndarmresektates ergab einen floriden M. Crohn mit Fistelung und Wandabszedierung aufgrund einer akuten ulzerierenden Ileitis.

Die Komplikationsrate dieser vier Patienten mit Kapselbergung durch Medikamente, Endoskopie und Operation lag bei 2\% (4/203). 
Abb. 17 illustriert den Zeitpunkt, zu dem die Kapsel die Ileozökalklappe jeweils passierte bzw. die einzelnen Bergungsmethoden bei fehlendem spontanen Kapselabgang.

Abb. 17: VKE-Passage durch die Ileozökalklappe

\begin{tabular}{|l}
$\square$ Passage $\leq 9$ Stunden \\
$\square$ Passage $>9$ Stunden \\
$\square$ Passage nach Darmlavage \\
Bergung durch endoskopische Intervention \\
Bergung durch chirurgische Intervention
\end{tabular}




\subsection{Diagnosen}

\subsubsection{Pathologische Befunde}

Bei 94 der 203 Patienten (46\%) wiesen alle drei Dünndarmsegmente einen pathologischen Befund auf, bei 79 Patienten (39\%) war zumindest eines der Segmente betroffen. Bei 22 Patienten (11\%) konnten hingegen keine Auffälligkeiten festgestellt werden. Für acht Patienten (4\%) liegen diesbezüglich keine Angaben vor, da entweder der Dünndarm zu stark verschmutzt war, sodass keine Auswertung erfolgen konnte oder die Kapsel den Dünndarm nicht erreicht hatte. Insgesamt zeigten 51 Patienten $(25,1 \%)$ des Patientenkollektivs einen verschmutzten Dünndarm. In Zusammenschau dieser Ergebnisse konnten bei $85,2 \%$ der Patientenpopulation (173/203) pathologische Auffälligkeiten festgestellt werden.

Die Einteilung pathologischer Befunde im Dünndarm erfolgt in drei Abschnitte, die sich aus je einem Drittel Passagezeit zwischen Pylorus und Bauhin'scher-Klappe ergeben. Die Verteilung der Resultate auf die jeweiligen Dünndarmdrittel ist fast gleichmäßig: das erste Drittel wies bei 135 Patienten (66,5\%) Veränderungen auf, das zweite Drittel bei 137 Patienten (67,5\%) und das dritte Drittel bei 136 Patienten (67\%). Die Anzahl der pathologischen Schleimhautveränderungen in den jeweiligen Dünndarmdritteln sowie die nummerische Aufteilung über die drei Dünndarmsegmente sind der nachstehenden Abb. 18 zu entnehmen.

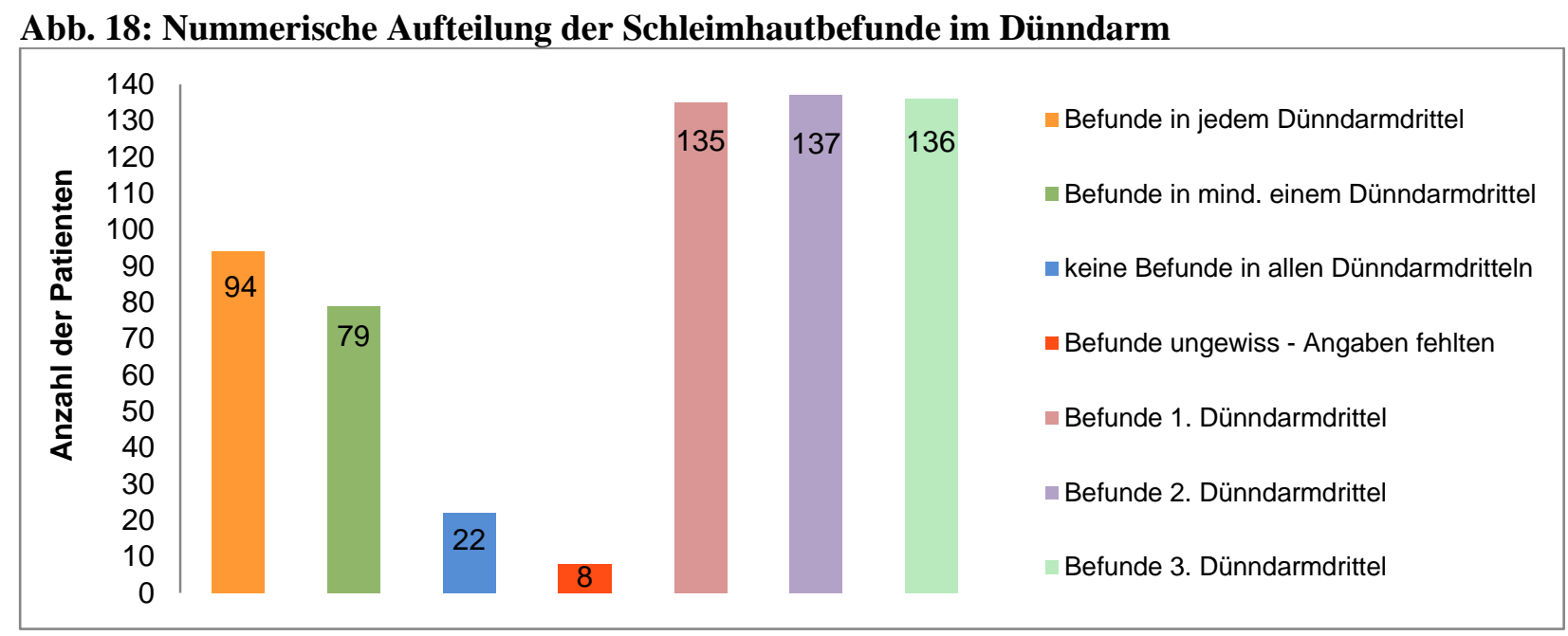


Die genauen pathologischen Veränderungen der 173 Patienten (85,2\%), welche entweder in jedem oder in mindestens einem Dünndarmsegment nachgewiesen werden konnten, sind in Tabelle 7 aufgeschlüsselt.

Tabelle 7: Pathologische VKE-Veränderungen

\begin{tabular}{|c|c|}
\hline Erhobene pathologische Befunde & Patienten $(n=173)$ \\
\hline Schleimhautläsionen (Erosionen, Ulzerationen) & $131(75,7 \%)$ \\
\hline kapilläre Läsionen (Angiodysplasien, Petechien) & $76(43,9 \%)$ \\
\hline $\begin{array}{l}\text { Schleimhautveränderungen (Erythem, Ödem, prominente } \\
\text { Schleimhautfalten) }\end{array}$ & $70 \quad(40,5 \%)$ \\
\hline Zottenrelief-Veränderungen (verplumpt, verquollen) & $72(41,6 \%)$ \\
\hline Venektasien & $28(16,2 \%)$ \\
\hline Lymphangiektasien/ lymphozytäre Infiltrate/ Lymphfollikel & $102(59 \%)$ \\
\hline $\begin{array}{l}\text { floride Dünndarmblutungen (Erosionen, Ulzerationen, } \\
\text { Angiodysplasien, Polypen) }\end{array}$ & $27(15,6 \%)$ \\
\hline Morbus Crohn-Läsionen (Aphten, Erythem, Ulzerationen) & $12(6,9 \%)$ \\
\hline Polypen & $30(17,3 \%)$ \\
\hline $\begin{array}{l}\text { Lumenveränderungen (extrinsiche Enge, submuköse und } \\
\text { extrinsische Erhabenheit) }\end{array}$ & $29(16,8 \%)$ \\
\hline luftgefüllte Dünndarmschlingen & $22(12,7 \%)$ \\
\hline gesteigerte Dünndarmperistaltik & $8(4,6 \%)$ \\
\hline Gastritis, Ulzerationen, portal hypertensive Gastropathie & $26(15 \%)$ \\
\hline $\begin{array}{l}\text { floride Kolonblutung (Angiodysplasien, Ulzerationen, } \\
\text { Teleangiektasien, Divertikel, iatrogen) }\end{array}$ & $7(4 \%)$ \\
\hline
\end{tabular}

Die folgenden VKE-Bilder (Abb. 19 bis Abb. 31) zeigen die oben erwähnten pathologischen Befunde des Magens und des Dünndarms im Vergleich zum normalen Schleimhautbefund. 
Abb. 19: Magen

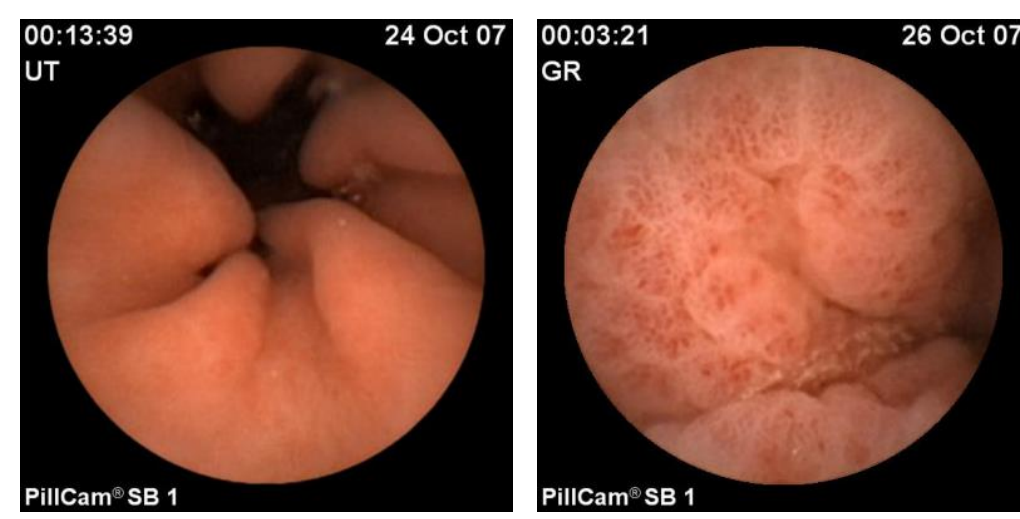

physiologische Schleimhaut

Antrumgastritis
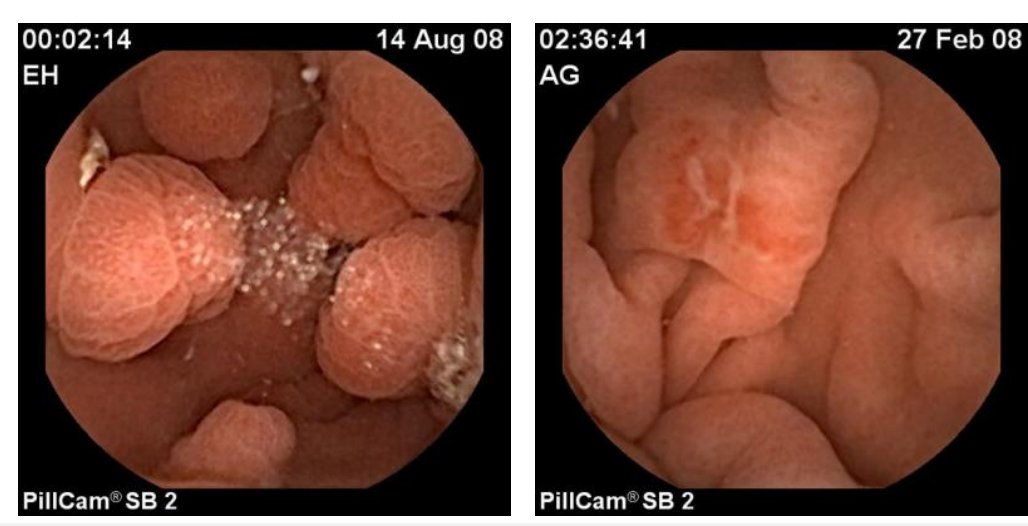

erhabene Falten im Antrum

Magenulcus

Abb. 20: Dünndarmbefunde

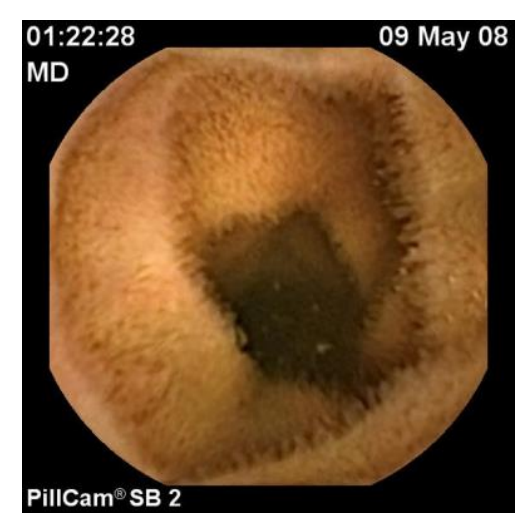

physiologische Schleimhaut mit typischer Zottenstruktur

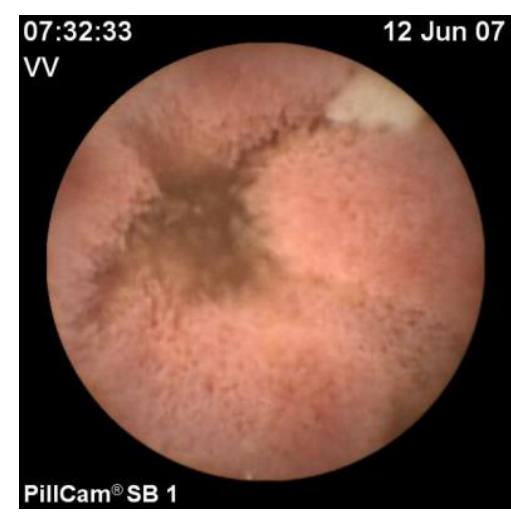

ödematöses Zottenrelief

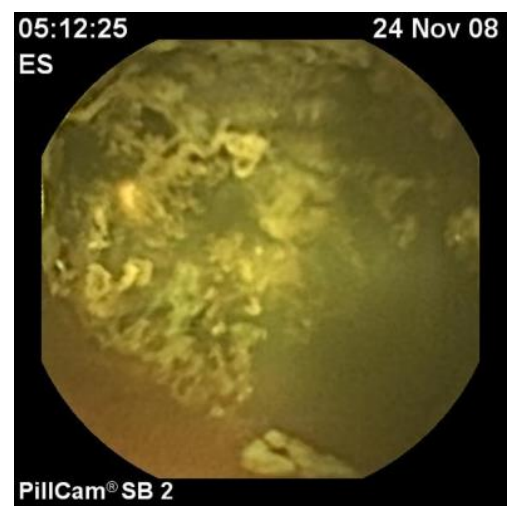

Verstuhlung 

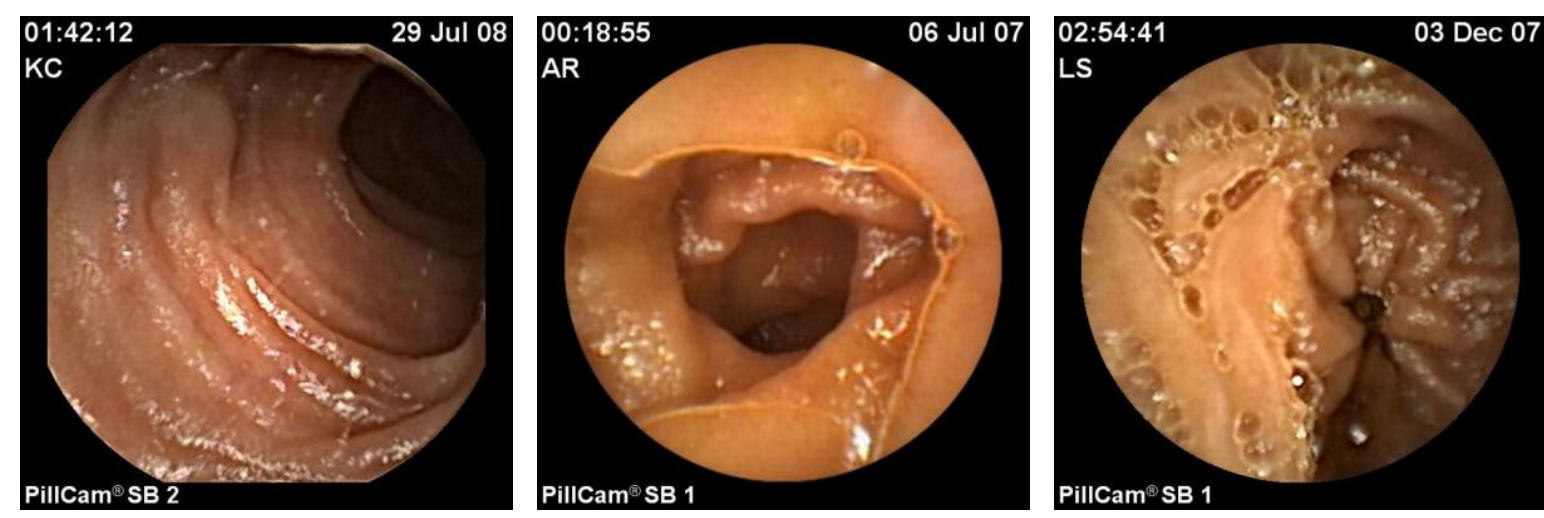

luftgefüllte Dünndarmschlingen

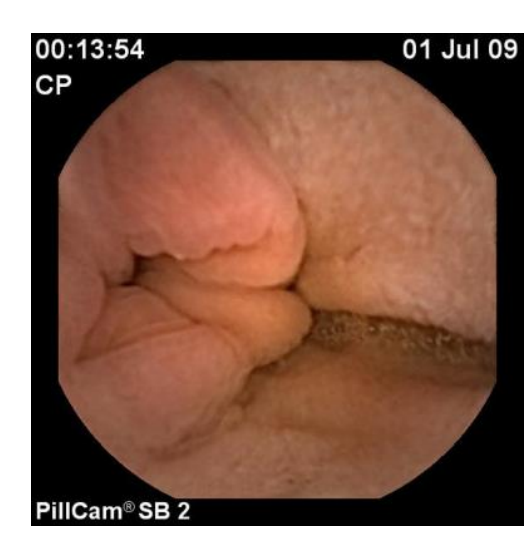

prominente Schleimhautfalten

Abb. 21: Pathologische Dünndarm-Läsionen

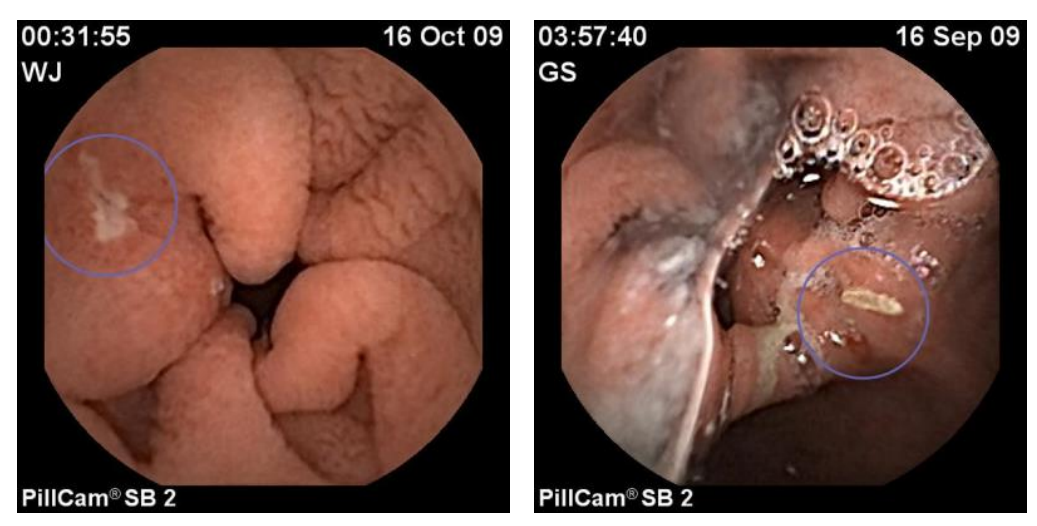




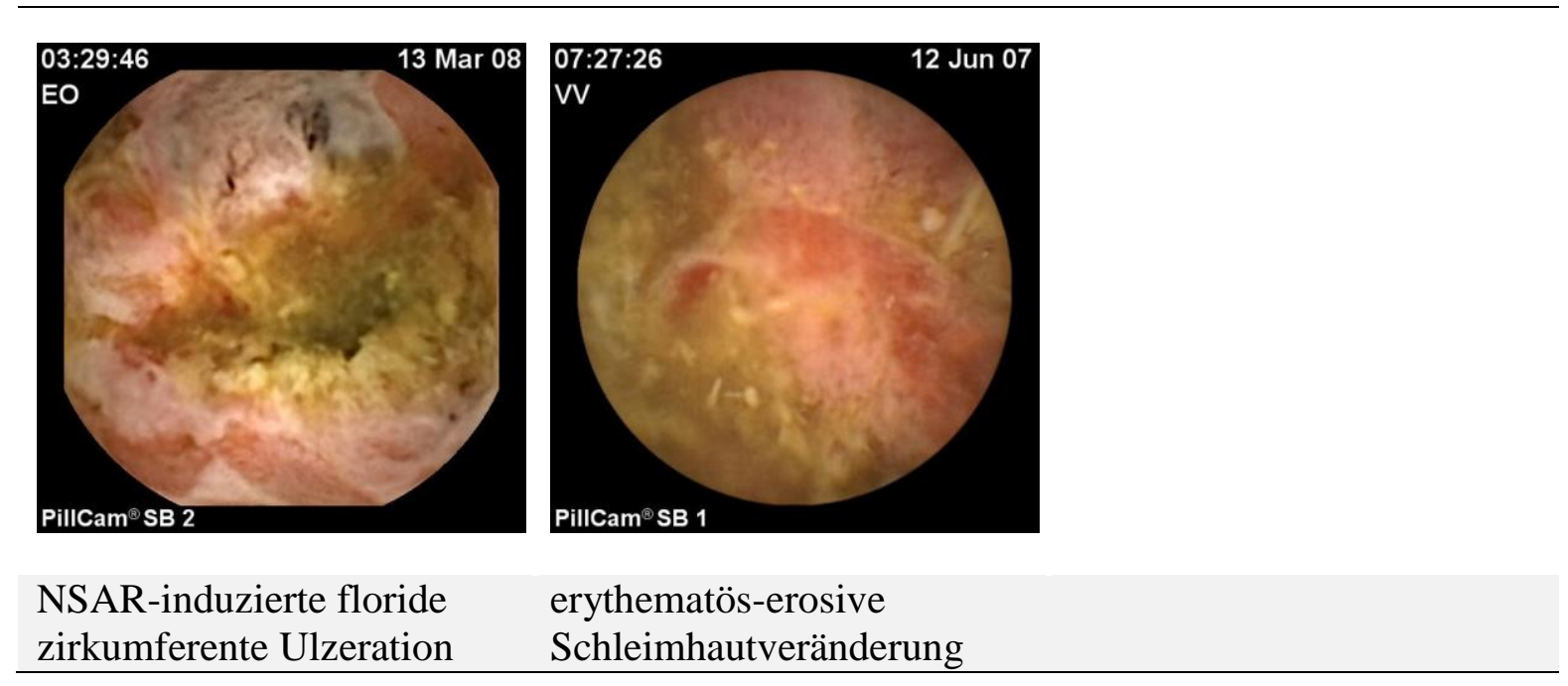

Abb. 22: Angiodysplasien

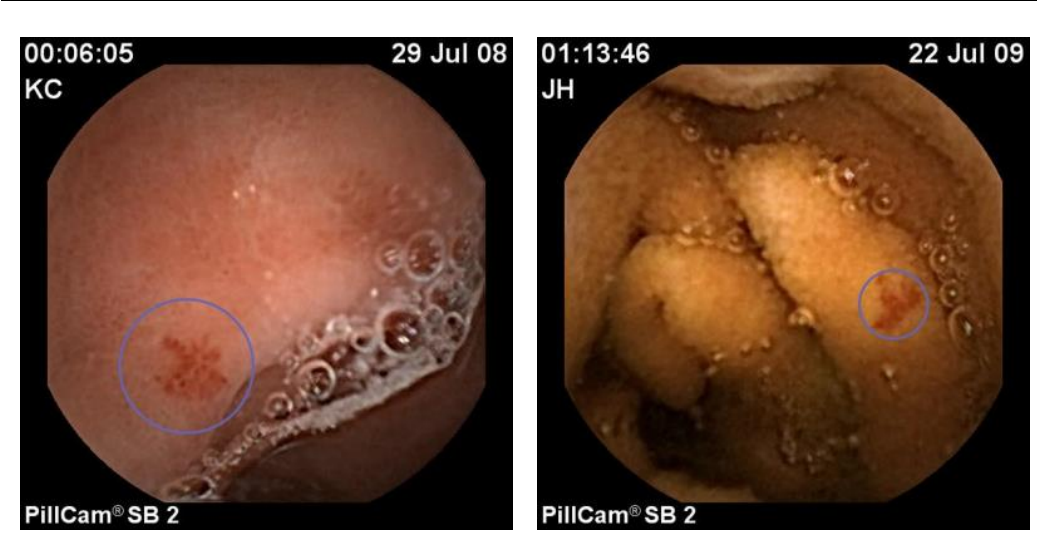

Abb. 23: Submuköse Erhabenheit mit Stenosierung

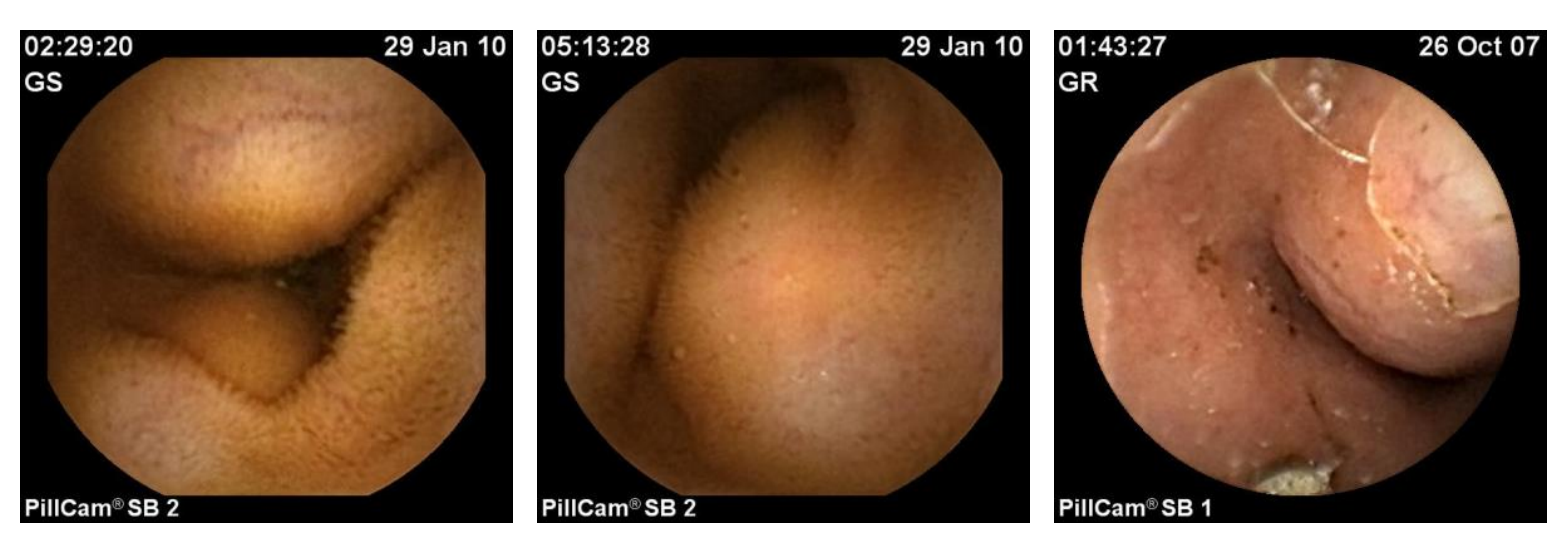


Abb. 24: Venektasien

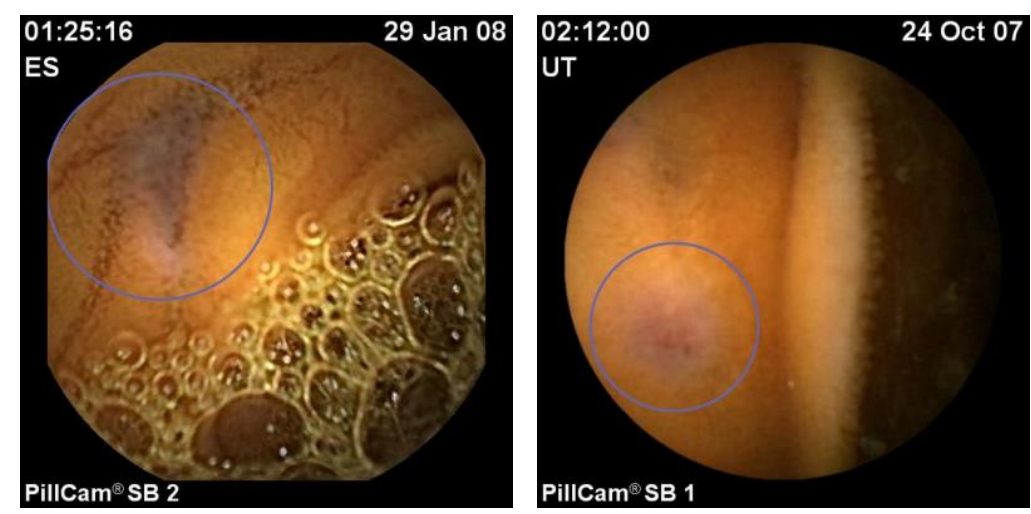

Abb. 25: Zystische Lymphangiektasien

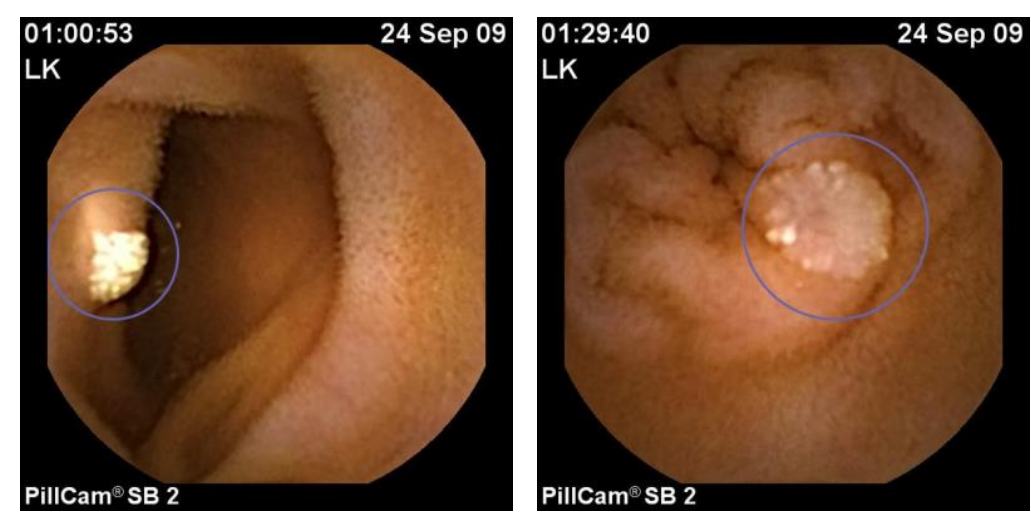

Abb. 26: Hypertrophierte Lymphfollikel
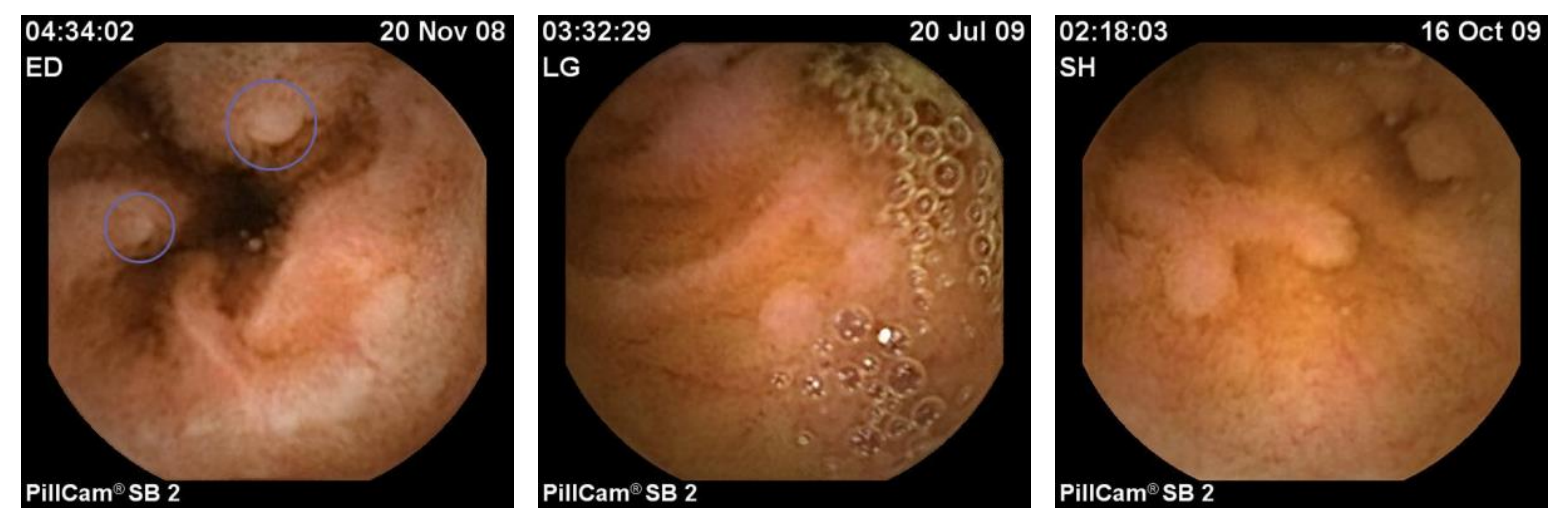

hypertrophierte Peyer

Plaques 
Abb. 27: Polypen/ Tumoren
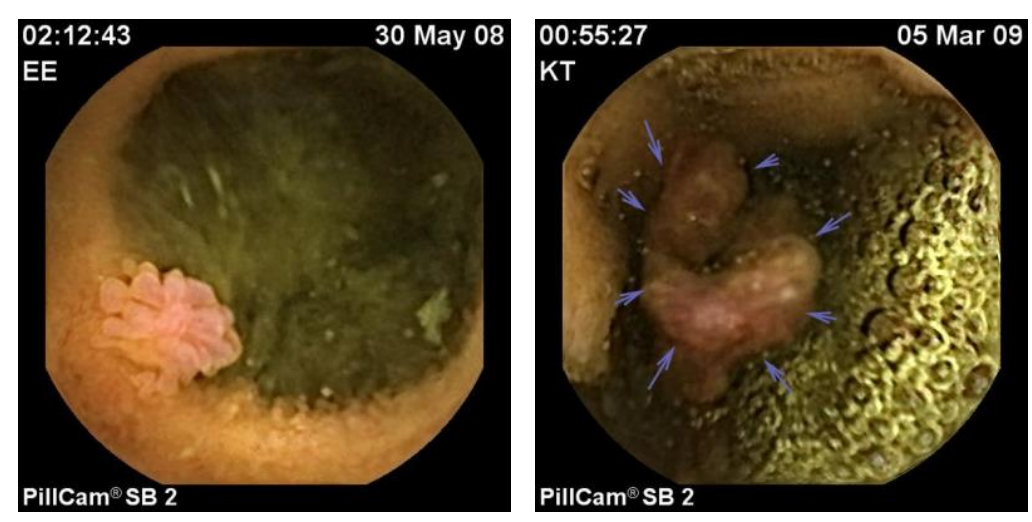

sessiler Polyp

Polyp unklarer Dignität

Abb. 28: Multiple Polypen bei FAP
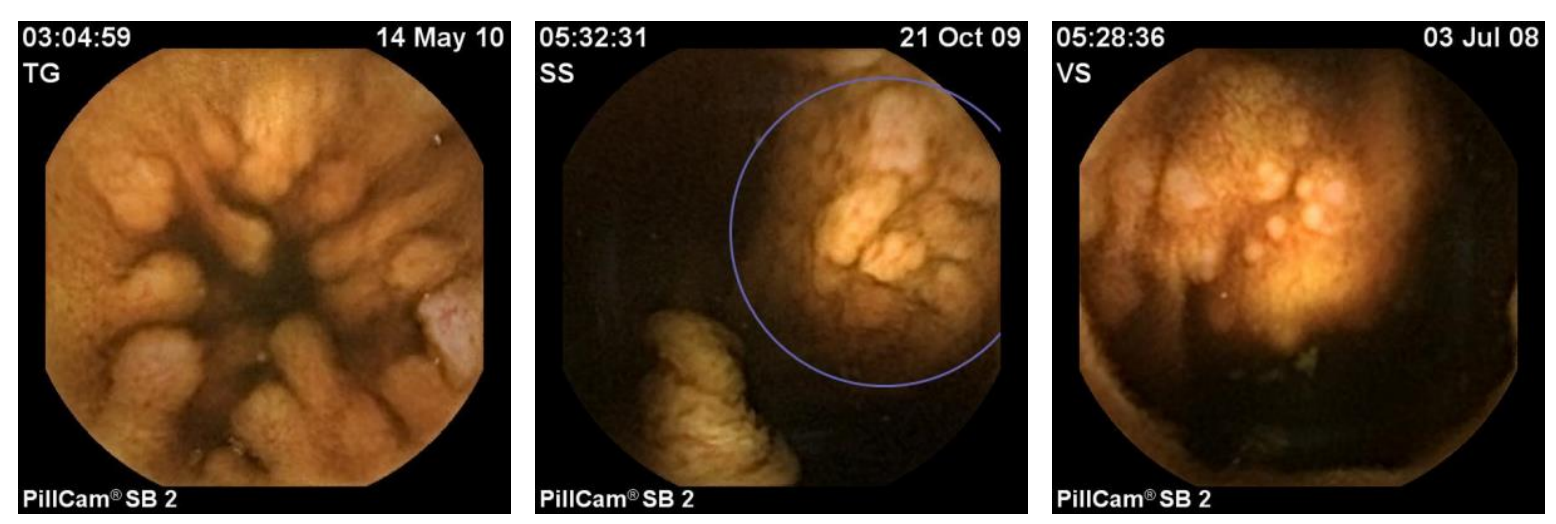

Abb. 29: Gesteigerte Dünndarmperistaltik
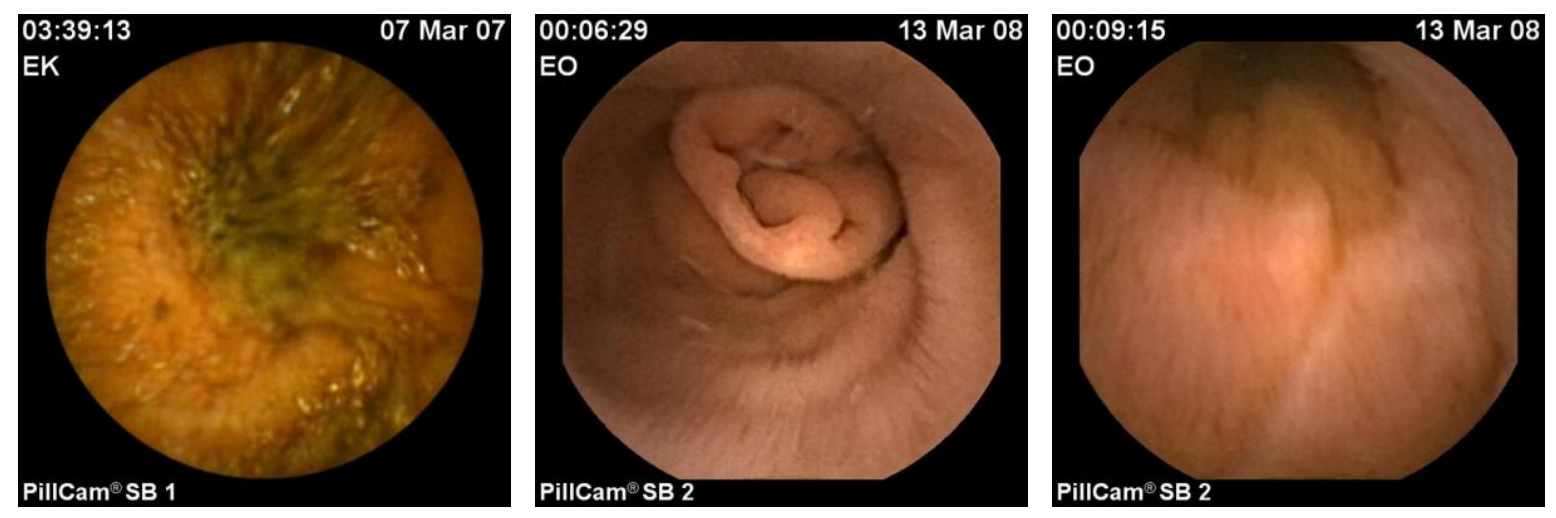
Abb. 30: Bauhin'sche Klappe

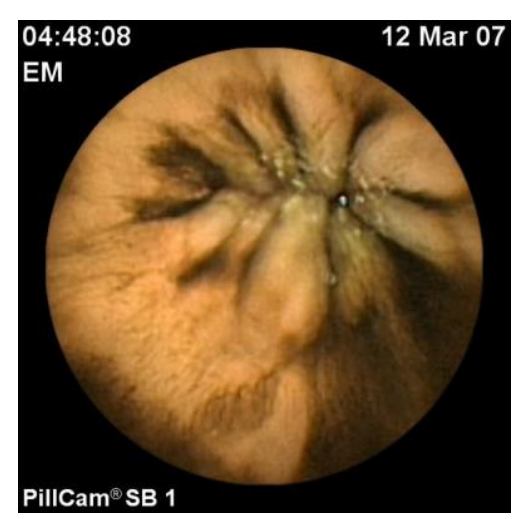

Abb. 31: Kuriose Befunde im Dünndarm

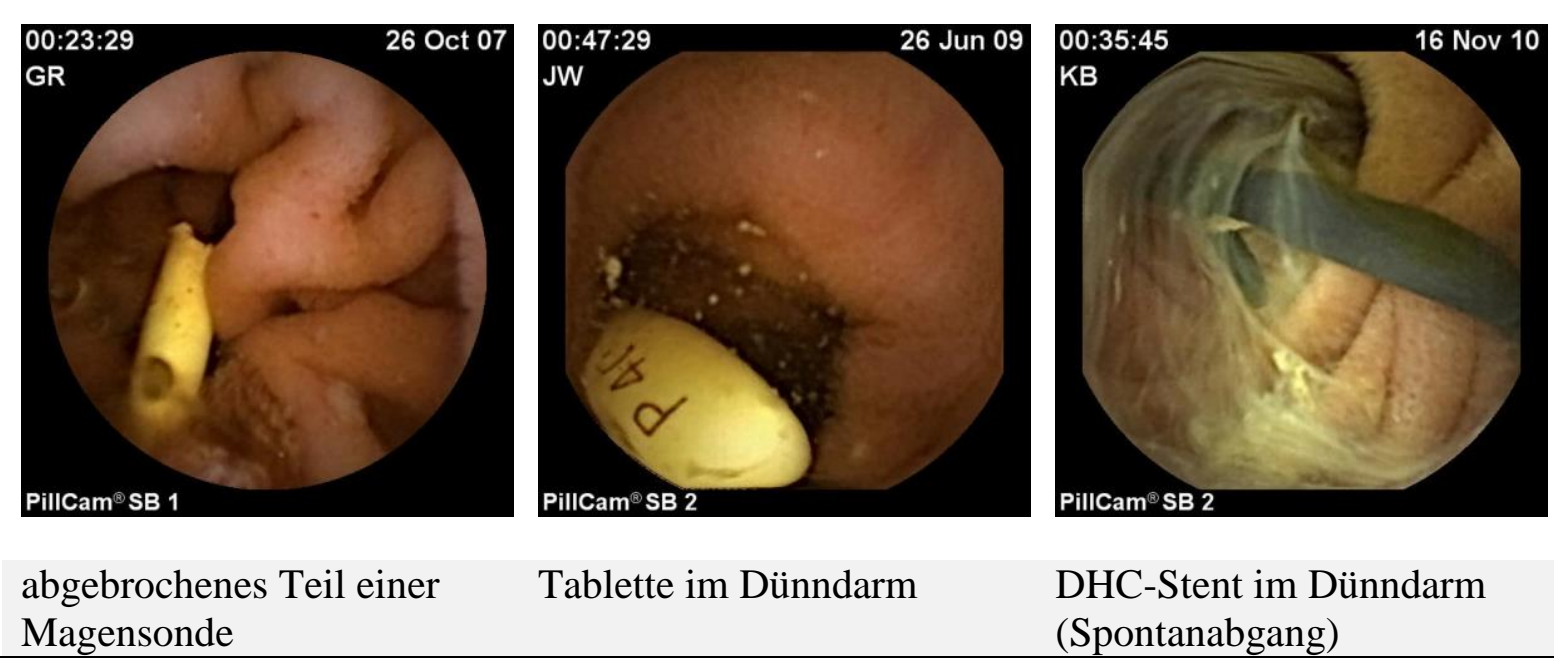

Die pathologischen Befunde der Indikationen: unklare gastrointestinale Blutung, unklare Anämie, unklare abdominelle Schmerzen, unklare Diarrhoe, rezidivierendes Erbrechen und Eiweißverlustsyndrom sind detailliert in den Tabellen 8 - 12 aufgeführt. 
Tabelle 8: Befunde der VKE-Indikation unklare gastrointestinale Blutung

\begin{tabular}{lc} 
Erhobene pathologische Befunde & Patienten $(\mathbf{n}=\mathbf{9 2})$ \\
$\begin{array}{l}\text { Schleimhautläsionen (Aphten, Erosionen, } \\
\text { Ulzerationen) }\end{array}$ & $44(47,8 \%)$ \\
$\begin{array}{l}\text { Befundkombinationen (Läsionen, Lymphangiek- } \\
\text { tasien, Angiodysplasien, tumoröse Veränderungen) }\end{array}$ & $27(29,3 \%)$ \\
$\begin{array}{l}\text { Gefäßveränderungen (Teleangiektasien, } \\
\text { Venektasien) }\end{array}$ & $3(3,3 \%)$ \\
$\begin{array}{l}\text { Normalbefund } \\
\text { kein Befundergebnis (Verstuhlung, keine Passage, } \\
\text { keine Blutungsquelle detektiert) }\end{array}$ & $10(10,9 \%)$ \\
diagnostische Ausbeute & $\mathbf{8 4}, 7 \%)$ \\
\hline
\end{tabular}

Tabelle 9: Befunde der VKE-Indikation unklare Anämie

\begin{tabular}{lc}
\hline Erhobene pathologische Befunde & Patienten $(\mathbf{n}=\mathbf{2 3})$ \\
\hline $\begin{array}{l}\text { Schleimhautläsionen (Ulzerationen) } \\
\text { Befundkombinationen (Erosionen, submuköse }\end{array}$ & $5(21,7 \%)$ \\
Erhabenheit, zystische Lymphangiektasien, & \\
Teleangiektasien) & \\
Gefäßveränderungen (Angiodysplasien, & $3(13 \%)$ \\
Lymphangiektasien, Venektasien) & $3(13 \%)$ \\
Normalbefund & $1(4,3 \%)$ \\
kein Befundergebnis (keine Aussage möglich) & $\mathbf{1 8 ( 7 8 \% )}$ \\
diagnostische Ausbeute & \\
\hline
\end{tabular}


Tabelle 10: Befunde der VKE-Indikation unklare abdominelle Schmerzen

Erhobene pathologische Befunde

Patienten $(n=49)$

Schleimhautläsionen (Aphten, Erosionen,

Ulzerationen)

$4(8 \%)$

Befundkombinationen (Aphten, Divertikel,

Erythem, Läsionen, Polyknospen, Stenosen)

$10(20,4 \%)$

Gefäßveränderungen (Angiodysplasien,

Lymphangiektasien, Venektasien)

$6(12,2 \%)$

Normalbefund

0

kein Befundergebnis (Verstuhlung, fehlende

oder zu lange Passage, keine Aussage)

$29(59,2 \%)$

diagnostische Ausbeute

$20(40,8 \%)$

Tabelle 11: Befunde der VKE-Indikation unklare Diarrhoe

\begin{tabular}{ll} 
Erhobene pathologische Befunde & Patienten $(\mathbf{n = 1 3})$ \\
\hline $\begin{array}{l}\text { Schleimhautläsionen (Erosionen, Ulzerationen) } \\
\text { Befundkombinationen (Apthen, Läsionen, }\end{array}$ & $1(7,7 \%)$ \\
Angiektasien, Lymphangiektasien, Zottenatrophie) & $5(38,5 \%)$ \\
Normalbefund & $7(53,8 \%)$ \\
kein Befundergebnis & 0 \\
diagnostische Ausbeute & $\mathbf{6 ( 4 6 \% )}$ \\
\hline
\end{tabular}

Tabelle 12: Befunde der VKE-Indikation rezidivierendes Erbrechen und Eiweißverlustsyndrom

\begin{tabular}{ll}
\hline Erhobene pathologische Befunde & Patienten \\
\hline Unklares rezidivierendes Erbechen: & \\
weißlich verdickte Dünndarmvilli: Zustand nach & $1(50 \%)$ \\
Duodenitis &
\end{tabular}


Eiweißverlustsyndrom:

ödematöse Dünndarmvilli: primär intestinale

$1(50 \%)$

Lymphangiektasie (Morbus Waldmann) 


\subsubsection{Dünndarmblutung}

Durch die Videokapselendoskopie konnte bei 27 Patienten (13,3\%; 10 Frauen und 17 Männer) eine floride Dünndarmblutung detektiert werden, nur bei 20 Patienten konnte eine Ursache gefunden werden, siehe Tabelle 13.

Von den 111 über 60-jährigen Patienten (54,7\%) waren 18 Patienten (16,2\%) von einer Blutung im Dünndarm betroffen $(\mathrm{p}=0,37)$. Bilder von Dünndarmblutungen sind in den Abb. 32 und 33 zu erkennen.

Abb. 32: floride Dünndarmblutungen ohne erkennbare Blutungsquelle

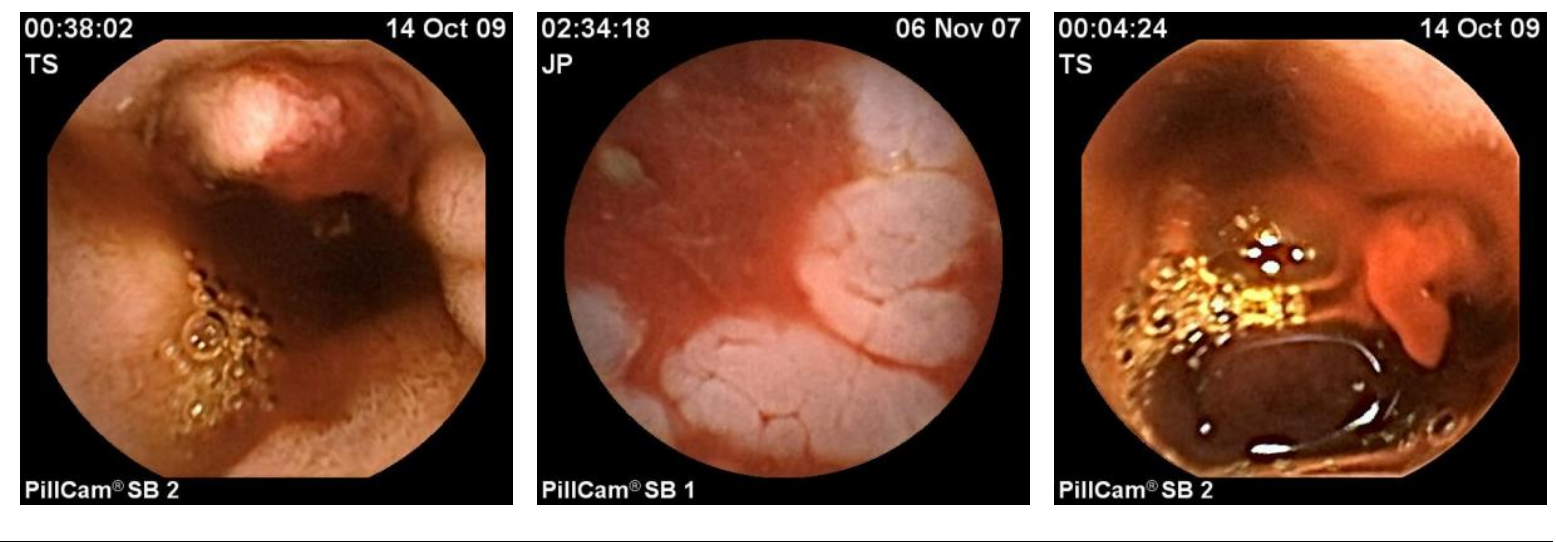

\section{Abb. 33: Teerstuhl im Dünndarm}

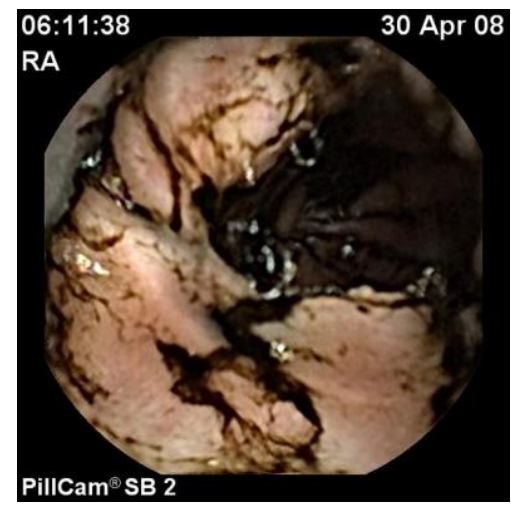


Tabelle 13: VKE-Befunde der Patienten mit floriden Dünndarmblutungen

\begin{tabular}{ll}
\hline Erhobene pathologische Befunde & Patienten $(\mathbf{n}=\mathbf{2 7})$ \\
\hline Ulzerationen & $9(33,3 \%)$ \\
Erosionen & $7(26 \%)$ \\
Angiodysplasien & $1(3,7 \%)$ \\
Divertikel & $1(3,7 \%)$ \\
polypoide Läsion & $1(3,7 \%)$ \\
iatrogen (Biopsie) & $1(3,7 \%)$ \\
kein Befundergebnis & $7(26 \%)$ \\
Diagnostische Ausbeute & $\mathbf{2 0}(\mathbf{7 4 \%})$ \\
\hline
\end{tabular}

Die Häufigkeitsverteilung der Patienten mit Dünndarmblutungen - abhängig von den verschiedenen VKE-Indikationen - ist aus Tabelle 14 ersichtlich. Von allen Patienten mit Dünndarmblutungen gehörten die meisten zur Indikationsgruppe der unklaren gastrointestinalen Blutung.

Tabelle 14: Häufigkeitsverteilung der Dünndarmblutungspatienten bei den verschiedenen VKE-Indikationen

\begin{tabular}{ll}
\hline Indikationen & Patienten $(\mathbf{n}=\mathbf{2 7})$ \\
\hline unklare gastrointestinale Blutungen & $18(66,7 \%)$ \\
\hline unklare Anämie & $1(3,7 \%)$ \\
unklare abdominelle Schmerzen & $2(7,4 \%)$ \\
Verdacht auf Morbus Crohn & $1(3,7 \%)$ \\
Komplikation bei Morbus Crohn & $2(7,4 \%)$ \\
unklare Diarrhoe & 0 \\
Polyp- und Tumorsuche & $2(7,4 \%)$ \\
unklares rezidivierendes Erbrechen & 0 \\
Eiweißverlustsyndrom & $1(3,7 \%)$ \\
\hline
\end{tabular}


Viele unserer Dünndarmblutungspatienten hatten bedeutsame Nebenerkrankungen. 12 der 27 Patienten wurden im Bereich des Magen-Darm-Traktes voroperiert. 10 Patienten nahmen Medikamente ein, die die Blutgerinnung beeinflussen. Die einzelnen anamnestischen Daten sind aus Tabelle 15 zu entnehmen.

Tabelle 15: Anamnestische Angaben der gastrointestinalen Blutungspatienten

\begin{tabular}{|c|c|}
\hline Patienten-Anamnese & Patienten $(n=27)$ \\
\hline $\begin{array}{l}\text { Erkrankungen im Magen-Darm-Trakt }(\mathrm{n}=48) \\
\text { (Cholezystitis, Leberzirrhose, } \\
\text { Steatosis hepatis) }\end{array}$ & $5(18,5 \%)$ \\
\hline Diabetes mellitus $(\mathrm{n}=29)$ & $4(14,8 \%)$ \\
\hline $\begin{array}{l}\text { Operationen im Magen-Darm-Trakt }(\mathrm{n}=77 \text { ) } \\
\text { (Appendektomien, Bridenlösung, } \\
\text { Cholezystektomien, diagnostische } \\
\text { Laparostomie, Dünndarmteilresektion, } \\
\text { Herniotomie, Leberteilresektion, } \\
\text { Lebertransplantation, Magenteilresektion, } \\
\text { partielle oder totale Kolektomien) }\end{array}$ & $12(44,4 \%)$ \\
\hline $\begin{array}{l}\text { Medikamente der Antikoagulation }(\mathrm{n}=72) \\
\text { (Acetylsalicylsäure, Clopidogrel, Phenprocoumon) }\end{array}$ & $10(37 \%)$ \\
\hline $\begin{array}{l}\text { Magenschleimhautschädigende Medikamente } \\
(\mathrm{n}=32) \text { (NSAR, Steroide, Immunsuppressiva) }\end{array}$ & $6(22,2 \%)$ \\
\hline Zigaretten-Abusus $(n=24)$ & $1(3,7 \%)$ \\
\hline
\end{tabular}

Die Mehrzahl unserer Studienpatienten (19/27) mit floriden Dünndarmblutungen gehörten zu der BMI-Gruppe II und III (normal- und übergewichtig), siehe Tabelle 16. 
Tabelle 16: Dünndarmblutungen bei den unterschiedlichen BMI-Kategorien, siehe S. 38

\begin{tabular}{lcll}
\hline BMI & Patienten $(\mathbf{n}=\mathbf{2 7})$ & Frauen & Männer \\
Gruppe I $\quad(\mathrm{n}=15)$ & $4(14,8 \%)$ & 1 & 3 \\
Gruppe II $\quad(\mathrm{n}=75)$ & $11(40,8 \%)$ & 4 & 7 \\
Gruppe III $(\mathrm{n}=85)$ & $8(29,6 \%)$ & 2 & 6 \\
Unbekannt $(\mathrm{n}=28)$ & $4(14,8 \%)$ & 3 & 1 \\
\hline
\end{tabular}

Die Magenpassagezeiten der 27 Patienten mit Dünndarmblutungen lagen zwischen zwei Minuten und 462 Minuten ( $\overline{\mathrm{x}}=18$ Minuten). Die Dünndarmpassagezeiten erreichten ein Minimum von 136 Minuten und ein Maximum von 540 Minuten $(\overline{\mathrm{x}}=327$ Minuten $)$.

Bei 24 Patienten dieser Gruppe konnte der Hämoglobinwert aus der Patientenakte eruiert werden, der minimale Wert lag bei 3,7 g/dl und der maximale Wert bei 15,3 g/dl. Der durchschnittliche Wert betrug 9,3 g/dl $( \pm 2,96)$.

Von den 203 Patienten hatten 60 (29,6\%) zusätzlich die Diagnose Anämie, der durchschnittliche Hämoglobinwert befand sich bei 7,6 g/dl. 


\subsubsection{Morbus Crohn}

Die Videokapselendoskopie konnte bei zwölf Patienten (6\%; 2 Frauen und 10 Männer) des Patientenkollektivs typische Läsionen eines Morbus Crohn nachweisen. Das Durchschnittsalter dieser Patienten lag bei 42 Jahren (16 bis 77 Jahre). Sechs Patienten (50\%) waren unter 30 Jahre alt, vier Patienten (33\%) zwischen 30-60 Jahre alt und zwei Patienten (17\%) waren älter als 60 Jahre.

Der BMI der M. Crohn-Patienten entsprach durchschnittlich 22,25 (BMI 17 bis 29). Drei Patienten (25\%) gehörten der BMI-Gruppe I an, sieben Patienten (58\%) der BMI-Gruppe II und die restlichen zwei Patienten (17\%) der BMI-Gruppe III.

Die Dauer des stationären Krankenhausaufenthaltes lag bis auf einen dieser Patienten, der ambulant behandelt wurde bei 8,4 Tagen ( 5 bis 11 Tage).

Bei fünf Patienten (2,5\%; zwei Frauen und drei Männer) bestand die Diagnose M. Crohn vor der VKE. Einer dieser fünf Patienten zeigte eine unauffällige Dünndarmschleimhaut. Typische Veränderungen wie aphthöse Läsionen und fibrinbelegte Ulzerationen wurden bei den anderen vier Patienten befundet. Zwei Patienten wiesen davon eine Dünndarmblutung auf. Drei der fünf Patienten (60\%) erhielten wegen Blutung und/ oder Anämie EK-Einheiten und ein Patient $(16,7 \%)$ ein FFP-Konzentrat.

Ein Verdacht auf M. Crohn war bei acht Patienten (4\%; zwei Frauen und sechs Männer) ausschlaggebend für die VKE. Bei fünf $(62,5 \%)$ dieser acht Patienten konnte die Diagnose Morbus Crohn anhand der Videokapselendoskopie gestellt werden. Davon wies ein Patient eine Dünndarmblutung auf. Bei den restlichen drei Patienten (37,5\%) konnte der Verdacht weder bestätigt noch ausgeschlossen werden. Bei einem Patienten (12,5\%) wurde die Indikation aufgrund von unauffälligen Schleimhautverhältnissen im Dünndarm ausgeschlossen. Ein anderer Patient $(12,5 \%)$ zeigte einige fibrinbelegte Ulzerationen, jedoch erschien der restliche Dünndarm unauffällig. Bei dem dritten Patient (12,5\%) war eine diagnostische Aussage, aufgrund von Passagestörungen über die gesamte Aufnahmezeit, bei Verbleiben der Kapsel im Magen nicht möglich. Niemand aus der Gruppe mit Verdacht auf M. Crohn erhielt ein EKoder FFP-Präparat.

Von den anderen drei Patienten, bei denen die Diagnose M. Crohn durch die VKE gestellt werden konnte, hatten zwei Patienten die Indikation unklare abdominelle Schmerzen sowie ein Patient die Indikation unklare gastrointestinale Blutung. 
Die Magenpassagezeit war bei allen zwölf Patienten, bei denen M. Crohn-typische Schleimhautveränderungen gesehen werden konnten, nicht verlängert. Die Passagezeit lag unter 100 Minuten, im Durchschnitt bei 28,7 Minuten (zwischen zwei bis 66 Minuten). Die durchschnittliche Dünndarmtransitzeit lag bei den zwölf Patienten bei 360,3 Minuten (zwischen 142 und dem Maximum 540 Minuten). Die Passagezeit des Dünndarms war bei vier Patienten über 350 Minuten verlängert.

Abb. 34 und Abb. 35 zeigen Schleimhautveränderungen der M. Crohn-Patienten.

Abb. 34: M. Crohn Veränderungen I
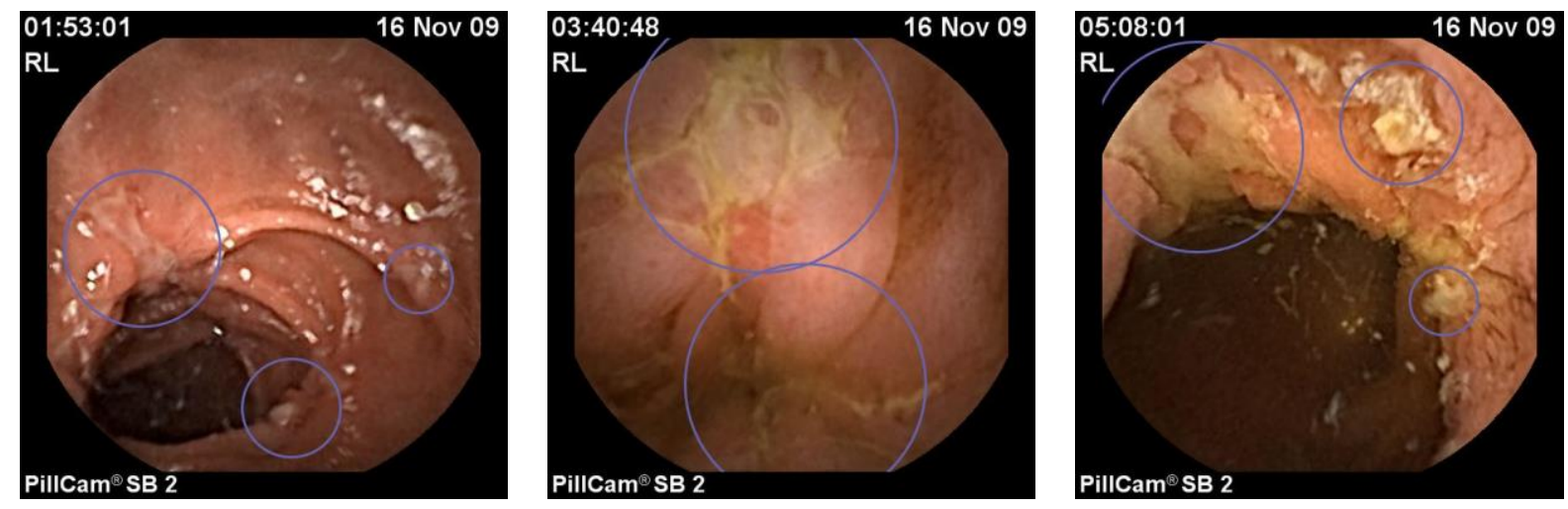

fibrinbelegte Ulzerationen

Abb. 35: M. Crohn Veränderungen II

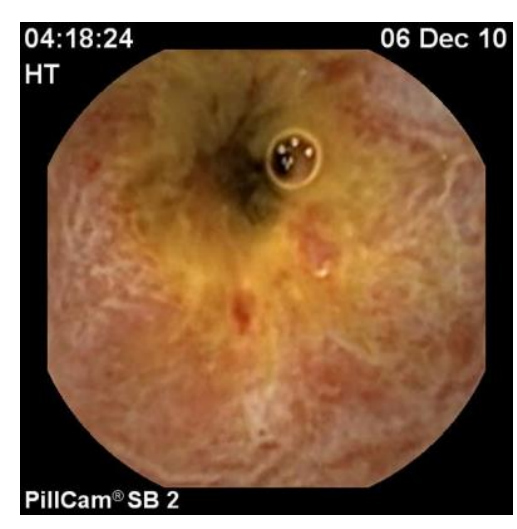

zirkuläre fibrinbelegte Ulzeration mit subtotaler Stenosierung

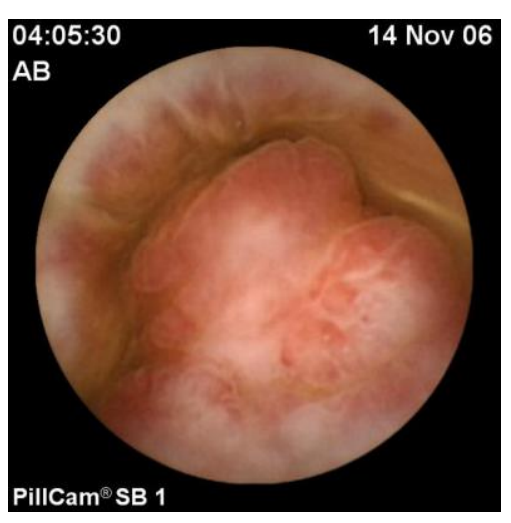

entzündliche Veränderung der Ileozökalklappe 
In der Videokapselendoskopie waren folgende typische Veränderungen bei den zwölf Morbus Crohn-Patienten sichtbar: Schleimhautläsionen bei zehn Patienten (83,3\%), davon aphthöse Läsionen bei sechs Patienten (50\%), Erosionen bei sieben Patienten (58,3\%) und fibrinbelegte Ulzerationen bei acht Patienten (66,7\%). Ein Erythem, gerötete Schleimhaut, war bei sechs Patienten (50\%), ein abgeflachtes und verplumptes Zottenrelief bei sechs Patienten (50\%) sowie zystische Lymphangiektasien bei fünf Patienten $(41,7 \%)$ vorhanden.

Folgende atypische Kriterien konnten bei den Morbus Crohn-Patienten gefunden werden: Lumenveränderungen bei vier Patienten (33,3\%), extrinsische Enge bei drei Patienten (25\%), submuköse Erhabenheit bei einem Patient (8,3\%), Angiodysplasien bei vier Patienten (33,3\%) und venöse Läsionen bei einem Patient (8,3\%).

Das erste Dünndarmdrittel zeigte bei acht diagnostizierten M. Crohn-Patienten (66,7\%) Schleimhautaufälligkeiten. Die Schleimhaut der zweiten und dritten Dünndarmsegmente war bei allen zwölf Patienten verändert.

Tabelle 17 zeigt eine Übersicht der pathologischen Schleimhautveränderungen der Patienten mit der Indikation Komplikation bei M. Crohn sowie dem Verdacht auf M. Crohn und der durch die VKE diagnostizierten M. Crohn-Patienten.

Tabelle 17: Dünndarmveränderungen der Morbus Crohn- und V.a. M. Crohn-Patienten

\begin{tabular}{|c|c|c|c|}
\hline $\begin{array}{l}\text { Erhobene pathologische } \\
\text { Befunde: }\end{array}$ & $\begin{array}{l}\text { Indikation: } \\
\text { Komplikation bei } \\
\text { M. Crohn }(\mathrm{n}=\mathbf{5})\end{array}$ & $\begin{array}{l}\text { Indikation: } \\
\text { V.a. M. Crohn } \\
(\mathbf{n}=\mathbf{8})\end{array}$ & $\begin{array}{l}\text { Diagnosestellung } \\
\text { M. Crohn durch } \\
\text { VKE }(\mathrm{n}=12)\end{array}$ \\
\hline $\begin{array}{l}\text { Schleimhautläsionen } \\
\text { (Erosion, Ulzeration) }\end{array}$ & $3(60 \%)$ & $6(75 \%)$ & $10(83,3 \%)$ \\
\hline $\begin{array}{l}\text { Schleimhaut- } \\
\text { veränderungen (Rötung, } \\
\text { Schleimhautfalte, } \\
\text { verplumptes Zottenrelief) }\end{array}$ & $3(60 \%)$ & $5(62,5 \%)$ & $7(\%)$ \\
\hline $\begin{array}{l}\text { zystische } \\
\text { Lymphangiektasien }\end{array}$ & $1(20 \%)$ & $4(50 \%)$ & $5(41,7 \%)$ \\
\hline $\begin{array}{l}\text { Angiodysplasien } \\
\text { (kapilläre Läsionen) }\end{array}$ & $1(20 \%)$ & $2(25 \%)$ & $4(33,3 \%)$ \\
\hline Venektasien & ---------- & $1(12,5 \%)$ & $1(8,3 \%)$ \\
\hline
\end{tabular}




$\begin{array}{llll}\begin{array}{l}\text { Lumenveränderungen } \\ \text { (submuköse und } \\ \text { extrinsische }\end{array} & 3(37,5 \%) & 4(33,3 \%) \\ \begin{array}{l}\text { Erhabenheiten, } \\ \text { extrinsische Enge) }\end{array} & 1(20 \%) & 1(12,5 \%) & 2(16,7 \%) \\ \text { Polypen } & 2(40 \%) & 1(12,5 \%) & 3(25 \%) \\ \begin{array}{l}\text { Dünndarmblutung } \\ \text { Lokalisation }\end{array} & 2(40 \%) & 5(62,5 \%) & 8(66,7 \%) \\ \begin{array}{l}1 . \text { Dünndarmdrittel } \\ \text { Lokalisation }\end{array} & 4(80 \%) & 7(87,5 \%) & 12(100 \%) \\ \text { 2.+ 3. Dünndarmdrittel } & & & \\ \text { Diagnostische Ausbeute } & \mathbf{4 ( 8 0 \% )} & \mathbf{5 ( 6 2 , 5 \% )} & \end{array}$

Sechs Videokapseln (50\%) wurden von den zwölf gesicherten M. Crohn-Patienten im Rahmen des stationären Aufenthaltes via naturalis ausgeschieden. Bei drei Patienten (25\%) erfolgte eine verzögerte Ausscheidung nach mehreren Wochen, kontrolliert durch eine Detektion der Kapsel mit Hilfe einer Röntgen-Abdomen-Übersicht. Bei den drei anderen M. Crohn-Patienten (25\%) wurde eine endoskopische Bergung der Videokapsel versucht, die in einem Fall $(8,3 \%)$ erfolgreich verlief. Bei einem anderen Patienten (8,3\%) konnte die Videokapsel nach einer Darmlavage geborgen werden. Bei dem dritten Patienten $(8,3 \%)$ war eine chirurgische Bergung, aufgrund einer Stenosierung des Ileums, durch eine Dünndarmsegmentresektion notwendig. 


\subsubsection{Dünndarmtumoren}

Ausgehend von den 203 Patienten konnten bei 37 Patienten (18,2\%) Tumoren unterschiedlicher Entität aus der Anamnese erhoben werden. In Abb. 36 sind die verschiedenen Tumoren des Patientenkollektivs aufgeführt.

\section{Abb. 36: Unterschiedliche Tumoren des Patientenkollektivs}

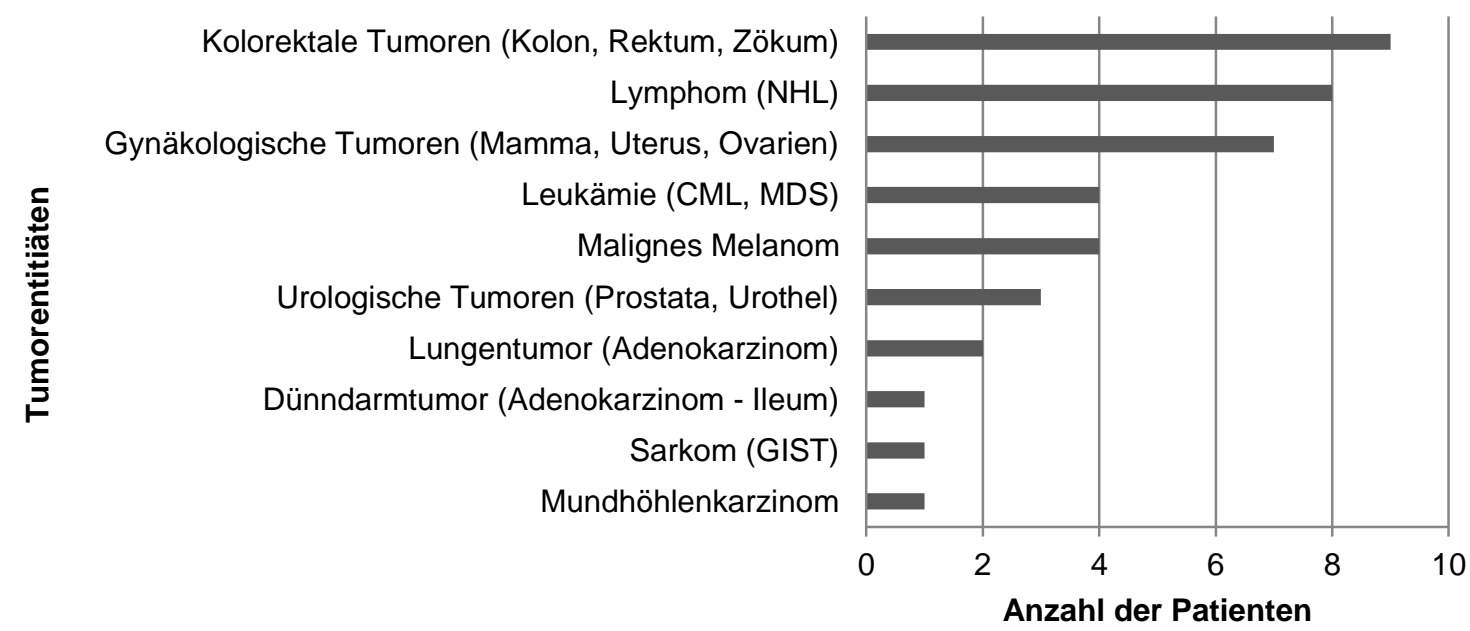

Die Indikation Polyp- und Tumorsuche war bei elf Patienten (5,4\%) für die VKE maßgebend. Tabelle 18 veranschaulicht, welche Diagnosen bei diesen Patienten gestellt wurden. 
Tabelle 18: Befunde der VKE-Indikation Polyp- und Tumorsuche

\begin{tabular}{|c|c|c|}
\hline Anamnese & $\begin{array}{l}\text { Erhobene pathologische } \\
\text { Befunde: }\end{array}$ & Patienten $(n=11)$ \\
\hline $\begin{array}{l}\text { Kontrolle nach tubulärem } \\
\text { Rektum-Karzinom, Colitis: }\end{array}$ & kein Polyp, flache Läsion & $1(9 \%)$ \\
\hline $\begin{array}{l}\text { Kontrolle nach Lymphom-Befall } \\
\text { (vgl. Tabelle } 19 \text { - Fall 1): }\end{array}$ & $\begin{array}{l}\text { lymphozytäre Infiltration, } \\
\text { zystische Lymphangiektasien } \\
\text { (Lymphombefall möglich) }\end{array}$ & $1(9 \%)$ \\
\hline $\begin{array}{l}\text { Kontrolle nach Dünndarm-GIST } \\
\text { (vgl. Tabelle } 20 \text { - Fall 2): }\end{array}$ & $\begin{array}{l}\text { vergröbertes Zottenrelief, } \\
\text { Erosionen, } \\
\text { DD: erosive Antrumgastritis }\end{array}$ & $1(9 \%)$ \\
\hline Kontrolle nach NHL: & lymphozytäre Infiltration & $1(9 \%)$ \\
\hline Kontrolle bei FAP: & $\begin{array}{l}\text { multiple Polypenknospen, } \\
\text { kein Tumor }\end{array}$ & $4(37 \%)$ \\
\hline Kontrolle bei FAP: & $\begin{array}{l}\text { multiple Polypenknospen, } \\
\text { Erosion mit Blutspur, } \\
\text { kein Tumor }\end{array}$ & $1(9 \%)$ \\
\hline $\begin{array}{l}\text { Kontrolle eines Ileumpolypen, } \\
\text { nach Röntgen-Sellink: }\end{array}$ & $\begin{array}{l}\text { exulzerierender Polyp / } \\
\text { DD: Adenom / } \\
\text { DD: Karzinom, multiple } \\
\text { Polypenknospen }\end{array}$ & $1(9 \%)$ \\
\hline $\begin{array}{l}\text { Kontrolle nach Adenomresektion } \\
\text { im Ileum: }\end{array}$ & fibrinbelegte Ulzeration & $1(9 \%)$ \\
\hline diagnostische Ausbeute & & $10(90,9 \%)$ \\
\hline
\end{tabular}

In den Voruntersuchungen konnte bei vier Patienten (2\%; eine Frau und drei Männer) eine tumorverdächtige Veränderung im Dünndarm nachgewiesen werden. Ein Patient zeigte während einer ÖGD Auffälligkeiten im oberen Dünndarm, zwei Patienten im Rahmen einer BallonEnteroskopie und der vierte Patient bei einer computertomografischen Untersuchung des Abdomens.

Das Alter dieser Patienten lag zwischen 40 und 65 Jahren $(\overline{\mathrm{x}}=52$ Jahre $)$. Bei zwei Patienten bestand die Indikation Polyp- und Tumor-Suche bei unspezifischen abdominellen Beschwerden oder ungewollten Gewichtsverlustes von $4 \mathrm{~kg}$ in vier Wochen. Die anderen beiden Patienten 
wurden wegen einer unklaren Hämoglobin-relevanten gastrointestinalen Blutung auffällig. Die beiden letztgenannten Patienten zeigten einen positiven Hämoccult-Test.

Zur Tumorlokalisation und Ausschluss/ Nachweis einer weiteren Tumormanifestation wurde bei diesen Patienten die VKE durchgeführt.

Die kapselendoskopische Untersuchung war bei allen vier Patienten - mit Passage der Ileozökalklappe - komplikationslos durchgeführt worden. In den Tabelle 19 - 22 werden diese Patienten vorgestellt. 


\section{Fall 1: 60-jährige Patientin}

Tabelle 19: Fallvorstellung 1: follikuläres B-Zell-Lymphom des Dünndarms

\begin{tabular}{|c|c|}
\hline $\begin{array}{l}\text { Initiale Anamnese } \\
(01 / 2007)\end{array}$ & rezidivierende Oberbauchbeschwerden ohne B-Symptomatik \\
\hline Nebendiagnosen & NIDDM, HLP, Adipositas per magna (BMI 49) \\
\hline Medikamente & Pantozol $40 \mathrm{mg}$ \\
\hline $\begin{array}{l}\text { Vorunter- } \\
\text { suchungen und } \\
\text { Staging (extern) }\end{array}$ & $\begin{array}{l}\text { ÖGD (02/2007): Duodenalpolyp-Ektomie, } \\
\text { keine Mukosektomie; Histologie: Lymphom } \\
\text { - Abdomen-Sonografie, Koloskopie, CT-Thorax und Abdomen, } \\
\text { Knochenmarkbiopsie (02/ 2007): ohne wegweisenden Befund }\end{array}$ \\
\hline Staging (intern) & $\begin{array}{l}\text { - Sonografie }(02 / 2007) \text { : } \\
\text { Hepatomegalie, Steatosis hepatis, Cholezystolithiasis } \\
\text { ÖGD (02/2007): Polyp im Duodenum pars descendens: } \\
\text { makroskopisch V.a. NHL; Histologie: Lymphangiektasie, } \\
\text { keine Malignität } \\
\text { - Dünndarmdoppelkontrastdarstellung nach Sellink (02/2007): } \\
\text { unauffällig }\end{array}$ \\
\hline $\begin{array}{l}\text { Indikation für } \\
\text { Kapselendoskopie }\end{array}$ & Polyp- und Tumorsuche \\
\hline $\begin{array}{l}\text { VKE-Befund } \\
(02 / 2007)\end{array}$ & $\begin{array}{l}\text { - } \text { Erosionen im } 1 / 3+3 / 3 \\
\text { - } 2 \text { zystische Lymphangiektasien im } 2 / 3 \\
\text { - } \quad \text { verplumptes bis atrophiertes Zottenrelief im } 2 / 3 \\
\text { - } \quad \text { leichte Schleimhautrötung im } 2 / 3 \\
\text { - } \quad \text { lymphozytäre Infiltrate und Lymphfollikel } \\
\text { - } \quad \text { keine Polypen, keine Stenose } \\
\text { - } \quad \text { V.a. Lymphombefall des oberen und mittleren Dünndarms }\end{array}$ \\
\hline $\begin{array}{l}\text { Nachunter- } \\
\text { suchungen }\end{array}$ & $\begin{array}{l}\text { - Leberbiopsie (02/2007) wegen unklaren Transaminasenanstiegs: } \\
\text { geringgradige portale Hepatitis, aszendierende Cholangitis und } \\
\text { Leberverfettung, toxischer Leberparenchymschaden, } \\
\text { kein Lymphom } \\
\text { - Enteroskopie (02/2007) im tiefen Dünndarm: } \\
\text { solitäre gerötete Schleimhautareale mit Ödem } \\
\rightarrow \text { Histologie: niedrig-malignes NHL }\end{array}$ \\
\hline Diagnose & follikuläres B-Zell-Lymphom des Dünndarms, Malignitätsgrad I \\
\hline Procedere & $\begin{array}{l}\text { - } \quad \text { abwartendes Verhalten } \\
\text { - Kontroll-ÖGDs (ggf. Kontroll-Enteroskopien) alle } 3 \text { Monate }\end{array}$ \\
\hline
\end{tabular}


Abb. 37: Fall 1

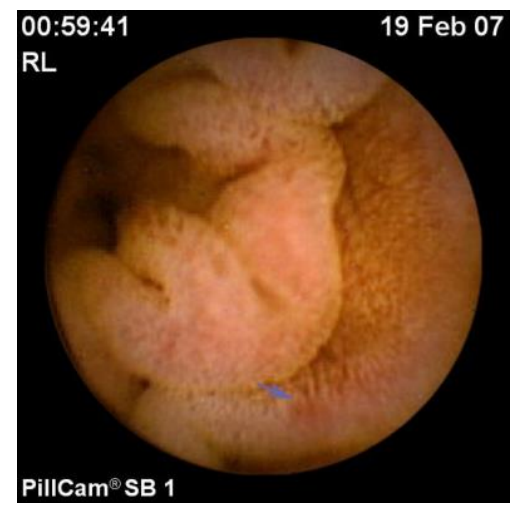

verplumptes Zottenrelief mit weißlichen Stippchen

\section{Fall 2: 44-jähriger Patient}

Tabelle 20: Fallvorstellung 2: VKE nach Jejunumsegmentresektion bei GIST

\begin{tabular}{|c|c|}
\hline $\begin{array}{l}\text { Initiale Anamnese } \\
(05 / 2010)\end{array}$ & $\begin{array}{l}\text { - } \text { stärkste kolikartige Schmerzen im linken Unterbauch } \\
\text { - } \\
\text { - } \\
\text { Eigdomen: palpatorisch bretthart } \rightarrow \text { akutes Abdomen } \\
\text { Diarrhoe } \\
\text { - } \quad \text { keine Gewichtsabnahme (BMI 23), keine B-Symptomatik } \\
\text { - } \quad \text { V.a. gedeckt perforierte Sigmadivertikulitis } \\
\text { - } \\
\text { Not-Operation }(05 / 2010): \text { Peritonitis bei Ruptur eines GIST- } \\
\text { Tumors im Jejunum } \rightarrow \text { Dünndarmsegmentresektion }\end{array}$ \\
\hline Diagnose & $\begin{array}{l}\text { GIST des Jejunums (Größe: } 7 \text { x } 7 \text { x } 4 \text { cm, Mitoserate: } 2 \text { pro } 50 \text { HPF) } \\
\text { mittlerer Malignitätsgrad, ED 05/2010 }\end{array}$ \\
\hline $\begin{array}{l}\text { Medikamente } \\
\text { postoperatives }\end{array}$ & 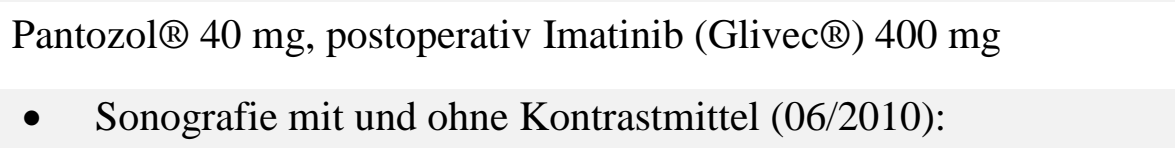 \\
\hline Staging & $\begin{array}{l}\text { kleine Läsion rechter Leberlappen (V.a. Hämangiom), } \\
\text { kein Hinweis auf abdominelle Raumforderung } \\
\text { - ÖGD (06/2010): chronische Gastritis und Bulbitis, } \\
\text { kleiner submuköser Tumor in der Pars descendens duodeni; } \\
\rightarrow \text { Histologie: gering- bis mäßiggradige chronische Duodenitis, } \\
\text { keine Malignität }\end{array}$ \\
\hline
\end{tabular}


- Koloskopie (06/2010): Zökumpolypektomie R0

$\rightarrow$ Histologie: sessiles serratiertes Adenom in toto, low-grade IEN

- Enteroskopie - oraler Zugangsweg (06/2010):

keine komplette Dünndarmpassage möglich, Bestätigung des kleinen Tumors in der Pars descendens duodeni

- Ganzkörper PET-CT mit 18-FDG (06/2010): Pars descendens duodeni: Tumor-suspekter Befund, entzündliche LK hiliär, solitäre kleine Lungenherde am ehesten Granulome

Indikation zur Polyp- und Tumorsuche im Dünndarm

Kapselendoskopie

VKE-Befund

$(06 / 2010)$

Nachunter-

suchungen

Diagnose

(1 Monat nach

VKE)
- $\quad$ vergröbertes Zottenrelief mit kleinen Erosionen im 1/3

- keine Polypen, keine Stenose, keine Blutungszeichen

- $\quad$ kein Hinweis für Raumforderung

- Ganzkörper PET-CT mit 18-FDG (12/2006): unter Imatinib kein Hinweis auf Resttumor oder Tumorrezidiv

- ÖGD (12/2006): insgesamt unauffällige Schleimhaut Zustand nach GIST-Resektion des Jejunums ohne Rezidiv im GIT

Abb. 38: Fall 2

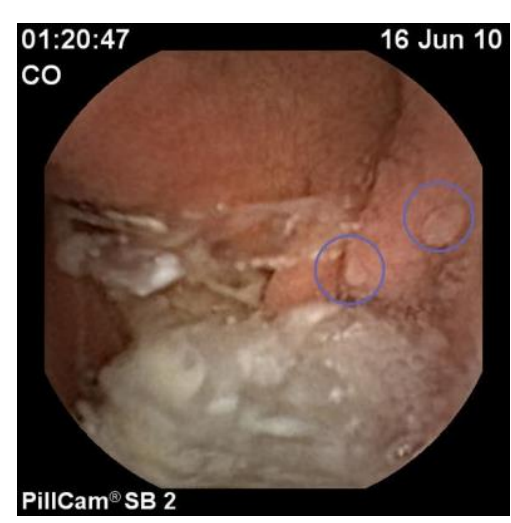

Dünndarmbefund bei dem Patienten mit Zustand nach GIST, spezifische Veränderungen mit vergröbertem Zottenrelief und zwei kleinen Erosionen (blaue Umkreisungen) 


\section{Fall 3: 40-jähriger Patient}

Tabelle 21: Fallvorstellung 3: Metastasen bei malignem Melanom im Jejunum

\begin{tabular}{|c|c|}
\hline $\begin{array}{l}\text { Initiale Anamnese } \\
(08 / 2006)\end{array}$ & $\begin{array}{ll}\text { - } & \text { Gewichtsabnahme von } 4 \mathrm{~kg} \text { in } 4 \text { Wochen, BMI } 23 \\
\text { - } & \text { Müdigkeit, Hautblässe } \\
\text { - } & \text { Hämoccult-Test positiv }\end{array}$ \\
\hline Vorgeschichte & $\begin{array}{l}\text { - noduläres malignes Melanom an der Haut rechts abdominell, } \\
\text { Clark-Level IV, ED 11/2000 mit Primär- und Nachexzision } \\
\text { - Z.n. Lymphadenektomie links inguinal 01/2002, 10/2005 } \\
\text { - Z.n. Exzision einer subkutanen Metastase am Rücken 01/2006 } \\
\text { und einer kutanen Metastase rechts axillär 02/2006 } \\
\text { - Z.n. Exzision einer intramuskulär gelegenen Metastase } \\
\text { am mittleren Unterbauch 06/2006 } \\
\text { - neue Lymphknotenmetastasen retroperitoneal/ paraaortal } \\
\text { (CT-Abdomen/ Becken) 05/2006 }\end{array}$ \\
\hline Medikamente & $\begin{array}{l}\text { - Pantozol } 20 \mathrm{mg} \\
\text { - Z.n. mehrfacher DTIC-Monotherapie 05-11/2002, 06 + 07/2006 } \\
\text { Immuntherapie mit Interferon 11/2002 bis 12/2004 und } \\
01-05 / 2006\end{array}$ \\
\hline
\end{tabular}

Staging

- Sonografie $(01+06+08 / 2006)$ : paraaortale Raumforderung, lateral der linken Niere: inhomogene Struktur

DD: LK-Metastase/ vom Darm ausgehende Raumforderung

- CT-Abdomen (06/2006): zwei kleine Leberzysten, Splenomegalie, am ehesten konfluierende RF im linken Mittelbauch - Jejunum und Colon descendens, Komprimierung der Jejunalvene, V.a. Tumoreinbruch ins Darmlumen, retroperitoneale und iliakale LK-Metastasen

- CT-Abdomen mit Kontrastmittel (08/2006): metastasenverdächtiger Tumor im Dünndarm und Perforation des Darmlumens

- zerebrales MRT (01/2006):

kein Hinweis auf zerebrale Metastasen 
- ÖGD (08/2006): suspektes, livide verfärbtes submukös gelegenes Areal im tiefen Duodenum

- Koloskopie (08/2006): kein Hinweis auf Tumor/ Blutungsquelle

Indikation zur

Kapselendoskopie

VKE-Befund

$(08 / 2006)$

Procedere unklare gastrointestinale Blutung, V.a. Metastase im Jejunum bzw. Tumoreinbruch ins Darmlumen

$\rightarrow$ hämorrhagisch ulzerierter Tumor im oberen Jejunum

- multiple kleine nicht-ulzerierte tumorverdächtige Strukturen im gesamten Dünndarm

- $\quad$ keine bedeutsame Stenose

- Teilresektion Jejunum und Colon descendens (08/2006):

$10 \mathrm{~cm}$ langes Tumorrezidiv mit Infiltration des Jejunums und linken Kolons

- 2 Zyklen DTIC-Chemotherapie (09 - 10/2006)

- polytope Lebermetastasierung (10/2006), massiver Progress suprapubischer, inguinaler und iliakaler Lymphknotenmetastasen (10/2006)

- Radiatio der Lymphknotenkonglomerate bis 50 Gy $(11-12 / 2006)$

- Exitus letalis fünf Monate (01/2007) nach VKE an Multiorganversagen Tumorrezidiv bei malignem Melanom im Dünndarm 
Abb. 39: Fall 3

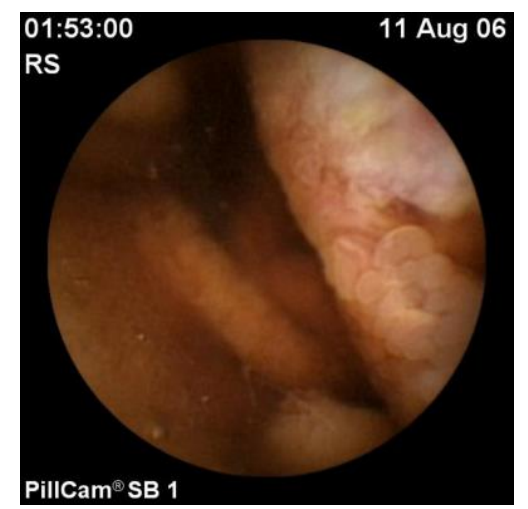

V.a. ulzeröse Metastase am duodenojejunalen Übergang

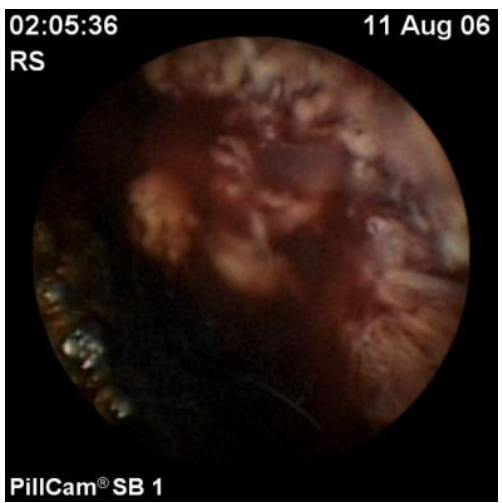

hämorrhagisch ulzerierter

Tumor im oberen Jejunum

\section{Fall 4: 65-jähriger Patient}

Tabelle 22: Fallvorstellung 4: Sarkommetastase im Jejunum

\begin{tabular}{|c|c|}
\hline $\begin{array}{l}\text { Initiale Anamnese } \\
(07 / 2007)\end{array}$ & $\begin{array}{ll}\text { - } & \text { Teerstuhl, Hämoccult-Test positiv } \\
\text { - } & \text { Anämie } \\
\text { - } & \text { Belastungsdyspnoe bei Zigarettenabusus } 35 \text { py } \\
\text { - } & \text { kein Gewichtsverlust, BMI } 19\end{array}$ \\
\hline Vorgeschichte & $\begin{array}{l}\text { - } \\
\text { destdiagnose 05/2007: sarkomatoides Weichteilsarkom } \\
\text { - } \quad \text { Lymphknotenmetastase links axillär } \\
\text { - } \quad \text { COPD bei Lungenemphysem } \\
\text { - } \quad \text { Gefäß-KHK mit Z.n. 4-fach ACVB }(05 / 2007) \\
\text { - } \quad \text { Z.n. Alkohol-Abusus } \\
\text { - } \quad \text { Z.n. Appendektomie }\end{array}$ \\
\hline Medikamente & ASS $®$, Niedermolekulares Heparin, Pantozol ${ }^{\circledR} 40$ mg \\
\hline Voruntersuchungen & $\begin{array}{l}\text { - Sonografie (06/2007): Leberzyste und Leberhämangiom, } \\
\text { keine weiteren Raumforderungen } \\
\text { - } \quad \text { ÖGD + Koloskopie (06/2007): unauffällig, } \\
\text { keine Blutungsstigmata, keine Blutungsquellen }\end{array}$ \\
\hline
\end{tabular}




\begin{tabular}{|c|c|}
\hline $\begin{array}{l}\text { Indikation zur } \\
\text { Kapselendoskopie }\end{array}$ & Hämoglobin-relevante unklare gastrointestinale Blutung \\
\hline $\begin{array}{l}\text { VKE-Befund } \\
(07 / 2007)\end{array}$ & $\begin{array}{ll}\text { - } & \text { kleine Angiodysplasien, Erosionen, Ulzerationen im } 1 / 3 \\
\text { - } & \text { frische Blutspuren im } 1 / 3 \\
\text { Ulzeration mit aktiver Blutung im 1/3; } \\
\text { - } \quad \text { sumor nicht sicher auszuschließen } \\
\text { ohne Stenose und Blutungszeichen im } 2 / 3 \\
\rightarrow \quad \text { keine Polypen, keine Stenose } \\
\quad \text { V.a. ulzerierenden Tumor als Blutungsquelle } \\
\text { im oberen Dünndarmdrittel }\end{array}$ \\
\hline Nachuntersuchungen & $\begin{array}{l}\text { Doppelballonenteroskopie bis } 220 \mathrm{~cm} \text { ab Treitzband } \\
\text { (07/2007): histologische Sicherung der ulzerierenden, } \\
\text { tumorösen Veränderung mit Blutungsstigmata } \\
\text { in der Umgebung } \\
\text { PET-CT mit 18-FDG (07/2007): tumoröses Geschehen } \\
\text { im distalen Colon descendens } \\
\text { - ÖGD (08/2007): unauffällige Schleimhaut }\end{array}$ \\
\hline Procedere & $\begin{array}{l}\text { - Segmentresektion (07/2007) bei Tumorblutung einer } \\
\text { Jejunummetastase } \\
\text { - } \quad \text { Exitus letalis zwei Monate nach VKE (09/2007) } \\
\text { wegen respiratorischer Insuffizienz }\end{array}$ \\
\hline & Sarkommetastase im Jejunum \\
\hline
\end{tabular}

Abb. 40: Fall 4 - I
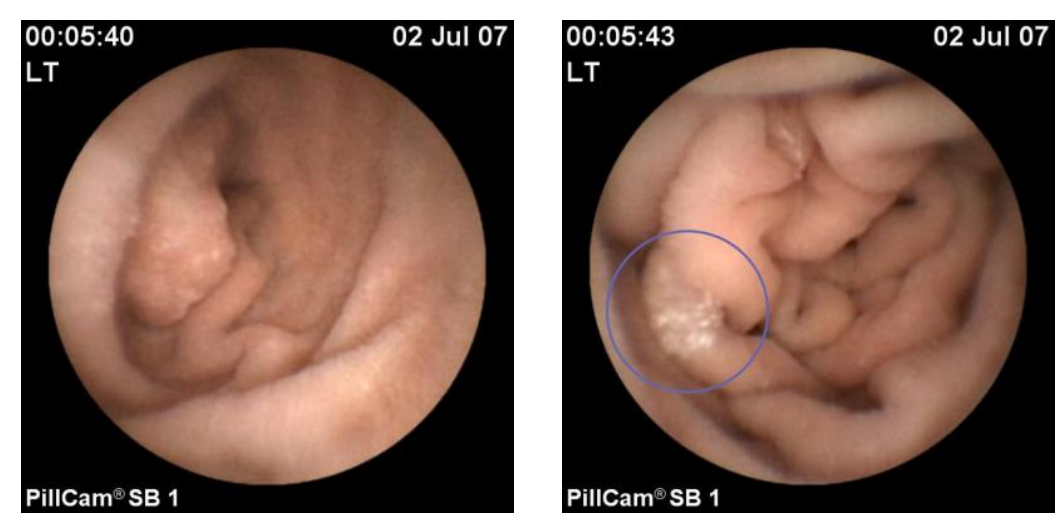

lymphozytäre Infiltration der Schleimhaut im oberen Anteil/ DD: Lymphangiektasien 
Abb. 41: Fall 4 - II

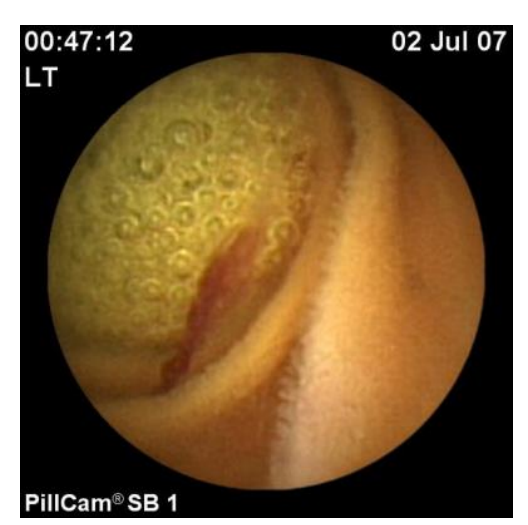

frische kleine Blutspur im Dünndarm

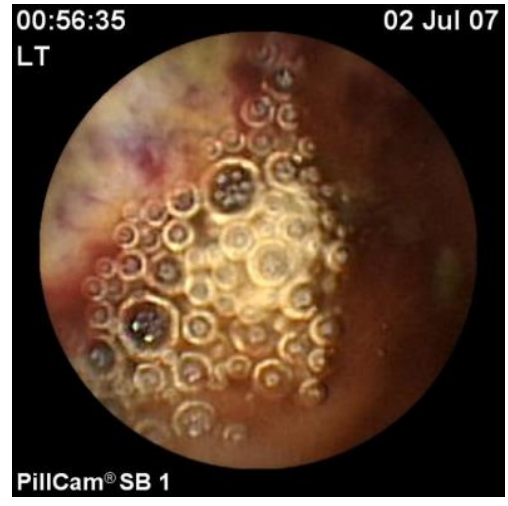

Schleimhaut-Veränderung mit Blutungszeichen

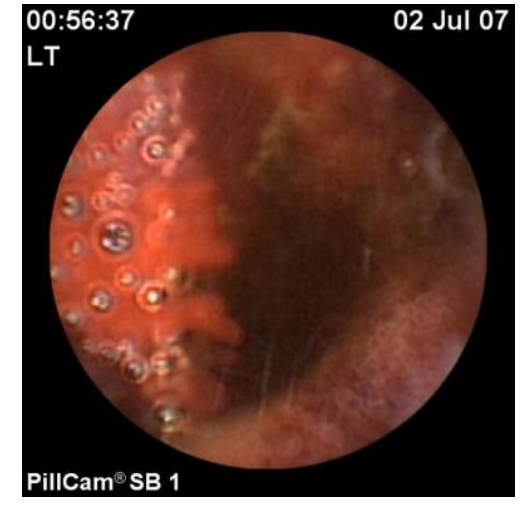

ulzerierende Veränderung mit aktiver Blutung 


\subsection{Nachuntersuchungen}

Bei 35\% des Patientenkollektivs (71/203) schlossen sich nach der Videokapselendoskopie weitere Untersuchungen an. Einige Patienten $(n=13)$ erhielten davon mehrere Untersuchungen. Aus den Patientenakten war ersichtlich, dass bei fünf Patienten (7\%) eine Abdomen-Sonografie durchgeführt wurde. Eine Wiederholungs-Gastroskopie fand bei zehn Patienten $(11,9 \%)$ statt. Eine erneute Koloskopie erfolgte bei 17 Patienten (24\%). Sechs Patienten zeigten koloskopisch keine auffälligen Befunde. Bei drei weiteren Patienten konnte mittels einer bioptischen Sicherung ein Adenokarzinom der Dickdarmschleimhaut gefunden werden. Eine Sigmadivertikulose wurde bei vier Patienten diagnostiziert. Ein Patient zeigte Blutungszeichen, jedoch konnte bei ihm keine Blutungsquelle detektiert werden. Der letzte der 17 koloskopisch nachuntersuchten Patienten wies eine hochgradige entzündliche Stenose im terminalen Ileum auf.

Eine Single-Ballon-Enteroskopie erfolgte bei vier Patienten (5,6\%). Zwei davon hatten eine Blutung. Bei den anderen zwei Patienten, bei denen ein Verdacht auf eine bösartige Erkrankung geäußert wurde, konnte bioptisch das histologische Ergebnis eines niedrig-malignen NonHodgkin-Lymphoms bzw. eines ulzerierenden, undifferenzierten Malignoms im Dünndarm im Sinne einer Jejunummetastase diagnostiziert werden (vgl. Falldarstellungen: Tabelle 22).

Die weiteren Nachuntersuchungen sind in der nachfolgenden Abb. 42 veranschaulicht.

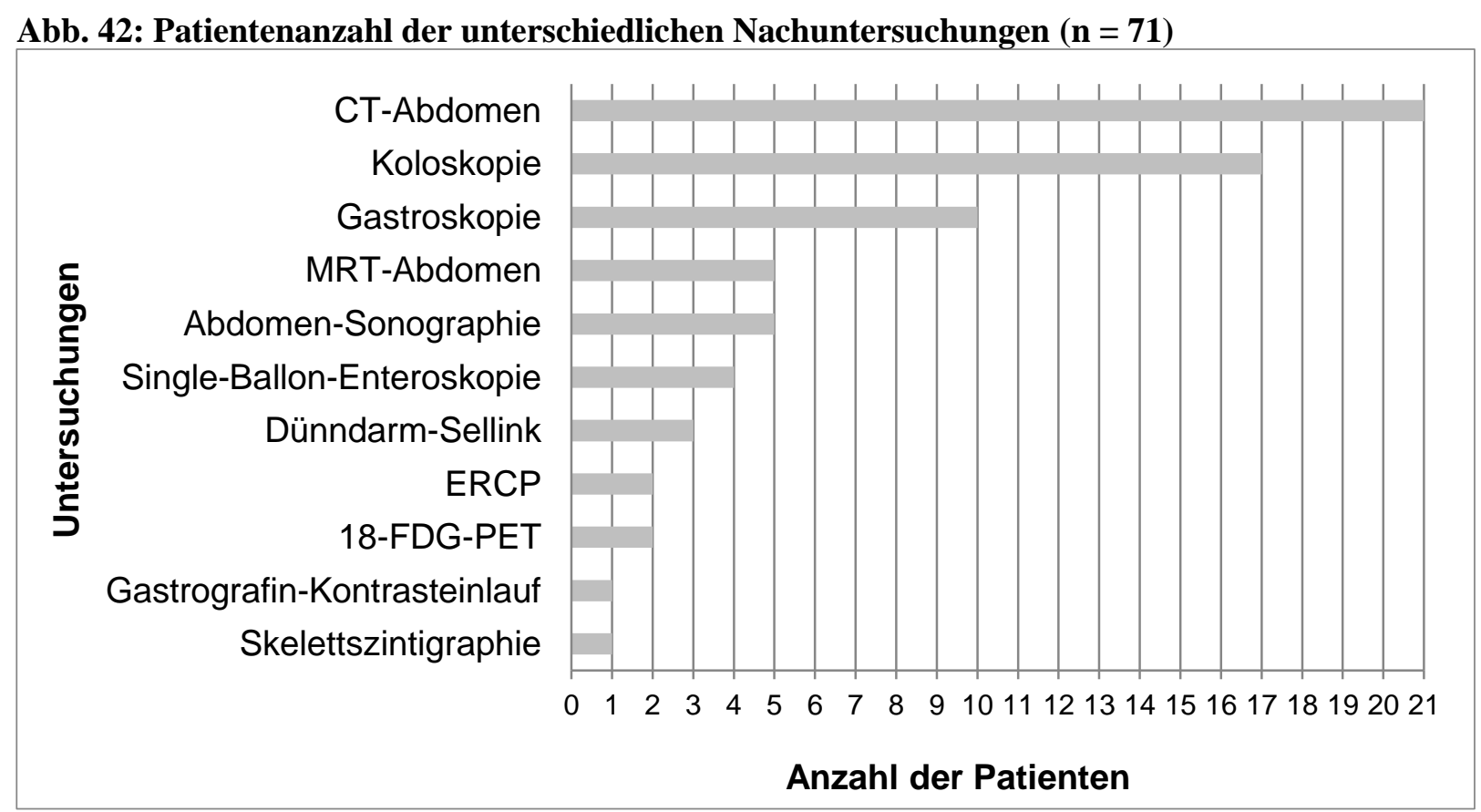




\subsection{Therapien nach VKE}

Bei 203 durchgeführten Videokapselendoskopien ergaben sich für rund drei Viertel der untersuchten Patienten [73,4\% (149/203)] therapeutische Konsequenzen. Etliche Patienten erhielten mehrere unterschiedliche Therapien: von der medikamentösen (über die endoskopische) bis hin zur chirurgischen Therapie.

Diese therapeutischen Maßnahmen wurden zu 64\% (59/92) bei Patienten mit der Indikation unklare gastrointestinale Blutung durchgeführt, zu 69,6\% (16/23) bei Patienten mit unklarer Anämie. Ein Patient mit gastrointestinalen Blutungen erhielt neben den o.g. Therapiearten eine radiologisch-interventionelle Therapie (TIPS-Anlage) bei portaler Hypertension.

\subsubsection{Medikamentöse Therapie}

Eine medikamentöse Therapie resultierte bei 66\% der Göttinger Patientenklientel (133/203) aus den Videokapselendoskopie-Befunden. 35\% (47/133) erhielten davon eine Mehrfachkombination der Medikamente. Aus der Tabelle 23 sind die von den Ärzten verordneten Medikamente ersichtlich.

Tabelle 23: Medikamente ( $n=$ Anzahl der Patienten, welche die Arzneimittel erhielten)

\begin{tabular}{l|c}
\hline Analgetika & 4 \\
Antibiotika & 10 \\
Eisenpräparate & 60 \\
Erythrozytenkonzentrate & 10 \\
Fresh Frozen Plasma & 9 \\
HP-Eradikationstherapie & 3 \\
Immunsuppressiva (Azathioprin) & 9 \\
Mesalazin & 14 \\
Muskelrelaxans & 39 \\
Prokinetikum (MCP) & 30 \\
Protonenpumpenhemmer & 8 \\
Reduktion/ Pause der gerinnungsbeeinflussenden Medikamente & 1 \\
Steroide & 2 \\
Thalidomid & \\
\hline
\end{tabular}


Eine medikamentöse Therapie wurde bei 58,7\% der Patienten (54/92) mit unklaren gastrointestinalen Blutungen und bei 65,2\% der Patienten (15/23) mit unklaren Anämien angewandt.

18 der 27 Dünndarmblutungspatienten (66,7\%) erhielten therapeutisch Erythrozytenkonzentrate $(\mathrm{EK})(\mathrm{p}=0,0005)$, drei unter ihnen zusätzlich Fresh Frozen Plasma-Präparate (FFP) $(\mathrm{p}=0,04)$. Von den EK-Einheiten wurden zwei bis maximal zehn $(\overline{\mathrm{x}}=4 \pm 2,4)$, von den FFP-Einheiten ein bis maximal zehn $(\overline{\mathrm{x}}=4 \pm 4,9)$ verabreicht.

Da 60 von 203 Patienten (29,6\%) eine bedeutsame Anämie mit einem durchschnittlichen Hämoglobinwert von 7,6 g/dl (3,7-10 g/dl) hatten, wurden ihnen 1 bis 30 Erythrozytenkonzentrate während des stationären Aufenthaltes verabreicht. 16 der 60 Patienten erhielten vier EKEinheiten, 22 Patienten zwei EK-Einheiten. Insgesamt wurden den 60 Patienten 225 Erythrozytenkonzentrate appliziert ( $\overline{\mathrm{x}}=4,25 \mathrm{EK}$ 's pro Anämie-Patient).

Zehn (16,7\%) dieser 60 Patienten bekamen insgesamt 37 Einheiten FFP verabreicht. Sieben Patienten (70\%) erhielten zwei FFP-Konzentrate, die restlichen drei Patienten (30\%) jeweils ein, zwei oder zwölf Konzentrate.

\subsubsection{Endoskopie mit Interventionen}

Eine interventionelle Endoskopie erhielten 4\% der Patienten (8/203), sechs von ihnen hatten die Indikation unklare gastrointestinale Blutung.

Zwei Gastroskopien waren mit Blutstillung bei Ösophagusvarizen und Angiodysplasien von therapeutischer Bedeutsamkeit.

Die Single-Ballon-Endoskopie erfolgte bei vier Patienten (4,8\%). Zwei davon hatten eine Blutung, die erfolgreich thermisch mit Argon-Plasma koaguliert werden konnte.

Bei einem weiteren Patienten erfolgte eine Kolonpolypektomie. Trotz einer hochgradigen Stenosierung im terminalen Ileum konnte bei einem anderen Patienten die Videokapsel ileokoloskopisch geborgen werden. 


\subsubsection{Operative Therapie}

Eine Operation fand bei 4,4\% der Patienten (9/203) aufgrund ihres pathologischen Videokapselbefundes statt. Fünf dieser neun Patienten hatten die Indikation unklare gastrointestinale Blutung, drei Patienten unklare Bauchschmerzen und ein weiterer Patient einen Morbus Crohn-Verdacht.

Anlass für die Operation waren in der vorgeschalteten Koloskopie festgestellte pathologische Befunde oder die Kapselbergung.

Bei hochgradig entzündlicher Stenose im terminalen Ileum misslang bei einem koloskopisch nachuntersuchten Patienten der endoskopische Bergungsversuch. Daraufhin erfolgte eine therapeutische Dünndarmsegmentresektion des Jejunums und Ileums mit Entfernung der festgesteckten Videokapsel. Weitere Dünndarmsegmentresektionen des Jejunums wurden bei drei Patienten durchgeführt: Bei zwei Patienten aufgrund von Metastasen, bei dem dritten Patienten wegen Adhäsionen.

Eine partielle Dickdarmresektion erfolgte bei vier anderen Patienten, zwei Mal fand bei diagnostizierten Rektumkarzinomen eine tiefe anteriore Rektumresektion statt. Bei zwei anderen Patienten wurde eine Hemikolektomie rechts durchgeführt. Hier war die Operation indiziert wegen einer Kolon-Perforation nach Polypektomie und das andere Mal zur Resektion eines Adenokarzinoms im rechten Kolon.

Bei dem letzten der zehn Patienten wurde eine Gastrektomie wegen rezidivierender Magenschleimhautblutungen bei portaler hypertensiver Gastropathie als Ultima Ratio durchgeführt. 


\section{Diskussion}

Die nicht-invasive Videokapselendoskopie wird als Goldstandard in der Diagnostik von Dünndarmerkrankungen angesehen. Folgende Fragen zur VKE werden in diesem Kapitel diskutiert.

1. Wird durch eine optimale Vorbereitung die Kapselauswertung vereinfacht?

2. Beeinflussen die Vorerkrankungen der Patienten die Passagezeit der Videokapsel?

3. Können die diagnostischen Fragen, die zur VKE-Untersuchung geführt haben, beantwortet werden?

4. Ist die Dünndarmkapselendoskopie trotz der bekannten Kontraindikationen eine risikoarme Methode den Dünndarm zu visualisieren?

5. War der Aufwand der VKE-Untersuchung trotz der erforderlichen Nachuntersuchungen und den sich ggf. anschließenden Therapien gerechtfertigt und kostenverträglich?

6. Mit welchen Methoden kann man ähnliche Ergebnisse wie mit der VKE erzielen?

7. Wird sich die VKE in Zukunft noch stärker etablieren?

\subsection{Vorbereitung der VKE}

Laut Kapselhersteller Given Imaging® besteht die Vorbereitung für die VideokapselUntersuchung aus einer Diät mit klaren Flüssigkeiten sowie einer acht- bis zwölf-stündigen Nahrungskarenz. Jedoch konnte 2009 in der Metaanalyse von Rokkas (bestehend aus sechs prospektiven und sechs retrospektiven Studien mit insgesamt 1.162 Patienten) gezeigt werden, dass Abführmittel von Vorteil sind, da sie erstens eine bessere Sicht auf die Dünndarmmukosa ermöglichen [281/404 (69,5\%) vs. 135/249 (54,2\%) Patienten ohne Darmlavage, $p=0,005]$ und zweitens zu einer größeren diagnostischen Ausbeute führen [122/263 (46,3\%) vs. 80/213 $(37,5 \%)$ Patienten ohne Vorbereitung, $p=0,002$ ] (Rokkas et al. 2009).

Trotzdem werden die Patienten in vielen gastroenterologischen Arztpraxen ohne eine vorherige Einnahme von Abführmedikamenten kapselendoskopiert und müssen stattdessen lediglich eine Ernährungspause von 12 bis 24 Stunden einhalten. Momentan existieren keine Standardmaßnahmen für die Vorbereitung der Dünndarmkapselendoskopie (Ladas et al. 2010). Manche Untersucher empfehlen die Einnahme von zwei Liter eines PEG-Abführmittels, andere 
bevorzugen eine komplette Koloskopie-Vorbereitung (Koornstra 2009; Triantafyllou et al. 2008).

Vorbereitungsstandard für das Universitätsklinikum Göttingen ist die Säuberung des Gastrointestinaltraktes mit stuhlabführenden Maßnahmen: ein bis zwei Liter eines PEG-Präparates - abhängig von der Anordnung des untersuchenden Arztes - in Kombination mit einer Nahrungskarenz von 20 Stunden, siehe Kapitel 2.4.3.

Darunter verfügten 145 (71,3\%) von 203 Patienten über eine gute Beurteilbarkeit des Dünndarms. Bei ihnen konnte eine optimale Videokapselauswertung stattfinden. Eine eingeschränkte Auswertung lag bei 51 Patienten $(25,2 \%)$ wegen Verstuhlung oder Blutauflagerungen vor. Bei zwei Patienten (1\%) war die VKE bei zu starker Verschmutzung erfolglos.

Studien konnten belegen, dass mit zwei und vier Liter PEG-Lösung die gleiche Sauberkeit des Dünndarms erreicht werden kann. Auch bestand kein Unterschied in der Dünndarmpassagezeit während der VKE, wenn die Patienten mit zwei oder vier Liter PEG-Lösung vorbereitet wurden (Kantianis et al. 2009; Park et al. 2011). Dais Arbeitsgruppe fand 2005 heraus, dass Abführmaßnahmen im Rahmen der VKE die Dünndarmpassagezeiten verringern (213 vs. 253 Minuten ohne Darmlavage, $\mathrm{p}<0,01$ ) (Dai et al. 2005). Im Gegensatz dazu konnte in Rokkas' Studie kein signifikanter Unterschied zwischen der Darmlavage und einer Diät aus klaren Flüssigkeiten bezüglich der Magen- und Dünndarmpassagezeit sowie dem vollständigen Durchlaufen der Kapsel durch den Dünndarm während der Aufnahmezeit [469/563 (83,3\%) vs. 288/355 (81,1\%), $\mathrm{p}=0,665]$ detektiert werden (Rokkas et al. 2009).

Nicht nur die Stuhlverschmutzung behindert die Auswertung der Videokapsel, auch Luftblasen können die Sicht auf die Dünndarmschleimhaut verringern. Um dieses Problem zu minimieren, werden Entschäumer wie Simeticon empfohlen (Albert et al. 2004; Ge et al. 2006). Die Kombination, bestehend aus oraler Darmlavage und Simeticon, erbringt bei Wei und Fang die besten Studienergebnisse $(p<0,001)$ bei der Detektion von pathologischen Befunden bzw. der Beurteilung der Dünndarmmukosa (Fang et al. 2009; Wei et al. 2008). Nach Fangs Studie beeinflusst Simeticon die Magenpassagezeit kaum. Die Dünndarmpassagezeit wird durch Simeticon verlängert (von 227,28 auf 281,84 Minuten; $p=0,003$ ) (Fang et al. 2009).

Der routinemäßige Einsatz von Prokinetika zur Vermeidung einer Gastroparese und zum Erreichen des Zökums während der VKE wird in Studien hinterfragt. Vier Arbeiten zeigten bei Verwendung des Medikamentes Erythromycin eine Verkürzung der Magenpassage, aber keinen Effekt auf die Dünndarmpassagezeit bzw. das Erreichen der Ileozökalklappe (Caddy et al. 2006; Fireman et al. 2005; Leung et al. 2005; Niv et al. 2008). 
In Selbys prospektiver, nicht randomisierter Studie konnte nachgewiesen werden, dass durch die Verabreichung von $10 \mathrm{mg}$ Metoclopramid (MCP) eine verringerte Magentransitzeit $(30,8 \pm 7,5$ vs. 47,9 $\pm 9,0$ Minuten ohne MCP; $\mathrm{p}=0,025)$ und ein besseres Erreichen des Zökums gewährleistet wird [65/67 (97\%) vs. 63/83 (76\%); p < 0,001] (Selby 2005). Die randomisierte Arbeit von Almeida zeigte hingegen keinen signifikanten Unterschied in der Rate der kompletten Dünndarmuntersuchungen mit oder ohne MCP auf [38/47 (80,9\%) vs. $37 / 48(77,1 \%)$; $\mathrm{p}=0,422]$ (Almeida et al. 2010). Die Arbeitsgruppe um Apostolopoulos berichtet über einen originellen Weg, die Passagezeit zu beeinflussen. Die Hälfte der randomisierten Probanden (47/93 Patienten) kauten alle 2 Stunden für 30 Minuten einen Kaugummi. Bei dieser Gruppe konnte das Zökum häufiger visualisiert werden [39/47 (83\%) vs. 33/46 (71,7\%); p=0,19]. Die Magen(40,8 vs. 56,1 Minuten) wie auch die Dünndarmtransitzeit (229,1 vs. 266,2 Minuten) waren signifikant geringer $(\mathrm{p}<0,05)$ als in der Kontrollgruppe (Apostolopoulos et al. 2008).

Die VKE ist eine kosten- und zeitintensive Prozedur (Dauer der Auswertung ca. 1-2 Stunden). Deshalb sollte sie unter optimalen Voraussetzungen stattfinden, damit die beste diagnostische Ausbeute erreicht werden kann.

Es sollte obligat darauf geachtet werden, dass die Patienten vor der Einnahme der Kapsel einen wässrigen bzw. klar-flüssigen Stuhlgang haben. Auch wenn die Abführmaßnahmen von den meisten Patienten als unangenehm und störend empfunden werden, steht der Nutzen, die Dünndarmschleimhaut gut sichtbar zu machen, im Vordergrund. 


\subsection{Videokapselpassagezeit}

\subsubsection{Videokapselpassagezeit bezüglich Alter und BMI}

Die 203 Patienten des Göttinger Patientenkollektivs, bestehend aus 118 Männern (58\%) und 85 Frauen (42\%), weisen einen Altersdurchschnitt von $58( \pm 19,5)$ Jahren und einen BMI-Median von 24,8 auf.

Die Studie von Velayos Jimenez zeigte ähnliche Ergebnisse mit einem Durchschnittsalter der Patienten $(\mathrm{n}=89)$ von 59,2 Jahren und einem BMI-Median von 25,5 (Velayos Jimenez et al. 2005).

Die Magenpassagezeiten des nachweisbaren Göttinger-Kollektivs $(n=173)$ lag zwischen 1 und 462 Minuten $(\overline{\mathrm{x}}=21$ Minuten). Auch die Patienten der Velayos Jimenez-Studie hatten annähernd gleiche Magenpassagezeiten mit einem Median von 22,6 Minuten und lagen zwischen 0,7 und 171 Minuten (Velayos Jimenez et al. 2005). Die Dünndarmtransitzeit der ermittelbaren Göttinger Patienten $(\mathrm{n}=170)$ betrug zwischen 18 und 522 Minuten $(\overline{\mathrm{x}}=245$ Minuten) lang. Hier lag bei der Velayos Jimenez Studie der Median der Dünndarmpassagezeit $(\overline{\mathrm{x}}=283,3$ Minuten, 91-416 Minuten) höher als beim Göttinger Kollektiv (Velayos Jimenez et al. 2005).

Bei den Göttinger Patienten über 60 Jahre oder bei BMI-Werten über 25 ließ sich keine Signifikanz bezüglich längerer Magen- oder Dünndarmpassagezeiten erkennen (jeweils $p=0,9$ ). Genauso wurden in der Studie von Velayos Jimenez keine signifikanten Unterschiede zwischen den Magen- und Dünndarmpassagezeiten und dem Alter, Geschlecht, BMI oder dem Bauchumfang gefunden (Velayos Jimenez et al. 2005).

\subsubsection{Videokapselpassagezeit bei Diabetes mellitus-Patienten}

Bei 29 Patienten (14,3\%) des Göttinger Kollektivs war eine Diabetes mellitus-Erkrankung bekannt. Sie wiesen eine geringere Magenpassagezeit (1-139 Minuten, $\bar{x}=13$ Minuten) als das gesamte Göttinger Kollektiv (1-462 Minuten, $\bar{x}=21$ Minuten) auf. Die 144 Patienten ohne Diabetes mellitus hatten hingegen eine längere Magentransitzeit (1-462 Minuten, $\overline{\mathrm{x}}=22$ Minuten). In der Triantafyllou-Studie $(\mathrm{n}=87)$ war die Magenpassage der Diabetes mellitus-Patienten $(\mathrm{n}=29)$ im Gegensatz zu dem gleichgroßen Göttinger Kollektiv deutlich länger (1-478 Minuten, $\overline{\mathrm{x}}=87$ versus 13 Minuten). Dasselbe gilt bei den Triantafyllou-Patienten 
ohne Diabetes mellitus $(\mathrm{n}=58) \quad(\overline{\mathrm{x}}=24$ versus 22 Minuten; $4-108$ Minuten; $\mathrm{p}<0,001)$ (Triantafyllou et al. 2007).

Bei den Göttinger Diabetes mellitus-Patienten $(n=27)$ lag die Dünndarmpassagezeit (15470 Minuten, $\overline{\mathrm{x}}=216$ Minuten) ebenso wie die Magenpassagezeit unter der Zeit der Patienten ohne Diabetes mellitus ( $\mathrm{n}=143)$ (48-525 Minuten, $\overline{\mathrm{x}}=255$ Minuten).

$\mathrm{Da}$ in Studien bekannt ist, dass Diabetes mellitus-Patienten eine längere Magenpassagezeit aufweisen, erreichen die Kapseln in der bisherigen Literatur seltener die Ileozökalklappe als bei Vergleichspatienten ohne Diabetes mellitus. Triantafyllous Studie zeigte, dass 69\% (20/29) der Videokapseln der Diabetes mellitus-Patienten diese Klappe erreichten. In der Kontrollgruppe ohne Diabetes mellitus konnten bei Triantafyllou 89,6\% (52/58) der Videokapseln das Zökum visualisieren (Triantafyllou et al. 2007). In der Göttinger Studie gelangten 93\% der Videokapseln (27/29) bei Diabetes mellitus-Patienten durch diese Klappe, die Kapseln der Patienten ohne Diabetes mellitus passierten zu 99\% (143/144) die Klappe. Auch bei den Triantafyllou-Patienten war die Dünndarmpassagezeit (wie bei den Göttinger Diabetes-Patienten) signifikant kürzer $(\overline{\mathrm{x}}=261,2 \pm 55,5$ versus 216 Minuten $)$ als die 55\% der Patienten ohne Diabetes $(32 / 58)(\overline{\mathrm{x}}=302$ $\pm 62,7$ versus 255 Minuten; $p=0,03$ ) (Triantafyllou et al. 2007).

Jedoch ist bei der Auswertung der verschiedenen Magen- und Dünndarmpassagezeiten der unterschiedliche Größenunterschied der Patientengruppe mit und ohne Diabetes mellitus zu beachten. Für eine genaue Aussage, welche Patientengruppen eine kürzere oder längere Magenoder Dünndarmtransitzeit haben, sollte die Studienpopulation eine gleiche Größe aufweisen.

\subsubsection{Videokapselpassagezeit und Komorbidität}

Die Vermutung, dass Patienten mit Diabetes mellitus oder mit Erkrankungen des Magen-DarmTraktes eine längere Magenpassagezeit aufweisen würden, konnte bei der Göttinger Population nicht nachgewiesen werden. Neben den Göttinger Diabetes mellitus-Patienten wiesen auch die Patienten mit Erkrankungen der Bauchorgane oder vorherigen Operationen im Magen-DarmTrakt keine längere Magentransitzeit auf. 


\subsection{Indikationsbezogene Ergebnisse}

In den Videokapselendoskopien der Göttinger Patientenpopulation wurden bei 85,2\% (173/203) pathologische Ergebnisse im Dünndarm entdeckt. Bei 11\% der Patienten (22/203) wurden keine pathologischen Veränderungen gefunden. Bei $4 \%$ der Patienten (8/203) fehlten diagnostische Angaben. Im Vergleich dazu wies die Rondonotti-Studie zu 50,6\% (1477/2921) pathologische Auffälligkeiten sowie zu 36\% (1051/2921) fehlende Pathologien auf, in 13,4\% (393/2921) waren die Läsionen nicht sicher einzuordnen (Rondonotti et al. 2010). Die Nachweisrate von Liaos Studie zeigte zu 59,4\% pathologische Befunde in der Kapselendoskopie (Liao et al. 2010). In der Rondonotti-Studie zählten die Angiodysplasien mit 52,2\% (771/1477) zu den häufigsten pathologischen Befunden (Rondonotti et al. 2010). Auch in dem Göttinger Patientengut waren die kapillären Läsionen (Angiodysplasien, Petechien) zu 43,9\% (76/173) und somit bei etwa der Hälfte der Patienten mit pathologischen Befunden zu finden.

Ein Vergleich der nachfolgend zitierten Studien findet sich in tabellarischer Form auf S. $105 \mathrm{ff}$.

\subsubsection{Gastrointestinale Blutung}

Die häufigste Indikation für die Videokapselendoskopien war bei der Göttinger Patientenpopulation mit 45,3\% (92/203 Patienten) die unklare gastrointestinale Blutung. In der Multicenterstudie von Rondonotti war diese Indikation mit 43,3\% (1268/2921) ähnlich hoch vertreten (Rondonotti et al. 2010). Nach Liaos Literaturübersicht lag deren höchste Indikationsrate mit 66\% (14623/22840) höher als die Göttinger oder Rondonotti-Studie (Liao et al. 2010). Auch in Toys Studie standen die gastrointestinalen Blutungen als Indikationen an erster Stelle [overte Blutungen: 38\% (55/145), okkulte Blutungen: 22\% (32/145)] (Toy et al. 2008)

Die diagnostische Ausbeute des Göttinger Kollektivs lag bei der genannten Indikation bei 80,4\% (74/92). In der Studie von Pennazio konnten nur zu 47\% (47/100) Auffälligkeiten im Dünndarm durch die VKE detektiert werden (Pennazio et al. 2004). Diese Studiengruppe zeigte, dass die diagnostische Ausbeute bei der Indikation unklare gastrointestinale Blutung von der Art der Blutung abhängt. Die höchste diagnostische Ausbeute mit 92\% konnte bei den Patienten erzielt werden, die eine anhaltende sichtbare Blutung hatten. Die niedrigste Ausbeute mit $13 \%$ lag bei Patienten mit einer stattgehabten overten Blutung vor (Pennazio et al. 2004). Die RondonottiStudie wies eine höhere diagnostische Ausbeute mit 62,5\% (793/1268) als die Pennazio-Studie 
auf (Rondonotti et al. 2010). Eine fast gleich hohe diagnostische Ausbeute von 60,5\% (8847/14623) konnte die Liao-Studie erbringen (Liao et al. 2010).

Bei den Göttinger Patienten mit gastrointestinaler Blutung standen die Schleimhautläsionen mit 47,8\% (44/92) an erster Stelle. Gegensätzlich dazu waren in Liaos Studie Angiodysplasien zu 50\% (1375/2751) für die gastrointestinalen Blutungen verantwortlich (Liao et al. 2010). In der Pennazio-Studie konnten nur zu 29\% (29/100) Angiodysplasien und zu 6\% (6/100) M. CrohnLäsionen als Blutungsquelle detektiert werden (Pennazio et al. 2004).

Der positive prädiktive Wert für die Auswertung der unklaren gastrointestinalen Blutung mit intestinalen Läsionen liegt bei Delvaux bei $94 \%$ und bei Pennazio bei $97 \%$. Der negative prädiktive Wert für normale Schleimhautverhältnisse wird von Delvaux und Pennazio mit $83 \%$ bzw. 100\% angegeben (Delvaux et al. 2004; Pennazio et al. 2004).

Die Kapselendoskopie vermag die Blutungsläsionen zu identifizieren und kann zu weiteren therapeutischen Interventionen oder chirurgischen Eingriffen verhelfen. Die Nachblutungsrate der Kapselendoskopien ohne pathologische Auffälligkeiten liegt bei 5,6\% (1/18), die derjenigen mit pathologischen Veränderungen bei 48,4\% (15/31) (Lai et al. 2006).

Neben Pennazio vertreten wir die Meinung, dass bei einer unklaren gastrointestinalen Blutung die Videokapselendoskopie zügig nach einer Gastro- und Koloskopie ohne pathologischen Befund zur weiteren Diagnostik durchgeführt werden sollte. Bei einem detektierten pathologischen Befund kann danach eine entsprechende Therapie begonnen werden. Zusätzlich kann durch den schnellen VKE-Einsatz eine Kostenersparnis durch verkürzte Diagnostikzeiten und ggf. auch verkürzte Krankenhausliegedauer erreicht werden (Pennazio et al. 2004).

\subsubsection{Unklare abdominelle Schmerzen}

Die Indikation unklare abdominelle Schmerzen war der zweithäufigste Grund für die VKE bei den Göttinger Patienten. 24,1\% der Patienten (49/203) teilten diese Indikation. Auch in Toys Studie stand die Indikation mit 20,7\% (30/145) an zweiter Stelle (Toy et al. 2008). Eine geringere Indikationsrate lag bei Liaos Studie vor, dort waren 10,6\% (2358/22840) der Patienten mit klinischen Symptomen vertreten (Liao et al. 2010). Die Rondonotti-Studie wies die geringste Rate auf [5,3\% (155/2921)] (Rondonotti et al. 2010). Die höchste Rate [55\% (35/64)] fand sich in der Studie von Fry. Diese Studie untersuchte nur Patienten mit unklaren abdominellen Schmerzen, unklarer Diarrhoe oder mit beiden Symptomen (Fry et al. 2006). 
Die diagnostische Ausbeute der Göttinger Patienten mit der Indikation unklare abdominelle Schmerzen lag bei 40,8\% (20/49). Sowohl in der Studie von Rondonotti [14,9\% (23/155)] als auch bei Fry [6\% (2/35)] war die diagnostische Ausbeute deutlich geringer (Fry et al. 2006; Rondonotti et al. 2010). Für diese Indikation waren keine weiteren Angaben zu den detaillierten Ursachen und Therapien in der Studie von Rondonotti oder Fry zu finden (Fry et al. 2006; Rondonotti et al. 2010). Die Göttinger Patienten wiesen bei dieser Indikation folgende pathologische Befunde auf: Erosionen bei 12\% (6/49), fibrinbelegte Ulzerationen bei 8\% (4/49), Angiektasien bei 8\% (4/49), Angiodysplasien bei 4\% (2/49), aphthöse Läsionen bei 4\% (2/49) und eine Divertikelblutung zu 2\% (1/49).

Die DGVS empfiehlt wegen der geringen Ausbeute in der VKE, bei den Patienten mit unklaren Bauchschmerzen zuerst die Basisuntersuchungen (ÖGD, Koloskopie, Schnittbildgebung des Abdomens inklusive Sonografie und radiologischer Diagnostik) vorzunehmen und bei unklaren oder negativen Ergebnisse dann die VKE (May et al. 2010). Unsere Ergebnisse unterstützen dieses Vorgehen: Erstens um das Risiko der Kapselretention zu minimieren, zweitens um für den Patienten überflüssige Untersuchungen zu vermeiden.

\subsubsection{Unklare Anämie}

Die Videokapselendoskopie erfolgte bei den Göttinger Patienten zu 11,3\% (23/203) aufgrund der Indikation unklarer Anämie. Sie stellt damit den dritthäufigsten Grund für die VKE in unserem Patientengut dar. Eine höhere Indikationsrate [23,9\% (698/2921)] besaß die Studie von Rondonotti. Hier war die unklare Anämie der zweithäufigste Grund für die Kapselendoskopie (Rondonotti et al. 2010). Eine geringere Indikationsrate wies hingegen die Cheon-Studie mit 6,4\% (82/1291) auf (Cheon et al. 2007). In der Studie von Toy wurde die VKE nur bei 2,8\% (4/145) der Patienten mit dieser Indikation durchgeführt (Toy et al. 2008).

Eine diagnostische Ausbeute der unklaren Anämie konnte in der UMG bei 78\% (18/23) der Patienten detektiert werden. Die Studie von Apostolopoulos, an der nur Patienten mit unklarer Anämie teilnahmen, zeigte eine fast gleich hohe diagnostische Ausbeute von 56,9\% (29/51). In der Rondonotti-Studie lag die diagnostische Ausbeute bei 40,9\% (285/698) (Rondonotti et al. 2010).

Ursächlich für die Anämie waren bei den Göttinger Patienten in erster Linie Erosionen, Ulzerationen sowie Angiodysplasien. Bei Apostolopoulos konnten zu 23,5\% (12/51) Angiodysplasien als Grund für die unklare Anämie gefunden werden, zu 17,6\% (9/51) multiple 
oder solitäre Ulzerationen, zu 7,8\% (4/51) Erosionen, zu 3,9\% (2/51) Polypen sowie wiederum zu 3,9\% (2/51) Tumoren im Jejunum (ein Adenokarzinom sowie eine Metastase eines malignen Melanoms) (Apostolopoulos et al. 2006).

Weitere detaillierte Angaben $\mathrm{zu}$ den Ursachen und Therapien bzw. den diagnostischen Ausbeuten konnten in den Studien von Rondonotti, Cheon und Toy nicht gefunden werden.

Ein Algorithmus für die Indikation unklare Anämie wird wie folgt von Apostolopoulos empfohlen: Nach einer unauffälligen Gastro- und Koloskopie mit unauffälligen Duodenalbiopsien sollte eine VKE erfolgen (Apostolopoulos et al. 2006). Diesem Algorithmus schließen wir uns an.

\subsubsection{Verdacht auf Morbus Crohn}

Die Indikation Verdacht auf M. Crohn führte bei 4\% der Göttinger Patienten (8/203) zu der VKE. Bei Cheons Studie war diese Indikation nur bei 2,9\% (37/1291) der Patienten vertreten (Cheon et al. 2007). In der Studie von Rondonotti erfolgte die VKE bei 7,8\% (228/2921) dieser Patienten (Rondonotti et al. 2010).

Die Göttinger Patienten mit Verdacht auf M. Crohn wiesen eine diagnostische Ausbeute von 62,5\% (5/8) auf. Zu den pathologischen Befunden zählten: Schleimhautläsionen [75\% (6/8)], Ulzerationen $[75 \%$ (6/8)], zystische Lymphangiektasien [50\% (4/8)] sowie Lumenveränderungen [37,5\% (3/8)]. Bei 47,3\% (108/228) der Patienten in Rondonottis Studie konnten auffällige Befunde in der VKE detektiert werden (Rondonotti et al. 2010). Genaue Angaben über die Art der pathologischen Befunde fehlten in dieser Studie.

Nach der DGVS soll eine VKE bei Patienten mit einem M.Crohn-Verdacht, bei denen die klinischen und laborchemischen Befunde passen, aber die endoskopischen Untersuchungen nicht ausreichend waren, durchgeführt werden (May et al. 2010). Diese Meinung vertreten auch wir, um unnötige Wartezeiten auf diagnostische Maßnahmen zu umgehen und somit ggf. eine schnellere Therapie zu beginnen. Voraussetzung sollte der vorangegangene Ausschluss einer Passagestörung sein. 


\subsubsection{Morbus Crohn}

Eine hohe Bedeutsamkeit hat die VKE bei der Diagnose M. Crohn. Im Göttinger Kollektiv wurde sie bei 2,5\% (5/203) der Patienten mit M. Crohn durchgeführt. Ähnlich hohe Indikationsraten wurden in der Studie von Rondonotti und Cheon gesehen: Bei Rondonotti war diese Indikation zu 3,7\% (108/2921) vorhanden (Rondonotti et al. 2010), in Cheons Studie zu 1,8\% (23/1291) (Cheon et al. 2007). Höhere Indikationsraten als in den drei genannten Studien kamen in der Studie von Liao und Toy vor. Die Indikationsrate lag in Liaos Studie bei 10,4\% (2295/22840) (Liao et al. 2010) und in Toys Studie bei 12,4\% (18/145) (Toy et al. 2008).

Pathologische Befunde konnten in der UMG bei $80 \%$ (4/5) gefunden werden. Die M. CrohnPatienten der Rondonotti-Studie wiesen eine geringere diagnostische Ausbeute von 54,8\% (59/108) auf (Rondonotti et al. 2010). In Frys Studie ( $=64)$, die ausschließlich Patienten mit unklarer Diarrhoe, unklaren abdominellen Schmerzen oder mit beiden Symptomen einschloss, wurde die Diagnose M. Crohn bei 4,7\% (3/64) der Patienten gestellt (Fry et al. 2006).

Die VKE zeigte bei den fünf M. Crohn-Patienten aus Göttingen zu 60\% (3/5) Schleimhautläsionen einschließlich Ulzerationen auf. In der Rondonotti-Studie konnten auch hier keine Angaben zu den pathologischen Befunden der M. Crohn-Patienten gefunden werden. Lediglich konnten entzündliche Veränderungen wie Ulzerationen und Erosionen im Dünndarm bei der Gesamtanzahl der Patienten zu 20,9\% (309/1477) durch die VKE entdeckt werden (Rondonotti et al. 2010). Die Studie von Liao zeigte bei 55,3\% (1269/2295) dieser Patienten pathologische Auffälligkeiten (Liao et al. 2010). Nähere Angaben fehlten auch hier zu der Art der Befunde.

Die DGVS weist auf den hohen Stellenwert der VKE im Nachweis einer M. Crohn-Erkrankung hin, da sie eine höhere Sensitivität als die radiologischen Verfahren (Enteroklysma, MRT oder CT) besitzt. Es ist zu beachten, dass die NSAR-Enteropathie ähnliche Befunde wie die durch M. Crohn verursachten mukosalen Läsionen zeigen kann (May et al. 2010).

Eine Aussage zum Nutzen der Videokapselendoskopie bei der Crohn-Indikation ist bei lediglich 2,5\% der Betroffenen unserer Patientenklientel nicht möglich. 


\subsubsection{Unklare Diarrhoe}

Das Symptom unklare Diarrhoe führte bei 6,4\% der Patienten (13/203) an der UMG zur Videokapselendoskopie. Eine geringere Indikationsrate wies hingegen zu 4,5\% (58/1291) die Cheon-Studie auf (Cheon et al. 2007). Bei der Rondonotti-Studie war eine ähnliche Rate von 4,8\% (140/2921) vorhanden (Rondonotti et al. 2010). In Frys Studie lag die Indikationsrate bei $22 \%$ (14/94). Die gemeinsame Indikation unklare abdominelle Schmerzen und unklare Diarrhoe war bei weiteren $23 \%$ (15/64) vorhanden (Fry et al. 2006).

In der UMG erbrachte die VKE eine diagnostische Ausbeute von 46\% (6/13) bei den Patienten mit unklarer Diarrhoe. Im Gegensatz dazu war eine niedrigere diagnostische Ausbeute mit 27,4\% (38/140) in der Rondonotti-Studie vorhanden (Rondonotti et al. 2010). Die pathologischen Befunde der Indikation chronische Diarrhoe (Diarrhoe > 5x/d: > 6 Wochen) wiesen auch in Frys Studie einen geringen Wert auf. $\mathrm{Zu} \mathrm{14 \%} \mathrm{(2/14)} \mathrm{der} \mathrm{Fälle} \mathrm{konnte} \mathrm{eine} \mathrm{Ursache}$ gefunden werden (Fry et al. 2006).

$\mathrm{Zu}$ den Befunden zählten bei den Göttinger Patienten: aphthöse Läsionen [15\% (2/13)], Lymphangiektasien [15\% (2/13)], fibrinbelegte Ulzeration [8\% (1/13)] sowie eine Erosion und Zottenatrophie [8\% (1/13)]. In der Cheon-Studie konnte bei einem Durchfall-Patienten eine M. Crohn-Erkrankung durch die VKE detektiert werden (Cheon et al. 2007).

Eine Empfehlung für die Kapselendoskopie bei der Indikation unklarer Diarrhoe ist bei $46 \%$ positiven Befunden zurückzustellen.

\subsubsection{Polyp- und Tumorsuche}

Die Polyp- und Tumorsuche erfolgte bei 5,4\% der Göttinger Patienten (11/203) durch die VKE. In Rondonottis Studie waren 3,4\% der Patienten (101/2921) mit einem Verdacht auf einen Dünndarmtumor vertreten. Zusätzlich wiesen 3,1\% der Patienten (90/2921) ein PolyposisSyndrom auf, welches kapselendoskopisch beurteilt werden sollte (Rondonotti et al. 2010). In der Studie von Liao wurde die VKE bei 3,5\% der Patienten (786/22840) mit tumorösen Veränderungen durchgeführt (Liao et al. 2010).

Eine Pathologie konnte bei 90,9\% der Göttinger Patienten (10/11) in der VKE gefunden werden. Die diagnostische Ausbeute der Patienten mit Verdacht auf einen Dünndarmtumor war in Rondonottis Studie mit 41\% (41/101) geringer. Bei den Polyposis-Patienten lag sie bei 74\% (67/90) (Rondonotti et al. 2010). Eine präzise Indikationsstellung hilft zur Ausbeute bei genauer Betrachtung. 
Die Patienten der Rondonotti-Studie wiesen zu 16\% (235/1477) Polypen oder tumoröse Veränderungen auf (Rondonotti et al. 2010). In Liaos Studie wurden bei 55,9\% der Patienten mit Polyp- und Tumorsuche (439/786) tumoröse Veränderungen nachgewiesen (Liao et al. 2010). In Frys Studie $(\mathrm{n}=64)$ konnte unter den Indikationen unklare abdominelle Schmerzen mit und ohne die Indikation unklare Diarrhoe ein submukosaler Tumor (Karzinoid) durch die VKE detektiert werden (Fry et al. 2006).

Ein Dünndarmtumor konnte bei $1,5 \%$ der Göttinger Patienten $(3 / 203 ; \overline{\mathrm{x}}=55$ Jahre) bestätigt werden. Bei einem weiteren Patienten unseres Kollektivs konnte durch die VKE eine zusätzliche Tumormanifestation des Primärtumors GIST ausgeschlossen werden. Eine der drei Tumorpatienten litt bei lymphozytären Infiltraten und Lymphfollikeln nachweislich an einem niedrigmalignen NHL. Ein anderer Patient war an einem malignen Melanom erkrankt, er zeigte einen hämorrhagisch ulzerierenden Tumor als Metastase im Jejunum. Der dritte Patient wies auch einen ulzerierenden Tumor im Jejunum bei der Grunderkrankung Lungensarkom auf. Die Patientin hatte die Indikation Polyp- und Tumorsuche, die zwei Männer die Indikation unklare gastrointestinale Blutung. In den Voruntersuchungen (ÖGD, Ballon-Enteroskopie und CTAbdomen) konnten die tumorverdächtigen Dünndarmläsionen zwar gesehen werden, jedoch konnten sie durch die VKE besser morphologisch eingeordnet werden.

In Baileys Literaturübersicht über 416 Kapselendoskopien wurden 27 Tumoren bei 26 Patienten $(6,3 \% ; \bar{x}=61 \pm 13,7$ Jahren) detektiert. Indikationen für die VKE waren bei 21 Patienten die unklare gastrointestinale Blutung, bei drei Patienten ein Tumorverdacht, bei einem Patient unklare abdominelle Schmerzen und bei einem weiteren Patienten die Diarrhoe. Radiologische Voruntersuchungen wiesen in acht von 23 Fällen (35\%) eine mögliche Läsion nach. Neun Tumoren waren benigne und 17 maligne. $\mathrm{Zu}$ den malignen Tumoren zählten die Adenokarzinome ( $\mathrm{n}=5$ ), Karzinoide (6), Melanommetastasen (2), GIST (2), Kolonkarzinommetastase (1), NHL (1). Die benignen Tumoren waren mit Hamartomen (4), zystischen Lymphangiomen (1), Amyloidtumoren (1) sowie Lipome (1) vertreten (Bailey et al. 2006).

Aus Rondonottis Multicenterstudie von 2008 (5129 VKE) gingen 124 Dünndarmtumoren (2,4\%) hervor. Unter diesen Patienten bestanden die Indikationen: obskure gastrointestinale Blutung (108 Patienten), unklare abdominelle Schmerzen (9), Polyp- und Tumorsuche (6) sowie Diarrhoe mit Malabsorption (1). Im Vorfeld fand bei 55 Patienten $(44,4 \%)$ eine unauffällige bidirektionale Endoskopie statt. Zusätzliche Dünndarmuntersuchungen zeigten bei 24 von den anderen 69 Patienten $(55,6 \%)$ eine Pathologie. 
Durch die VKE konnten 80,6\% der Tumoren detektiert werden. 112 Dünndarmtumoren waren maligne und zwölf Tumoren benigne. Der vorherrschende primäre Dünndarmtumor war der gastrointestinale Stromatumor (GIST) (32\%), gefolgt vom Adenokarzinom (20\%) und Karzinoid (15\%). $\mathrm{Zu}$ den sekundären Tumoren zählten mit 66\% vorwiegend die Melanommetastasen (Rondonotti et al. 2008).

Da die Dünndarmtumoren sich erst spät manifestieren und dann zu 50\% bereits metastasiert sind (Minardi et al. 1998), besitzt die VKE ein hohes Potential, die Diagnose Dünndarmtumor früher zu stellen, wenn die Patienten erste Symptome zeigen. Der Nachteil der VKE ist, dass kein histologischer Befund mit Bestimmung einer Tumorentität möglich ist (Bailey et al. 2006).

In unserem Patientengut betrug die Ausbeute bei der Indikation Tumorsuche im Dünndarm $18,2 \%(2 / 11)$.

\subsubsection{Einzelfallindikationen}

Da die Indikationen rezidivierendes unklares Erbrechen und das Eiweißverlustsyndrom bei jeweils einem Patienten des gesamten Göttinger Kollektivs vorhanden waren, werden diese hier gemeinsam betrachtet. Bei beiden Patienten konnte die VKE zu einer Diagnosestellung führen. Einmal wurde eine Duodenitis bei weißlich verdickten Dünndarmvilli gezeigt. Das andere Mal wurde eine primär intestinale Lymphangiektasie bei ödematösen Dünndarmvilli beschrieben. In der Literatur konnten keine genauen Angaben zu diesen Indikationen gefunden werden. 


\subsection{Komplikationen der Dünndarmkapselendoskopie}

\subsubsection{Schluckstörung}

$\mathrm{Zu}$ den leichten Komplikationen zählen laut den Studien von Almeida und Carey ( $n=18$ Patienten) Schwierigkeiten beim Schlucken der Videokapsel, die einen endoskopischen Transfer nach sich ziehen. Als Kontraindikation sehen sie die Unmöglichkeit des Schluckens der Kapsel, eine Magenretention bei einer vorherigen VKE-Untersuchung sowie pathologische Veränderungen des oberen Gastrointestinaltraktes an (Almeida et al. 2008; Carey et al. 2004).

Eine seltene Komplikation stellt die Kapselaspiration dar, von der bisher nur Einzelfallberichte existieren (Koulaouzidis et al. 2009; Nathan und Biernat 2007). Laut Koulaouzidis scheint das Alter ein Risikofaktor für diese Art von Aspiration zu sein. Deshalb sollen die Kapseln seiner Meinung nach in Reanimationsbereitschaft eingenommen werden. Zudem sollte die Möglichkeit einer Sauerstoffzufuhr bestehen. Wird ergänzend ein Real-Time Viewer verwendet, kann zeitgleich gesehen werden, wo sich die Videokapsel befindet.

Um die genannten Komplikationen oder Kontraindikationen des Schluckens zu minimieren, wird in der Literatur die Kapselapplikation bei Patienten mit Schluckbeschwerden oder bei Kindern per ÖGD in den Bulbus duodeni empfohlen (Güldütuna und Keuchel 2009, S. 22). 14 Patienten (7\%) des Göttinger Patientenkollektivs erhielten eine solche Anwendung. Bei fünf jungen Patienten (8-16 Jahre) mit den Indikationen: Polyposis-Syndrom, Verdacht auf Morbus Crohn oder auf intestinalen Eiweißverlust wurde diese Applikation durchgeführt. Die anderen neun Kapselapplikationen erfolgten bei erwachsenen Patienten bei Zustand nach Magenresektion sowie aufgrund von physischen oder psychischen Schluckstörungen.

\subsubsection{Dünndarmkapselretention}

Die größte Gefahr der Videokapselendoskopie besteht in der Dünndarmkapselretention.

Unter den 203 Patienten des Göttinger Patientenkollektivs litten 2\% (4/203) an dieser Komplikation. Drei Patienten waren an M. Crohn (1,5\%) und ein Patient an FAP $(0,5 \%)$ erkrankt. In Rondonottis Studie lag mit 2,1\% (61/2921) eine ähnliche Gesamtretentionsrate vor. Hier wurden 32 Videokapseln (1\%) 15 Tage nach der VKE auf natürlichem Weg ohne Therapie ausgeschieden. Die verbliebenen 29 Kapseln (0,99\%) wurden per Endoskopie oder Chirurgie geborgen. In zwei Fällen (0,07\%) erfolgte eine Not-Operation aufgrund einer akuten Obstruktion 
(Rondonotti et al. 2010). In Cheons Multicenterstudie $(n=1291$ Patienten $)$ belief sich die Retentionsrate auf 2,5\% (32/1291) und lag somit nur unwesentlich höher (Cheon et al. 2007). Bei elf Patienten $(0,85 \%)$ erfolgte die Kapselbergung vor Symptombeginn endoskopisch oder chirurgisch. Die verbliebenen 21 Patienten $(1,6 \%)$ wurden zunächst medikamentös behandelt. 10 dieser 21 Patienten erhielten bei intestinaler Obstruktion oder dem Versagen der medikamentösen Therapie eine chirurgische Kapselbergung. Bei den anderen elf Patienten gelang die medikamentöse Therapie und somit der spontane Kapselabgang (Cheon et al. 2007). In der Analyse von Li ereignete sich die Komplikation Kapselretention lediglich bei 1,4\% der Patienten (14/1000). Die Retention resultierte bei elf Patienten aus einer NSAR-Enteropathie (diaphragm disease), bei drei Patienten aus einem obstruierenden Tumor (Duodenum bzw. Ileum). Alle diese Patienten waren asymptomatisch. Bei 13 dieser Patienten erfolgte die Kapselentfernung durch eine elektive Dünndarmteilresektion (Li et al. 2008). In der Literaturübersicht von Liao mit einer VKE-Anzahl von 22840 lag die durchschnittliche Kapselretention ebenfalls bei 1,4\% (von 104 prospektiven und 46 retrospektiven Studien). 58,7\% der Videokapseln wurden davon durch die chirurgische Variante geborgen. 15,8\% der Kapseln wurden entweder spontan oder medikamentös unterstützt ausgeschieden. Weitere 12,5\% konnten endoskopisch gesichert werden (Liao et al. 2010).

In vielen Fällen lässt bereits die klinische Indikation der Kapselendoskopie auf eine höhere Retentionsrate schließen. Die Studie von Pennazio beobachtete bei gesunden Probanden keine Retention (Pennazio 2006). Demgegenüber liegt laut dem ICCE-Bericht von 2005 die Rate bei Patienten mit unklarer gastrointestinaler Blutung bei 1,5\% (15/1089) (Cave et al. 2005). In Liaos Multicenterstudie litten ähnlich viele Patienten (1,2\% aus 47 Studien) mit der gleichen Indikation an einer Retention (Liao et al. 2010). Marmos Metaanalyse erbrachte bei den Patienten $(n=526)$ mit der Indikation unklare gastrointestinale Blutung eine geringere Retentionsrate von 0,7\% (2/29 Patienten mit unerwünschten Ereignissen). Die Kapselbergung erfolgte bei dem ersten betroffenen Patienten auf dem endoskopischen, bei dem anderen auf dem chirurgischen Weg (Marmo et al. 2005). Die in der Literatur am häufigsten zitierte Studie von Barkin weist eine fast gleichwertige Retentionsrate von $0,75 \%$ (7/900 Patienten) bei der o.g. Indikation auf. Davon wiesen sechs Patienten in den Voruntersuchungen eine unauffällige Magen-Darm-Passage auf. Durch nachfolgende chirurgische Eingriffe konnte neben der Kapselbergung die pathologische Auffälligkeit (Tumoren und NSAR-Strikturen), welche die Retention verursachte, gefunden werden (Barkin und Frideman 2002). Von den Göttinger Patienten mit der Indikation unklarer gastrointestinaler Blutung erlitt keiner eine Kapselretention. 
Die Kapsel-Indikation M. Crohn weist im Vergleich zu der Indikation unklare gastrointestinale Blutung eine deutlich höhere Retentionsrate auf. Nach dem ICCE-Bericht lag die Retentionsrate bei bekanntem M. Crohn bei 5\% und bei Verdacht auf M. Crohn bei 1,4\% (Cave et al. 2005). Bei Patienten mit intestinaler Obstruktion konnte Pennazio eine Retentionsrate von $21 \%$ feststellen (Pennazio 2006). Andererseits lag in Cheifetz' Studie die Retentionsrate bei Patienten mit einem bekannten M. Crohn bei 13\%, bei V.a. M. Crohn bei nur 1,6\% (Cheifetz et al. 2006). Diese Studie selektierte die untersuchten 983 Patienten nach ihren Indikationen für die VKE und kam deshalb auf die hohen Retentionsraten. Von dieser Patientenanzahl wurden 102 Fälle wegen Verdacht auf M. Crohn $(n=64)$ oder einem bekannten M. Crohn $(n=38)$ einzeln betrachtet. Cheons Multicenterstudie kam bei den M. Crohn-Patienten auch auf eine Retentionsrate von 13\% (3/23), die Rate der Patienten mit V.a. M. Crohn lag hingegen bei 5,4\% (2/37) und somit höher als bei den Patienten der Cheifetz-Studie (Cheon et al. 2007). In der Metaanalyse von Marmo war die Retentionsrate der M. Crohn-Patienten mit 3\% wesentlich geringer als in den Studien von Cheifetz und Cheon (Cheifetz et al. 2006; Cheon et al. 2007; Marmo et al. 2005). Auch in Lis Studie wurden bei 3\% dieser Patientengruppe (7/237) Kapselretentionen dokumentiert (Li et al. 2008). Demgegenüber besaßen die M. Crohn-Patienten in der Studie von Liao eine Retentionsrate von 2,6\% (von 23 Studien) (Liao et al. 2010).

Die Göttinger Studie wies bei der Indikation M. Crohn eine niedrigere Retentionsrate von 1,5\% (3/203) als bei den oben genannten Studien auf. Die Kapselbergung erfolgte bei einem der drei betroffenen Patienten durch die Kombination Darmlavage und endoskopische Bergung (50 Tage nach VKE). Bei dem zweiten M. Crohn- Patienten ließ sich die Kapsel nach erfolgloser endoskopischer Intervention durch erneute Darmlavage zehn Tage nach der VKE ausscheiden. Bei dem dritten M. Crohn-Patienten misslang die bidirektionale Bergung 15 Tage nach der VKE. Aufgrund eines akuten Abdomens erfolgte hier eine notfallmäßige Laparotomie mit Kapselbergung.

Darüber hinaus kann bei den Patienten mit der Indikation Polyp- und Tumorsuche im Dünndarm die Komplikation Kapselretention auftreten. Rondonottis Multicenterstudie ( $\mathrm{n}=5129$ Videokapseln) zeigte bei 124 Patienten (2,4\%) einen Dünndarmtumor auf. 9,8\% dieser Patienten litten zusätzlich an einer Kapselretention (Rondonotti et al. 2008). In Cheons Multicenterstudie war die Retentionsrate aufgrund von gastrointestinaler Polyposis niedriger [3,6\% (1/28)] (Cheon et al. 2007). Bei Liaos Studie waren 2,1\% der Patienten mit tumorösen Veränderungen von Retentionen betroffen (Liao et al. 2010). In der Göttinger Studie trat eine Retention bei einem an FAP erkrankten Patienten auf. Die Bergung wurde neun Tage nach VKE endoskopisch durchgeführt. 
Die längste bisher in der Literatur beschriebene Retentionsdauer lag bislang bei 2,5 Jahren (Cave et al. 2005). 2013 wurde diese Retentionsdauer abgelöst durch einen asymptomatischen Patienten, bei dem die Kapsel über 4,5 Jahren im Magen-Darm-Trakt verblieb (Bhattarai et al. 2013). Bei dem Göttinger Kollektiv lag die maximale Zeit der Kapselretention hingegen bei 50 Tagen.

Zur Abschätzung des Retentionsrisikos wird empfohlen, vor dieser VKE-Untersuchung eine gründliche Patientenanamnese durchzuführen (Cave et al. 2005). In der Literatur werden folgende Ursachen für die Retention beschrieben: NSAR-Strikturen, M. Crohn, Dünndarmtumoren, Strahlenenteritis und chirurgische anatomische Strikturen. Ein potentielles Risiko für eine Retention besitzen Patienten mit unklaren abdominellen Schmerzen, lang andauernder NSAR-Einnahme sowie Patienten mit dem Verdacht auf oder der gesicherten Diagnose M. Crohn. Bei anamnestisch bekanntem Retentionsrisiko eignet sich eine radiologische Dünndarmbildgebung (z.B. CT-Sellink) oder die Patency-Kapsel, die sich nach entsprechender Vorbereitung im Dünndarm auflöst (Cave et al. 2005; Güldütuna und Keuchel 2009, S. 28).

Wenn eine Kapselretention eingetreten ist, verläuft die Retention meist asymptomatisch, selten birgt sie das Risiko der Dünndarmobstruktion (Cheifetz und Lewis 2006; Li et al. 2008).

Therapeutisch kann die Kapsel mit einer medikamentösen, endoskopischen oder chirurgischen Intervention entfernt werden. Der Chirurg kann nicht nur die Kapsel bergen, sondern darüber hinaus die pathologische Veränderung beheben, die zur Retention geführt und die VKE indiziert hat (Bhattarai et al. 2013).

Bei entsprechender Indikationsstellung ist die Untersuchung insgesamt sicher. Eine Aufklärung bezüglich dieses Risikos ist notwendig. 


\subsection{Voruntersuchungen der VKE}

Bei unserer Patientenpopulation $(n=203)$ liegt die durchschnittliche Rate an Voruntersuchungen bei 4,3 Untersuchungen pro Patient. Mit inbegriffen sind Laboruntersuchungen, Sonografien, Gastroskopien, Koloskopien, bildgebende Verfahren wie CT und MRT, Enteroklysmen, ERCP sowie Laparoskopien oder Laparatomien. In unseren Voruntersuchungen konnten teilweise pathologische Befunde detektiert werden. Zum Ausschluss oder Nachweis einer Dünndarmpathologie wurde daraufhin die VKE durchgeführt.

In der Studie von Cobrin fanden bei 50 Tumor-Patienten ähnlich viele Voruntersuchungen (4,2 pro Patient) statt. Darunter zählten Gastroskopien, Koloskopien, Push-Enteroskopien, Dünndarmpassagen mit Gastrografin und CT-Untersuchungen (Cobrin et al. 2006).

Die Metaanalyse von Lewis verglich Kapselendoskopien aus 24 Studien mit insgesamt 530 Patienten. Insgesamt erfolgten 6,77 Voruntersuchungen pro Patient ohne pathologische Befunde. 310 dieser 530 Patienten wiesen eine unklare gastrointestinale Blutung auf, bei ihnen wurden 7,4 diagnostische Untersuchungen vor der VKE durchgeführt. Folgende Voruntersuchungen fanden statt: Gastroskopien, Koloskopien, Dünndarmpassagen, Push-Enteroskopien, CT-Untersuchungen, Röntgen-Abdomen-Übersichten, Szintigrafien, Angiografien und intraoperative Enteroskopien (Lewis et al. 2005).

In der Studie von Pennazio $(\mathrm{n}=100)$ waren 40 Blutungspatienten im Vorfeld insgesamt 212-mal untersucht worden, sodass durchschnittlich 5,3 Untersuchungen pro Patient stattfanden. Diese erbrachten keinen pathologischen Befund. Zu den Untersuchungen gehörten: Gastroskopien, Koloskopien, Dünndarmpassagen, Enteroklysmen, CT-Angiografien, Arteriografien und Szintigrafien. Retrospektiv waren bei Pennazio die zusätzlich zu Gastroskopie und Koloskopie durchgeführten Voruntersuchungen nicht erforderlich, da sie keine über das Videokapsel-Ergebnis hinaus gehenden Befunde erbrachten (Pennazio et al. 2004). 


\subsection{Nachuntersuchungen der VKE und Therapie}

Bei 35\% des Göttinger Patientenkollektivs (71/203) erfolgten aufgrund der Befunde der Videokapselendoskopie weitere Untersuchungen: bildgebende Verfahren $(n=38)$ und Endoskopien $(n=33)$.

Therapeutische Konsequenzen ergaben sich bei 73\% unserer Patienten (148/203): Endoskopie mit Intervention $(n=6)$, Chirurgie $(n=9)$, die Mehrzahl dieser Patienten $(n=133)$ erhielt eine medikamentöse Therapie. Bei manchen Patienten wurden mehrere Therapiearten durchgeführt.

Die Ergebnisse der VKE führten bei 64\% der Göttinger Blutungsklientel (59/92) zu therapeutischen Maßnahmen: Endoskopie $(n=6)$, Chirurgie $(n=5)$, Medikamente $(n=54)$, radiologisch-interventionelle Therapie $(\mathrm{n}=1)$.

In der Studie von Pennazio $(n=100)$ wurde eine höhere Anzahl [88\% (80/91)] von Therapien durchgeführt. Zu den Nachuntersuchungen/ Therapien gehörten: Endoskopie $(n=19)$, Chirurgie $(\mathrm{n}=11)$ und Medikamente $(\mathrm{n}=50)$ (Pennazio et al. 2004).

Die Ben Soussan Studie wies hingegen mit einem Wert von 37\% (13/35) einen geringeren therapeutischen Impact bei den Blutungspatienten auf. Folgende Nachuntersuchungen/ Therapien wurden vorgenommen: Endoskopie $(n=10)$, Chirurgie $(n=2)$ und medikamentöse Behandlungen $(\mathrm{n}=1)$ (Ben Soussan et al. 2004).

Von den Göttinger Patienten mit unklarer Anämie bekamen 69,6\% (16/23) eine Therapie: Chirurgie $(\mathrm{n}=1)$ sowie Medikamente $(\mathrm{n}=15)$.

In der Studie von Apostolopoulos konnte bei 48\% (14/29) der Patienten mit unklarer Anämie im Anschluss an die VKE eine gezielte Therapie vorgenommen werden: Endoskopien mit APCLaser bei Angiodysplasien $(n=4)$, Endoskopie mit Polypektomie $(n=2)$, chirurgische Entfernung von Jejunaltumoren $(\mathrm{n}=2)$, medikamentöse Behandlung mit Mesalazin und/ oder Kortikosteroiden bei Ulzerationen im Dünndarm $(n=6)$, medikamentöse Eisensubstitution $(n=13)$. Im späteren Verlauf wurde bei zwei der vier Patienten, welche eine Therapie mit APCLaser erhielten, eine chirurgische Intervention vollzogen (Apostolopoulos et al. 2006).

Von den drei Göttinger Dünndarmtumorpatienten wurde bei zwei Patienten mit Jejunummetastasen, eine Jejunumsegmentresektion in palliativer Absicht durchgeführt. Ein abwartendes Verhalten wurde bei der Lymphom-Patientin eingenommen.

In Baileys Studie wurde bei 23 von 26 Patienten der Tumor chirurgisch reseziert, bei zwölf Patienten (52\%) mit kurativem Ansatz (Bailey et al. 2006).

Rondonottis Tumorpatienten $(n=124)$ erhielten $\mathrm{zu}$ 95\% eine chirurgische Tumorresektion (Rondonotti et al. 2008). 


\subsection{Tabellarischer Vergleich der verschiedenen Studien}

\begin{tabular}{|c|c|c|c|c|}
\hline Autoren der Studie & Zeitraum & Wo durchgeführt? & Anzahl der VKE & Altersdurchschnitt der Patienten \\
\hline Eigene Studie 2014 & 2006-2010 & Göttingen (Deutschland) & 203 & $58( \pm 19,5) \mathrm{J}$. \\
\hline Rondonotti et al. 2010 & 2001-2008 & Lombardei (Italien) & 2921 & k.A. \\
\hline Liao et al. 2010 & $2000-2008$ & Zusammenfassung von 227 Studien & 22840 & k.A. \\
\hline Toy et al. 2008 & 2003-2005 & Kalifornien (USA) & 145 & $55,7 \mathrm{~J}$ \\
\hline Triantafyllou et al. 2007 & $2002-2006$ & Athen (Griechenland) & 87 & $62( \pm 11,8) \mathrm{J}$ \\
\hline Cheon et al. 2007 & $2002-2006$ & Korea & 1291 & k.A. \\
\hline Pennazio et al. 2004 & 2001-2002 & Turin, Mailand (Italien) & 100 & $63 \mathrm{~J}$. \\
\hline
\end{tabular}

\begin{tabular}{l|l|l|l|} 
Autoren der Studie & Anzahl Männer/ Frauen & Diabetes mellitus-Patienten & BMI-Median der Patienten \\
\hline Eigene Studie 2014 & $118(58 \%) / 85(42 \%)$ & $16,8 \%(\mathrm{n}=29)$ & 24,8 \\
\hline Rondonotti et al. 2010 & k.A. & k.A. & k.A. \\
\hline Liao et al. 2010 & k.A. & k.A. & k.A. \\
Toy et al. 2008 & $61(41 \%) / 85(59 \%)$ & k.A. & k.A. \\
\hline Triantafyllou et al. 2007 & $36(41,4 \%) / 51(58,6 \%)$ & $33 \%(\mathrm{n}=29)$ & k.A. \\
\hline Cheon et al. 2007 & k.A. & k.A. & k.A. \\
\hline Pennazio et al. 2004 & $56(56 \%) / 44(44 \%)$ & k.A. & k.A.
\end{tabular}

\begin{tabular}{|c|c|c|}
\hline Autoren der Studie & 1. Indikation/ diagnostische Ausbeute & a) häufigster pathologischer Befund \\
\hline Eigene Studie 2014 & unklare GIB $(45,3 \%) / 80,4 \%$ & Schleimhautläsionen: $47,8 \%(n=44)$ \\
\hline Rondonotti et al. 2010 & unklare GIB $(43,3 \%) / 62,5 \%$ & k.A. \\
\hline Liao et al. 2010 & unklare GIB $(66 \%) / 60,5 \%$ & Angiodysplasien: $50 \%(\mathrm{n}=1375)$ \\
\hline Toy et al. 2008 & unklare sichtbare GIB $(38 \%) / 65 \%$ & arteriovenöse Malformation: $41 \%(\mathrm{n}=19)$ \\
\hline Triantafyllou et al. 2007 & Eisenmangelanämie $(58,6 \%) / \mathrm{k} . \mathrm{A}$. & k.A. \\
\hline Cheon et al. 2007 & unklare GIB $(48,7 \%) /$ k.A & k.A. \\
\hline Pennazio et al. 2004 & unklare GIB $(100 \%) / 47 \%$ & Angiodysplasien: $29 \%(\mathrm{n}=29)$ \\
\hline
\end{tabular}

\begin{tabular}{|c|c|c|}
\hline Autoren der Studie & 2. Indikation/ diagnostische Ausbeute & 3. Indikation/ diagnostische Ausbeute \\
\hline Eigene Studie 2014 & unklare abdominelle Schmerzen $(24 \%) / 40,8 \%$ & unklare Anämie $(11,3 \%) / 78 \%$ \\
\hline Rondonotti et al. 2010 & unklare Anämie $(23,9 \%) / 40,9 \%$ & V.a. M. Crohn $(7,8 \%) / 47,3 \%$ \\
\hline Liao et al. 2010 & klinische Symptome $(10,6 \%) /$ k.A. & M.Crohn (10,4\%)/ 55,3\% \\
\hline Toy et al. 2008 & okkulte GIB $(22 \%) / 56 \%$ & unklare abdominelle Schmerzen (20\%)/ 46\% \\
\hline Triantafyllou et al. 2007 & unklare Diarrhoe $(20,7 \%) /$ k.A. & rezidivierender Teerstuhl $(13,7 \%) / \mathrm{k} . \mathrm{A}$. \\
\hline Cheon et al. 2007 & unklare abdominelle Schmerzen $(17,7 \%) /$ k.A. & andere Symptome $(13,5 \%) /$ k.A. \\
\hline Pennazio et al. 2004 & ------ & ----- \\
\hline
\end{tabular}




\begin{tabular}{|c|c|c|}
\hline Autoren der Studie & 4. Indikation/ diagnostische Ausbeute & 5. Indikation/ diagnostische Ausbeute \\
\hline Eigene Studie 2014 & unklare Diarrhoe $(6,4 \%) / 46 \%$ & Polyp- und Tumor-Suche $(5,4 \%) / 90,9 \%$ \\
\hline Rondonotti et al. 2010 & unklare abdominelle Schmerzen $(5,3 \%) / 14,9 \%$ & chronische Diarrhoe $(4,8 \%) / 27,4 \%$ \\
\hline Liao et al. 2010 & andere Symptome (7\%)/ k.A. & Polyp- und Tumorsuche $(3,5 \%) / 55,9 \%$ \\
\hline Toy et al. 2008 & M. Crohn $(12 \%) / 61,5 \%$ & Eisenmangelanämie $(2,7 \%) / 0 \%$ \\
\hline Triantafyllou et al. 2007 & unklare abdominelle Schmerzen $(11,5 \%) /$ k.A. & andere Symptome $(9,2 \%) /$ k.A. \\
\hline Cheon et al. 2007 & unklare Eisenmangelanämie $(6,4 \%) /$ k.A. & chronische Diarrhoe $(4,5 \%) /$ k.A. \\
\hline Pennazio et al. 2004 & ----- & ------ \\
\hline
\end{tabular}

\begin{tabular}{|l|l|l|} 
Autoren der Studie & häufigster pathologischer Befund insgesamt & Erreichen des Zökums \\
\hline Eigene Studie 2014 & Schleimhautläsionen: $75,7 \%(\mathrm{n}=131)$ & $83,7 \%(\mathrm{n}=170)$ \\
\hline Rondonotti et al. 2010 & Angiodysplasien: $52,2 \%(771 / 1477)$ & $81,2 \%(\mathrm{n}=2371)$ \\
\hline Liao et al. 2010 & k.A. & $83,5 \%(\mathrm{n}=9905)$ \\
\hline Toy et al. 2008 & arteriovenöse Malformation: 24\% $(\mathrm{n}=35)$ & $80 \%(\mathrm{n}=116)$ \\
\hline Triantafyllou et al. 2007 & Schleimhautläsionen: $24 \%(\mathrm{n}=21)$ & $82,8 \%(\mathrm{n}=72)$ \\
\hline Cheon et al. 2007 & k.A. & k.A. \\
\hline Pennazio et al. 2004 & Angiodysplasien: $21 \%(\mathrm{n}=21)$ & $79 \%(\mathrm{n}=79)$
\end{tabular}

\begin{tabular}{|c|c|c|}
\hline Autoren der Studie & Magenpassagezeiten & Magenpassage ohne Diabetes mellitus/mit D.m. \\
\hline Eigene Studie 2014 & 1-462 Min. $(\bar{x}=21$ Min. $)$ & 1-462 Min. $(\bar{x}=22$ Min. $) / 1-139$ Min. $(\bar{x}=13$ Min. $)$ \\
\hline Rondonotti et al. 2010 & k.A. & k.A. \\
\hline Liao et al. 2010 & k.A. & k.A. \\
\hline Toy et al. 2008 & k.A. & k.A. \\
\hline Triantafyllou et al. 2007 & k.A. & 4-108 Min. $(\bar{X}=24) / 1-478$ Min. $(\bar{X}=87)$ \\
\hline Cheon et al. 2007 & k.A. & k.A. \\
\hline Pennazio et al. 2004 & k.A. & k.A. \\
\hline
\end{tabular}

\begin{tabular}{|c|c|c|}
\hline Autoren der Studie & Dünndarmpassagezeiten & Dünndarmpassage ohne Diabetes mellitus/ mit D.m. \\
\hline Eigene Studie 2014 & 18-522 Min. $(\bar{X}=245$ Min. $)$ & 48-525 Min. $(\bar{x}=255$ Min. $) / 15-470$ Min. $(\bar{x}=216$ Min. $)$ \\
\hline Rondonotti et al. 2010 & k.A. & k.A. \\
\hline Liao et al. 2010 & k.A. & k.A. \\
\hline Toy et al. 2008 & $\bar{x}=216$ Min. & k.A. \\
\hline Triantafyllou et al. 2007 & k.A. & $302 \pm 62,7$ Min. / 261,2 \pm 55,5 Min. \\
\hline Cheon et al. 2007 & k.A. & k.A. \\
\hline Pennazio et al. 2004 & k.A. & k.A. \\
\hline
\end{tabular}




\begin{tabular}{|l|l|l|l|} 
Autoren der Studie & Kapselretention & Kapselretention: häufigste Ursache & a) natürliche Exkretion \\
\hline Eigene Studie $\mathbf{2 0 1 4}$ & $2 \%(\mathrm{n}=4)$ & M. Crohn: $1,5 \%(\mathrm{n}=3)$ & $0,5 \%(\mathrm{n}=1)$ \\
\hline Rondonotti et al. $\mathbf{2 0 1 0}$ & $2,1 \%(\mathrm{n}=61)$ & unklare GIB: $75,8 \%(15 / 29)$ & $1,1 \%(\mathrm{n}=32)$ \\
\hline Liao et al. 2010 & $1,4 \%(\mathrm{n}=184)$ & M.Crohn: $35,3 \%(\mathrm{n}=48)$ & $15,8 \%(\mathrm{n}=$ k.A. $)$ \\
\hline Toy et al. 2008 & $4,1 \%(\mathrm{n}=6)$ & unklare abdominelle Schmerzen: $2 \%(\mathrm{n}=3)$ & $0,7 \%(\mathrm{n}=1)$ \\
\hline Triantafyllou et al. 2007 & k.A. & k.A. & k.A. \\
\hline Cheon et al. $\mathbf{2 0 0 7}$ & $2,5 \%(\mathrm{n}=32)$ & M. Crohn: $13 \%(3 / 23)$ & $34 \%(11 / 32)$ \\
\hline Pennazio et al. $\mathbf{2 0 0 4}$ & $5 \%(\mathrm{n}=5)$ & M. Crohn: $2 \%(\mathrm{n}=2)$ & $0 \%$
\end{tabular}

\begin{tabular}{|l|l|l|l|} 
Autoren der Studie & b) endoskopische Bergung & c) chirurgische Bergung & d) akute Obstruktion durch VKE \\
\hline Eigene Studie 2014 & $1 \%(\mathrm{n}=2)$ & $0,5 \%(\mathrm{n}=1)$ & $0,5 \%(\mathrm{n}=1)$ \\
\hline Rondonotti et al. $\mathbf{2 0 1 0}$ & $0,14 \%(\mathrm{n}=4)$ & $0,75 \%(\mathrm{n}=22)$ & $0,07 \%(\mathrm{n}=2)$ \\
\hline Liao et al. 2010 & $12,5 \%(\mathrm{n}=$ k.A.) & $58,7 \%(\mathrm{n}=108)$ & k.A. \\
\hline Toy et al. 2008 & $0,7 \%(\mathrm{n}=1)$ & $2,7 \%(\mathrm{n}=4)$ & $0 \%$ \\
\hline Triantafyllou et al. 2007 & k.A. & k.A. & k.A. \\
\hline Cheon et al. 2007 & $12,5 \%(4 / 32)$ & $53 \%(17 / 32)$ & $15,6 \%(5 / 32)$ \\
\hline Pennazio et al. $\mathbf{2 0 0 4}$ & $1 \%(\mathrm{n}=1)$ & $4 \%(\mathrm{n}=4)$ & $0 \%$
\end{tabular}

\begin{tabular}{l|l|l|l|l|} 
Autoren der Studie & Therapie & a) medikamentöse Therapie & b) Endoskopie mit Intervention & c) operative Therapie \\
\hline Eigene Studie 2014 & $73,4 \%(\mathrm{n}=149)$ & $66 \%(\mathrm{n}=133)$ & $4 \%(\mathrm{n}=8)$ & $4,4 \%(\mathrm{n}=9)$ \\
\hline Rondonotti et al. 2010 & k.A. & k.A. & k.A. & k.A. \\
\hline Liao et al. 2010 & k.A. & k.A. & k.A. & k.A. \\
\hline Toy et al. 2008 & $61 \%(46 / 75)$ & k.A. & k.A. & k.A. \\
\hline Triantafyllou et al. 2007 & k.A. & k.A. & k.A. & k.A. \\
\hline Cheon et al. 2007 & k.A. & k.A. & $20 \%(19 / 91)$ & k.A. \\
\hline Pennazio et al. 2004 & $88 \%(80 / 91)$ & $55 \%(50 / 91)$ & $12 \%(11 / 91)$
\end{tabular}

\begin{tabular}{|l|l|}
\hline \multicolumn{2}{|l|}{ Abkürzungen: } \\
\hline BMI = Body-Mass-Index & Min. = Minute \\
D.m. = Diabetes mellitus & $\mathrm{n}=$ Anzahl \\
GIB = gastrointestinale Blutung & V.a. = Verdacht auf \\
J. = Jahre & VKE = Videokapselendoskopie \\
k.A. = keine Angaben & $\bar{x}=$ Median \\
M. = Morbus & \\
\hline
\end{tabular}




\subsection{Vergleich der Kapselendoskopie mit anderen Untersuchungsmethoden}

\subsubsection{Vergleich mit der Push-Enteroskopie}

Vergleichende Studien zeigten, dass die Kapselendoskopie bei Patienten mit einer obskuren gastrointestinalen Blutung oder anderen Dünndarmerkrankungen doppelt so viele Läsionen detektieren konnte wie die Push-Enteroskopie (Ell et al. 2002; Lewis und Swain 2002; Mylonaki et al. 2003). Da die Push-Enteroskopie nur das Jejunum erreichen kann, ist die VKE dieser Enteroskopie deutlich überlegen (May et al. 2010).

Die diagnostische Ausbeute in der Arbeit von Lewis über die Abklärung der obskuren GIBlutung lag bei der Push-Enteroskopie bei 30\% (6/20), bei der VKE hingegen bei 55\% (11/20). Dieser Unterschied war nicht signifikant $(\mathrm{p}=0,0625)$. Jedoch bevorzugten die Patienten die VKE gegenüber der Enteroskopie wegen des besseren Untersuchungskomforts (Lewis und Swain 2002). Die Arbeit von Mylonaki zeigte bei der Detektion von unklaren Blutungen eine signifikante Ausbeute $(\mathrm{p}<0,05)$ der VKE von 68\% (34/50) und der Enteroskopie von 32\% (16/50) (Mylonaki et al. 2003).

Die Metaanalyse von Triester et al. verglich in 14 Studien $(n=396$ VKE) die diagnostischen Ausbeuten der VKE und der Push-Enteroskopie bei der Indikation unklare gastrointestinale Blutung. Die Ausbeute der VKE lag bei 63\%, die der Push-Enteroskopie bei 28\% (p < 0,00001). Bei klinisch signifikanten Ergebnissen $(n=376)$ wies die VKE eine Ausbeute von $56 \%$ auf, die Push-Enteroskopie hingegen 26\% ( $\mathrm{p}<0,00001)$. Zehn der 14 Studien, die die VKE mit der Push-Enteroskopie verglichen, wiesen nach, dass die VKE zu 36\% vaskuläre Läsionen aufzeigte, die Enteroskopie demgegenüber nur 20\% ( $\mathrm{p}=0$,00001). Entzündliche Läsionen konnten in der VKE zu 11\% gesehen werden, in der Push-Enteroskopie nur zu 2\% (p=0,00001). Kein signifikanter Unterschied zwischen den beiden Untersuchungen konnte bei der Tumorsuche oder anderen Indikationen gefunden werden (Triester et al. 2005).

In der Studie von Pennazio $(n=100)$ wiesen die Kapselendoskopie und die Push-Enteroskopie eine gesamte diagnostische Ausbeute von 67\% (33/51) auf. Zusammengefasst konnte die PushEnteroskopie eine Blutungsursache bei 29\% der Patienten (15/51), die VKE bei 59\% der Patienten (30/51) finden (Pennazio et al. 2004).

Nur 10,8\% (146/1349) der VKE-Untersuchungen in Lewis' Metaanalyse ( $n=1349)$ ergaben falsch-negative Befunde. Die Rate falsch-negativer Befunde der vergleichbaren Methoden (Push-Enteroskopie, Magendarmpassage, Ileo- und Koloskopien) lag bei 73\% (989/1349). Diese 
Untersuchungen konnten in 68,7\% (253/368) keine vaskulären Läsionen detektieren. Bei Anwendung der VKE konnten hingegen lediglich 5,9\% (22/368) der Läsionen nicht entdeckt werden. Von 169 Ulzerationen fehlten 0,5\% (8/169) in den VKE-Befunden, 78,7\% (133/169) der Ulzerationen wurden in den anderen Untersuchungsergebnissen nicht gesehen. Neoplasien wurden in 18,9\% (20/106) der Fälle nicht durch die VKE entdeckt, wohingegen die vergleichbaren Methoden zu 63,2\% (67/106) die Tumoren nicht darstellen konnten. Die exakte Rate der diagnostischen Ausbeute der Push-Enteroskopie im Vergleich zur VKE lag bei 14,8\% (77/519) vs. 85,2\% (442/519) (Lewis et al. 2005).

$\mathrm{Da}$ in der gastroenterologischen Abteilung in Göttingen bisher kein direkter Vergleich zwischen der VKE und der Push-Enteroskopie oder anderer folgenden Untersuchungen vorliegt, können wir über die Wertigkeit der Videokapsel im direkten Vergleich zu anderen Dünndarmuntersuchungsmethoden keine Aussage machen.

\subsubsection{Vergleich mit der Doppelballonenteroskopie}

Bisher wurden in der Literatur mehrere Arbeiten, die die VKE mit der Doppelballonenteroskopie (DBE) vergleichen, veröffentlicht. Nakamura et al. verglichen die diagnostische Ausbeute von Kapselendoskopie und DBE $(n=32$ wurden mit der Kapsel untersucht, $n=28$ mit beiden Methoden). Die diagnostische Ausbeute der VKE lag bei 59,4\% (19/32), die der DBE bei 42,9\% (12/28). Bei der Kapselendoskopie war die Rate der kompletten Dünndarmuntersuchung signifikant höher (90,6 vs. 62,5\%, p < 0,05) (Nakamura et al. 2006).

Marmo zeigte in seiner Studie $(n=193)$, dass die VKE in 34 Fällen eine Dünndarmblutung nachweisen konnte. Bei 12 dieser 34 Blutungspatienten konnte in der DBE keine pathologische Auffälligkeit gefunden werden. Bei den restlichen 22 Blutungspatienten detektierte die DBE eine Pathologie (vaskuläre Läsionen, Neoplasien, Ulzerationen und Divertikel) (Marmo et al. 2009). Bei der Detektion vaskulärer $[38,3 \%(n=74)$ vs. $37,3 \%(n=72)]$ und entzündlicher Läsionen [8,8\% $(\mathrm{n}=17)$ vs. 6,2\% $(\mathrm{n}=12)]$ lieferten beide Untersuchungen (VKE vs. DBE) etwa gleiche Ergebnisse, wohingegen die DBE häufiger Polypen und Neoplasien aufdecken konnte [10,4\% $(n=20)$ vs. $15,5 \%(n=30)]$ (Marmo et al. 2009).

Pashas Metaanalyse in den USA stellte elf Studien mit insgesamt 397 Patienten gegenüber, welche die VKE mit der Doppelballonendoskopie verglichen. Die VKE erreichte eine diagnostische Ausbeute von 60\% (238/397), die DBE eine von 57\% (205/360). Beide Untersuchungsmethoden erzielten im Gegensatz zur Marmo-Studie gleiche pathologische Ergebnisse 
bezüglich vaskulärer Läsionen [24\% $(\mathrm{n}=371)$ vs. 24\% $(\mathrm{n}=364)$ ], entzündlicher Veränderungen $[18 \%(\mathrm{n}=343)$ vs. $16 \%(\mathrm{n}=336)]$ und Polyp- und Tumorsuche $[11 \%(\mathrm{n}=343)$ vs. $11 \%$ $(n=336)$ ]. Pasha favorisiert deshalb, die nicht-invasive Videokapselendoskopie bereits bei den initialen diagnostischen Maßnahmen einzusetzen, da sie potentiell den gesamten Dünndarm visualisieren kann. Die VKE gewährleistet eine gute Determinierung der pathologischen Veränderungen für die DBE. Die anschließende DBE besitzt darüber hinaus die Option, die detektierten Veränderungen diagnostisch durch eine Biopsie histologisch abzuklären bzw. therapeutisch tätig zu werden (Pasha et al. 2008).

Auch die DBE kann wie die VKE zu Komplikationen führen. Nach Mensinks Multicenterstudie $(\mathrm{n}=2362 \mathrm{DBE})$ lag die Gesamt-Komplikationsrate bei 1,7\% (40/2362). Bei diagnostischen Eingriffen der DBE kam es in 0,8\% (13/1728) und bei therapeutischen Eingriffen in 4,3\% (27/634) zu Komplikationen. Zu den Hauptkomplikationen zählten Blutungen, Pankreatitis sowie Perforationen (Mensink et al. 2007).

Die DGVS sieht in ihrem Update von 2010 die VKE und die DBE als sich ergänzende und nicht konkurrierende Untersuchungsmethoden (May et al. 2010).

\subsubsection{Vergleich mit Enteroklysma-Untersuchungen}

In der Studie von Apostolopoulos $(\mathrm{n}=51)$ erreichte die VKE eine diagnostische Ausbeute von 57\% (29/51) im Vergleich zu 11,8\% (6/51) bei der Enteroklysma-Untersuchung. Zudem konnten durch die VKE Angiodysplasien, Ulzerationen, diffuse Erosionen und Polypen gesehen werden, welche durch die vergleichende Untersuchung zum Teil nicht gesehen wurden. Hingegen waren sämtliche Befunde, die durch das Enteroklysma detektiert wurden, auch durch die VKE sichtbar (Apostolopoulos et al. 2006).

In Voderholzers Studie $(\mathrm{n}=22)$ gelang der Vergleich zwischen der VKE und der CT-Enteroklysma-Untersuchung. Die diagnostische Ausbeute der VKE war um 23\% höher [59\% (18/22) vs. 36\% (8/22)] als die der CT-Sellink-Untersuchung $(\mathrm{p}=0,12)$ (Voderholzer et al. 2003). In einer anderen Studie desselben Autors $(n=41)$ zwei Jahre später konnten bei 25 Patienten jejunale oder ileale Läsionen in der VKE nachgewiesen werden, wohingegen das CT-Enteroklysma nur bei zwölf Patienten Veränderungen im Dünndarm fand (Voderholzer et al. 2005).

Gölders Studie $(n=36)$, die für unterschiedliche Indikationen die VKE mit dem MRTEnteroklysma verglich, zeigte einen signifikanten Unterschied in der diagnostischen Ausbeute von 36\% (5/14) bei der Abklärung von unklaren gastrointestinalen Blutungen $(n=14)$ hin- 
sichtlich der VKE $(p=0,007)$. Die VKE konnte zudem mehr signifikante Läsionen $(p=0,016)$ bei Patienten mit M. Crohn $(\mathrm{n}=18)$ in den ersten zwei Dünndarmabschnitten nachweisen als das MRT-Enteroklysma (Gölder et al. 2006).

\subsubsection{Vergleich mit der Angiografie/ CT-Abdomen}

Die Studie von Saperas et al. $(n=25)$ stellte die VKE sowohl der CT-Angiografie als auch der Standardangiografie gegenüber. Demnach konnte die Blutungsursache bei der VKE zu 72\% (18/25), bei der CT-Angiografie zu 24\% (6/25) und bei der Standardangiografie zu 56\% (14/25) herausgefunden werden (Saperas et al. 2007).

May et al. erklärten sich diese Unterschiede dadurch, dass eine nötige Blutungsstärke für die Angiografie vorhanden sein muss, um eine floride Blutung detektieren zu können. Bei starken Blutungen besitzt die Angiografie den Vorteil, dass dabei durch Embolisation eine Blutstillung vorgenommen werden kann (May et al. 2010).

In Eliakims Arbeit $(\mathrm{n}=35)$ wurde bei M. Crohn-Patienten eine signifikante diagnostische Ausbeute von $77 \%$ in der VKE nachgewiesen, im Gegensatz zu $20 \%$ bei der CT-AbdomenUntersuchung ( $\mathrm{p}<0,05)$ (Eliakim et al. 2004).

\subsubsection{Vergleich mit der Magen-Darm-Passage}

Einen Vergleich zwischen der VKE und bildgebenden Verfahren stellte Triesters Metaanalyse her. Anhand von drei Studien $(n=88)$ wurde die diagnostische Ausbeute der VKE und der Magen-Darm-Passage miteinander verglichen: die Ausbeute der VKE lag bei 67\%, die der Barium-Kontrastmittel-Darstellung bei $8 \%$ ( $p<0,00001)$. Klinisch signifikante Resultate konnten bei der VKE zu 42\% gezeigt werden, bei der Magen-Darm-Passage zu 6\% (Triester et al. 2005). Eine große Differenz der diagnostischen Ausbeute wies die VKE im Gegensatz zur Magen-Darm-Passage [77\% vs. 23\% (p<0,05)] auch in der Studie von Eliakim $(\mathrm{n}=35)$ auf (Eliakim et al. 2004). Ebenfalls wurde in Dubcencos Studie $(n=45)$ die VKE der radiografischen Dünndarmpassage gegenüber gestellt. Hier erzielte die VKE nach histologischer Diagnose eine Sensitivität von 89,6\% (Spezifität $=100 \%, \quad P P V=100 \%, \quad N P V=77 \%$ ), die Dünndarmpassage eine Sensitivität von 27,6\% (Spezifität $=100 \%$, PPV $=100 \%, \mathrm{NNP}=32,3 \%$ ) (Dubcenco et al. 2005). Noch signifikanter war der Unterschied der diagnostischen Ausbeute in der Lewis-Studie. Die Magen-Darm-Passage detektierte zu 9,9\% (37/374) pathologische Auffälligkeiten und die VKE zu 90,1\% (337/374) (p < 0,0001) (Lewis et al. 2005). 


\subsubsection{Vergleich mit der intraoperativen Enteroskopie}

Hartmanns Studie $(n=47)$ stellte die Ergebnisse zwischen der VKE und dem früheren Goldstandard der Dünndarmdiagnostik - der intraoperativen Enteroskopie - gegenüber. Das Resultat erbrachte ähnliche diagnostische Ausbeuten: die VKE mit 74,4\%, die intraoperative Enteroskopie mit 72,3\%. Aufgrund der Mortalität der intraoperativen Enteroskopie von 2\% zieht Hartmann die VKE vor (Mortalität: 0\%) (Hartmann et al. 2005). 


\subsection{Ausblick: Zukunft der Dünndarmvideokapselendoskopie}

Die Dünndarmkapselendoskopie ist derzeit der Goldstandard in der Abklärung von Dünndarmerkrankungen (Liao et al. 2010). Jedoch ist diese Untersuchungsmethode nur für wenige ausgewählte Indikationen bestimmt. Im November 2010 wurde die Dünndarmkapselendoskopie durch den gemeinsamen Bundesausschuss für Ärzte und Krankenkassen (G-BA) für die obskure gastrointestinale Blutung in den GKV-Leistungskatalog aufgenommen. Es bleibt abzuwarten, dass auch andere Indikationen in den Katalog integriert werden, z.B. die unklare Eisenmangelanämie.

Damit die Kosten der Videokapseln minimiert werden können, existieren bereits Pilotstudien zur Kapselauswertung durch Assistenzpersonal (Bossa et al. 2006; Levinthal et al. 2003; Niv und Niv 2005).

Ein wesentlicher Fokus der Entwicklungen liegt in der Kapselsteuerung und somit auch in den Interventionsmöglichkeiten. Die Innovation in der Kapselsteuerung besteht darin, dass die Kapsel durch einen externen Magneten gesteuert wird. Dabei wird eine magnetische Hülle über eine normale Videokapsel gestülpt. Die zweite wesentliche Neuerung in der Kapselendoskopie besteht in Interventionsmöglichkeiten. In der sogenannten „Spinnenkapsel“, die ihren Namen ihren acht beinchenartigen Fortsätzen an der Kapsel verdankt, sind mechanische Systeme integriert. Diese ermöglichen die anterograde wie auch retrograde Fortbewegung. Hierzu sind zudem zwei elektromagnetische Motoren erforderlich (May et al. 2010).

Seit August 2013 ist die dritte Generation der Given Imaging® Dünndarmkapsel PillCam SB3® durch die FDA zugelassen. Sie verfügt über ein neues Softwaresystem und kann die Anzahl der Bilderfrequenz pro Sekunde von zwei bis sechs Bilder variieren, je nachdem wie schnell die Kapsel durch die Peristaltik bewegt wird (GivenImaging 2013). 


\section{Zusammenfassung}

Retrospektiv wurden für diese Arbeit die Videokapseluntersuchungen des Universitätsklinikums Göttingen im Zeitraum 2006 bis 2010 ausgewertet. In dieser Zeit wurden 203 Videokapseluntersuchungen durchgeführt, zu Beginn mit der PillCam M2A/SB $®$, später mit der PillCam SB2@ von Given Imaging®.

Unsere Klientel bestand aus 118 männlichen (58\%) und 85 weiblichen (42\%) Patienten. Der Altersdurchschnitt betrug $58( \pm 19,5)$ Jahre. Im Vorfeld der VKE erhielten alle 203 Patienten Laborkontrollen, bidirektionale Endoskopien sowie Abdomen-Sonografien. Ergänzend wurden bei 116 Patienten $(57,1 \%)$ weitere Untersuchungen veranlasst.

Der überwiegende Anteil der Patienten [93\% ( $\mathrm{n}=189)]$ nahm die Videokapsel selbstständig ein, ein geringer Anteil von 7\% $(n=14)$ bekam die VKE unter endoskopischer Sicht verabreicht.

Eine optimale Auswertung der Videokapseln konnte bei 145 Patienten (71,3\%) bei sehr guter Sauberkeit des Dünndarms erzielt werden.

Bei 51 Patienten $(25,2 \%)$ war wegen Verstuhlung oder Blutresten lediglich eine eingeschränkte Befundung möglich. Bei sieben Patienten (3,5\%) verhinderten technische Fehler, starke Verschmutzung sowie Magenretention der Kapseln eine Auswertung.

Die Indikationen für die VKE bei unserer Patientenklientel waren: unklare gastrointestinale Blutung bei 92 Patienten (45,3\%), unklare abdominelle Schmerzen bei 49 Patienten $(24,1 \%)$, unklare Anämie bei 23 Patienten (11,3\%), der V.a. Morbus Crohn bei acht Patienten (4\%), Komplikationen der Diagnose Morbus Crohn bei fünf Patienten (2,5\%), unklare Diarrhoe bei 13 Patienten (6,4\%), Polyp- und Tumorsuche bei elf Patienten $(5,4 \%)$, rezidivierendes unklares Erbrechen bei einem Patienten $(0,5 \%)$ und das Eiweißverlustsyndrom bei einem anderen Patienten $(0,5 \%)$.

Innerhalb der Aufzeichnungszeit von acht bis neun Stunden wurde die komplette Dünndarmpassage bei 170 Patienten $(83,7 \%)$ erreicht. Bei 33 Patienten $(16,3 \%)$ dauerte die Passage länger als die Aufzeichnungszeit.

Vier Patienten (2\%) zeigten die Komplikation Kapselretention. Drei Patienten von ihnen waren an M. Crohn erkrankt. Dreimal gelang die Bergung durch Darmlavage oder endoskopische Bergung bzw. durch eine Kombination beider Maßnahmen, nur bei einem Patienten musste die Kapsel notfallmäßig operativ entfernt werden. 
Bei 85,2\% unserer Patientenpopulation (172/203) wurden in den Videokapselendoskopien pathologische Befunde im Dünndarm detektiert. Bei ca. 15\% der Patienten (32/203) erbrachte die VKE kein Ergebnis.

Die höchste diagnostische Ausbeute ergab sich bei der Abklärung der unklaren gastrointestinalen Blutung mit 80,4\% (74/92) und bei der unklaren Anämie mit 78\% (18/23). Die VKE war auch bei der Abklärung einer Polyp- und Tumorsuche mit 90,9\% (10/11) und den CrohnPatienten bzw. bei Verdacht auf M. Crohn mit 69\% (9/13) erfolgreich.

Eine niedrigere diagnostische Ausbeute zeigte sich bei den unklaren abdominellen Schmerzen mit 40,8\% (20/49) sowie bei den unklaren Diarrhoen mit 46\% (6/13).

Als häufigste Ursache für die gastrointestinalen Blutungen bzw. die unklare Anämie wurden Schleimhautläsionen zu 42,6\% (76/115) gefunden. Selten wurden in unserer Studie Divertikelblutungen, polypoide Läsionen oder Biopsie-Nachblutungen gesehen.

Die VKE wurde bei unseren vier Tumorpatienten entweder im Rahmen der Tumornachsorge, bei Verdacht auf Dünndarmtumor in Voruntersuchungen bzw. einer unklaren gastrointestinalen Blutung durchgeführt und erbrachte in allen vier Fällen entsprechende pathologische Befunde. Nach der VKE-Durchführung erhielten 35\% der Patienten (71/203) Nachuntersuchungen zur weiteren Diagnostik. Bei rund drei Viertel [73\% (148/203)] unserer untersuchten Patienten ergaben sich aus der VKE therapeutische Maßnahmen, vorwiegend eine medikamentöse Therapie.

Für die VKE-Auswertung empfehlen wir einen entsprechenden zeitlichen Rahmen (ca. ein bis zwei Stunden). Vermutlich konnten wir deshalb im Vergleich zur Literatur eine diagnostische Ausbeute von 85\% mit resultierenden Therapien für den Patienten bei $73 \%$ aufweisen. Damit nimmt die Kapsel bei gezielter Indikationsstellung nach vorausgegangenen Untersuchungen des oberen und unteren Gastrointestinaltraktes eine feste Stellung in der Diagnostik des mittleren Gastrointestinaltraktes ein. 


\section{Fazit}

Unsere Ergebnisse zeigen, dass die Kapselendoskopie bei klarer Indikationsstellung unter Berücksichtigung von Anamnese und gezielten Voruntersuchungen einen großen Stellenwert in der Dünndarmdiagnostik hat. Ein großer Vorteil der VKE ist die nicht-invasive Methode mit fehlender Mortalität. In unserer Patientenklientel verstarb kein Patient an den Folgen der VKE. Auch in der Literatur ist hierbei kein Todesfall vermerkt.

Ein bedeutsamer diagnostischer Benefit ergibt sich bei der Abklärung unklarer gastrointestinaler Blutung bzw. unklarer Eisenmangel-Anämie und zur Ausbreitungs- und Verlaufsdiagnostik des M. Crohn, weniger zur Abklärung unklarer abdomineller Schmerzen und unklarer Diarrhoe. Bei gezielten Fragestellungen kann bei Tumor-Patienten die VKE hilfreich sein.

Wir favorisieren die VKE nach der Gastroskopie, Koloskopie und in der Regel UltraschallUntersuchung des Abdomens als diagnostische Maßnahme bei unklarer gastrointestinaler Blutung bzw. unklarer Eisenmangelanämie einzusetzen.

Die kosten- und zeitintensive Videokapselendoskopie sollte unter optimalen Bedingungen stattfinden, damit eine hohe diagnostische Ausbeute erreicht werden kann. Die Patienten sollten im aufklärenden Vorbereitungsgespräch auf die Wichtigkeit der Darmreinigung hingewiesen werden. In unserer Studie waren bei einer Vorbereitung mit einem oder zwei Liter PEG-Lösung bei rund einem Viertel der Patienten (53/203) wegen Stuhlverschmutzung bzw. großer Blutkoagel keine ausreichenden Ergebnisse zu erhalten. Anhand der Ergebnisse dieser Arbeit sollte, wie in der Literatur vorgeschlagen, die Vorbereitung mit zwei Liter PEG-Lösung und ggf. Entschäumer mit mindestens 12-stündiger Nahrungskarenz vorgenommen werden.

Da die Komplikationsrate mit M. Crohn bzw. nach NSAR-Einnahme höher ist, sollte bei diesen Patienten eine bildgebende Dünndarmdiagnostik mit CT- oder MR-Sellink zur Stenosedetektion im Dünndarm vorgeschaltet werden. Im Gegensatz $\mathrm{zu}$ radiologischen Bildgebungen des Dünndarms können bei der VKE die Schleimhäute und Gefäße beurteilt werden.

Die Videokapselendoskopie kann zur Kostenersparnis beitragen, z.B. durch kürzere Diagnostikzeiten, geringere Applikation von Blutprodukten sowie verkürzte Krankenhausverweildauer, wenn sie zeitnah bei anerkannten Indikationen eingesetzt wird. Einen ersten Anlaufpunkt zeigt die NOKA-Studie, in der die Videokapselendoskopie notfallmäßig nach einer negativen Gastroskopie bei akuter gastrointestinaler Blutung erfolgreich eingesetzt wird (Schlag et al. 2013). 
Weiterhin wäre es wünschenswert, in Darmzentren VKE-Schwerpunkte zu etablieren, damit mehr Patienten mit entsprechenden Indikationen in kürzerer Zeit spezifisch untersucht werden können.

Fortschritte durch verbesserte Kapseleigenschaften bleiben abzuwarten, besonders die Möglichkeit, mit der VKE eine Biopsie zu entnehmen und eine Blutstillung vorzunehmen, würde ihre Einsatzmöglichkeiten um ein Vielfaches erhöhen. 


\section{Abbildungsverzeichnis}

Abb. 1: PillCam SB 2® im Vergleich zu einer $1 €$-Münze..................................................... 24

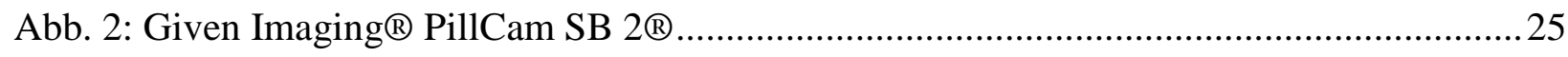

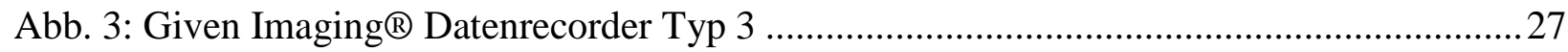

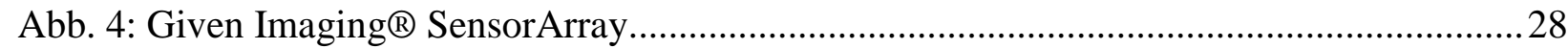

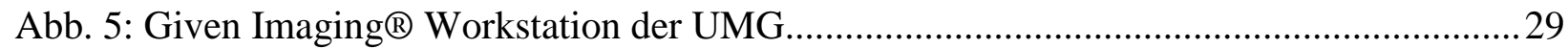

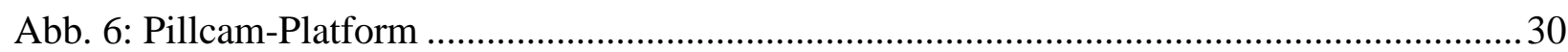

Abb. 7: Boxplot für Patientenalter, gruppiert nach Geschlecht ............................................... 37

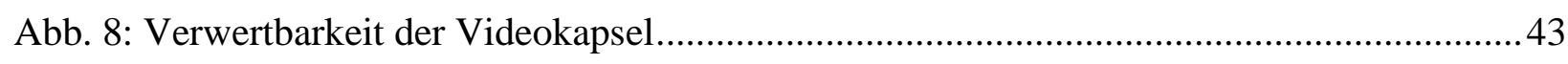

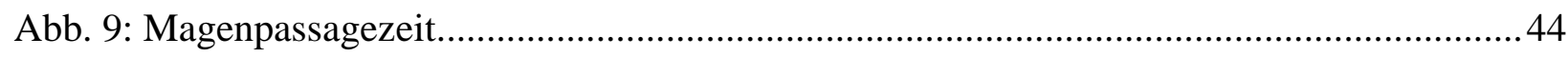

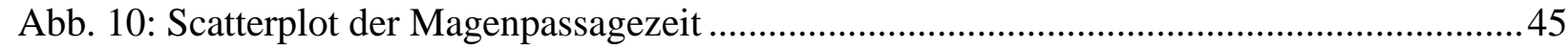

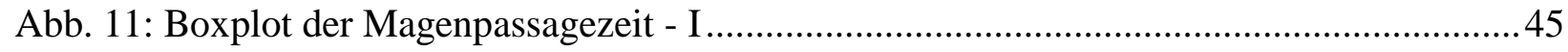

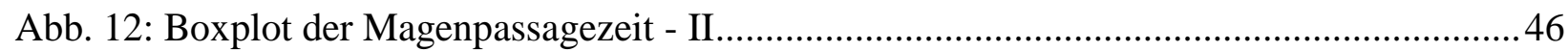

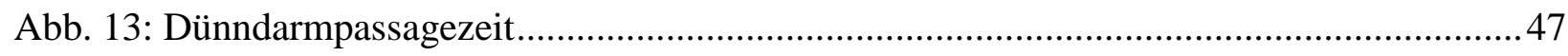

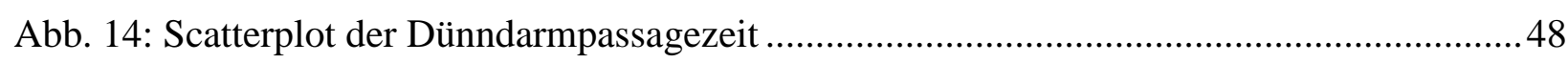

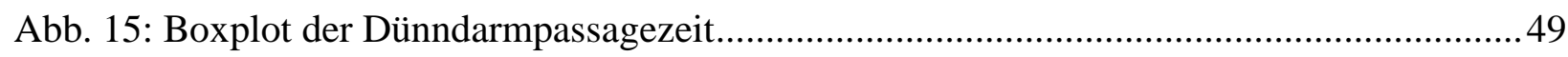

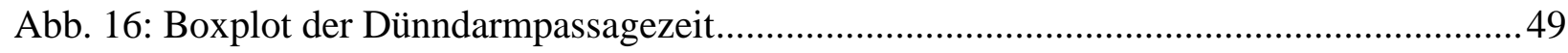

Abb. 17: VKE-Passage durch die Ileozökalklappe ................................................................ 51

Abb. 18: Nummerische Aufteilung der Schleimhautbefunde im Dünndarm..............................52

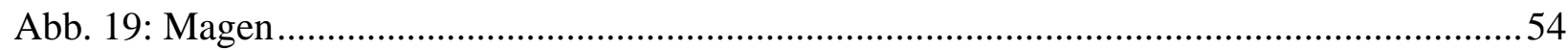

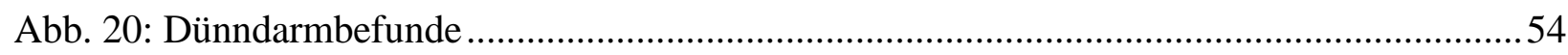

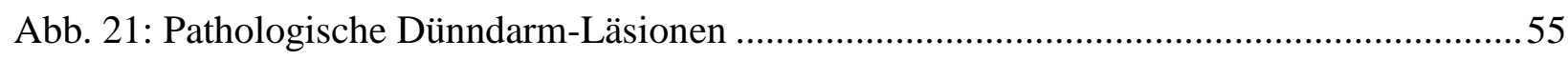

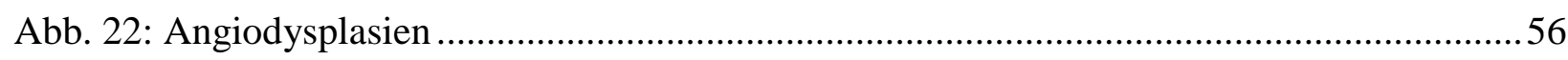

Abb. 23: Submuköse Erhabenheit mit Stenosierung.................................................................56

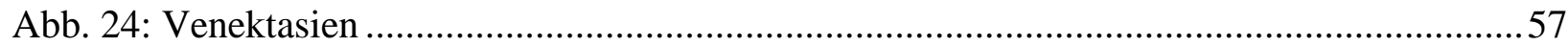

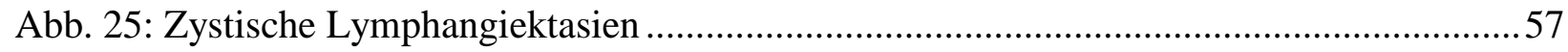

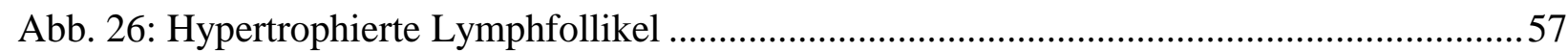

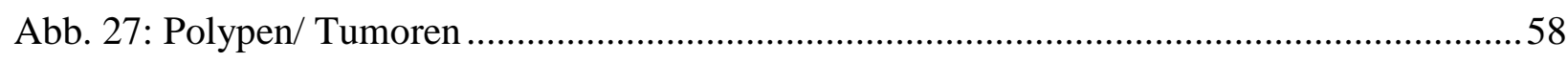

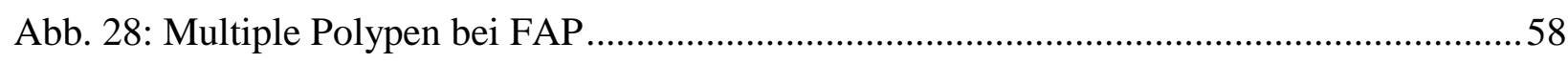

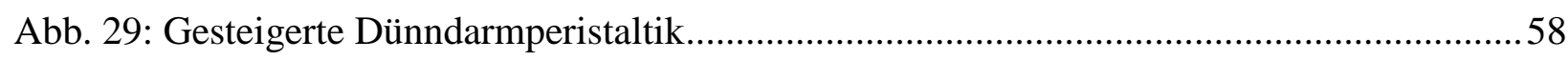

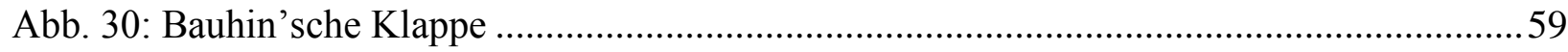


Abb. 31: Kuriose Befunde im Dünndarm .................................................................................. 59

Abb. 32: floride Dünndarmblutungen ohne erkennbare Blutungsquelle ...................................63

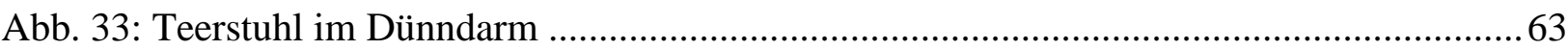

Abb. 34: M. Crohn Veränderungen - I ............................................................................. 68

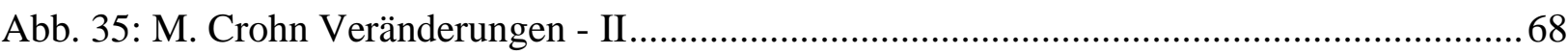

Abb. 36: Unterschiedliche Tumoren des Patientenkollektivs .................................................... 71

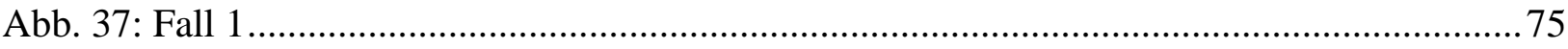

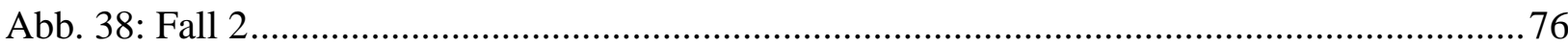

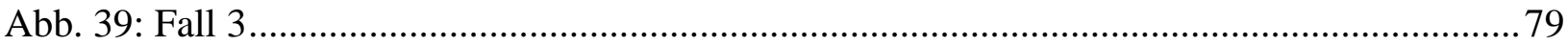

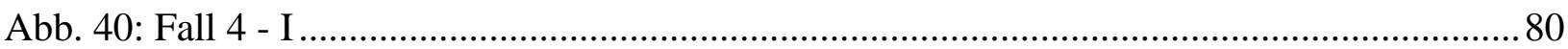

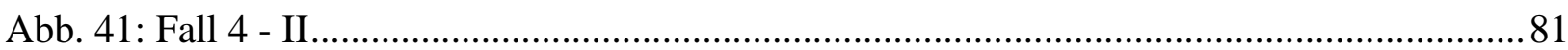

Abb. 42: Patientenanzahl der unterschiedlichen Nachuntersuchungen ...................................... 82 


\section{Tabellenverzeichnis}

Tabelle 1: Typische nicht pathologische VKE-Dünndarmkriterien........................................5

Tabelle 2: Altersabhängige Ursachen mittlerer gastrointestinaler Blutungen ............................. 8

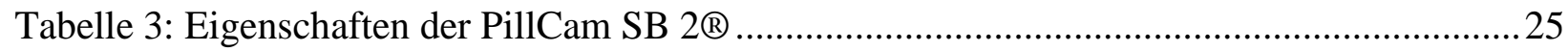

Tabelle 4: Aktuelle Indikationen der Dünndarm-Videokapselendoskopie ................................ 31

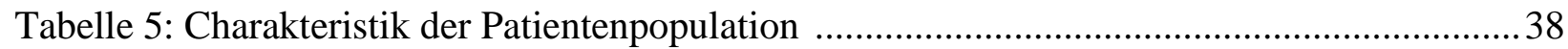

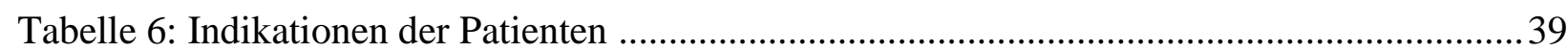

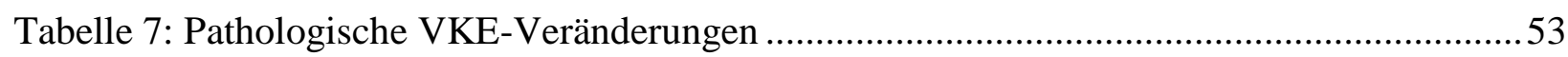

Tabelle 8: Befunde der VKE-Indikation unklare gastrointestinale Blutung ............................... 60

Tabelle 9: Befunde der VKE-Indikation unklare Anämie ...........................................................60

Tabelle 10: Befunde der VKE-Indikation unklare abdominelle Schmerzen ..............................61

Tabelle 11: Befunde der VKE-Indikation unklare Diarrhoe ..................................................61

Tabelle 12: Befunde der VKE-Indikation rezidivierendes Erbrechen \& Eiweißverlustsyndrom. 61

Tabelle 13: VKE-Befunde der Patienten mit floriden Dünndarmblutungen................................64

Tabelle 14: Häufigkeitsverteilung der Dünndarmblutungspatienten ........................................64

Tabelle 15: Anamnestische Angaben der gastrointestinalen Blutungspatienten ........................65

Tabelle 16: Dünndarmblutungen bei den unterschiedlichen BMI-Kategorien .......................... 66

Tabelle 17: Dünndarmveränderungen der Morbus Crohn- und V.a. M. Crohn-Patienten............69

Tabelle 18: Befunde der VKE-Indikation Polyp- und Tumorsuche .......................................... 72

Tabelle 19: Fallvorstellung 1: follikuläres B-Zell-Lymphom des Dünndarms ............................ 74

Tabelle 20: Fallvorstellung 2: VKE nach Jejunumsegmentresektion bei GIST ......................... 75

Tabelle 21: Fallvorstellung 3: Metastasen bei malignem Melanom im Jejunum ........................ 77

Tabelle 22: Fallvorstellung 4: Sarkommetastase im Jejunum................................................. 79

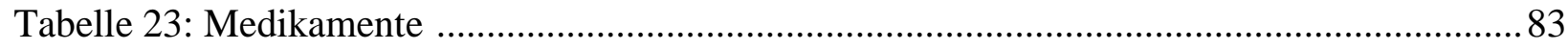




\section{Literaturverzeichnis}

Albert JG (2010): Update Kapselendoskopie - Einsatz bei Morbus Crohn. Endo heute 2 (23): 124-128.

Albert JG, Gobel CM, Lesske J, Lotterer E, Nietsch H, Fleig WE (2004): Simethicone for small bowel preparation for capsule endoscopy: a systematic, single-blinded, controlled study. Gastrointest Endosc 59(4): 487-491.

Albert JG, Wiedbrauck F, Keuchel M (2008): Small bowel endoscopy. Dtsch Med Wochenschr 133(21): 1136-1141.

Alkadhi H: Wie funktioniert CT?; hrsg. v. Alkadhi H, Leschka S, Stolzman P, Scheffel H; 1. Auflage; Springer-Verlag, Berlin, Heidelberg 2011.

Almeida N, Figueiredo P, Lopes S, Freire P, Lerias C, Gouveia H, Correia Leitao M (2008): Capsule endoscopy assisted by traditional upper endoscopy. Rev Esp Enferm Dig $\underline{100}(12): 758-763$.

Almeida N, Figueiredo P, Freire P, Lopes S, Lerias C, Gouveia H, Leitao MC (2010): The effect of metoclopramide in capsule enteroscopy. Dig Dis Sci 55(1): 153-157.

Apostolopoulos P, Liatsos C, Gralnek IM, Giannakoulopoulou E, Alexandrakis G, Kalantzis C, Gabriel P, Kalantzis N (2006): The role of wireless capsule endoscopy in investigating unexplained iron deficiency anemia after negative endoscopic evaluation of the upper and lower gastrointestinal tract. Endoscopy $\underline{38(11): 1127-1132 .}$

Apostolopoulos P, Kalantzis C, Gralnek IM, Liatsos C, Tsironis C, Kalantzis N (2008): Clinical trial: effectiveness of chewing-gum in accelerating capsule endoscopy transit time - a prospective randomized, controlled pilot study. Aliment Pharmacol Ther 28(4): 405-411.

Asakura H, Miura S, Morishita T, Aiso S, Tanaka T, Kitahora T, Tsuchiya M, Enomoto Y, Watanabe Y (1981): Endoscopic and histopathological study on primary and secondary intestinal lymphangiectasia. Dig Dis Sci 26(4): 312-320.

Bailey AA, Debinski HS, Appleyard MN, Remedios ML, Hooper JE, Walsh AJ, Selby WS (2006): Diagnosis and outcome of small bowel tumors found by capsule endoscopy: a three-center Australian experience. Am J Gastroenterol 101(10): 2237-2243.

Bandorski D, Irnich W, Brück M, Beyer N, Kramer W, Jakobs R (2008): Capsule endoscopy and cardiac pacemakers: investigation for possible interference. Endoscopy 40(1): 36-39.

Bandorski D, Stunder D, Höltgen R, Jakobs R, Keuchel M (2013): Kapselendoskopie bei Patienten mit Herzschrittmachern und implantierbaren Cardioverter-Defibrillatoren - Ist die formale Kontraindikation noch gerechtfertigt? Z Gastroenterol 51(08): 747-752.

Barkin JS, Frideman S (2002): Wireless capsule endoscopy requiring surgical intervention: the world's experience. Am J Gastroenterol 97: A83-.

Barkin JS, O'Loughlin C (2004): Capsule endoscopy contraindications: complications and how to avoid their occurrence. Gastrointest Endosc Clin N Am 14(1): 61-65.

Bartenstein P, Haug A, Hünerbein R: PET (Positronen-Emissions-Tomografie); in: Duale Reihe Radiologie, hrsg. v. Reiser M, Kuhn FP, Debus J; 3. Auflage; Georg Thieme Verlag KG, Stuttgart 2011, S. 143.

Barth C, Sinn I, Andus T (2007): Prognostische Bedeutung der Kapselendoskopie bei der mittleren intestinaler Blutung. Z Gastroenterol 45(08): P163.

Baumgart DC (2009): Diagnostik und Therapie von Morbus Crohn und Colitis ulcerosa. Dtsch Arztebl 106(8): 123-133. 
Ben Soussan E, Antonietti M, Herve S, Savoye G, Ramirez S, Lecleire S, Ducrotte P, Lerebours E (2004): Diagnostic yield and therapeutic implications of capsule endoscopy in obscure gastrointestinal bleeding. Gastroenterol Clin Biol 28(11): 1068-1073.

Bhattarai M, Bansal P, Khan Y (2013): Longest duration of retention of video capsule: A case report and literature review. World J Gastrointest Endosc $\underline{5}(7): 352-355$.

Biecker E, Heller J, Schmitz V, Lammert F, Sauerbruch T (2008): Effiziente Diagnostik und Therapie oberer gastrointestinaler Blutungen. Dtsch Arztebl International 105(5): 85-93.

Böcker W, Denk H, Heitz PU, Moch H: Idiopathische chronisch-entzündliche Darmerkrankungen; in: Repetitorium Pathologie; hrsg. v. Böcker W; 2. Auflage, Elsevier GmbH, Urban \& Fischer Verlag, München 2008, S. 303.

Böcker W, Höfler HK, Poremba C, Moll R, Tannapfel A, Probst-Hensch N: Nomenklatur der Tumoren; in: Pathologie; hrsg. v. Böcker W, Denk H, Heitz PU, Moch H; 4. Auflage, Elsevier GmbH, Urban \& Fischer Verlag, München 2008, S. 198.

Bossa F, Cocomazzi G, Valvano MR, Andriulli A, Annese V (2006): Detection of abnormal lesions recorded by capsule endoscopy. A prospective study comparing endoscopist's and

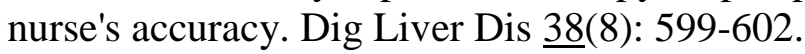

Braden B (2003): Akute untere Gastrointestinalblutung. Der Internist 44(5): 533.

Caddy GR, Moran L, Chong AK, Miller AM, Taylor AC, Desmond PV (2006): The effect of erythromycin on video capsule endoscopy intestinal-transit time. Gastrointest Endosc 63(2): 262-266.

Carey EJ, Heigh RI, Fleischer DE (2004): Endoscopic capsule endoscope delivery for patients with dysphagia, anatomical abnormalities, or gastroparesis. Gastrointest Endosc 59(3): 423-426.

Cave DR (2004): Reading wireless video capsule endoscopy. Gastrointest Endosc Clin N Am 14(1): 17-24.

Cave DR, Legnani P, de Franchis R, Lewis BS, ICCE (2005): ICCE consensus for capsule retention. Endoscopy 37(10): 1065-1067.

Cheifetz AS, Kornbluth AA, Legnani P, Schmelkin I, Brown A, Lichtiger S, Lewis BS (2006): The Risk of Retention of the Capsule Endoscope in Patients with Known or Suspected Crohn's Disease. Am J Gastroenterol 101(10): 2218-2222.

Cheifetz AS, Lewis BS (2006): Capsule endoscopy retention: is it a complication? J Clin Gastroenterol 40(8): 688-691.

Cheon JH, Kim YS, Lee IS, Chang DK, Ryu JK, Lee KJ, Moon JS, Park CH, Kim JO, Shim KN, et al. (2007): Can we predict spontaneous capsule passage after retention? A nationwide study to evaluate the incidence and clinical outcomes of capsule retention. Endoscopy 39(12): 1046-1052.

Cobrin GM, Pittman RH, Lewis BS (2006): Increased diagnostic yield of small bowel tumors with capsule endoscopy. Cancer 107(1): 22-27.

D'Halluin PN, Delvaux M, Lapalus MG, Sacher-Huvelin S, Ben Soussan E, Heyries L, Filoche B, Saurin JC, Gay G, Heresbach D (2005): Does the "Suspected Blood Indicator" improve the detection of bleeding lesions by capsule endoscopy? Gastrointest Endosc 61(2): 243-249.

Dai N, Gubler C, Hengstler P, Meyenberger C, Bauerfeind P (2005): Improved capsule endoscopy after bowel preparation. Gastrointest Endosc 61(1): 28-31.

Delorme S, Debus J: Morbus Crohn und Colitis ulcerosa; in: Duale Reihe Sonographie; hrsg. v. Delorme S, Debus J; 2. Auflage; Georg Thieme Verlag KG, Stuttgart 2005, S. 355. 
Delvaux M, Fassler I, Gay G (2004): Clinical usefulness of the endoscopic video capsule as the initial intestinal investigation in patients with obscure digestive bleeding: validation of a diagnostic strategy based on the patient outcome after 12 months. Endoscopy $\underline{36}(12)$ : 1067-1073.

Dubcenco E, Jeejeebhoy KN, Petroniene R, Tang SJ, Zalev AH, Gardiner GW, Baker JP (2005): Capsule endoscopy findings in patients with established and suspected small-bowel Crohn's disease: correlation with radiologic, endoscopic, and histologic findings. Gastrointest Endosc 62(4): 538-544.

Egger L, Fikentscher T, Poschenrieder F, Röhrl S, Friedrich C, Schill G, Agha A, Hoffstetter P, Überbacher C, Stroszczynski C, et al. (2012): Klinischer Stellenwert der Durchleuchtung der Magen-Darm-Passage (MDP) an einem Universitätsklinikum. Fortschr Röntgenstr 184(10): 893-898.

Eliakim R, Suissa A, Yassin K, Katz D, Fischer D (2004): Wireless capsule video endoscopy compared to barium follow-through and computerised tomography in patients with suspected Crohn's disease--final report. Dig Liver Dis 36(8): 519-522.

Elias G, Toubia N (2009): Safety of Capsule Endoscopy in the Setting of Implanted Cardiac Defibrillators: A Brief Report. Am J Gastroenterol 104(7): 1856-1857.

Ell C, Remke S, May A, Helou L, Henrich R, Mayer G (2002): The first prospective controlled trial comparing wireless capsule endoscopy with push enteroscopy in chronic gastrointestinal bleeding. Endoscopy 34(9): 685-689.

Fang YH, Chen CX, Zhang BL (2009): Effect of small bowel preparation with simethicone on capsule endoscopy. J Zhejiang Univ Sci B 10(1): 46-51.

Fireman Z, Paz D, Kopelman Y (2005): Capsule endoscopy: improving transit time and image view. World J Gastroenterol 11(37): 5863-5866.

Fischer D, Schreiber R, Levi D, Eliakim R (2004): Capsule endoscopy: the localization system. Gastrointest Endosc Clin N Am 14(1): 25-31.

Flohr T: Messprinzip, Bildrekonstruktion, Gerätetypen und Aufnahmetechniken - Einleitung; in: Wie funktioniert CT?; hrsg. v. Alkadhi H, Leschka S, Stolzman P, Scheffel H; 1. Auflage; Springer-Verlag, Berlin, Heidelberg 2011, S. 4.

Frieling T: Truncus coeliacus und Mesenterialgefäße; in: Gefässmedizin - Theorie und Praxis; hrsg. v. Cissarek T, Kröger K, Santosa F, Zeller T; 1. Auflage; ABW Wissenschaftsverlagsgesellschaft, Berlin 2009, S. 85-89.

Fritsch H, Kühnel W: Verdauungssystem: Dünndarm; in: Taschenatlas Anatomie - Band 2: Innere Organe; hrsg. v. Fritsch, H, Kühnel W; 10. Auflage; Georg Thieme Verlag KG, Stuttgart 2009, S. 196-201.

Fry LC, Carey EJ, Shiff AD, Heigh RI, Sharma VK, Post JK, Hentz JG, Fleischer DE, Leighton JA (2006): The yield of capsule endoscopy in patients with abdominal pain or diarrhea. Endoscopy 38(5): 498-502.

Ge ZZ, Chen HY, Gao YJ, Hu YB, Xiao SD (2006): The role of simeticone in small-bowel preparation for capsule endoscopy. Endoscopy $\underline{38}(8)$ : 836-840.

Given Imaging: PillCam Plattform - Bedienungsanleitung für RAPID 5.2; o. Hrsg.; Given Imaging Ltd., o. O. 2008.

Given Imaging (2013): PillCam SB3 Capsule- Feasibility Study. U. S. N. I. o. Health; Given Imaging Ltd.; in: http://clinicaltrials.gov/ct2/show/NCT01433042, 12/2013.

Gölder SK, Messmann H (2011): Akute gastrointestinale Blutungen. Der Gastroenterologe $\underline{6}(1)$.

Gölder SK, Schreyer AG, Endlicher E, Feuerbach S, Scholmerich J, Kullmann F, Seitz J, Rogler G, Herfarth H (2006): Comparison of capsule endoscopy and magnetic resonance (MR) enteroclysis in suspected small bowel disease. Int J Colorectal Dis 21(2): 97-104. 
Grützner G, Schmid-Tannwald C: Duale Reihe Radiologie; hrsg. v. Reiser M, Kuhn FP, Debus J; 3. Auflage; Georg Thieme Verlag KG, Stuttgart 2011.

Güldütuna S, Keuchel M: Praktischer Leitfaden der Kapselendoskopie; hrsg. v. Güldütuna S, Keuchel M; 1. Auflage; UNI-MED Verlag AG, Bremen 2009.

Hallas J, Dall M, Andries A, Andersen BS, Aalykke C, Hansen JM, Andersen M, Lassen AT (2006): Use of single and combined antithrombotic therapy and risk of serious upper gastrointestinal bleeding: population based case-control study. BMJ 333(7571): 726.

Hartmann D (2005): MRT in der Gastroenterologie. Medizinische Klinik 100(2): 115.

Hartmann D (2010): Dünndarmendoskopie. Der Internist 51(6): 711.

Hartmann D, Riemann JF (2009): Enteroskopische Techniken. Der Gastroenterologe 4(1): 9-18.

Hartmann D, Schmidt H, Bolz G, Schilling D, Kinzel F, Eickhoff A, Huschner W, Möller K, Jakobs R, Reitzig P, et al. (2005): A prospective two-center study comparing wireless capsule endoscopy with intraoperative enteroscopy in patients with obscure GI bleeding. Gastrointest Endosc 61(7): 826-832.

Hartmann D, Eickhoff A, Tamm R (2007): Balloon-assisted enteroscopy using a single-balloon technique. Endoscopy 39 Suppl 1: E276.

Hauser H (2006): Guidelines - malignant tumors of the small intestine. European Surgery $\underline{38}(2)$ : 118-123.

Henniges U, Rosien U: Mittlere gastrointestinale Blutung; in: Praktische Gastroenterologie; hrsg. v. Layer P, Rosien U; 4. Auflage; Elsevier GmbH, Urban \& Fischer Verlag, München 2011, S. 165.

Henniges U, Rosien U, Fibbe C, Pachmann C, Gaus A, Schönfeld JV, Payer P, Ell C: Enteroskopie; in: Praktische Gastroenterologie; hrsg. v. Layer P, Rosien U; 4. Auflage; Elsevier GmbH, Urban \& Fischer Verlag, München 2011, S. 55-57.

Henniges U, Rosien U, Fibbe C, Pachmann C, Gaus A, Schönfeld JV, Payer P, Ell C: Koloskopie; in: Praktische Gastroenterologie; hrsg. v. Layer P, Rosien U; 4. Auflage; Elsevier GmbH, Urban \& Fischer Verlag, München 2011, S. 53-54.

Henniges U, Rosien U, Layer P: Gastrointestinale Blutung; in: Praktische Gastroenterologie; hrsg. v. Layer P, Rosien U; 4. Auflage; Elsevier GmbH, Urban \& Fischer Verlag, München 2011, S. 18-19.

Henning H: Laparoskopie - Explorative Laparoskopie; in: Klinische Gastroenterologie - Band 1, hrsg. v. Hahn EG, Riemann JF; Georg Thieme Verlag KG, Stuttgart 2000, S. 198-203.

Herbay vA, Krekeler GR, Riede UN: Dünndarmkarzinom; in: Allgemeine und spezielle Pathologie; hrsg. v. Riede UN, Werner M, Schaefer HE; 5. Auflage; Georg Thieme Verlag KG, Stuttgart 2004, S. 708-709.

Herold G: Innere Medizin; hrsg. v. Herold G.; o. V., Köln 2012.

Heuss LT (2004): Kapselendoskopie. Medizin Spektrum - Gastroenterologie. Mediscope, Basel, 11/12.2004; in:

http://www.tellmed.ch/tellmed/Fachliteratur/Medizinspektrum/Kapselendoskopie.php.

Hölscher AH, Hölscher AC: Gefäßerkrankungen des Dünndarms; in: Chirurgie; hrsg. v. Siewert JR; Springer-Verlag, Berlin, Heidelberg 2006, S. 610-611.

Hünerbein R: Computertomografie (CT); in: Duale Reihe Radiologie; hrsg. v. Reiser M, Kuhn FP, Debus J; 3. Auflage; Georg Thieme Verlag KG, Stuttgart 2011, S. 73-79.

Iaquinto G, Fornasarig M, Quaia M, Giardullo N, D'Onofrio V, Iaquinto S, Di Bella S, Cannizzaro R (2008): Capsule endoscopy is useful and safe for small-bowel surveillance in familial adenomatous polyposis. Gastrointestinal endoscopy 67(1): 61-67.

Iddan G, Meron G, Glukhovsky A, Swain P (2000): Wireless capsule endoscopy. Nature 405(6785): 417. 
Iddan GJ, Swain CP (2004): History and development of capsule endoscopy. Gastrointest Endosc Clin N Am 14(1): 1-9.

Kantianis A, Karagiannis S, Liatsos C, Galanis P, Psilopoulos D, Tenta R, Kalantzis N, Mavrogiannis C (2009): Comparison of two schemes of small bowel preparation for capsule endoscopy with polyethylene glycol: a prospective, randomized single-blind study. Eur J Gastroenterol Hepatol 21(10): 1140-1144.

Keroack MD, Peralta R, Abramson SD, Misdraji J (2004): Case 24-2004: A 48-Year-Old Man with Recurrent Gastrointestinal Bleeding. N Engl J Med $\underline{351 .}$

Keuchel M, Hagenmüller F: Atlas der Videokapselendoskopie; hrsg. v. Keuchel M, Hagenmüller F; Springer-Verlag, Berlin, Heidelberg 2005.

Keuchel M, Gay G, Hagenmüller F: Vaskuläre Dünndarmerkrankungen - Arteriovenöse Erkrankungen; in: Atlas der Videokapselendoskopie; hrsg. v. Keuchel M, Hagenmüller F; Springer-Verlag, Berlin, Heidelberg 2005, S. 50-58.

Keuchel M, Steinbrück I, Hagenmüller F: Normaler Dünndarm; in: Atlas der Videokapselendoskopie; hrsg. v. Keuchel M, Hagenmüller F; Springer-Verlag, Berlin, Heidelberg 2005, S. 35-42.

Keuchel M, Thaler C, Hagenmüller F: Patency-Kapsel; in: Atlas der Videokapselendoskopie; hrsg. v. Keuchel M, Hagenmüller F; Springer-Verlag, Berlin, Heidelberg 2005, S. 153155.

Keuchel M, Selby WA, Tóth E: Benign Tumors; in: Atlas of Video Capsule Endoscopy; hrsg. v. Keuchel M, Hagenmüller F, Fleischer DE; Springer-Verlag, Berlin, Heidelberg 2006, S. 154-171.

Kirsch J: Dünndarm; in: Taschenlehrbuch Anatomie; hrsg. v. Kirsch J, May CA, Lorkeet D; Georg Thieme Verlag KG, Stuttgart 2011, S. 370-375.

Kohler B, Benz CA, Eickhoff A, Hartmann D, Jakobs R, Maier M, Riemann JF: Gastroenterologie; hrsg. v. Riemann JF, Fischbach W, Galle PR, Mössner J; Georg Thieme Verlag KG, Stuttgart 2010.

Koornstra JJ (2009): Bowel preparation before small bowel capsule endoscopy: what is the optimal approach? Eur J Gastroenterol Hepatol 21(10): 1107-1109.

Koulaouzidis A, Pendlebury J, Douglas S, Plevris JN (2009): Aspiration of video capsule: rare but potentially life-threatening complication to include in your consent form. Am $\mathbf{J}$ Gastroenterol 104(6): 1602-1603.

Ladas SD, Triantafyllou K, Spada C, Riccioni ME, Rey JF, Niv Y, Delvaux M, de Franchis R, Costamagna G, Committee ECG (2010): European Society of Gastrointestinal Endoscopy (ESGE): recommendations (2009) on clinical use of video capsule endoscopy to investigate small-bowel, esophageal and colonic diseases. Endoscopy 42(3): 220-227.

Lai LH, Wong GL, Chow DK, Lau JY, Sung JJ, Leung WK (2006): Long-term follow-up of patients with obscure gastrointestinal bleeding after negative capsule endoscopy. Am $\mathbf{J}$ Gastroenterol 101(6): 1224-1228.

Leschka S, Glaser-Gallion N: Leber, Milz, Pankreas, Nebennieren und Gastrointestinaltrakt Protokolle; in: Wie funktioniert CT?; hrsg. v. Alkadhi H, Leschka S, Stolzman P, Scheffel H; 1. Auflage; Springer-Verlag, Berlin, Heidelberg 2011, S. 90-100.

Leung WK, Chan FK, Fung SS, Wong MY, Sung JJ (2005): Effect of oral erythromycin on gastric and small bowel transit time of capsule endoscopy. World J Gastroenterol 11(31): 4865-4868.

Levinthal GN, Burke CA, Santisi JM (2003): The accuracy of an endoscopy nurse in interpreting capsule endoscopy. Am J Gastroenterol 98(12): 2669-2671. 
Lewis BS (1999): Medical and hormonal therapy in occult gastrointestinal bleeding. Semin Gastrointest Dis 10(2): 71-77.

Lewis BS (2000): Small intestinal bleeding. Gastroenterol Clin North Am 29(1): 67-95, vi.

Lewis BS, Swain P (2002): Capsule endoscopy in the evaluation of patients with suspected small intestinal bleeding: Results of a pilot study. Gastrointest Endosc 56(3): 349-353.

Lewis BS, Eisen GM, Friedman S (2005): A pooled analysis to evaluate results of capsule endoscopy trials. Endoscopy 37(10): 960-965.

Lewis BS, Keuchel M, Caselitz J: Malignant Tumors; in: Atlas of Video Capsule Endoscopy; hrsg. v. Keuchel M, Hagenmüller F, Fleischer D; 1. Auflage; Springer-Verlag, Berlin, Heidelberg 2006, S. 172-190.

Li F, Gurudu SR, De Petris G, Sharma VK, Shiff AD, Heigh RI, Fleischer DE, Post J, Erickson P, Leighton JA (2008): Retention of the capsule endoscope: a single-center experience of 1000 capsule endoscopy procedures. Gastrointest Endosc 68(1): 174-180.

Liao Z, Gao R, Xu C, Li ZS (2010): Indications and detection, completion, and retention rates of small-bowel capsule endoscopy: a systematic review. Gastrointest Endosc 71(2): 280286.

Litwiller D (2001): CCD vs. CMOS. Photonics Spectra 35(1): 154-158.

Lüllmann-Rauch R: Taschenlehrbuch Histologie; hrsg. v. Lüllmann-Rauch R; 3. Auflage; Georg Thieme Verlag KG, Stuttgart 2009.

Lupu A, Stefanescu C, Treton X, Attar A, Corcos O, Bouhnik Y (2013): Bevacizumab as rescue treatment for severe recurrent gastrointestinal bleeding in hereditary hemorrhagic telangiectasia. J Clin Gastroenterol 47(3): 256-257.

Marmo R, Rotondano G, Piscopo R, Bianco MA, Cipolletta L (2005): Meta-analysis: capsule enteroscopy vs. conventional modalities in diagnosis of small bowel diseases. Aliment Pharmacol Ther 22(7): 595-604.

Marmo R, Rotondano G, Casetti T, Manes G, Chilovi F, Sprujevnik T, Bianco MA, Brancaccio ML, Imbesi V, Benvenuti S, et al. (2009): Degree of concordance between doubleballoon enteroscopy and capsule endoscopy in obscure gastrointestinal bleeding: a multicenter study. Endoscopy 41(7): 587-592.

May A: Push-and-Pull-Enteroskopie in Doppel-Ballon-Technik; in: Interventionelle Endoskopie - Lehrbuch und Atlas; hrsg. v. Kahl S, Kähler G, Dormann A; 1. Auflage; Elsevier GmbH, Urban \& Fischer Verlag, München 2007, S. 198-204.

May A, Albert J, Keuchel M, Moog G, Hartmann D (2010): Capsule endoscopy for the diagnosis of small bowel diseases. An updated statement by the endoscopy section of DGVS. Z Gastroenterol 48(12): 1384-1404.

Meier PN, Manns MP: Angiodysplasien; in: Klinische Gastroenterologie und Stoffwechsel; hrsg. v. Adler G, Beglinger C, Manns MP, Müller-Lissner S, Schmiegel W; Springer-Verlag, Berlin, Heidelberg 2000, S. 983-986.

Melle U, Rosien U, Keller J, Layer P, Groß V: Morbus Crohn; in: Praktische Gastroenterologie; hrsg. v. Layer P, Rosien U; 4. Auflage; Elsevier GmbH, Urban \& Fischer Verlag, München 2011, S. 221-227.

Mensink PB, Haringsma J, Kucharzik T, Cellier C, Perez-Cuadrado E, Monkemuller K, Gasbarrini A, Kaffes AJ, Nakamura K, Yen HH, et al. (2007): Complications of double balloon enteroscopy: a multicenter survey. Endoscopy 39(7): 613-615.

Meron G: Development of the swallowable video capsule; in: Atlas of Capsule Endoscopy; hrsg. v. Halpern M, Jacob H; 1. Auflage; Given Imaging, Haifa (Israel) 2002, S. 3-5.

Metzger YC, Adler SN, Shitrit ABK, B. Bjarnason I (2009): Comparison of a new PillCam ${ }^{\mathrm{TM}}$ SB2 video capsule versus the standard PillCam ${ }^{\mathrm{TM}}$ SB for detection of small bowel disease. Dovepress Journal 2009:2: 7-11. 
Minardi AJ, Jr., Zibari GB, Aultman DF, McMillan RW, McDonald JC (1998): Small-bowel tumors. J Am Coll Surg 186(6): 664-668.

Mishkin D: Capsules; in: Capsule Endoscopy Simplified; hrsg. v. Franchis dR, Lewis B, Mishkin D; 1. Auflage; SLACK Incorporated, Thorofare (NJ, USA) 2010, S. 4-5.

Müller M: Dünndarmtumoren; in: Chirurgie - Für Studium und Praxis; hrsg. v. Müller M u. M; 11. Auflage; Medizinische Verlags- und Informationsdienste, Breisach a. Rh. 2012/13, S. 190-191.

Mylonaki M, Fritscher-Ravens A, Swain P (2003): Wireless capsule endoscopy: a comparison with push enteroscopy in patients with gastroscopy and colonoscopy negative gastrointestinal bleeding. Gut 52(8): 1122-1126.

Nakamura M, Niwa Y, Ohmiya N, Miyahara R, Ohashi A, Itoh A, Hirooka Y, Goto H (2006): Preliminary comparison of capsule endoscopy and double-balloon enteroscopy in patients with suspected small-bowel bleeding. Endoscopy 38(1): 59-66.

Nathan SR, Biernat L (2007): Aspiration - an important complication of small-bowel video capsule endoscopy. Endoscopy 39 Suppl 1: E343.

Niv E, Bonger I, Barkay O, Halpern Z, Mahajna E, Depsames R, Kopelman Y, Fireman Z (2008): Effect of erythromycin on image quality and transit time of capsule endoscopy: a two-center study. World J Gastroenterol 14(16): 2561-2565.

Niv Y, Niv G (2005): Capsule endoscopy examination--preliminary review by a nurse. Dig Dis Sci 50(11): 2121-2124.

Pachmann C, Rosien U, Schwarz W, Layer P, Gebel MJ: Ultraschallsonographie und Endosonographie; in: Praktische Gastroenterologie; hrsg. v. Layer P, Rosien U; 4. Auflage; Elsevier GmbH, Urban \& Fischer Verlag, München 2011, S. 62-71.

Park SC, Keum B, Seo YS, Kim YS, Jeen YT, Chun HJ, Um SH, Kim CD, Ryu HS (2011): Effect of bowel preparation with polyethylene glycol on quality of capsule endoscopy. Dig Dis Sci 56 (6): 1769-1775.

Pasha SF, Leighton JA, Das A, Harrison ME, Decker GA, Fleischer DE, Sharma VK (2008): Double-balloon enteroscopy and capsule endoscopy have comparable diagnostic yield in small-bowel disease: a meta-analysis. Clin Gastroenterol Hepatol 6(6): 671-676.

Pennazio M (2006): Capsule endoscopy: where are we after 6 years of clinical use? Dig Liver Dis $\underline{38}(12): 867-878$.

Pennazio M, Santucci R, Rondonotti E, Abbiati C, Beccari G, Rossini FP, De Franchis R (2004): Outcome of patients with obscure gastrointestinal bleeding after capsule endoscopy: report of 100 consecutive cases. Gastroenterology 126(3): 643-653.

Radeleff B, Stegen P, Kauffmann G: Magnetresonanztomografie (MRT); in: Radiologie; hrsg. v. Kauffmann G, Sauer R, Weber W; 4. Auflage; Elsevier GmbH, Urban \& Fischer Verlag, München 2011, S. 79-97.

Riede UN, Blum HE: Verdauungsorgane - Mitteldarm: Entzündungsmuster; in: Basiswissen Allgemeine und Spezielle Pathologie; hrsg. v. Riede UN, Werner M, Freudenberg N; Springer-Verlag, Berlin, Heidelberg 2009, S. 355-358.

Riemann JF, Rosenbaum A: Anamnese und körperliche Untersuchung; in: Gastroenterologie; hrsg. v. Riemann JF, Fischbach W, Galle PR, Mössner J; Georg Thieme Verlag KG, Stuttgart 2010, S. 142-147.

Rokkas T, Papaxoinis K, Triantafyllou K, Pistiolas D, Ladas SD (2009): Does purgative preparation influence the diagnostic yield of small bowel video capsule endoscopy?: A meta-analysis. Am J Gastroenterol 104(1): 219-227. 
Rondonotti E, Pennazio M, Toth E, Menchen P, Riccioni ME, De Palma GD, Scotto F, De Looze D, Pachofsky T, Tacheci I, et al. (2008): Small-bowel neoplasms in patients undergoing video capsule endoscopy: a multicenter European study. Endoscopy 40(6): 488-495.

Rondonotti E, Soncini M, Girelli C, Ballardini G, Bianchi G, Brunati S, Centenara L, Cesari P, Cortelezzi C, Curioni S, et al. (2010): Small bowel capsule endoscopy in clinical practice: a multicenter 7-year survey. Eur J Gastroenterol Hepatol 22(11): 1380-1386.

Rosien U, Melle U, Layer P, Keller J, Groß V: Colitis ulcerosa; in: Praktische Gastroenterologie; hrsg. v. Layer P, Rosien U; 4. Auflage; Elsevier GmbH, Urban \& Fischer Verlag, München 2011, S. 214-220.

Rosien U, Panter H, Andresen V, Layer P: Diarrhoe; in: Praktische Gastroenterologie; hrsg. v. Layer P, Rosien U; 4. Auflage; Elsevier GmbH, Urban \& Fischer Verlag, München 2011, S. 20-23.

Sami SS, Al-Araji SA, Ragunath K (2014): Review article: gastrointestinal angiodysplasia pathogenesis, diagnosis and management. Aliment Pharmacol Ther 39(1): 15-34.

Saperas E, Dot J, Videla S, Alvarez-Castells A, Perez-Lafuente M, Armengol JR, Malagelada JR (2007): Capsule endoscopy versus computed tomographic or standard angiography for the diagnosis of obscure gastrointestinal bleeding. Am J Gastroenterol 102(4): 731-737.

Sauerbruch T (2008): Effiziente Diagnostik und Therapie oberer gastrointestinaler Blutungen: Schlusswort. Dtsch Arztebl International 105(18): 346.

Saurin JC, Delvaux M, Gaudin JL, Fassler I, Villarejo J, Vahedi K, Bitoun A, Canard JM, Souquet JC, Ponchon T, et al. (2003): Diagnostic value of endoscopic capsule in patients with obscure digestive bleeding: blinded comparison with video push-enteroscopy. Endoscopy 35(7): 576-584.

Scharinger L, Aigner E, Datz C (2007): Diagnostik der obskuren gastrointestinalen Blutung Stellenwert von Kapselendoskopie und Doppelballonenteroskopie. Journal für Gastroenterologische und Hepatologische Erkrankungen 5 (4): 22-27.

Schlag C, Neu B, Menzel C, Nennstiel S, Phillip V, Schmid RM Delius Sv (2013): "NotfallVideo-Kapsel-Endoskopie bei akuter GI-Blutung (NOKA-Studie)." Z Gastroenterol 51(09): PP2.

Schünke M, Schulte E, Schumacher U: Prometheus - LernAtlas der Anatomie - Hals und Innere Organe; hrsg. v. Schünke M, Schulte E, Schumacher U; 1. Auflage; Georg Thieme Verlag KG, Stuttgart 2005.

Selby W (2005): Complete small-bowel transit in patients undergoing capsule endoscopy: determining factors and improvement with metoclopramide. Gastrointest Endosc 61(1): $80-85$.

Steinbrück I, Keuchel M, Hagenmüller F (2010): Technische Neuerungen in der Videokapselendoskopie. Endo heute 23(02): 113,117.

Stich MIJ, Borisov SM, Henne U, Schäferling M (2009): Read-out of multiple optical chemical sensors by means of digital color cameras. Sensors and Actuators B: Chemical 139(1): 204-207.

Südhoff T, Schmiegel W: Tumoren des Dünndarms und gastroenteropankreatische neuroendokrine Tumoren; in: Medizinische Therapie 2007 | 2008; hrsg. v. Schölmerich J; Springer-Verlag, Berlin, Heidelberg 2007, S. 829-836.

Sugano K, Marcon N (2007): The First International Workshop on Double Balloon Endoscopy: a consensus meeting report. Gastrointest Endosc 66 (3 Suppl): 7-11.

Thiel S, Jesse N, Rosien U: Einheimische Sprue (Zöliakie); in: Praktische Gastroenterlogie; hrsg. v. Layer P, Rosien U; 4. Auflage; Elsevier GmbH, Urban \& Fischer Verlag, München 2011, S. 149-151. 
Timmer A, Goebell H (1999): Incidence of ulcerative colitis, 1980-1995 - a prospective study in an urban population in Germany. Z Gastroenterol 37(11): 1079-1084.

Toy E, Rojany M, Sheikh R, Mann S, Prindiville T (2008): Capsule Endoscopy's Impact on Clinical Management and Outcomes: A Single-Center Experience With 145 Patients. Am J Gastroenterol 103(12): 3022-3028.

Triantafyllou K, Kalantzis C, Papadopoulos AA, Apostolopoulos P, Rokkas T, Kalantzis N, Ladas SD (2007): Video-capsule endoscopy gastric and small bowel transit time and completeness of the examination in patients with diabetes mellitus. Dig Liver Dis $\underline{39}$ (6): 575-580.

Triantafyllou K, Kalli T, Ladas SD (2008): Small bowel purge after the entrance of the capsule in the duodenum results to better quality of bowel preparation for video-capsule endoscopy. Prospective, randomized, double-blind placebo-controlled, real time viewer assisted study. Endoscopy 40 (Suppl I): A30-30.

Triester SL, Leighton JA, Leontiadis GI, Fleischer DE, Hara AK, Heigh RI, Shiff AD, Sharma VK (2005): A meta-analysis of the yield of capsule endoscopy compared to other diagnostic modalities in patients with obscure gastrointestinal bleeding. Am J Gastroenterol 100(11): 2407-2418.

Velayos Jimenez B, Fernandez Salazar L, Aller de la Fuente R, de la Calle Valverde F, Del Olmo Martinez L, Arranz Santos T, Gonzalez Hernandez J (2005): Study of gastrointestinal transit times with capsule endoscopy. Gastroenterol Hepatol 28 (6): 315320.

Voderholzer W, Kornbluth A, Legmani PE, Leigton JA: Crohn's Disease; in: Atlas of Video Capsule Endoscopy; hrsg. v. Keuchel M, Hagenmüller F, Fleischer DE; 1. Auflage; Springer-Verlag, Berlin, Heidelberg 2006, S. 108-116.

Voderholzer WA, Ortner M, Rogalla P, Beinholzl J, Lochs H (2003): Diagnostic yield of wireless capsule enteroscopy in comparison with computed tomography enteroclysis. Endoscopy 35(12): 1009-1014.

Voderholzer WA, Beinhoelzl J, Rogalla P, Murrer S, Schachschal G, Lochs H, Ortner MA (2005): Small bowel involvement in Crohn's disease: a prospective comparison of wireless capsule endoscopy and computed tomography enteroclysis. Gut 54(3): 369-373.

Washington K, McDonagh D (1995): Secondary tumors of the gastrointestinal tract: surgical pathologic findings and comparison with autopsy survey. Mod Pathol $\underline{8}(4)$ : 427-433.

Wei W, Ge ZZ, Lu H, Gao YJ, Hu YB, Xiao SD (2008): Purgative bowel cleansing combined with simethicone improves capsule endoscopy imaging. Am J Gastroenterol 103(1): 7782.

Wismer E: Orales Kontrastmittel; in: Wie funktioniert CT?; hrsg. v. Alkadhi H, Leschka S, Stolzmann P, Scheffel H; 1. Auflage; Springer-Verlag, Berlin, Heidelberg 2011, S. 48.

Zuckerman GR, Prakash C, Askin MP, Lewis BS (2000): AGA technical review on the evaluation and management of occult and obscure gastrointestinal bleeding. Gastroenterology 118(1): 201-221. 